NIST Special Publication 1086r1 December 2012 Revision

\title{
CFAST - Consolidated Model of Fire Growth and Smoke Transport (Version 6) Software Development and Model Evaluation Guide
}

Richard D. Peacock

Paul A. Reneke

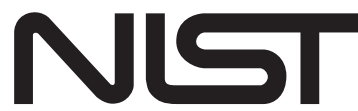

National Institute of Standards and Technology 

NIST Special Publication 1086r1

December 2012 Revision

\section{CFAST - Consolidated Model of Fire Growth and Smoke Transport (Version 6) Software Development and Model Evaluation Guide}

Richard D. Peacock

Paul A. Reneke

Fire Research Division

Engineering Laboratory

http://dx.doi.org/10.6028/NIST.SP.1086r1

March 2013

SVNRepository Revision : 507

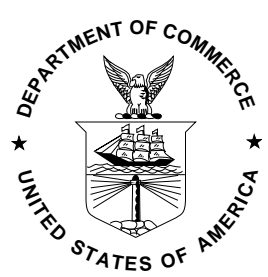

U.S. Department of Commerce Rebecca Blank, Acting Secretary

National Institute of Standards and Technology Patrick D. Gallagher, Under Secretary of Commerce for Standards and Technology and Director 



\section{Disclaimer}

The U. S. Department of Commerce makes no warranty, expressed or implied, to users of CFAST and associated computer programs, and accepts no responsibility for its use. Users of CFAST assume sole responsibility under Federal law for determining the appropriateness of its use in any particular application; for any conclusions drawn from the results of its use; and for any actions taken or not taken as a result of analyses performed using these tools. CFAST is intended for use only by those competent in the field of fire safety and is intended only to supplement the informed judgment of a qualified user. The software package is a computer model which may or may not have predictive value when applied to a specific set of factual circumstances. Lack of accurate predictions by the model could lead to erroneous conclusions with regard to fire safety. All results should be evaluated by an informed user. 


\section{Intent and Use}

The algorithms, procedures, and computer programs described in this report constitute a methodology for predicting some of the consequences resulting from a prescribed fire. They have been compiled from the best knowledge and understanding currently available, but have important limitations that must be understood and considered by the user. The program is intended for use by persons competent in the field of fire safety and with some familiarity with personal computers. It is intended as an aid in the fire safety decision-making process. 


\section{Preface}

This supplement to the CFAST Technical Reference Guide provides details of the software development process for CFAST and accompanying experimental evaluation of the model. It is based in part on the "Standard Guide for Evaluating the Predictive Capability of Deterministic Fire Models," ASTM E 1355 [?]. ASTM E 1355 defines model evaluation as "the process of quantifying the accuracy of chosen results from a model when applied for a specific use." The model evaluation process consists of two main components: verification and validation. Verification is a process to check the correctness of the solution of the governing equations. Verification does not imply that the governing equations are appropriate; only that the equations are being solved correctly. Validation is a process to determine the appropriateness of the governing equations as a mathematical model of the physical phenomena of interest. Typically, validation involves comparing model results with experimental measurement. Differences that cannot be explained in terms of numerical errors in the model or uncertainty in the measurements are attributed to the assumptions and simplifications of the physical model.

Evaluation is critical to establishing both the acceptable uses and limitations of a model. Throughout its development, CFAST has undergone various forms of evaluation, both at the National Institute of Standards and Technology and beyond. This Supplement provides a survey of validation work conducted to date to evaluate CFAST. Documentation of CFAST Verification is contained in the CFAST Technical Reference Guide [?]. 


\section{Acknowledgments}

Continuing support for CFAST is via internal funding at the National Institute of Standards and Technology (NIST). In addition, support is provided by other agencies of the U.S. Federal Government, most notably the Nuclear Regulatory Commission (NRC) Office of Research and the U.S. Department of Energy. The U.S. NRC Office of Research has funded key validation experiments, the preparation of the CFAST manuals, and the continuing development of sub-models that are of importance in the area of nuclear power plant safety. Special thanks to Mark Salley and Jason Dreisbach for their efforts and support. Support to refine the software development and quality assurance process for CFAST has been provided by the U.S. Department of Energy (DOE). The assistance of Subir Sen and Debra Sparkman in understanding DOE software quality assurance programs and the application of the process to CFAST is gratefully acknowledged. Thanks are also due to Allan Coutts, Washington Safety Management Solutions for his insight into the application of fire models to nuclear safety applications and detailed review of the CFAST document updates for DOE.

For the NIST/NRC tests, Alex Maranghides was in charge of the Large Fire Laboratory at NIST where these tests were conducted, and helped to design the experiments. Thanks also to technicians Laurean Delauter, Jay McElroy, and Jack Lee who constructed and conducted these validation experiments at NIST. Steve Nowlen at Sandia National Laboratories provided data, documentation and further information for the NRC-sponsored experiments referred to in this document and the "FM/SNL Test Series" (Factory Mutual and Sandia National Laboratories conducted these experiments). Finally, thanks to VTT, Finland, for their contribution of experimental data, referred to in this document as the "VTT Large Hall Experiments." 


\section{Contents}

Disclaimer

Intent and Use iii

$\begin{array}{ll}\text { Preface } & \text { v }\end{array}$

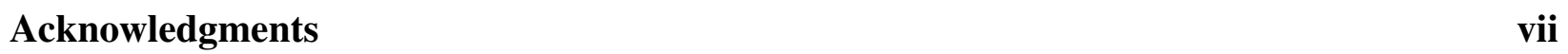

$\begin{array}{lll}1 & \text { Overview } & 1\end{array}$

$1.1 \quad$ Software Development and Quality Assurance . . . . . . . . . . . . . . . . . . 2

1.2 Model Validation Scenarios . . . . . . . . . . . . . . . . . . . . . . . 2

1.3 Input Data Required to Run the Model . . . . . . . . . . . . . . . . . . 3

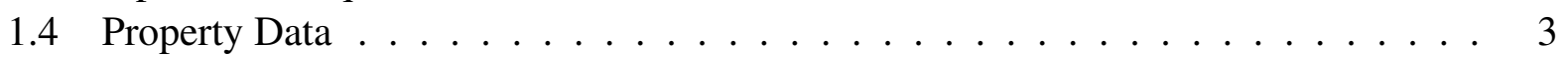

1.5 Model Outputs . . . . . . . . . . . . . . . . . . . . 4

1.6 Model Accuracy . . . . . . . . . . . . . . . . . . . . . . . . . . . 4

1.7 Uses and Limitations of the Model . . . . . . . . . . . . . . . . . . . . . . . 5

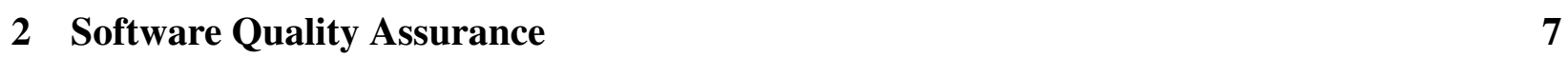

$2.1 \quad$ Relevant Publications $\ldots \ldots \ldots \ldots \ldots$. . . . . . . . . . . . . . 7

2.2 Model Management $\ldots \ldots \ldots \ldots$. . . . . . . . . . . . . . . . . . . . . . . . . . . . . . . .

2.3 SQA Documentation $\ldots \ldots \ldots \ldots \ldots$

2.4 Standards, Practices, Conventions, and Metrics $\ldots \ldots \ldots \ldots$

2.5 Software Reviews . . . . . . . . . . . . . . . . . . . . . . . . . . . . . . . . . . . . .

2.6 Model Testing . . . . . . . . . . . . . . . . . . . . . 10

2.7 Problem Reporting and Resolution . . . . . . . . . . . . . . . . . . . . . . . . . . . . . . .

2.8 Tools, Techniques, and Methodologies . . . . . . . . . . . . . . . . . . . . . . . . . . . . . . . .

2.9 Media Control . . . . . . . . . . . . . . . . . . . . . . . . 11

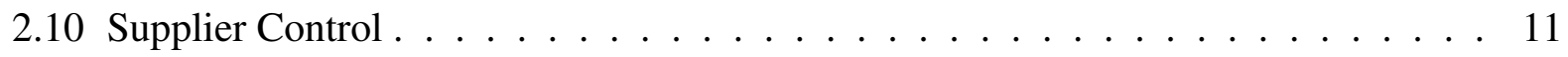

2.11 Records Collection, Maintenance, and Retention . . . . . . . . . . . . . . . . . 12

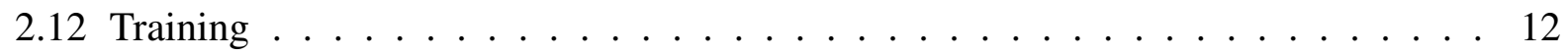

2.13 Risk Management . . . . . . . . . . . . . . . . . . . . . . . 12

3 Software Structure and Robustness

3.1 Structure of the Numerical Routines . . . . . . . . . . . . . . . . . . . . . . . . . . . .

3.2 Comparison with Analytic Solutions . . . . . . . . . . . . . . . 15 
3.3 Code Checking . . . . . . . . . . . . . . . . . . . . . . 15

3.4 Numerical Tests $\ldots \ldots \ldots \ldots \ldots \ldots$

\begin{tabular}{|lrl}
4 & Survey of Past Validation Work & 17
\end{tabular}

4.1 Comparisons with Full-Scale Tests Conducted Specifically for the Chosen Evaluation 17

4.2 Comparisons with Previously Published Test Data . . . . . . . . . . . . . . . . . . 19

$4.2 .1 \quad$ Fire Plumes . . . . . . . . . . . . . . . . . . . . . . . . . . . . . . . . 19

4.2 .2 Multiple Compartments . . . . . . . . . . . . . . . 20

$4.2 .3 \quad$ Large Compartments . . . . . . . . . . . . . . . . . . . . 20

4.2 .4 Prediction of Flashover . . . . . . . . . . . . . . . . . . . . 20

4.3 Comparison with Documented Fire Experience $\ldots \ldots$. . . . . . . . . . 23

4.4 Comparison with Experiments Which Cover Special Situations . . . . . . . . . . . 24

$4.4 .1 \quad$ Nuclear Facilities . . . . . . . . . . . . . . . . . . . . . . . . . . . 24

4.4 .2 Small Scale Testing . . . . . . . . . . . . . . . . . . . . . . 25

4.4 .3 Unusual Geometry and Specific Algorithms . . . . . . . . . . . . . . 25

5 Description of Experiments 29

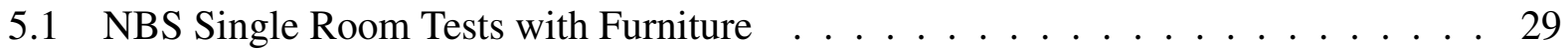

5.2 VTT Large Hall Tests . . . . . . . . . . . . . . . . . . . . . . . . . . 30

5.3 NIST/NRC Test Series $\ldots \ldots \ldots$. . . . . . . . . . . . . . . . . . . . . . . . . . . . 34

$5.4 \quad$ FM/SNL Test Series $\ldots \ldots \ldots$

5.5 iBMB Compartment Tests . . . . . . . . . . . . . . . . . . 40

5.6 NBS Multi-Compartment Test Series . . . . . . . . . . . . . . . . . . . . . . 43

$5.7 \quad$ FM Four Room Including Corridor Test Series . . . . . . . . . . . . . . . . . . . . 49

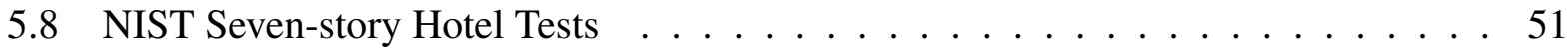

5.9 NIST / Navy High Bay Hanger Tests $\ldots \ldots \ldots \ldots$

$6 \quad$ Hot Gas Layer Temperature and Depth $\quad 53$

6.1 Model / Experiment Comparisons . . . . . . . . . . . . . . . . . 55

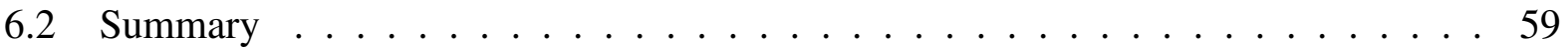

\begin{tabular}{|lll}
7 & Flame Height, Plume Temperature and Ceiling Jets & 61
\end{tabular}

7.1 Flame Height $\ldots \ldots \ldots \ldots$. . . . . . . . . . . . . . . . . . . 61

7.2 Plume Temperature . . . . . . . . . . . . . . . . . . . . . . 61

7.3 Ceiling Jets $\ldots \ldots \ldots \ldots$. . . . . . . . . . . . . . . . . 63

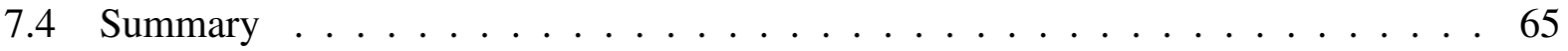

\begin{tabular}{lll}
\hline Gas Species and Smoke & 67
\end{tabular}

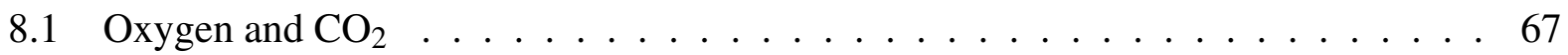

8.2 NIST/NRC Test Series, Smoke $\ldots \ldots \ldots$. . . . . . . . . . . . . 70

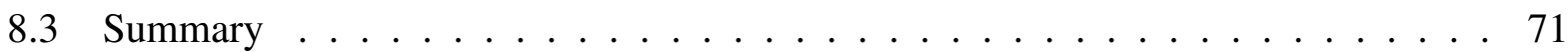

\begin{tabular}{lll}
\hline 9 & Pressure & 73
\end{tabular} 


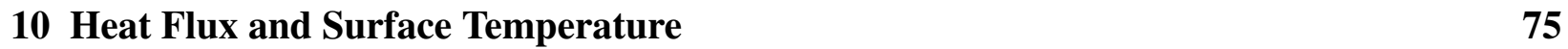

10.1 NIST/NRC Test Series, Cables $\ldots \ldots \ldots$. . . . . . . . . . . . . . 75

10.2 NIST/NRC Test Series, Compartment Walls, Floor and Ceiling . . . . . . . . . . 76

10.3 Summary . . . . . . . . . . . . . . . . . . 77

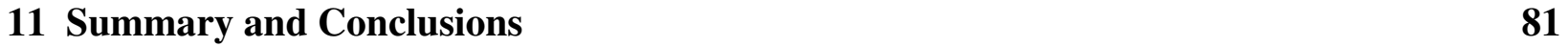

\begin{tabular}{ll}
\hline References & 85
\end{tabular}

\begin{tabular}{ll}
\hline Appendices & 85
\end{tabular}

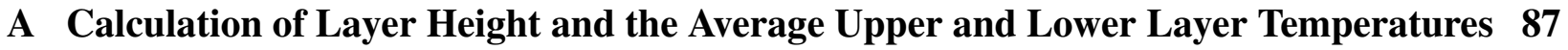

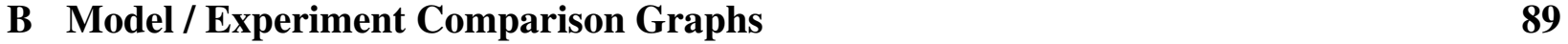

B.1 NBS Single Room Tests with Furniture . . . . . . . . . . . . . . . . . . 89

B.2 VTT Large Hall Tests . . . . . . . . . . . . . . . . . . . . . . . . . . . . . . . . . . . . . . . . . . . . . . . . . . .

B.3 NIST/NRC Test Series . . . . . . . . . . . . . . . . . . . . . 95

B.4 FM/SNL Test Series . . . . . . . . . . . . . . . . . . . . . . . . 141

B.5 iBMB Compartment Tests . . . . . . . . . . . . . . . . . . . . . . . . . . . . . . . . . . . . . . . . .

B.6 NBS Multi-Compartment Test Series . . . . . . . . . . . . . . . . . . . . . . . 155

B.7 $\quad$ FM Four Room Including Corridor Test Series . . . . . . . . . . . . . . . . . . . 159

B.8 NIST Seven-story Hotel Tests . . . . . . . . . . . . . . . . . . . 164 


\section{List of Figures}

$2.1 \quad$ CFAST SQA Organization Structure. . . . . . . . . . . . . . . . . . 8

3.1 Subroutine structure for the CFAST model . . . . . . . . . . . . . . . . . . . 14

4.1 Comparison of correlations, CFAST predictions, and experimental data for the prediction of flashover in a compartment fire. . . . . . . . . . . . 22

5.1 Plan and elevation view schematic of experimental room for NBS single room tests with furniture . . . . . . . . . . . . . . . . . . 30

5.2 Cut-Away View of Case 2 of the VTT Large Hall Tests. . . . . . . . . . . . . . . 31

5.3 Plan, side and perspective schematic drawings of the experimental arrangement of the VTT large hall fire tests, including the fuel pan $\ldots \ldots \ldots 32$

5.4 Photo of a $2 \mathrm{MW}$ heptane fire during the VTT large hall tests. Photo provided by Simo Hostikka, VTT. . . . . . . . . . . . . . . . . 33

$5.5 \quad$ Prescribed Heat Release Rate as a Function of Time for VTT Large Hall Tests. . 35

5.6 Photograph of a $1 \mathrm{MW}$ heptane fire seen through the open doorway. Photo provided by Anthony Hamins, NIST. . . . . . . . . . . . . . . . 36

$5.7 \quad$ Cross-section View of the NIST NRC Test Configuration. . . . . . . . . . . . . . . 36

5.8 Plan, side and perspective schematic drawings of the NIST NRC experimental $\begin{array}{ll}\text { arrangement. The fuel pan and cables B, D, F, and G (dotted lines) are also shown. } & 37\end{array}$

5.9 Measured and prescribed heat release rate as a function of time during Test 3 of the NIST NRC test series . . . . . . . . . . . . . . . . . . . . . 39

5.10 Detailed plan, side, and perspective schematic drawings of the FM/SNL experimental arrangement, including the supply and exhaust ducts, and the fuel pan. . . . 41

5.11 Prescribed (dotted line) and measured (solid line) heat release rate as a function of time during Test 21 of the FM/SNL test series . . . . . . . . . . . . . . . . 43

5.12 Detailed plan, side, and perspective schematic drawings of the iBMB pool fire experimental arrangement. . . . . . . . . . . . . . . . 44

5.13 Estimated heat release rate for the iBMB fire experiments. . . . . . . . . . . 45

5.14 Detailed plan, side, and perspective schematic drawings of the iBMB cable fire experimental arrangement. . . . . . . . . . . . . . . 46

5.15 Photo of a $100 \mathrm{~kW}$ fire with the burner located against the rear wall of one of the small compartments in the NBS Multi-Compartment test Series. . . . . . . . . . . 47

5.16 Overview of the NBS Test Configuration. . . . . . . . . . . . . . . 47

5.17 Plan, side and perspective schematic drawings of the NBS experimental arrangement, including the burner. . . . . . . . . . . . . . . . 48 
5.18 Prescribed and measured heat release rate as a function of time during Tests 100A and 100Z of the NBS multi-room test series. . . . . . . . . . . . . . . . 49

5.19 Overview of the Factory Mutual Four Room test series. . . . . . . . . . . . . . . 50

5.20 Overview of the NIST Seven-story hotel test series including smoke control. . . . . 52

6.1 Comparison of Measured and Predicted HGL Temperature and Height. . . . . . . . 55

6.2 Measured and Predicted HGL Temperature and Height for a Single Compartment

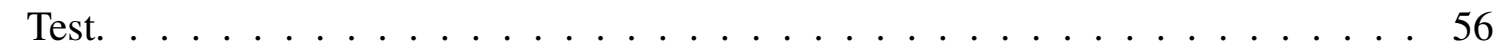

7.1 Comparison of Measured and Predicted Plume Centerline Temperature. . . . . . . 62

$7.2 \quad$ Comparison of Measured and Predicted Ceiling Jet Temperature. . . . . . . . . . . 64

8.1 Comparison of Measured and Predicted Oxygen Concentration and Carbon Dioxide Concentration. . . . . . . . . . . . . . . . 68

8.2 Comparison of Measured and Predicted Smoke Concentration. . . . . . . . . . . . 70

9.1 Comparison of Measured and Predicted Compartment Pressure. . . . . . . . . . . 74

10.1 Comparisons of Measured and Predicted Heat Flux to Targets and Target Temperature 78

10.2 Comparisons of Measured and Predicted Heat Flux to Compartment Surfaces and Surface Temperature . . . . . . . . . . . . . . 79

B.1 Predicted HGL Temperature and Height for the NBS Single Compartment Tests. . 90

B.2 $\quad$ Predicted Oxygen Concentrationfor the NBS Single Compartment Tests. . . . . . . 91

B.3 Predicted Compartment Pressure for the NBS Single Compartment Tests. . . . . . 91

B.4 $\quad$ Predicted HGL Temperature and Height for the VTT Large Hall Tests. . . . . . . . 93

B.5 $\quad$ Predicted Plume Centerline Temperature for the VTT Large Hall Tests.. . . . . . . 94

B.6 $\quad$ Predicted HGL Temperature and Height for the NIST/NRC Tests 1, 7, 2 and 8. . . 96

B.7 Predicted HGL Temperature and Height for the NIST/NRC Tests 4, 10, 13 and 16.97

B.8 $\quad$ Predicted HGL Temperature and Height for the NIST/NRC Tests 17, 3 and 9. . . . 98

B.9 Predicted HGL Temperature and Height for the NIST/NRC Tests 5, 14, 15 and 18.99

B.10 Ceiling Jet Temperature for the NIST/NRC Series, Closed Door Tests. . . . . . . . 100

B.11 Ceiling Jet Temperature for the NIST/NRC Series, Open Door Tests. . . . . . . . . 101

B.12 Predicted Oxygen and Carbon Dioxide for the NIST/NRC Tests 1, 7, 2 and 8. . . . 102

B.13 Predicted Oxygen and Carbon Dioxide for the NIST/NRC Tests 4, 10, 13 and 16.

B.14 Predicted Oxygen and Carbon Dioxide for the NIST/NRC Tests 17, 3, and 9. . . . 104

B.15 Predicted Oxygen and Carbon Dioxide for the NIST/NRC Tests 5, 14, 15 and 18. . 105

B.16 Smoke Concentrationfor the NIST/NRC Series, Closed Door Tests. . . . . . . . . 106

B.17 Smoke Concentrationfor the NIST/NRC Series, Open Door Tests. . . . . . . . . 107

B.18 Compartment Pressures for the NIST/NRC Series, Closed Door Tests. . . . . . . . 108

B.19 Compartment Pressures for the NIST/NRC Series, Open Door Tests. . . . . . . . . 109

B.20 NIST/NRC Series, Cable B Temperature and Heat Flux, Replicate Tests 1 and $7 . \quad$. 110

B.21 NIST/NRC Series, Cable B Temperature and Heat Flux, Replicate Tests 2 and 8.

B.22 NIST/NRC Series, Cable B Temperature and Heat Flux, Replicate Tests 4 and 10. . 111

B.23 NIST/NRC Series, Cable B Temperature and Heat Flux, Replicate Tests 13 and 16.111

B.24 NIST/NRC Series, Cable B Temperature and Heat Flux, Replicate Tests 3 and 9. 
B.25 NIST/NRC Series, Cable B Temperature and Heat Flux, Replicate Tests 5 and 14. . 112 B.26 NIST/NRC Series, Cable B Temperature and Heat Flux, Replicate Tests 15 and 18.113 B.27 NIST/NRC Series, Cable D Temperature and Heat Flux, Replicate Tests 1 and 7. . 114

B.28 NIST/NRC Series, Cable D Temperature and Heat Flux, Replicate Tests 2 and 8. B.29 NIST/NRC Series, Cable D Temperature and Heat Flux, Replicate Tests 4 and 10. 115

B.30 NIST/NRC Series, Cable D Temperature and Heat Flux, Replicate Tests 13 and 16. 115

B.31 NIST/NRC Series, Cable D Temperature and Heat Flux, Replicate Tests 3 and 9.116

B.32 NIST/NRC Series, Cable D Temperature and Heat Flux, Replicate Tests 5 and 14. 116

B.33 NIST/NRC Series, Cable D Temperature and Heat Flux, Replicate Tests 15 and 18.117

B.34 NIST/NRC Series, Cable F Temperature and Heat Flux, Replicate Tests 1 and 7. . . 118

B.35 NIST/NRC Series, Cable F Temperature and Heat Flux, Replicate Tests 2 and 8. . . 118

B.36 NIST/NRC Series, Cable F Temperature and Heat Flux, Replicate Tests 4 and 10. . 119

B.37 NIST/NRC Series, Cable F Temperature and Heat Flux, Replicate Tests 13 and 16.119

B.38 NIST/NRC Series, Cable F Temperature and Heat Flux, Replicate Tests 3 and 9. . . 120

B.39 NIST/NRC Series, Cable F Temperature and Heat Flux, Replicate Tests 5 and 14. . 120

B.40 NIST/NRC Series, Cable F Temperature and Heat Flux, Replicate Tests 15 and 18.121

B.41 NIST/NRC Series, Cable G Temperature and Heat Flux, Replicate Tests 1 and 7.

B.42 NIST/NRC Series, Cable G Temperature and Heat Flux, Replicate Tests 2 and 8.122

B.43 NIST/NRC Series, Cable G Temperature and Heat Flux, Replicate Tests 4 and 10. . 123

B.44 NIST/NRC Series, Cable G Temperature and Heat Flux, Replicate Tests 13 and 16. 123

B.45 NIST/NRC Series, Cable G Temperature and Heat Flux, Replicate Tests 3 and 9.

B.46 NIST/NRC Series, Cable G Temperature and Heat Flux, Replicate Tests 5 and 14. 124

B.47 NIST/NRC Series, Cable G Temperature and Heat Flux, Replicate Tests 15 and 18.125

B.48 NIST/NRC Series, Long Wall Temperature and Heat Flux, Replicate Tests 1 and 7.126

B.49 NIST/NRC Series, Long Wall Temperature and Heat Flux, Replicate Tests 2 and 8.126

B.50 NIST/NRC Series, Long Wall Temperature and Heat Flux, Replicate Tests 4 and 10. 127

B.51 NIST/NRC Series, Long Wall Temperature and Heat Flux, Replicate Tests 13 and

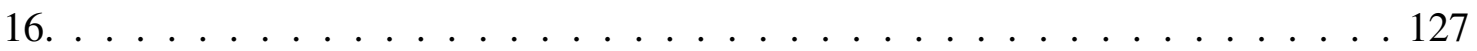

B.52 NIST/NRC Series, Long Wall Temperature and Heat Flux, Replicate Tests 3 and 9. 128

B.53 NIST/NRC Series, Long Wall Temperature and Heat Flux, Replicate Tests 5 and 14. 128

B.54 NIST/NRC Series, Long Wall Temperature and Heat Flux, Replicate Tests 15 and

18. . . . . . . . . . . . . . . . . . . . 129

B.55 NIST/NRC Series, Short Wall Temperature and Heat Flux, Replicate Tests 1 and 7.130

B.56 NIST/NRC Series, Short Wall Temperature and Heat Flux, Replicate Tests 2 and 8.130

B.57 NIST/NRC Series, Short Wall Temperature and Heat Flux, Replicate Tests 4 and 10.131

B.58 NIST/NRC Series, Short Wall Temperature and Heat Flux, Replicate Tests 13 and

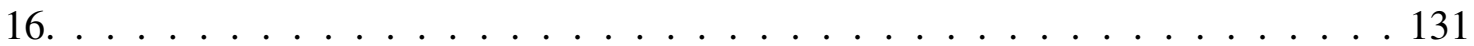

B.59 NIST/NRC Series, Short Wall Temperature and Heat Flux, Replicate Tests 3 and 9.132

B.60 NIST/NRC Series, Short Wall Temperature and Heat Flux, Replicate Tests 5 and 14. 132

B.61 NIST/NRC Series, Short Wall Temperature and Heat Flux, Replicate Tests 15 and

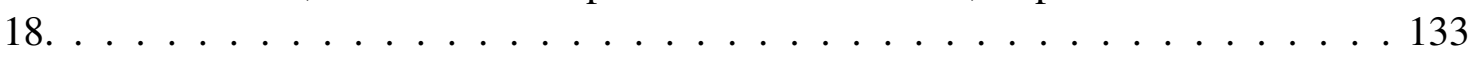

B.62 NIST/NRC Series, Ceiling Temperature and Heat Flux, Replicate Tests 4 and 10. . 134

B.63 NIST/NRC Series, Ceiling Temperature and Heat Flux, Replicate Tests 13 and 16. 134

B.64 NIST/NRC Series, Ceiling Temperature and Heat Flux, Replicate Tests 3 and 9. . . 135

B.65 NIST/NRC Series, Ceiling Temperature and Heat Flux, Replicate Tests 5 and 14. 
B.66 NIST/NRC Series, Ceiling Temperature and Heat Flux, Replicate Tests 15 and 18. 136 B.67 NIST/NRC Series, Floor Temperature and Heat Flux, Replicate Tests 1 and 7. . . . 137 B.68 NIST/NRC Series, Floor Temperature and Heat Flux, Replicate Tests 2 and 8. . . . 137 B.69 NIST/NRC Series, Floor Temperature and Heat Flux, Replicate Tests 4 and 10. . . 138 B.70 NIST/NRC Series, Floor Temperature and Heat Flux, Replicate Tests 13 and 16. . . 138 B.71 NIST/NRC Series, Floor Temperature and Heat Flux, Replicate Tests 3 and 9. . . . 139 B.72 NIST/NRC Series, Floor Temperature and Heat Flux, Replicate Tests 5 and 14. . . 139 B.73 NIST/NRC Series, Floor Temperature and Heat Flux, Replicate Tests 15 and 18. . . 140 B.74 Hot Gas Layer Temperature and Height for the FM/SNL Tests. . . . . . . . . . . . 142 B.75 Hot Gas Layer Temperature and Height for the FM/SNL Tests. . . . . . . . . . . . 143 B.76 Hot Gas Layer Temperature and Height for the FM/SNL Tests. . . . . . . . . . . . 144 B.77 Hot Gas Layer Temperature and Height for the FM/SNL Tests. . . . . . . . . . . . 145 B.78 Hot Gas Layer Temperature and Height for the FM/SNL Tests. . . . . . . . . . . . 146 B.79 Predicted Plume Centerline Temperature for the FM/SNL Tests. . . . . . . . . . 147 B.80 Predicted Plume Centerline Temperature for the FM/SNL Tests. . . . . . . . . . . 148 B.81 Predicted Plume Centerline Temperature for the FM/SNL Tests. . . . . . . . . . . 149 B.82 Predicted Plume Centerline Temperature for the FM/SNL Tests. . . . . . . . . . . 150 B.83 Predicted Plume Centerline Temperature for the FM/SNL Tests. . . . . . . . . . . 151 B.84 Predicted Plume Centerline Temperature for the FM/SNL Tests. . . . . . . . . . . 152 B.85 Predicted HGL Temperature and Height for the iBMB Single Compartment Tests. . 154 B.86 Predicted Oxygen and Carbon Dioxide for the iBMB Cable Test 5. . . . . . . . . . 154 B.87 Hot Gas Layer Temperature and Height for the NBS Multi-Room Test 100A. . . . 156 B.88 Hot Gas Layer Temperature and Height for the NBS Multi-Room Test 100O. . . . 157 B.89 Hot Gas Layer Temperature and Height for the NBS Multi-Room Test 100Z. . . . . 158 B.90 Hot Gas Layer Temperature and Height for the FM/NBS Four Compartment Test 19.160 B.91 Hot Gas Layer Temperature and Height for the FM/NBS Four Compartment Test 21.161 B.92 Oxygen and Carbon Dioxide Concentration for the FM/NBS Test 19. . . . . . . . . 162 B.93 Oxygen and Carbon Dioxide Concentration for the FM/NBS Test 21. . . . . . . . . 163 B.94 Predicted HGL Temperature and Height for the Seven-Story Hotel Test 7. . . . . . 165 B.95 Predicted Oxygen and Carbon Dioxide for the Seven-Story Hotel Test 7. . . . . . . 166 


\section{List of Tables}

5.1 Thermophysical Properties for VTT Large Hall Tests . . . . . . . . . . . . . . 31

5.2 Test Matrix and Experimental Conditions for NIST NRC Tests $\ldots \ldots \ldots$. . . . . 38

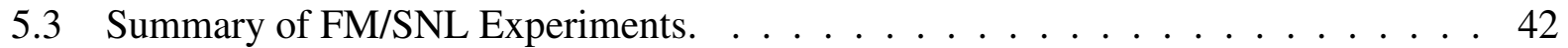

6.1 Relative Difference for HGL Temperature and Depth for Two Measurement Locations in Two Single-Room Tests $\ldots \ldots \ldots \ldots$. . . . . . . . . . 56

8.1 Relative Difference for Oxygen and Carbon Dioxide in Open and Closed Door Tests in a Single Compartment $\ldots \ldots \ldots$. . . . . . . . . . . . . . 69

11.1 Summary of Model Comparisons $\ldots \ldots \ldots$. . . . . . . . . . . . . 82 


\section{Chapter 1}

\section{Overview}

CFAST is a fire model capable of predicting the fire-induced environmental conditions as a function of time for single- or multi-compartment scenarios. Toward that end, the CFAST software calculates the evolution of temperature, smoke, and fire gases throughout a building during a userprescribed fire. The model was developed, and is maintained, by the Fire Research Division of the National Institute of Standards and Technology (NIST).

CFAST is a two-zone model, in that it subdivides each compartment into two zones, or control volumes and the two volumes are assumed to be homogeneous within each zone. This two-zone approach has evolved from observations of layering in actual fires and real-scale fire experiments. Differential equations that approximate solution of the mass and energy balances of each zone, equations for heat conduction into the walls, and the ideal gas law simulate the environmental conditions generated by a fire.

This document describes the underlying structure of the CFAST model and the processes used during the development and deployment of the model. It is intended to provide guidelines for

- the planning for modifications to the model,

- any required reviews for both software and associated documentation of the model,

- testing to be conducted prior to the release of an updated model,

- problem reporting and resolution,

- retention of records, source code, and released software over the life of the code.

Key to ensuring the quality of the software are ongoing validation testing of the model. To this end, a compilation of past and present validation exercises for the CFAST model are presented.

Validation typically involves comparing model simulations with experimental measurements. To say that CFAST is "validated" means that the model has been shown to be of a given level of accuracy for a given range of parameters for a given type of fire scenario. Although the CFAST developers periodically perform validation studies, it is ultimately the end user of the model who decides if the model is adequate for the job at hand. Thus, this Guide does not and cannot be considered comprehensive for every possible modeling scenario. 


\subsection{Software Development and Quality Assurance}

The development and maintenance of CFAST is guided by software quality assurance measures for the planning of modifications to the model that provide required reviews for both software and associated documentation of the model, define testing to be conducted prior to the release of an updated model, describe problem reporting and resolution procedures, and ensure all records, source code, and released software is kept available for the life of the code.

The internal structure of the model also has an impact on the ease of modification and correctness of the model. The method for incorporating new phenomena and ensuring the correctness of the code was adopted as part of the consolidation of CCFM and FAST and has resulted in a more transparent, transportable and verifiable numerical model. This transparency is crucial to a verifiable and robust numerical implementation of the predictive model as discussed in the sections on code checking and numerical analysis. More recently, all of the software development and software tracking has been made available on the web to further enhance the software development process.

\subsection{Model Validation Scenarios}

Key to ensuring the correctness and accuracy of the model are comparisons of the model with both earlier versions of the model and with documented experimental data applicable to the intended range of application of the model. When doing a validation study, the first question to ask is, "What is the application?" There are countless fire scenarios to consider, but from the point of view of validation, it is useful to divide them into two classes - those for which the fire is specified as an input to the model and those for which the fire must be estimated by use of the model. The former is often the case for a design application, the latter for a forensic reconstruction. Consider each in turn.

Design applications typically involve an existing building or a building under design. A socalled "design fire" is prescribed either by a regulatory authority or by the engineers performing the analysis. Because the fire's heat release rate is specified, the role of the model is to predict the transport of heat and combustion products throughout the room or rooms of interest. Ventilation equipment is often included in the simulation, like fans, blowers, exhaust hoods, ductwork, smoke management systems, etc. Sprinkler and heat and smoke detector activation are also of interest. The effect of the sprinkler spray on the fire is usually less of interest since the fire is prescribed rather than predicted. Detailed descriptions of the contents of the building are usually not necessary because these items are not assumed to be burning, and even if they are, the burning rate will be fixed, not predicted. Sometimes, it is necessary to predict the heat flux from the fire to a nearby "target," and even though the target may heat up to some prescribed ignition temperature, the subsequent spread of the fire usually goes beyond the scope of the analysis because of the uncertainty inherent in object to object fire spread.

Forensic reconstructions require the model to simulate an actual fire based on information that is collected after the event, such as eye witness accounts, unburned materials, burn signatures, etc. The purpose of the simulation is to connect a sequence of discrete observations with a continuous description of the fire dynamics. Usually, reconstructions involve more gas/solid phase interaction because virtually all objects in a given room are potentially ignitable, especially when flashover 
occurs. Thus, there is much more emphasis on such phenomena as heat transfer to surfaces, pyrolysis, flame spread, and suppression. In general, forensic reconstructions are more challenging simulations to perform because they require more detailed information about the room geometry and contents, and there is much greater uncertainty in the total heat release rate as the fire spreads from object to object.

CFAST has been applied for both design and reconstruction scenarios. For the former, specified design fires are typically used (e.g., reference [3]). For the latter, iterative simulation with multiple model runs allow the user to develop fire growth inputs consistent with observed post fire conditions.

\subsection{Input Data Required to Run the Model}

All of the data required to run the CFAST model reside in a primary data file, which the user creates. Some instances may require databases of information on objects, thermophysical properties of boundaries, and sample prescribed fire descriptions. In general, the data files contain the following information:

- compartment dimensions (height, width, length)

- construction materials of the compartment (e.g., concrete, gypsum)

- material properties (e.g., thermal conductivity, specific heat, density, thickness, heat of combustion)

- dimensions and positions of horizontal and vertical flow openings such as doors, windows, and vents

- mechanical ventilation specifications

- fire properties (e.g., heat release rate, lower oxygen limit, and species production rates as a function of time)

- sprinkler and detector specifications

- positions, sizes, and characteristics of targets

The CFAST Users Guide [4] provides a complete description of the required input parameters. Some of these parameters have default values included in the model, which are intended to be representative for a range of fire scenarios. Unless explicitly noted, default values were used for parameters not specifically included in this validation study.

\subsection{Property Data}

Required inputs for CFAST must be provided with a number of material properties related to compartment bounding surfaces, objects (called targets) placed in compartments for calculation of 
object surface temperature and heat flux to the objects, or fire sources. For compartment surfaces and targets, CFAST needs the density, thermal conductivity, specific heat, and emissivity.

For fire sources, CFAST needs to know the pyrolysis rate of fuel, the heat of combustion, stochiometric fuel-oxygen ratio, yields of important combustion products in a simplified combustion reaction (carbon monoxide, carbon dioxide, soot, and others), and the fraction of energy released in the form of thermal radiation.

These properties are commonly available in fire protection engineering and materials handbooks. Experimentally determined property data may also be available for certain scenarios. However, depending on the application, properties for specific materials may not be readily available. A small file distributed with the CFAST software contains a database with thermal properties of common materials. These data are given as examples, and users should verify the accuracy and appropriateness of the data.

\subsection{Model Outputs}

Once the simulation is complete, CFAST produces an output file containing all of the solution variables. Typical outputs include (but are not limited to) the following:

- environmental conditions in the room (such as hot gas layer temperature; oxygen and smoke concentration; and ceiling, wall, and floor temperatures)

- heat transfer-related outputs to walls and targets (such as incident convective, radiated, and total heat fluxes)

- fire intensity and flame height

- flow velocities through vents and openings

- detector and sprinkler activation times

Thus, for a given fire scenario, there are a number of different quantities that the model predicts A typical fire experiment can produce hundreds of time histories of point measurements, each of which can be reproduced by the model to some level of accuracy. It is a challenge to sort out all the plots and graphs of all the different quantities and come to some general conclusion. For this reason, this Guide is organized by output quantity, not by individual experiment or fire scenario. In this way, it is possible to assess, over a range of different experiments and scenarios, the performance of the model in predicting a given quantity. Overall trends and biases become much more clear when the data is organized this way.

\subsection{Model Accuracy}

The degree of accuracy for each output variable required by the user is highly dependent on the technical issues associated with the analysis. The user must ask: How accurate does the analysis have to be to answer the technical question posed? Thus, a generalized definition of the accuracy 
required for each quantity with no regard as to the specifics of a particular analysis is not practical and would be limited in its usefulness.

Returning to the earlier definitions of "design" and "reconstruction," fire scenarios, design applications typically are more accurate because the heat release rate is prescribed rather than predicted, and the initial and boundary conditions are far better characterized. Mathematically, a design calculation is an example of a "well-posed" problem in which the solution of the governing equations is advanced in time starting from a known set of initial conditions and constrained by a known set of boundary conditions. The accuracy of the results is a function of the fidelity of the numerical solution, which is largely dependent on the quality of the model inputs. This CFAST validation guide includes efforts to date involving well-characterized geometries and prescribed fires. These studies show that CFAST predictions vary from being within experimental uncertainty to being about $30 \%$ different than measurements of temperature, heat flux, gas concentration, etc (see, for example, reference [5]).

A reconstruction is an example of an "ill-posed" problem because the outcome is known whereas the initial and boundary conditions are not. There is no single, unique solution to the problem. Rather, it is possible to simulate numerous fires that produce the given outcome. There is no right or wrong answer, but rather a small set of plausible fire scenarios that are consistent with the collected evidence and physical laws incorporated into the model. These simulations are then used to demonstrate why the fire behaved as it did based on the current understanding of fire physics incorporated in the model. Most often, the result of the analysis is only qualitative. If there is any quantification at all, it could be in the time to reach critical events, like a roof collapse or room flashover.

\subsection{Uses and Limitations of the Model}

CFAST has been developed for use in solving practical fire problems in fire protection engineering. It is intended for use in system modeling of building and building components. A priori prediction of flame spread or fire growth on objects is not modeled. Rather, the consequences of a specified fire is estimated. It is not intended for detailed study of flow within a compartment, such as is needed for smoke detector siting. It includes the activation of sprinklers and fire suppression by water droplets.

The most extensive use of the model is in fire and smoke spread in complex buildings. The efficiency and computational speed are inherent in the few computation cells needed for a zone model implementation. The use is for design and reconstruction of time-lines for fire and smoke spread in residential, commercial, and industrial fire applications. Some applications of the model have been for design of smoke control systems.

- Compartments: CFAST is generally limited to situations where the compartment volumes are strongly stratified. However, in order to facilitate the use of the model for preliminary estimates when a more sophisticated calculation is ultimately needed, there are algorithms for corridor flow, smoke detector activation, and detailed heat conduction through solid boundaries. This model does provide for non-rectangular compartments, although the application is intended to be limited to relatively simple spaces. There is no intent to include complex geometries where a complex flow field is a driving force. For these applications, computational fluid dynamics (CFD) models are appropriate. 
- Gas Layers: There are also limitations inherent in the assumption of stratification of the gas layers. The zone model concept, by definition, implies a sharp boundary between the upper and lower layers, whereas in reality, the transition is typically over about $10 \%$ of the height of the compartment and can be larger in weakly stratified flow. For example, a burning cigarette in a normal room is not within the purview of a zone model. While it is possible to make predictions within $5 \%$ of the actual temperatures of the gas layers, this is not the optimum use of the model. It is more properly used to make estimates of fire spread (not flame spread), smoke detection and contamination, and life safety calculations.

- Heat Release Rate: CFAST does not predict fire growth on burning objects. Heat release rate is specified by the user for one or more fire objects. The model does include the ability to limit the specified burning based on available oxygen. There are also limitations inherent in the assumptions used in application of the empirical models. As a general guideline, the heat release should not exceed about $1 \mathrm{MW} / \mathrm{m}^{3}$. This is a limitation on the numerical routines attributable to the coupling between gas flow and heat transfer through boundaries (conduction, convection, and radiation). The inherent two-layer assumption is likely to break down well before this limit is reached.

- Radiation: Because the model includes a sophisticated radiation model and ventilation algorithms, it has further use for studying building contamination through the ventilation system, as well as the stack effect and the effect of wind on air circulation in buildings. Radiation from fires is modeled with a simple point source approximation. This limits the accuracy of the model near fire sources. Calculation of radiative exchange between compartments is not modeled.

- Ventilation and Leakage: In a single compartment, the ratio of the area of vents connecting one compartment to another to the volume of the compartment should not exceed roughly $1 / 2 \mathrm{~m}$. This is a limitation on the plug flow assumption for vents. An important limitation arises from the uncertainty in the scenario specification. For example, leakage in buildings is significant, and this affects flow calculations especially when wind is present and for tall buildings. These effects can overwhelm limitations on accuracy of the implementation of the model. The overall accuracy of the model is closely tied to the specificity, care, and completeness with which the data are provided.

- Thermal Properties: The accuracy of the model predictions is limited by how well the user can specify the thermophysical properties. For example, the fraction of fuel which ends up as soot has an important effect on the radiation absorption of the gas layer and, therefore, the relative convective versus radiative heating of the layers and walls, which in turn affects the buoyancy and flow. There is a higher level of uncertainty of the predictions if the properties of real materials and real fuels are unknown or difficult to obtain, or the physical processes of combustion, radiation, and heat transfer are more complicated than their mathematical representations in CFAST.

In addition, there are specific limitations and assumptions made in the development of the algorithms. These are detailed in the discussion of each of these sub-models in the NIST Technical Reference Guide [2]. 


\section{Chapter 2}

\section{Software Quality Assurance}

This chapter describes the processes used during the development and deployment of the Consolidated Fire and Smoke Transport model (CFAST). This software quality assurance (SQA) plan is intended to guide the planning for modifications to the model, provide required reviews for both software and associated documentation of the model, define testing to be conducted prior to the release of an updated model, describe problem reporting and resolution procedures, and ensure all records, source code, and released software is kept available for the life of the code. While this memorandum and many of our practices follow the Institute of Electrical and Electronics Engineers (IEEE) standard for software quality assurance, IEEE 730-2002 [6], other standards have been followed as well. Most notably, ASTM 1355-05, Standard Guide for Evaluating the Predictive Capability of Deterministic Fire Models [1] has been used extensively to guide the documentation, verification, and validation of the model.

CFAST is intended for use only by those competent in the field of fire safety and is intended only to supplement the informed judgment of the qualified user. The software package is a computer model which has limitations based on the way it is used, and the data used for calculations. All results should be evaluated by a qualified user.

The SQA process and requirements outlined in this chapter apply specifically to the CFAST and is focused on ensuring the quality of the numerical predictions of the model. The user interface that may be used to develop input for the model is included in this process to insure that changes to the model are reflected in the user interface and in the data files created by the user interface for use by the model. Of course, users must ensure that the input files developed for the simulations accurately reflect the desired model inputs, whether developed using the supplied user interface, another thirdparty interface, or manually input with a spreadsheet or text editor program. Documentation of these inputs is included as part of the model documentation outlined below.

\subsection{Relevant Publications}

To accompany the model and simplify its use, NIST has developed a Technical Reference Guide [2] and a User's Guide [4] and this Software and Validation Guide. The Technical Reference Guide describes the underlying physical principles and summarizes sensitivity analysis, model validation, and model limitations consistent with ASTM E 1355 [1]. The Users Guide describes how to use the model. 


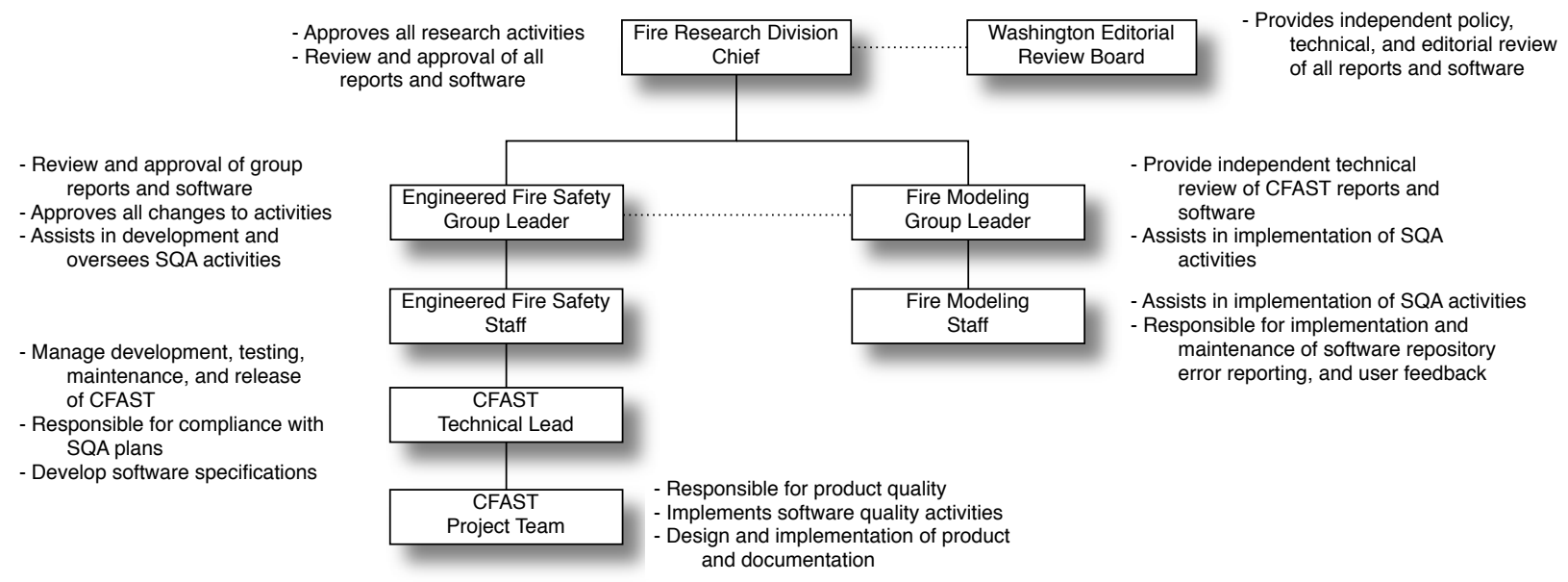

Figure 2.1: CFAST SQA Organization Structure.

The U.S. Nuclear Regulatory Commission has published a verification and validation study of five selected fire models commonly used in support of risk-informed and performance-based fire protection at nuclear power plants [5]. In addition to an extensive study of the CFAST model, the report compares the output of several other models ranging from simple hand calculations to more complex CFD codes such as the Fire Dynamics Simulator (FDS) developed by NIST.

While this document and many of our practices make extensive use of ASTM 1355, Standard Guide for Evaluating the Predictive Capability of Deterministic Fire Models [1] to guide the documentation, verification, and validation of the model, other standards have been followed as well. Most notably, our software quality assurance processes were guided by the IEEE standard for software quality assurance, IEEE 730-2002 [6].

In addition, numerous related documents available at http://cfast.nist.gov provide a wealth of information concerning including earlier versions of the model and its user interface. Software quality assurance (SQA) plan is intended to guide the planning for modifications to the model, provide required reviews for both software and associated documentation of the model, define testing to be conducted prior to the release of an updated model, describe problem reporting and resolution procedures, and ensure all records, source code, and ensure released software is kept available for the life of the code.

\subsection{Model Management}

CFAST is developed and maintained by the Building and Fire Research Laboratory (BFRL) at the National Institute of Standards and Technology (NIST). Like all projects at BFRL, a designated project leader is responsible for directing and prioritizing model development, error correction, and preparation of documentation for the model development. The organization chart in Figure 2.1 provides a graphical representation of the software quality organization structure for CFAST

Review and approval of software and documentation is part of the standard review process for any report or other product developed by NIST. A minimum of five reviews are required prior to 
release of reports or software, including two independent technical peer reviews, two technical and management reviews at the technical workgroup and division level, and a policy review at the NIST-wide level. This review is documented and recorded on the NIST standard form NIST 114 along with official approval notification provided to the primary author of the product.

CFAST is distributed exclusively through a NIST website dedicated to the CFAST model (http://cfast.nist.gov). Content of the website is the responsibility of the CFAST project leader and the BFRL webmaster. Additions and changes to the website are made only with the approval of the CFAST project leader after any required NIST reviews.

\subsection{SQA Documentation}

The released version of CFAST is documented by three primary publications, the Technical Reference Guide[2], the Users Guide [4], and this Software and Model Evaluation Guide. The documents apply to the newest version of the model available on the NIST website. The Technical Reference Guide describes the underlying physical principles, provides a review of model verification and validation efforts by NIST and others, and describes the limitations of the model. The User's Guide describes how to use the model, includes a detailed description of the model inputs and outputs, and provides a process to ensure the correct installation of the model. There are also documents archived on the website that are applicable to older versions of both the model and user interface.

During development of new or corrected features for the model, the following documents are developed:

- Software Requirements and Design Specifications: This is an internal memorandum that documents the intended function of a new or enhanced feature, describes its implementation in sufficient detail to allow an independent review of the feature, and identifies any new or existing testing and validation required prior to acceptance of the feature in a release version of the model. This document forms the basis for necessary changes to the technical reference guide and users guide for the model once the new feature is ready for general release. As defined in IEEE 730-2002 [6], this document includes the software requirements specification, software design description, and software verification and validation plan. The level of detail in this document depends on the complexity of the change to the model.

- Software Validation and Testing Results: This is an internal memorandum that demonstrates the use of the new feature through appropriate test cases and describes validation and verification tests performed to ensure the new feature is implemented properly without unexpected changes in other features. This document forms the basis for the model verification and validation documentation included as part of this Software and Experimental Validation guide. As defined in IEEE 730-2002 [6], this document includes the software verification and validation report. The level of detail in this document depends on the complexity of the change to the model.

Both of these documents are reviewed internally to NIST by group staff not directly involved with model development. In addition, the NIST review process documents the review and approval of released versions of the model as described above. 
Source code for released versions of the model is maintained with version control software that allows tracking of specific changes to the model from version to version. Each version of the model released includes a unique version number that identifies the major and minor version numbers of the release as well as the date of release. Differences with prior versions are documented and provided as part of the release and on the CFAST website so that users can ascertain what effect these changes will have on prior calculations.

\subsection{Standards, Practices, Conventions, and Metrics}

Prior to final implementation of a new feature or change, a review of the proposed modification is conducted by a developer who is not involved in the modification. This review includes review and concurrence of the software requirements and design specification as well as more detailed review of code changes as the complexity of the modification requires. Review and acceptance of the software requirements and design specification by interested project sponsors or users may be included as appropriate. Name and date of approval and/or review is noted electronically in the document.

Review of the testing and validation report is also conducted by a developer who is not involved in the modification prior to proposed model release. Any significant changes in model output (typically a change greater than $1 \%$ of a given output) should be explained based on changes to the code as a result of a new feature. Name and date of approval and/or review is noted electronically in the document.

\subsection{Software Reviews}

Software reviews are outlined as part of the standard practices described above. The standard NIST review process includes review of software and documentation prior to any report or product release by NIST.

\subsection{Model Testing}

Individual testing of model algorithms are made by the developers during any revision of the model. Often, this testing forms the basis for any new standard test cases included with future model releases. System release testing of the model prior to release includes the following:

- Examination of results of test cases specific to any model modifications made as appropriate. Comparison with analytic solutions to simplified problems is desirable when available.

- Examination of results of standard test cases included with the release version of the model. Any changes in the output from prior versions is explained (and documented in the software testing and validation results report) by modifications made to the model.

- For major new releases of the model, a complete suite of test cases should be compared to those from previous versions of the model. At a minimum this includes the set of valida- 
tion exercises described in NUREG 1824 [7], but may include additional example cases or validation exercises as appropriate.

\subsection{Problem Reporting and Resolution}

NIST maintains an e-mail address specifically for inquiries and problem reporting for the CFAST model (cfast@nist.gov). These e-mails are directed to the CFAST project leader for response and resolution as appropriate. Inquiries and responses are catalogued and retained by the project leader.

NIST has developed an automated reporting and resolution tracking website for use with the CFAST model to facilitate tracking and cataloging of inquires, problems, and model enhancements / revisions. This is included as part of the CFAST website at http://cfast.nist.gov

\subsection{Tools, Techniques, and Methodologies}

NIST will use an automated comparison tool (under development) to compare CFAST predictions between different versions of the model and with experimental data to simplify testing and validation for the CFAST model.

\subsection{Media Control}

Release versions of the CFAST model are available exclusively on the CFAST specific website maintained by the Building and Fire Research Laboratory (BFRL) at NIST. This website is included in NISTs automated backup and recovery system for computer systems organization wide.

Development versions of the model are maintained by the CFAST project leader. All software and documents are included in NISTs automated backup and recovery system for computer systems organization wide. As part of its model development, NIST maintains a web-based system for version control and history of both the CFAST source code and of pre-release and release executables for the software.

These computer systems are available only to specified personnel, including the CFAST project leader and project team members.

\subsection{Supplier Control}

CFAST is entirely a product of BFRL / NIST and as such does not include any commercial software libraries. The differential equation solver used by CFAST, DASSL, is a publicly available software package. Full source code for the solver as used by CFAST is maintained under version control with the rest of the model code.

BFRL currently uses Microsoft Visual Studio 2010 and Intel Fortran XE 2011 for development ${ }^{1}$. Prior to any change to a different development system, a full test suite of test cases are

\footnotetext{
${ }^{1}$ Certain commercial entities, equipment, or materials may be identified in this document in order to describe an experimental procedure or concept adequately. Such identification is not intended to imply recommendation or endorsement by the National Institute of Standards and Technology, nor is it intended to imply that the entities,
} 
compared to verify consistent operation of the model and model results.

\subsection{Records Collection, Maintenance, and Retention}

All software, documentation, and SQA documents are retained by the CFAST project leader, typically in electronic form. Software and documentation is also maintained and archived on the NIST CFAST website as part of the version control software for the model.

BFRL management approval is required prior to destruction of old or out-of-date records. Records are typically maintained for a minimum of 25 years.

\subsection{Training}

No specific training is identified for use of this SQAP. Details of training requirements for use of the model included in the CFAST users guide is applicable to developers of the model as well.

\subsection{Risk Management}

The primary risk management tool for software developed and released by NIST is the official NIST review process for software, documents, and other products of NIST. Official approval is required prior to release of the model for general use.

materials, or equipment are necessarily the best available for the purpose. 


\section{Chapter 3}

\section{Software Structure and Robustness}

The mathematical and numerical robustness of a deterministic computer model depends upon three issues: the code must be transparent so that it can be understood and modified by visual inspection; it must be possible to check and verify with automated tools; and there must be a method for checking the correctness of the solution, at least for asymptotic (steady state) solutions (numerical stability and agreement with known solutions). In order to understand the meaning of accuracy and robustness, it is necessary to understand the means by which the numerical routines are structured. In this chapter, details of the implementation of the model are presented, including the tests used to assess the numerical aspects of the model. These include:

- the structure of the model, including the major routines implementing the various physical phenomena included in the model,

- the organization of data initialization and data input used by the model,

- the structure of data used to formulate the differential equations solved by the model,

- a summary of the main control routines in the model that are used to control all input and output, initialize the model and solve the appropriate differential equation set for the problem to be solved,

- the means by which the computer code is checked for consistency and correctness,

- analysis of the numerical implementation for stability and error propagation, and

- comparison of the results of the system model with simple analytical or numerical solutions.

\subsection{Structure of the Numerical Routines}

A methodology which is critical to verification of the model is the schema used to incorporate physical phenomena. This is the subroutine structure discussed below. The method for incorporating new phenomena and ensuring the correctness of the code was adopted as part of the consolidation of CCFM and FAST. This consolidation occurred in 1990 and has resulted in a more transparent, transportable and verifiable numerical model. This transparency is crucial to a verifiable and robust 


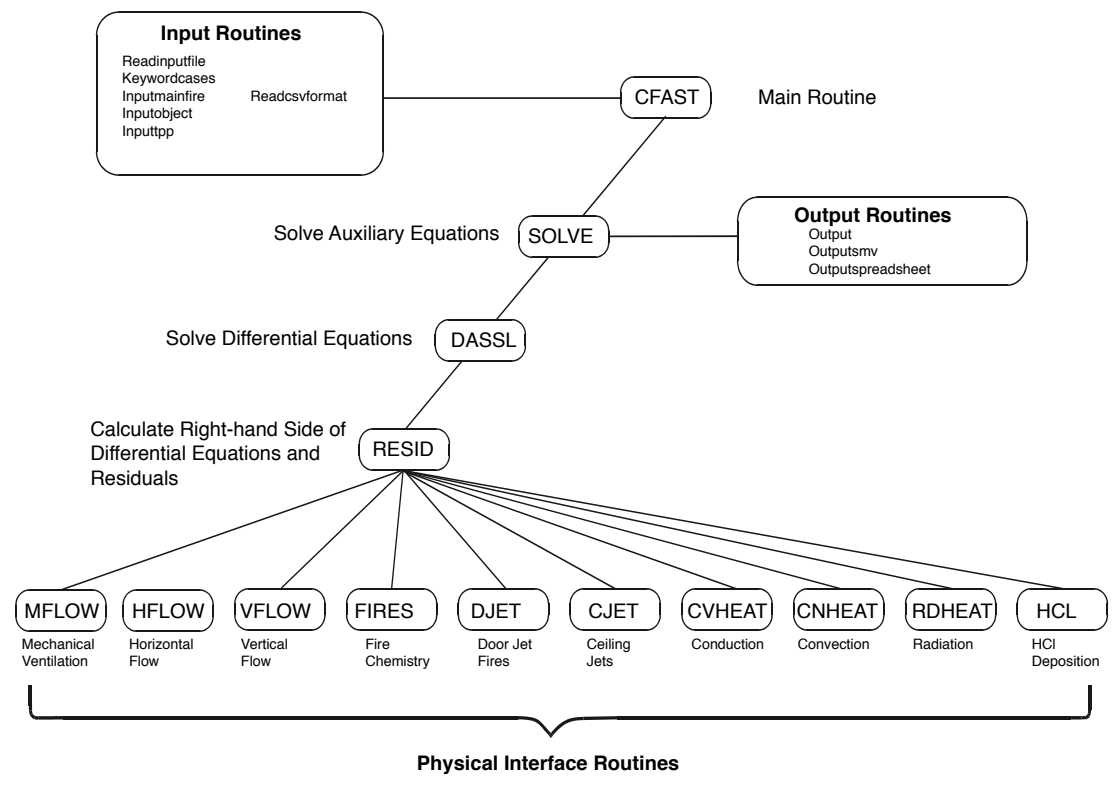

Figure 3.1: Subroutine structure for the CFAST model showing major routines and calling structure.

numerical implementation of the predictive model as discussed in the sections on code checking and numerical analysis.

The model can be split into distinct parts. There are routines for reading data, calculating results and reporting the results to a file or printer. The major routines for performing these functions are identified in figure 3.1. These physical interface routines link the CFAST model to the actual routines which calculate quantities such as mass or energy flow at one particular point in time for a given environment.

The routines SOLVE, RESID and DASSL are the key to understanding how the physical equations are solved. SOLVE is the control program that oversees the general solution of the problem. It invokes the differential equation solver DASSL [8] which in turn calls RESID to solve the transport equations. Given a solution at time $t$, what is the solution at time t plus a small increment of time, $\delta t$ ? The differential equations are of the form

$$
\begin{aligned}
\frac{d y}{d x} & =f(y, t) \\
y\left(t_{0}\right) & =y_{0}
\end{aligned}
$$

where $y$ is a vector representing pressure, layer height, mass and such, and $f$ is a vector function that represents changes in these values with respect to time. The term $y_{0}$ is an initial condition at the initial time $t_{0}$. The time increment is determined dynamically by the program to ensure convergence of the solution at $t+\Delta t$. The subroutine RESID computes the right hand side of eq 3.1 and returns a set of residuals of that calculation to be compared to the values expected by DASSL. DASSL then checks for convergence. Once DASSL reaches an error limit (defined as convergence of the equations) for the solution at $t+\Delta t$, SOLVE then advances the solution 
of species concentration, wall temperature profiles, and mechanical ventilation for the same time interval. Note that there are several distinct time scales that are involved in the solution of this type of problem. The fastest will be chemical kinetics. In CFAST, chemical reactions are assume to be instantaneous so we ignore the impact of chemical kinetics. The next larger time scale is that associated with the flow field. These are the equations which are cast into the form of ordinary differential equations. Then there is the time scale for mechanical ventilation, and finally, heat conduction through objects.

Chemical kinetic times are typically on the order of milliseconds. The transport time scale are on the order of $0.1 \mathrm{~s}$. The mechanical ventilation and conduction time scales are typically several seconds, or even longer. The time step is dynamically adjusted to a value appropriate for the solution of the currently defined equation set. In addition to allowing a more correct solution to the pressure equation, very large time steps are possible if the problem being solved approaches steady-state.

\subsection{Comparison with Analytic Solutions}

Certain CFAST sub-models address phenomena that have analytical solutions, for example, one dimensional heat conduction through a solid or pressure increase in a sealed or slightly leaky compartment as a result of a fire or fan. The developers of CFAST use analytical solutions to test sub-models to verify the correctness of the coding of the model as part of the development. Such verification efforts are relatively simple and the results may not always be published or included in the documentation. Two additional types of verification are possible. The first type, discussed in Section 3, Theoretical Basis, involves validating individual algorithms against experimental work. The second involves simple experiments, especially for conduction and radiation, for which the results are asymptotic (e.g., a simple single-compartment test case with no fire, all temperatures should equilibrate asymptotically to a single value). Such comparisons are common and not usually published.

\subsection{Code Checking}

Two standard programs have been used to check the CFAST model structure and language. Specifically, FLINT and LINT have been applied to the entire model to verify the correctness of the interface, undefined or incorrectly defined (or used) variables and constants, and completeness of loops and threads.

The CFAST code has also been checked by compiling and running the model on a variety of computer platforms. Because FORTRAN and C are implemented differently for various computers, this represents both a numerical check as well as a syntactic check. CFAST has been compiled for Sun (Solaris), SGI (Irix), Microsoft Windows-based PCs (Lahey, Digital, and Intel FORTRAN), and Concurrent computer platforms. Within the precision afforded by the various hardware implementations, the model outputs are identical on the different platforms. 1

\footnotetext{
${ }^{1}$ Typically an error limit of one part in $10^{6}$ which is the limit set for the differential equation solver in the solution of the CFAST equations
} 
The CFAST Technical Reference Guide [2] contains a detailed description of the CFAST subroutine structure and interactions between the subroutines.

\subsection{Numerical Tests}

CFAST is designed to use 64-bit precision for real number calculations to minimize the effects of numerical error.

The differential and algebraic equation solver (called DASSL) has been tested for a variety of differential equations and is widely used and accepted [8]. The radiation and conduction routines have also been tested against known solutions for asymptotic results [9].

Coupling between the physical algorithms of the model and the differential equation solver also works to ensure numerical accuracy by dynamically adjusting the time step used by the model to advance the solutions of the equation set. Solution tolerances are set to require solution of the model equations within one part in $10^{6}$. This ensures that the error attributable to numerical solution is far less than that associated with the model assumptions. 


\section{Chapter 4}

\section{Survey of Past Validation Work}

CFAST has been subjected to extensive validation studies by NIST and others. There are two ways of comparing predictive capability with actual events. The first is simply graphing the time series curves of model results with measured values of variables such as temperature. Another approach is to consider the time to critical conditions such as flashover. Making such direct comparisons between theory and experiment provides a sense of whether predictions are reasonable. This chapter provides a review of CFAST validation efforts by NIST and others to better understand the quality of the predictions by the model.

Some of the work has been performed at NIST, some by its grantees and some by engineering firms using the model. Because each organization has its own reasons for validating the model, the referenced papers and reports do not follow any particular guidelines. Some of the works only provide a qualitative assessment of the model, concluding that the model agreement with a particular experiment is "good" or "reasonable." Sometimes, the conclusion is that the model works well in certain cases, not as well in others. These studies are included in the survey because the references are useful to other model users who may have a similar application and are interested in qualitative assessment. It is important to note that some of the papers point out flaws in early releases of CFAST that have been corrected or improved in more recent releases. Some of the issues raised, however, are still subjects of active research. Continued updates for CFAST are greatly influenced by the feedback provided by users, often through publication of validation efforts.

\subsection{Comparisons with Full-Scale Tests Conducted Specifically for the Chosen Evaluation}

Several studies have been conducted specifically to validate the use of CFAST in building performance design. Dembsey [10] used CFAST version 3.1 to predict the ceiling jet temperatures, surface heat fluxes and heat transfer coefficients for twenty compartment fire experiments in a compartment that is similar in size, geometry, and construction to the standard fire test compartment specified in the Uniform Building Code [11] Results from $330 \mathrm{~kW}, 630 \mathrm{~kW}$, and $980 \mathrm{~kW}$ fires were used. In general, CFAST made predictions which were higher than the experimental

\footnotetext{
${ }^{1}$ The 1997 Uniform Building Code has been superceded by the International Building Code, 2003 Edition, International Code Council, Country Club Hills, Illinois.
} 
results. In these cases, the temperature prediction is typically $20 \%$ to $30 \%$ higher than measured values. Much of this can be attributed to not knowing the species production (soot) and relative absorption of radiation by the gas layers which highlights the importance of scenario specification. This is the most common cause of over prediction of temperature by CFAST. A secondary source of discrepancy is correcting for radiation from thermocouple beads. The authors provide for this correction, but the corrections cited are not as large as has been reported in other fire experiments [12].

He et al. [13] describe a series of full-scale fire experiments that were designed to investigate the validity of two zone models including CFAST version 3.1. The experiments, involving steady state burning rates and a number of ventilation conditions, were conducted in a four-story building. Temperature, pressure, flow velocity, smoke density and species concentrations were measured in various parts of the building. The stack effect and its influence on temperature distribution in a stair shaft were observed. Comparisons were then made between the experimental results and the model predictions. Early in the fire there is a few percent difference ${ }^{2}$ between the predictions and measurements; beyond $10 \mathrm{~min}$, there are significant variations. Both the experiment and the model are internally consistent; that is, higher flow leads to a higher interface height (figure 13 in the paper). Once again, the difference is about $25 \%$. The authors discuss the effect of fuel composition and correction for radiation from thermocouple beads but did not draw firm conclusions based on their measurements of fuel products.

A series of experimental results for flaming fires, obtained using realistic fires in a prototype apartment building were performed by Luo et al. [14]. Fuel configurations in the fire test included a horizontal plain polyurethane slab, mock-up chair (polyurethane slabs plus a cotton linen cover), and a commercial chair. CFAST version 3.1 typically over-predicted upper layer temperatures by $10 \%$ to $50 \%$ depending on the test conditions and measurement location in that test. The predicted and experimental time dependent upper layer temperatures were similar in shape. The time to obtain peak upper layer temperatures was typically predicted to within $15 \%$ of the experimental measurements. The authors concluded that CFAST was conservative in terms of life safety calculations.

In order to optimize fire service training facilities, the best use of resources is imperative. The work reported by Poole et al. [15] represents one aspect of a cooperative project between the city of Kitchener Fire Department (Canada) and the University of Waterloo aimed at developing design criteria for the construction of a fire fighter training facility. One particular criterion is that realistic training with respect to temperature, heat release and stratification be provided in such a facility. The purpose of this paper was to compare existing analytical heat release and upper and lower gas temperature rise correlations and models with data from actual structures which were instrumented and burned in collaboration with the Kitchener Fire Department. According to the authors, the CFAST model was used 'successfully' to predict these conditions and will be used in future design of such facilities.

A report by Bailey et al. [16] compares predictions by CFAST version 3.1 to data from real scale fire tests conducted onboard ex-USS SHADWELL, the Navy's R\&D damage control platform. The phenomenon of particular interest in this validation series was the conduction of heat in the vertical direction through compartment ceilings and floors. As part of this work, Bailey et al. [17] compared CFAST temperature predictions on the unexposed walls of large metal

\footnotetext{
${ }^{2}$ Unless otherwise noted, percent differences are defined as (model-experiment)/experiment $\mathrm{x} 100$.
} 
boxes, driven by steady state fires. This tested the models prediction of radiation and conduction in both the vertical and horizontal directions. Indirectly it quantifies the quality of the conduction/convection/radiation models. The model and experiment compared well within measurement error bounds of each. The comparison was particularly good for measurements in the fire compartment as well as for the compartment and deck directly above it, with predictions typically agreeing with experiments within measurement uncertainty. The model under-predicted the temperatures of the compartments and decks not directly adjacent to the fire compartment early in the tests. Most of the error arose due to uncertainty in modeling the details of the experiment. The size of the vent openings between decks and to the outside must be included, but these were not always known. Cracks formed in the deck between the fire compartment and the compartment above due to the intense fire in the room of origin, but a time dependent record was not kept. The total size of the openings to the outside of warped doors in both compartments was not recorded. As can be seen in figures 7 and 8 of reference [16], the steady state predictions are identical (within error bounds of the experiment and prediction). The largest error is after ignition (uncertainty in the initial fire) and during development of the cracks between the compartments. While this does not affect the agreement in the room of origin, it does lead to an uncertainty of about $30 \%$ in the adjacent compartment.

\subsection{Comparisons with Previously Published Test Data}

A number of researchers have studied the level of agreement between computer fire models and real-scale fires. These comparisons fall into two broad categories: fire reconstruction and comparison with laboratory experiments. Both categories provide a level of verification for the models used. Fire reconstruction, although often more qualitative, provides a higher degree of confidence for the user when the models successfully simulate real-life conditions. Comparisons with laboratory experiments, however, can yield detailed comparisons that can point out weaknesses in the individual phenomena included in the models.

Deal [18] reviewed four computer fire models (CCFM [19], FIRST [20], FPETOOL [21] and FAST [22] version 18.5 (the immediate predecessor to CFAST)) to ascertain the relative performance of the models in simulating fire experiments in a small room (about $12 \mathrm{~m}^{3}$ in volume) in which the vent and fuel effects were varied. Peak fire size in the experiments ranged up to 800 $\mathrm{kW}$. According to the author, all the models simulated the experimental conditions including temperature, species generation, and vent flows 'quite satisfactorily.' With a variety of conditions, including narrow and normal vent widths, plastic and wood fuels, and flashover and sub-flashover fire temperatures, competence of the models at these room geometries was 'demonstrated.'

\subsubsection{Fire Plumes}

Davis compared predictions by CFAST version 5 (and other models) for high ceiling spaces [23]. In this paper, the predictive capability of two algorithms designed to calculate plume centerline temperature and maximum ceiling jet temperature in the presence of a hot upper layer were compared to measurements from experiments and to predictions using CFASTs ceiling jet algorithm. The experiments included ceiling heights of $0.58 \mathrm{~m}$ to $22 \mathrm{~m}$ and heat release rates of $0.62 \mathrm{~kW}$ to $33 \mathrm{MW}$. When compared to the experimental results CFASTs ceiling jet algorithm tended to 
over-predict the upper layer temperature by $20 \%$. With proper adjustment for radiation effects in the thermocouple measurements, some of this difference disappears. The effect of entrainment of the upper layer gases was identified for improvement.

\subsubsection{Multiple Compartments}

Jones and Peacock [24] presented a limited set of comparisons between the FAST model (version 18.5) and a multi-room fire test. The experiment involved a constant fire of about $100 \mathrm{~kW}$ in a three-compartment configuration of about $100 \mathrm{~m}^{3}$. They observed that the model predicted an upper layer temperature that was too high by about $20 \%$ with satisfactory prediction of the layer interface position. These observations were made before the work of Pitts et al. [12] showed that the thermocouple measurements need to be corrected for radiation effects. Convective heating and plume entrainment were seen to limit the accuracy of the predictions. A comparison of predicted and measured pressures in the rooms showed within $20 \%$. Since pressure is the driving force for flow between compartments, this agreement was seen as important.

Levine and Nelson [25] used a combination of full-scale fire testing and modeling to simulate a fire in a residence. The 1987 fire in a first-floor kitchen resulted in the deaths of three persons in an upstairs bedroom, one with a reported blood carboxyhemoglobin content of $91 \%$. Considerable physical evidence remained. The fire was successfully simulated at full scale in a fully-instrumented seven-room two-story test structure. The data collected during the test have been used to test the predictive abilities of two multiroom computer fire models: FAST and HARVARD VI. A coherent ceiling layer flow occurred during the full-scale test and quickly carried high concentrations of carbon monoxide to remote compartments. Such flow is not directly accounted for in either computer code. However, both codes predicted the carbon monoxide buildup in the room most remote from the fire. Prediction of the pre-flashover temperature rise was also 'good' according to the authors. Prediction of temperatures after flashover that occurred in the room of fire origin was seen as 'less good.' Other predictions of conditions throughout the seven test rooms varied from 'good approximations' to 'significant deviations' from test data. Some of these deviations are believed to be due to combustion chemistry in the not upper layer not considered in detail in either of the two models.

\subsubsection{Large Compartments}

Duong [26] studied the predictions of several computer fire models (CCFM, FAST, FIRST, and BRI [27]), comparing the models with one another and with large fires (4 MW to $36 \mathrm{MW}$ ) in an aircraft hanger $\left(60000 \mathrm{~m}^{3}\right)$. For the $4 \mathrm{MW}$ fire size, he concluded that all the models are 'reasonably accurate.' At $36 \mathrm{MW}$, however, 'none of the models did well.' Limitations of the heat conduction and plume entrainment algorithms were thought to account for some of the inaccuracies.

\subsubsection{Prediction of Flashover}

A chaotic event that can be predicted by mathematical modeling is that of flashover. Flashover is the common term used for the transition a fire makes from a few objects pyrolyzing to full room involvement. It is of interest to the fire service because of the danger to fire fighters and to building 
designers because of life safety and the attendant impact on occupants. Several papers have looked at the capability of CFAST to predict the conditions under which flashover can occur.

Chow [28] concluded that FAST correctly predicted the onset of flashover if the appropriate criteria were used. The criteria were gas temperature near the ceiling, heat flux at the floor level and flames coming out of the openings. This analysis was based on a series of compartment fires.

A paper by Luo et al. [29] presents a comparison of the results from CFAST version 3 against a comprehensive set of data obtained from one flashover fire experiment. The experimental results were obtained from a full-scale prototype apartment building under flashover conditions. Three polyurethane mattresses were used as fuel. It was found that the predicted temperatures from the CFAST fire model agreed well with the experimental results in most areas, once radiation corrections are applied to the thermocouple data.

Collier [30] makes an attempt to quantify the fire hazards associated with a typical New Zealand dwelling with a series of experiments. These tests, done in a three-bedroom dwelling, included both non-flashover and flashover fires. The predictions by CFAST version 2 were seen by the author as consistent with the experiments within the uncertainty of each.

Post-flashover fires in shipboard spaces have a pronounced effects on adjacent spaces due to highly conductive boundaries. CFAST (version 3.1) predictions for the gas temperature and the cold wall temperature were compared with shipboard fires [31]. The comparisons between the model and experimental data show 'conservative predictions' according to the authors. The authors attribute this to an overestimation of the average hot wall temperature and an underestimation of external convective losses due to wind effects.

Finally, a comparison of CFAST with a number of simple correlations was used by Peacock and Babrauskas [32, 33] to simulate a range of geometries and fire conditions to predict the development of the fire up to the point of flashover. The simulations represent a range of compartment sizes and ceiling heights. Both the correlations and CFAST predictions were seen to provide a lower bound to observed occurrence of flashover. For very small or very large compartment openings, the differences between the correlations, experimental data, and CFAST predictions was more pronounced.

The important test of all these prediction methods is in the comparison of the predictions with actual fire observations. Figure 4.1 (reference [33]) presents estimates of the energy required to achieve flashover for a range of room and vent sizes. This figure is an extension of the earlier work of Babrauskas [34] and includes additional experimental measurements from a variety of sources, most notably the work of Deal and Beyler [35]. For a number of the experimental observations, values are included that were not explicitly identied as being a minimum value at flashover. In addition, figure 4.1 includes predictions from the CFAST model (version 5).

As with some of the experimental data defining flashover as an upper layer temperature reaching $600{ }^{\circ} \mathrm{C}$, many experimental measures were reported as peak values rather than minimum values necessary to achieve flashover. Thus, ideally all the predictions should provide a lower bound for the experimental data. Indeed, this is consistent with the graph the vast majority of the experimental observations lie above the correlations and model predictions. For a considerable range in the ratio $A_{T} / A \sqrt{h}$, the correlations of Babrauskas [34] Thomas [36], and the MQH correlation of McCaffrey et al. [37] provide similar estimates of the minimum energy required to produce flashover. The estimates of Hägglund [38] yields somewhat higher estimates for values of $A_{T} / A \sqrt{h}$ greater than $20 \mathrm{~m}^{-1 / 2}$.

The results from the CFAST model for this single compartment scenario provide similar results 


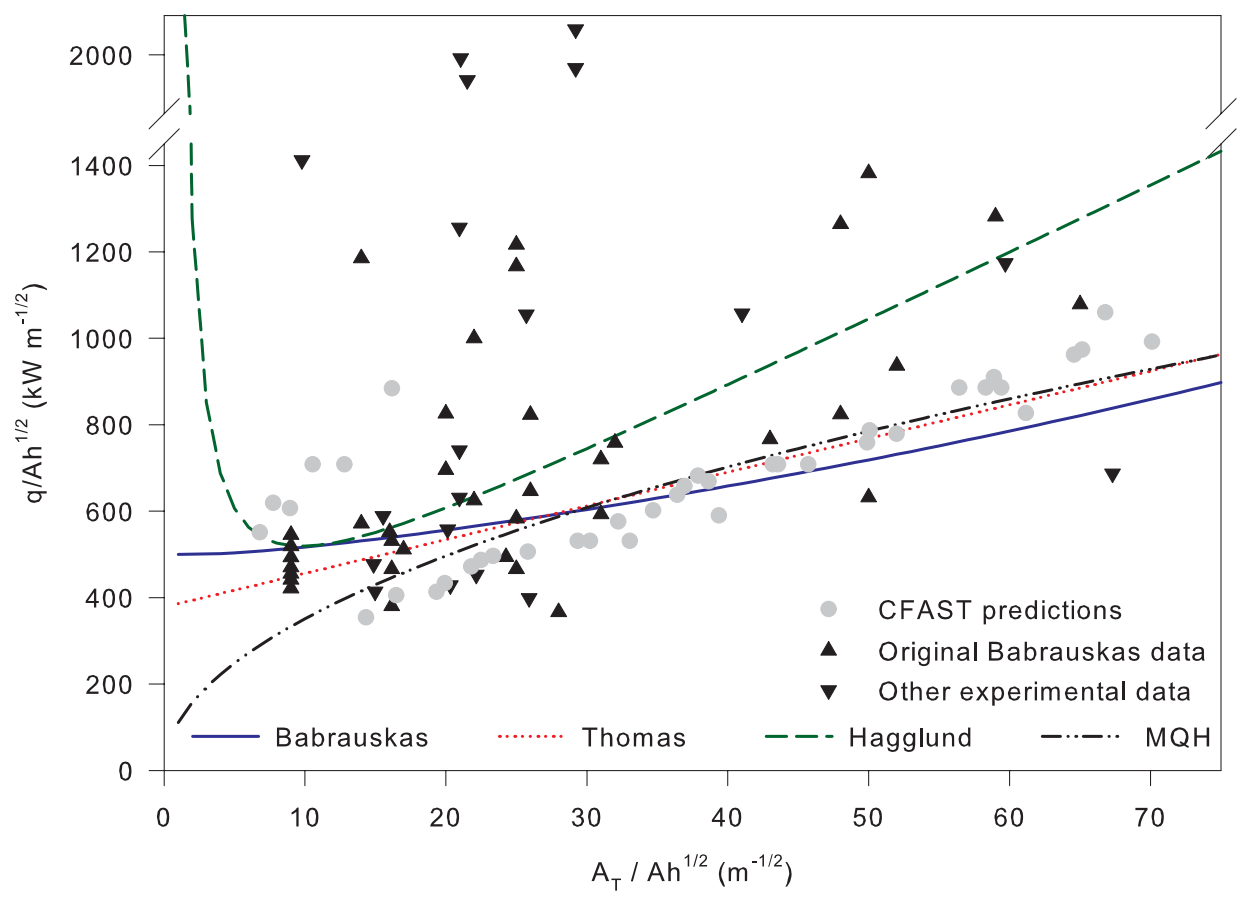

Figure 4.1: Comparison of correlations, CFAST predictions, and experimental data for the prediction of flashover in a compartment fire. 
to the experiments and the correlations for most of the range of $A_{T} / A \sqrt{h}$. For small values of $A_{T} / A \sqrt{h}$, the CFAST values rise somewhat above the values from the correlations. These small values of $A_{T} / A \sqrt{h}$ result from either very small compartments (small $A_{T}$ ) or very large openings (large $A_{T} / A \sqrt{h}$ ), both of which stretch the limits of the assumptions inherent in the model. For very small compartments, radiation from the fire to the compartment surfaces becomes more important, enhancing the conductive heat losses through the walls. However, the basic two-zone assumption may break down as the room becomes very small. For very large openings, the calculation of vent flow via an orifice flow coefficient approach is likely inaccurate. Indeed, for such openings, this limitation has been observed experimentally [34]. The estimates are close to the range of uncertainty shown by the correlations which also diverge at very small values of $A_{T} / A \sqrt{h}$.

Perhaps most significant in these comparisons is that all the simple correlations provide estimates similar to the CFAST model and all the models are consistent with a wide range of experimental data. For this simple scenario, little is gained with the use of the more complex models. For more complicated scenarios, the comparison may not be as simple.

\subsection{Comparison with Documented Fire Experience}

There are numerous cases of CFAST being used to adjudicate legal disputes. Since these are discussed in courts of law, there is a great deal of scrutiny of the modeling, assumptions, and results. Most of these simulations and comparisons are not available in the public literature. A few of the cases which are available are discussed below. The metric for how well the model performed is its ability to reproduce the time-line as observed by witnesses and the death of occupants or the destruction of property as was used in evidence in legal proceedings.

As mentioned in section 4.2.2, Levine and Nelson describe the use of FAST for understanding the deaths of two adults in a residence in Sharon, Pennsylvania in 1987 [25]. The paper compared the evidence of the actual fire, a full scale mockup done at NIST and the results from FAST (version 18) [39] and Harvard VI [40]. The most notable shortcoming of the models was the lower than actual temperatures in the bedrooms, caused by loss of heat through the fire barriers. This led to the improvement in CFAST in the mid-90s to couple compartments together so that both horizontal and vertical heat transfer can occur to adjacent compartments.

Bukowski used CFAST version 3.1 to analyze a fire in New York City [41] in 1994 which resulted in the death of three fire fighters. The CFAST model was able to reproduce the observed conditions and supported the theory as to how the fire began and the cause of death of the three fire fighters.

Chow describes the use and comparison of CFAST simulations with a 1996 high rise building fire in Hong Kong [42]. CFAST simulations were performed to help understand the probable fire environment under different conditions. Three simulations were performed to study the consequences of a fire starting in the lift shaft. Smoke flow in the simulations qualitatively matched those observed during the incident.

In the early morning hours of March 25,1990 a tragic fire took the lives of 87 persons at a neighborhood club in the Bronx, New York [43]. The New York City Fire Department requested the assistance of the NIST Center for Fire Research (CFR) in understanding the factors which contributed to this high death toll and to develop a strategy that might reduce the risk of a similar occurrence in the many similar clubs operating in the city. The simulation showed the potential for 
development of untenable conditions within the club and particularly in the single exit stairway.

\subsection{Comparison with Experiments Which Cover Special Situ- ations}

There are several sets of comparisons used in the development of the model or specific applications beyond those discussed more generally above.

\subsubsection{Nuclear Facilities}

Floyd validated CFAST version 3.1 by comparing the modeling results with measurements from fire tests at the Heiss-Dampf Reaktor (HDR) facility [44]. The structure was originally the containment building for a nuclear power reactor in Germany. The cylindrical structure was $20 \mathrm{~m}$ in diameter and $50 \mathrm{~m}$ in height topped by a hemispherical dome $10 \mathrm{~m}$ in radius. The building was divided into eight levels. The total volume of the building was approximately $11000 \mathrm{~m}^{3}$. From 1984 to 1991, four fire test series were performed within the HDR facility. The T51 test series consisted of 11 propane gas tests and three wood crib tests. To avoid permanent damage to the test facility, a special set of test rooms were constructed, consisting of a fire room with a narrow door, a long corridor wrapping around the reactor vessel shield wall, and a curtained area centered beneath a maintenance hatch. The fire room walls were lined with fire brick. The doorway and corridor walls had the same construction as the test chamber. Six gas burners were mounted in the fire room. The fuel source was propane gas mixed with $10 \%$ air fed at a constant rate to one of the six burners.

In general, the comparison between CFAST and the HDR results was seen as 'good' by the author, with two exceptions. The first is the over estimate of the temperature of the upper layer, typically within about $15 \%$ of the experimental measurements. This is common and generally results from using too low a value for the production of soot, water (hydrogen) and carbon monoxide. The other exception consists of predictions in spaces where the zone model concept breaks down, for example in the stairways between levels. In this case, CFAST has to treat the space either in the filling mode (two layer approximation) or as a fully mixed zone (using the SHAFT option). Neither is quite correct, and in order to understand the condition in such spaces in detail (beyond the transfer of mass and energy), a more detailed CFD model must be used, for example, FDS [45].

The U.S. Nuclear Regulatory Commission performed an extensive verification and validation of several fire models commonly used in nuclear power plant applications [7]. These models included simple spreadsheet calculations, zone models (including CFAST [5]), and CFD models. The results of this study are presented in the form of relative differences between fire model predictions and experimental data for fire modeling attributes such as temperature or heat flux that are important to NPP fire modeling applications. These relative differences are affected by the capabilities of the models, the availability of accurate applicable experimental data, and the experimental uncertainty of these data. Evaluation of the two-zone models showed that the models simulated the experimental results within experimental uncertainty for many of the parameters of interest. The reason for this may be that the relatively simple experimental configurations selected for this study 
conform well to the simple two-layer assumption that is the basis of these models.

While the relative differences sometimes show agreement for many parameters, they also show both under-prediction and over-prediction in some circumstances, most notably when conditions vary within a compartment or detailed local conditions are important to accurate prediction (for example, plume temperature or heat flux near to the fire source). The results and comparisons included the the NRC study are included in this report for the current version of CFAST.

\subsubsection{Small Scale Testing}

As an implementation of the zone model concept, CFAST is applicable to a wide range of scenarios. One end of this spectrum are small compartments, one to two meters on a side. Several research efforts have looked at small scale validation. There are three papers by Chow [46, 47, 48] which examine this issue. The first is the use of an electric heater with adjustable thermal power output was to verify temperature predictions by CFAST version 3.1. The second was closed chamber fires studied by burning four types of organic liquids, namely ethanol, N-heptane, and kerosene. The burning behavior of the liquids was observed, and the hot gas temperature measured. These behaviors along with the transient variations of the temperature were then compared with those predicted by the CFAST model. Finally, in another series of experiments, three zone models, one of which was CFAST, were evaluated experimentally using a small fire chamber. Once again, liquid fires were chosen for having better control on the mass loss rate. The results on the development of smoke layer and the hot gas temperature predicted by the three models were compared with those measured experimentally. According to Chow, 'fairly good agreement' was found if the input parameters were carefully chosen.

\subsubsection{Unusual Geometry and Specific Algorithms}

A zone model is inherently a volume calculation. There is an assumption in the derivation of the equations that gas layers are strongly stratified. This allows for the usual interpretation that a volume can then be thought of as a rectangular parallelepiped, which allows the developers to express the volume in terms of a floor area and height of a compartment, saying simply that the height times the floor area is the volume. However, there are other geometries which can be adequately described by zone models. Tunnels, ships, and attics are the most common areas of application which fall outside of the usual scope.

\section{Railway and Vehicle Tunnels}

Altinakar et al. [49] used a modified version of CFAST for predicting fire development and smoke propagation in vehicle or railroad tunnels. The two major modifications made to the model dealt with mixing between the upper and lower layers and friction losses along the tunnel. The model was tested by simulating several full-scale tests carried out at memorial Tunnel Ventilation Test Program in West Virginia, and the Offeneg Tunnel in Switzerland. His article compares simulated values of temperature, opacity and similar sensible quantities with measured values and discusses the limits of the applicability of zone models for simulating fire and smoke propagation in vehicle and railroad tunnels. 
Peacock et al. [50] compared times to untenable conditions determined from tests in a passenger rail car with those predicted by CFAST for the same car geometry and fire scenarios. For a range of fire sizes and growth rates, they found agreement that averaged approximately $13 \%$.

\section{Non-Uniform Compartments}

In January 1996, the U.S. Navy began testing how the CFAST model would perform when tasked with predicting shipboard fires. These conditions include mass transport through vertical vents (representing hatches and scuttles), energy transport via conduction through decks, improvement to the radiation transport sub-model, and geometry peculiar to combat ships. The purpose of this study was to identify CFAST limitations and develop methods for circumnavigating these problems [51]. A retired ship representing the forward half of a USS Los Angeles class submarine was used during this test. Compartments in combat ships are not square in floor area, nor do they have parallel sides.

Application of CFAST to these scenarios required a direct integration of compartment crosssectional area as a function of height to correctly interpret the layer interface position and provide correct predictions for flow through doors and windows (vertical vents). This required user specification of the area as a function of height (ROOMA and ROOMH inputs) to provide a description for the model to use. For most applications of CFAST, the effort required for the input outweighs any additional precision in the calculated results gained by use of the ROOMA and ROOMH inputs in the model.

\section{Long Corridors}

Prior to development of the corridor flow model, the implementation of flow in compartments assumed that smoke traveled instantly from one side of a compartment to another. The work of Bailey et al. [52] provided the basis for the corridor flow model in CFAST. According to the author, it shows 'good agreement' for the delay time calculated using CFAST version 5 and measured flow along high aspect ratio passageways.

\section{Mechanical Ventilation}

There have been two papers which have looked at the effectiveness of the mechanical ventilation system. The first considered a fire chamber of length $4.0 \mathrm{~m}$, width $3.0 \mathrm{~m}$ and height $2.8 \mathrm{~m}$ with adjustable ventilation rates [53]. Burning tests were carried out with wood cribs and methanol to study the preflashover stage of a compartmental fire and the effect of ventilation. The mass loss rate of fuel, temperature distribution of the compartment and the air intake rate were measured. The heat release rates of the fuel were calculated and the smoke temperature was used as a validation parameter. A scoring system was proposed to compare the results predicted by the three models. According to the author, CFAST does 'particularly well,' though there are some differences which can be attributed to the zone model approach.

A second series of experiments by Luo [54] indicate that the CFAST model (version 3.1) generally over predicts the upper layer temperature in the burn room because the two-zone assumption is likely to break down in the burn room. It was found that the room averaged temperatures obtained from CFAST were in 'good overall agreement' with the experimental results. The discrepancies 
can be attributed to the correction needed for thermocouple measurements. The CO concentration, however, was inconsistent. CFAST tended to overestimate $\mathrm{CO}$ concentration when the air handling system was in operation. This was seen due to inconsistencies in what is measured (point measurements) and predicted (global measurements).

\section{Sprinkler Activation}

A suppression algorithm [55] was incorporated into CFAST. Chow [56] evaluates the predictive capability for a sprinkler installed in an atrium roof. There were three main points being considered: the possibility of activating the sprinkler, thermal response, and water requirement. The zone model CFAST was used to analyze the possibility of activation of a sprinkler head. Results derived from CFAST were seen to be 'accurate, that is, providing good agreement with experimental measurements.'

\section{$\mathbf{t}^{2}$ Fires}

Matsuyama conducted a series of full-scale experiments [57] using $\mathrm{t}^{2}$ fires. Fire room and corridor smoke filling processes were measured. The size of the corridors and arrangements of smoke curtains were varied in several patterns. Comparisons were then made between the experimental results and those predicted by CFAST. The author concludes that while the model does a 'good job' of predicting experimental results, there are systematic differences which could be reduced with some revision to zone model formulation to include the impact of smoke curtains. 


\section{Chapter 5}

\section{Description of Experiments}

This chapter summarizes the range of experiments used in the current evaluation for the CFAST model. This study focused on the predicted results of the CFAST fire model and did not include an assessment of the user interface for the model. However, all input files used for the simulations were prepared using the CFAST graphical user interface (GUI) and reviewed for correctness prior to the simulations. The comparisons between the experiments and model predictions were characterized as semi-blind calculations, i.e., the modelers were given detailed descriptions of the test conditions, test geometry, and fire source, but did not modify model inputs from these given conditions to improve model predictions. As such, the comparisons in this report provide an assessment of the predictive capability of the model, but not an assessment of the ability of different modelers to develop appropriate model inputs.

\subsection{NBS Single Room Tests with Furniture}

These data describe a series of room fire tests using upholstered furniture items in a room of fixed size but with varying opening sizes and shapes [34] conducted by the National Bureau of Standards (NBS, former name of NIST). It was selected for its well characterized and realistic fuel sources in a simple single-room geometry. In addition, the wide variation in opening size should provide challenges for current zone fire models. Peak fire size was about 2.9 MW with a total room volume of $21 \mathrm{~m}^{3}$. A series of four single-room fire tests were conducted using upholstered furniture items for comparison with their free burning behavior, previously determined in a furniture calorimeter. The experiments were conducted in a single room enclosure; ventilation to the room was provided by window openings of varying sizes. The room was equipped with an instrumented exhaust collection system outside the window opening.

A second similar test series also utilized a single-room fire test with furniture as the fire source [58]. It expanded upon the first data set by adding the phenomenon of wall burning. Peak fire size was about $7 \mathrm{MW}$. The room size was similar to the first test series. Figure 5.1 illustrates the configuration for the two test series.

The test furniture included a $28.3 \mathrm{~kg}$ armchair or a similar $40.0 \mathrm{~kg}$ love seat for the first test series. Both were of conventional wood frame construction and used polyurethane foam padding, made to minimum California State flammability requirements, and polyolefin fabric. A single piece of test furniture and igniting wastebasket were the only combustibles in the test room. 

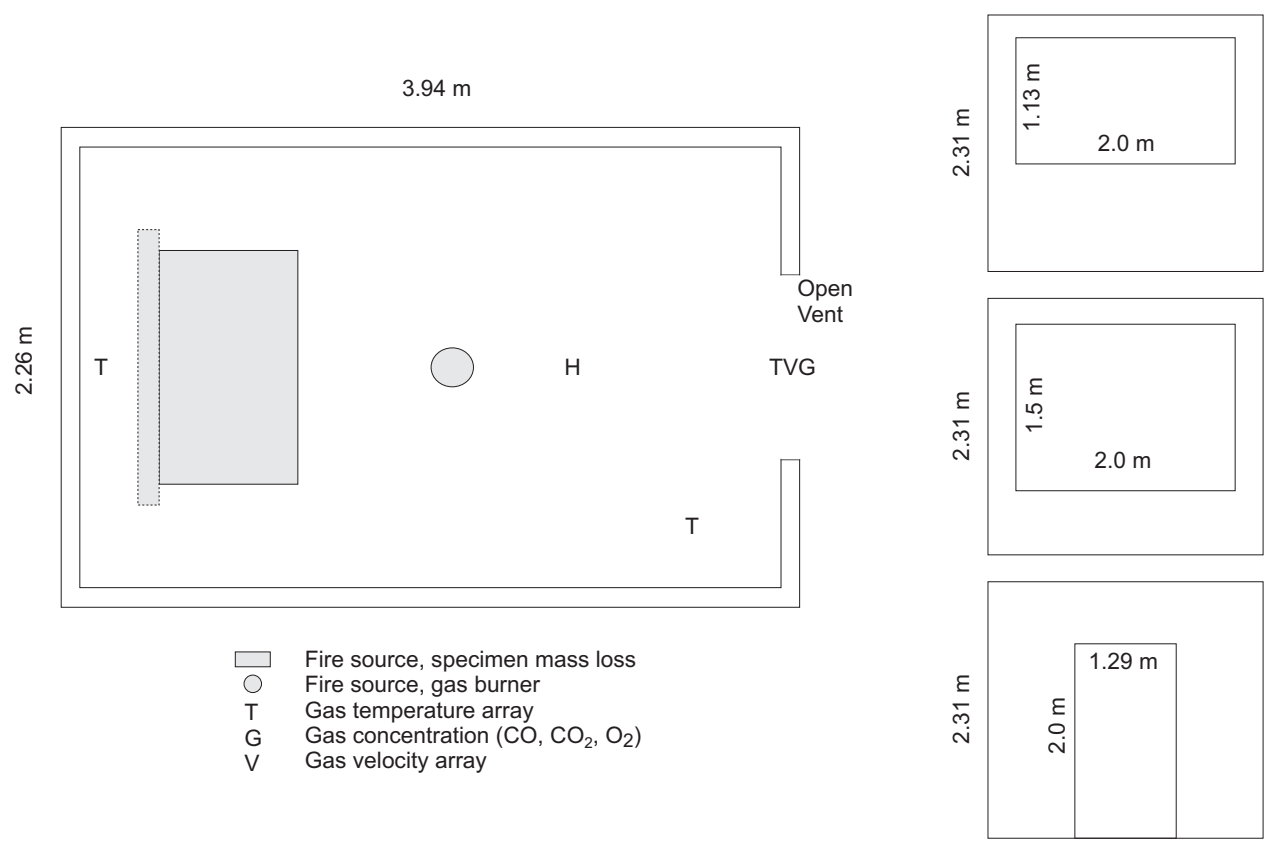

Figure 5.1: Plan and elevation view schematic of experimental room for NBS single room tests with furniture. Note dotted lines on burning specimen indicates vertical surface for wall burning experiments. Specimen and instrumentation placement are approximate.

For the second test series, room furnishings consisted of a $1.37 \mathrm{~m}$ wide $\times 1.91 \mathrm{~m}$ long $\mathrm{x} 0.53 \mathrm{~m}$ high double bed, a $2.39 \mathrm{~m} \mathrm{X} 0.89 \mathrm{~m}$ high headboard, and $0.51 \mathrm{~m}$ wide $\times 0.41 \mathrm{~m}$ deep x $0.63 \mathrm{~m}$ high night table. Both headboard and night table were fabricated from $12.7 \mathrm{~mm}$ thick plywood. The bedding was comprised of two pillows, two pillow cases, two sheets, and one blanket. The pillows had a polypropylene fabric with a polyester filling. The pillow cases and sheets were polyestercotton. The blanket was acrylic material. The bedding was left in a "slept in" condition which was duplicated to the degree possible in each test. In all of the tests, the fire was started with match flame ignition of a $0.34 \mathrm{~kg}$ ( $240 \mathrm{~mm}$ x $140 \mathrm{~mm}$ x $240 \mathrm{~mm}$ high) wastebasket, filled with $0.41 \mathrm{~kg}$ of trash, positioned adjacent to the bed.

\subsection{VTT Large Hall Tests}

The experiments are described in reference [59]. The series consisted three unique fire scenarios with replications for a total of 8 experiments. The experiments were undertaken to study the movement of smoke in a large hall with a sloped ceiling. The tests were conducted inside the VTT Fire Test Hall, with dimensions of $19 \mathrm{~m}$ high by $27 \mathrm{~m}$ long by $14 \mathrm{~m}$ wide. Figure 5.2 shows the important features of the test hall. Figure 5.3 shows detailed plan, side and perspective schematic diagrams of the experimental arrangement. Each test involved a single heptane pool fire, ranging from $2 \mathrm{MW}$ to $4 \mathrm{MW}$. Figure 5.4 is a photo of a $2 \mathrm{MW}$ fire. Four types of measurements were used in the present evaluation - the hot gas layer temperature and depth, average flame height and the plume temperature. Three vertical arrays of thermocouples, plus two thermocouples in the 


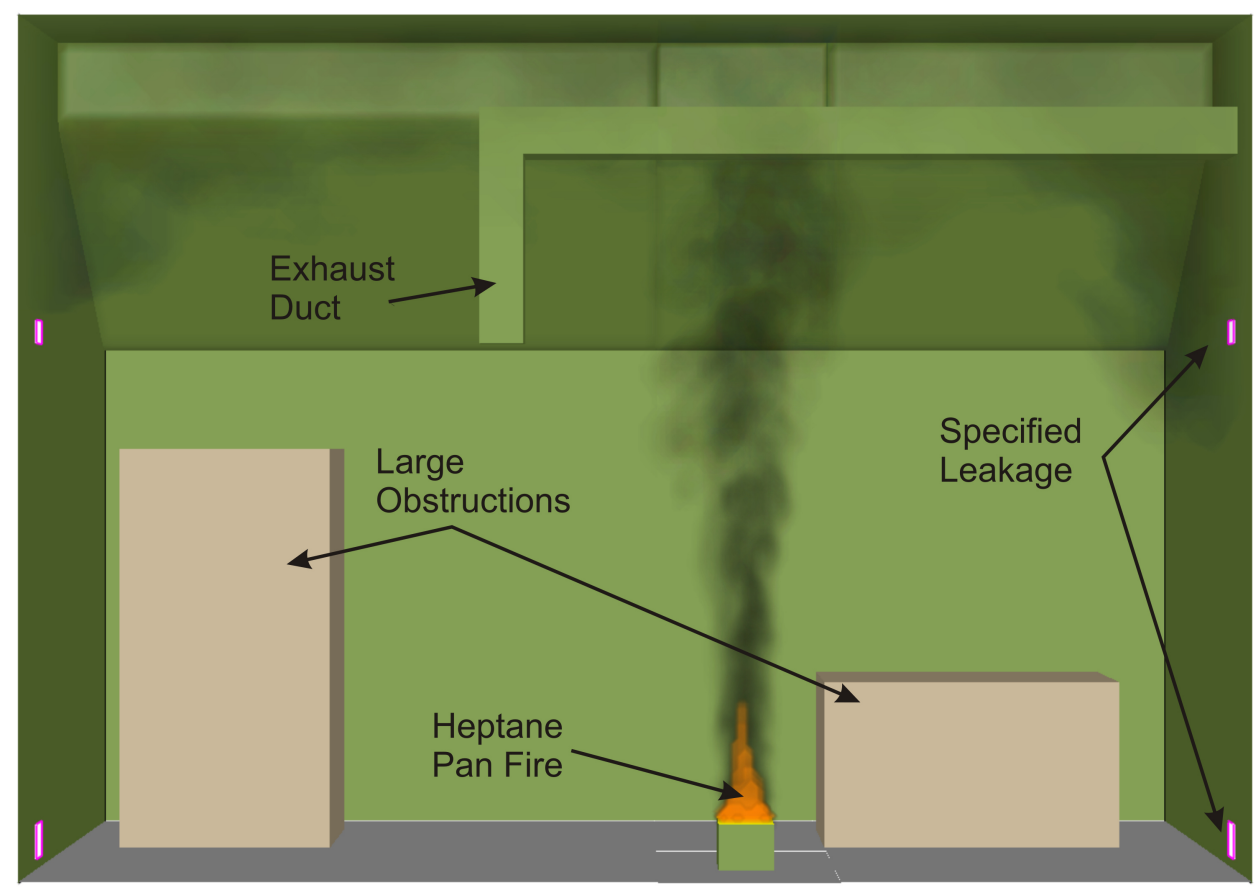

Figure 5.2: Cut-Away View of Case 2 of the VTT Large Hall Tests.

plume, were compared to model simulation results. The hot gas layer temperature and height were reduced from an average of the three thermocouple arrays using a standard algorithm. The ceiling jet temperature was not considered, because the ceiling in the test hall is not flat, and the model algorithm is not appropriate for these conditions.

The VTT test report lacks some information needed to model the experiments, so some information was based on private communications with the principal investigator, Simo Hostikka. Details used to conduct the model simulations is presented in reference [60], including information on the fire, the compartment, and the ventilation.

The walls and ceiling of the test hall consist of a $1 \mathrm{~mm}$ thick layer of sheet metal on top of a $5 \mathrm{~cm}$ layer of mineral wool. The floor was constructed of concrete. The report does not provide thermal properties of these materials. Thermophysical properties of the materials that were used in the simulations are given in table 5.1 .

Table 5.1: Thermophysical Properties for VTT Large Hall Tests

\begin{tabular}{|l|c|c|c|c|c|}
\hline Material & $\begin{array}{c}\text { Conductivity } \\
\mathrm{W} / \mathrm{m}^{\circ} \mathrm{C}\end{array}$ & $\begin{array}{c}\text { Specific Heat } \\
\mathrm{J} / \mathrm{kg}^{\circ} \mathrm{C}\end{array}$ & $\begin{array}{c}\text { Density } \\
\mathrm{kg} / \mathrm{m}^{3}\end{array}$ & $\begin{array}{c}\text { Thickness } \\
\mathrm{m}\end{array}$ & Emissivity \\
\hline \hline Steel ICFMP BE2 & 54 & 425 & 7850 & 0.001 & 0.95 \\
\hline Concrete ICFMP BE2 & 2 & 900 & 230 & 0.15 & 0.95 \\
\hline
\end{tabular}

In Cases 1 and 2, all doors were closed, and ventilation was restricted to leakage through the building envelope. Precise information on air infiltration during these tests is not available. The 


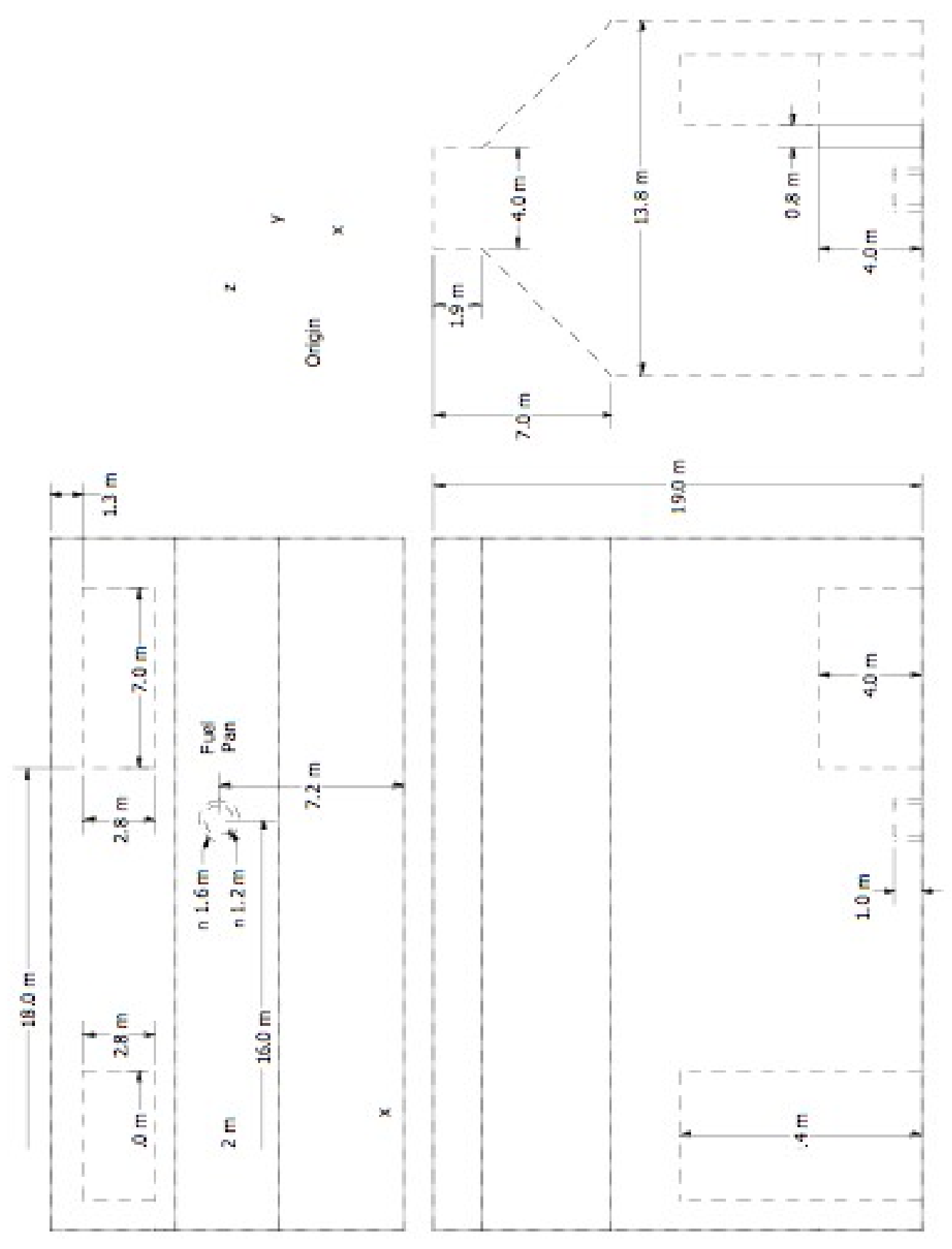

Figure 5.3: Plan, side and perspective schematic drawings of the experimental arrangement of the VTT large hall fire tests, including the fuel pan 


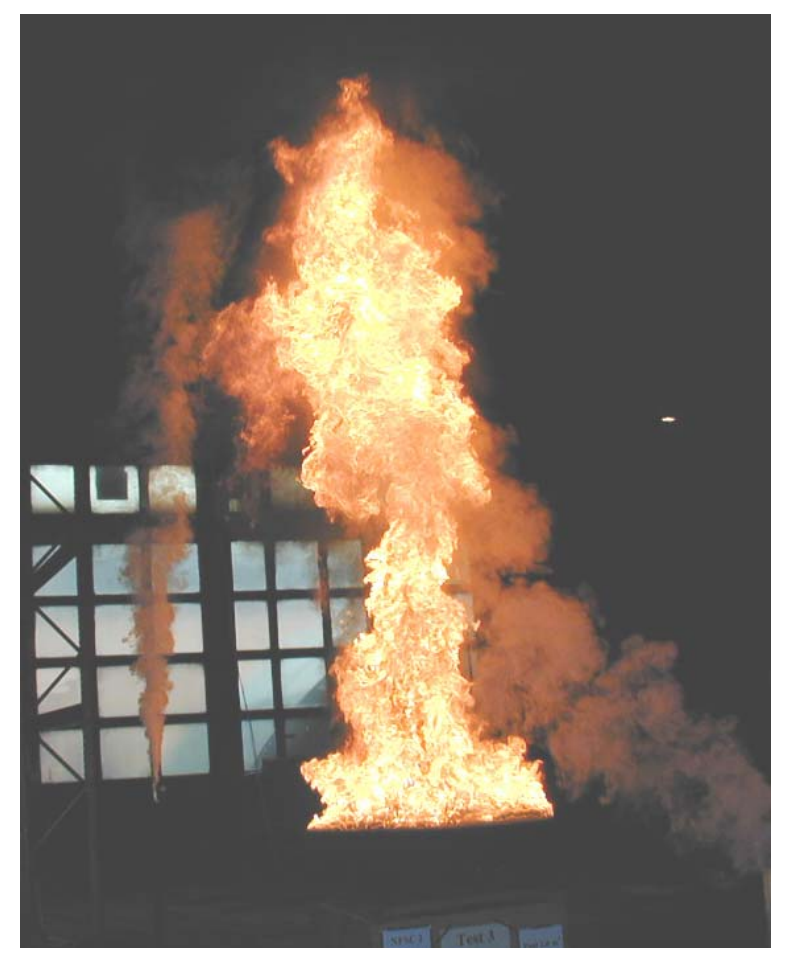

Figure 5.4: Photo of a 2 MW heptane fire during the VTT large hall tests. Photo provided by Simo Hostikka, VTT.

scientists who conducted the experiments recommend a leakage area of about $2 \mathrm{~m}^{2}$, distributed uniformly throughout the enclosure. By contrast, in Case 3, the doors located in each end wall (Doors 1 and 2, respectively) were open to the external ambient environment. These doors are each $0.8 \mathrm{~m}$ wide by $4 \mathrm{~m}$ high, and are located such that their centers are $9.3 \mathrm{~m}$ from the south wall. The test hall had a single mechanical exhaust duct, located in the roof space, running along the center of the building. This duct had a circular section with a diameter of $1 \mathrm{~m}$, and opened horizontally to the hall at a distance of $12 \mathrm{~m}$ from the floor and $10.5 \mathrm{~m}$ from the west wall. Mechanical exhaust ventilation was operational for Case 3, with a constant volume flow rate of $11 \mathrm{~m}^{3} / \mathrm{s} \mathrm{drawn}$ through the $1 \mathrm{~m}$ diameter exhaust duct.

Each test used a single fire source with its center located $16 \mathrm{~m}$ from the west wall and $7.4 \mathrm{~m}$ from the south wall. For all tests, the fuel was heptane in a circular steel pan that was partially filled with water. The pan had a diameter of $1.17 \mathrm{~m}$ for Case 1 and $1.6 \mathrm{~m}$ for Cases 2 and 3 . In each case, the fuel surface was $1 \mathrm{~m}$ above the floor. The trays were placed on load cells, and the HRR was calculated from the mass loss rate. For the three cases, the fuel mass loss rate was averaged from individual replicate tests. In the HRR estimation, the heat of combustion (taken as $44.6 \mathrm{~kJ} / \mathrm{g}$ ) and the combustion efficiency for $n$-heptane was used. In this report, a combustion efficiency of $0.85 \pm 0.12$ ( or $\pm 14 \%$ ) was used for the VTT pool fire tests [60]. Due to the relatively large value of the uncertainty associated with the combustion efficiency the uncertainty in HRR is dominated by the uncertainty in the combustion efficiency. Uncertainty in the mass loss rate measurement also contributed to the overall uncertainty, and the uncertainty in HRR was estimated as 15\% [60]. Figure 5.5 show the prescribed HRR as a function of time during Cases 1 to 3, respectively. The 
radiative fraction was assigned a value of 0.35 [60], similar to many smoky hydrocarbons [61]. The relative combined expanded $(2 \sigma)$ uncertainty in this parameter was assigned a value of $\pm 20 \%$, which is typical of uncertainty values reported in the literature for this parameter. Further details of the model inputs used for these simulations are included in reference [60].

\subsection{NIST/NRC Test Series}

These experiments, sponsored by the US NRC and conducted at NIST, consisted of 15 largescale experiments performed in June 2003. All 15 tests were included in the validation study. The experiments are documented in Ref. [62]. The fire sizes ranged from $350 \mathrm{~kW}$ to $2.2 \mathrm{MW}$ in a compartment with dimensions $21.7 \mathrm{~m}$ by $7.1 \mathrm{~m}$ by $3.8 \mathrm{~m}$ high, designed to represent a compartment in a nuclear power plant containing power and control cables. A photo of the fire seen through the compartment doorway is shown in figure 5.6. Figure 5.7 shows the important features of the test hall. Figure 5.8 shows detailed plan, side and perspective schematic diagrams of the experimental arrangement. The walls and ceiling were covered with two layers of marinate boards, each layer $0.0125 \mathrm{~m}$ thick. The floor was covered with one layer of gypsum board on top of a layer of plywood. Thermo-physical and optical properties of the marinate and other materials used in the compartment are given in reference [62]. The room had one door and a mechanical air injection and extraction system. Ventilation conditions, the fire size, and fire location were varied. Numerous measurements (approximately 350 per test) were made including gas and surface temperatures, heat fluxes and gas velocities. Detailed schematic diagrams of the experimental arrangement are shown in figure 5.8. Table 5.2 shows the experimental conditions for all 15 tests.

The compartment had a $2 \mathrm{~m}$ by $2 \mathrm{~m}$ door in the middle of the west wall. Some of the tests had a closed door and no mechanical ventilation, and in those tests the measured compartment leakage was an important consideration. Reference [62] reports leakage area based on measurements performed periodically during the test series. For the closed door tests, the leakage area used in the simulations was based on the last available measurement. It should be noted that the chronological order of the tests differed from the numerical order [62].

The mechanical ventilation and exhaust was used during some test, providing about 5 air changes per hour. The supply duct was positioned on the long wall, about $2 \mathrm{~m}$ off the floor. An exhaust duct of equal area to the supply duct was positioned on the opposite wall at a comparable location. The flow rates through the supply and exhaust ducts were measured in detail during breaks in the testing, in the absence of a fire. During the tests, the flows were monitored with single bi-directional probes during the tests themselves.

A single nozzle was used to spray liquid hydrocarbon fuels onto a $1 \mathrm{~m}$ by $2 \mathrm{~m}$ fire pan that was about $0.02 \mathrm{~m}$ deep. The test plan originally called for the use of two nozzles to provide the fuel spray. Experimental observation suggested that the fire was more steady with the use of a single nozzle. In addition, it was observed that the actual extent of the liquid pool was well-approximated by a $1 \mathrm{~m}$ circle in the center of the pan. For safety reasons, the fuel flow was terminated when the lower-layer oxygen concentration dropped to approximately $15 \%$ by volume. The fuel used in 14 of the tests was heptane, while toluene was used for one test. The HRR was determined using oxygen consumption calorimetry (figure 5.9 shows a sample heat release rate for one of the tests in the series). The recommended uncertainty values for HRR were $17 \%$ for all of the tests. The radiative fraction was measured in an independent study for the same fuels using the same spray 

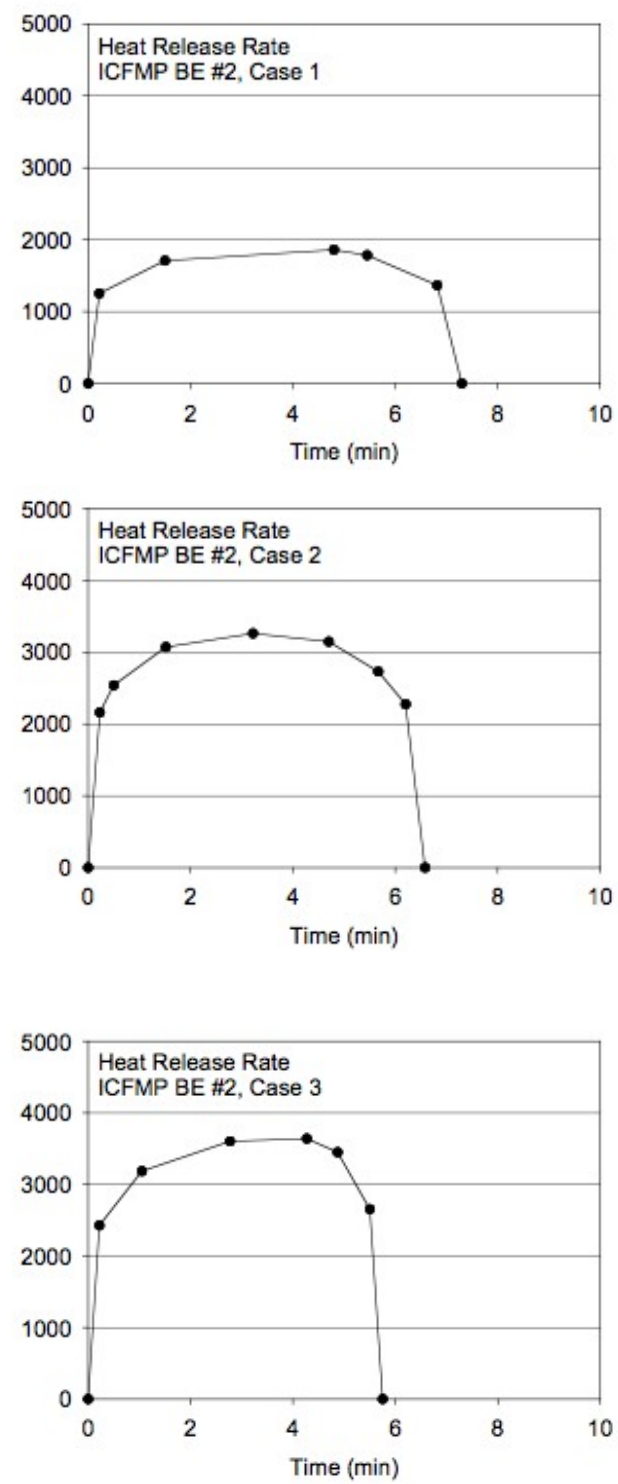

Figure 5.5: Prescribed Heat Release Rate as a Function of Time for VTT Large Hall Tests. 


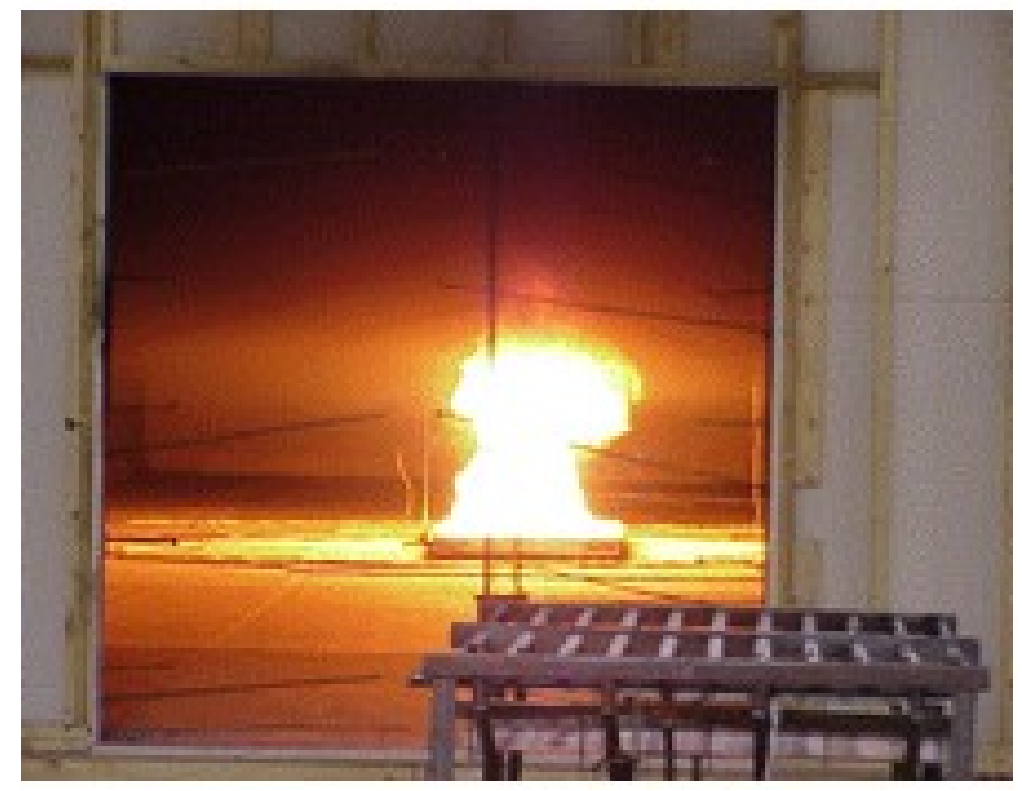

Figure 5.6: Photograph of a $1 \mathrm{MW}$ heptane fire seen through the open doorway. Photo provided by Anthony Hamins, NIST.

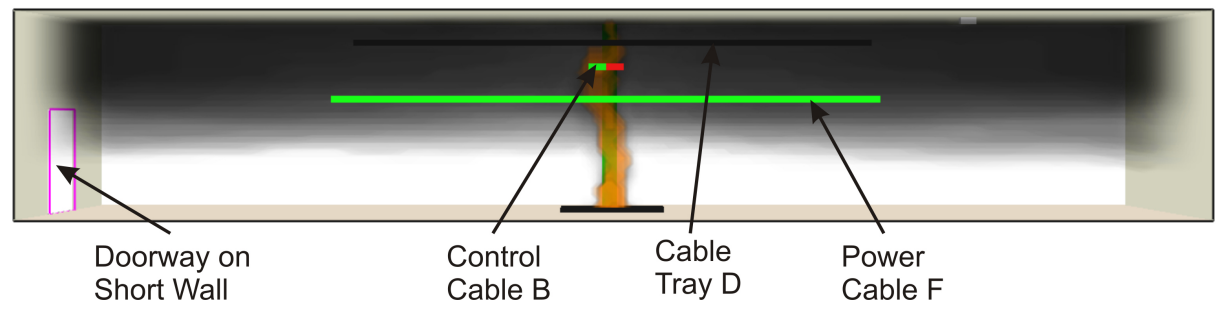

Figure 5.7: Cross-section View of the NIST NRC Test Configuration. 

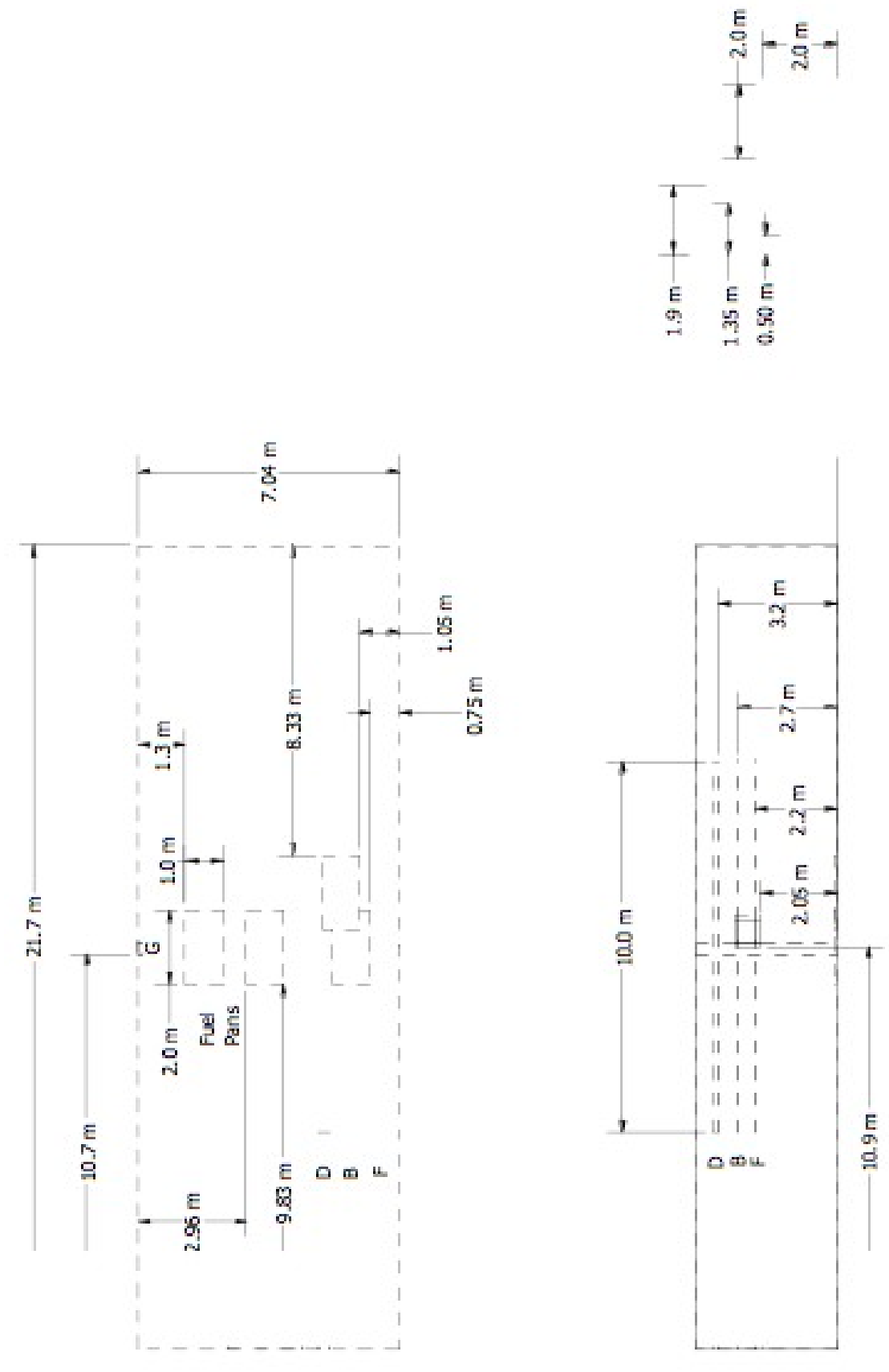

Figure 5.8: Plan, side and perspective schematic drawings of the NIST NRC experimental arrangement. The fuel pan and cables B, D, F, and G (dotted lines) are also shown. 
Table 5.2: Test Matrix and Experimental Conditions for NIST NRC Tests

\begin{tabular}{|c|c|c|c|c|c|}
\hline Test & $\begin{array}{l}\text { Nominal Peak } \\
\dot{Q}(\mathrm{MW})\end{array}$ & $\begin{array}{l}\text { Cable } \\
\text { Type }\end{array}$ & Fuel; Burner Location & Door & $\begin{array}{l}\text { Mechanical } \\
\text { Ventilation }\end{array}$ \\
\hline 1 & 0.35 & $\mathrm{XPE}^{\mathrm{a}}$ & Heptane; Center & Closed & Off \\
\hline 2 & 1 & XPE & Heptane; Center & Closed & Off \\
\hline 3 & 1 & XPE & Heptane; Center & Open & Off \\
\hline 4 & 1 & XPE & Heptane; Center & Closed & On \\
\hline 5 & 1 & XPE & Heptane; Center & Open & On \\
\hline 6 & \multicolumn{5}{|c|}{ Not Conducted } \\
\hline 7 & 0.35 & $\mathrm{PVC}^{\mathrm{b}}$ & Heptane; Center & Closed & Off \\
\hline 8 & 1 & XPE & Heptane; Center & Closed & Off \\
\hline 9 & 1 & XPE & Heptane; Center & Open & Off \\
\hline 10 & 1 & PVC & Heptane; Center & Closed & On \\
\hline 11 & \multicolumn{5}{|c|}{ Not Conducted } \\
\hline 12 & \multicolumn{5}{|c|}{ Not Conducted } \\
\hline 13 & 2 & XPE & Heptane; Center & Closed & Off \\
\hline 14 & 1 & XPE & $\begin{array}{l}\text { Heptane; } 1.8 \mathrm{~m} \text { from } \mathrm{N} \text { wall } \\
\text { on } \mathrm{E}-\mathrm{W} \text { centerline }\end{array}$ & Open & Off \\
\hline 15 & 1 & PVC & $\begin{array}{l}\text { Heptane; } 1.25 \mathrm{~m} \text { from } \mathrm{S} \text { wall } \\
\text { on } \mathrm{E}-\mathrm{W} \text { centerline }\end{array}$ & Open & Off \\
\hline 16 & 2 & PVC & Heptane; Center & Closed & On \\
\hline 17 & 1 & PVC & Toluene; Center & Closed & Off \\
\hline 18 & 1 & XPE & $\begin{array}{l}\text { Heptane; } 1.55 \mathrm{~m} \text { from } \mathrm{S} \text { wall } \\
1.50 \mathrm{~m} \mathrm{E} \text { of centerline }\end{array}$ & Open & Off \\
\hline
\end{tabular}

a - XPE cable has crosslinked polyethylene jacket insulation

b - PVC cable has a polyvinylchloride jacket insulation 


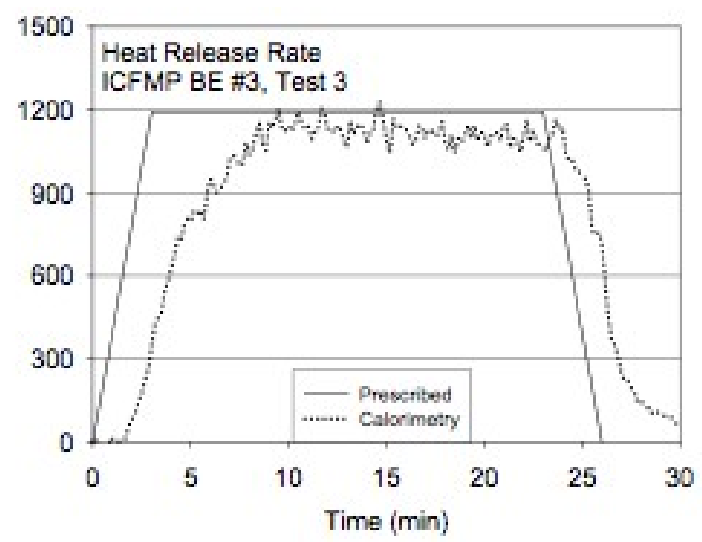

Figure 5.9: Measured and prescribed heat release rate as a function of time during Test 3 of the NIST NRC test series

burner as used in the test series [63]. The value of the radiative fraction and its uncertainty were reported as $0.44 \pm 16 \%$ and $0.40 \pm 23 \%$ for heptane and toluene, respectively. Further details of the model inputs used for these simulations are included in reference [60].

\subsection{FM/SNL Test Series}

The Factory Mutual and Sandia National Laboratories (FM/SNL) Test Series was a series of 25 fire tests conducted in 1985 for the NRC by Factory Mutual Research Corporation (FMRC), under the direction of Sandia National Laboratories (SNL). The primary purpose of these tests was to provide data with which to validate computer models for various types of NPP compartments. The experiments were conducted in an enclosure measuring $18 \mathrm{~m} \times 12 \mathrm{~m} \times 6 \mathrm{~m}$, constructed at the FMRC fire test facility in Rhode Island. Figure 5.10 shows detailed schematic drawings of the compartment from various perspectives. The FM/SNL test series is described in detail, including the types and locations of measurement devices, as well as some results in References [64, 65]. Six of the experiments were conducted with a full-scale control room mock-up in place. Parameters varied during the experiments included fire intensity, enclosure ventilation rate, and fire location. The current guide uses data from nineteen experiments (Tests 1-17, 21, and 22). In these tests, propylene gas burners, heptane pools, and methanol pools were used as fire sources. Table 5.3 lists the test parameters.

The following information was provided by the test director, Steve Nowlen of Sandia National Laboratory. In particular, Tests 4, 5, and 21 were given extra attention.

Heat Release Rate: The HRR was determined using oxygen consumption calorimetry in the exhaust stack with a correction applied for the carbon dioxide in the upper layer of the compartment. The uncertainty of the fuel mass flow was not documented. Several tests selected for this study had the same target peak heat release rate of $516 \mathrm{~kW}$ following a $4 \mathrm{~min}$ "tsquared" growth profile. The test report contains time histories of the measured HRR, for which the average, sustained HRR following the ramp up for Tests 4, 5, and 21 have been estimated as $510 \mathrm{~kW}, 480 \mathrm{~kW}$, and $470 \mathrm{~kW}$, respectively. Once reached, the peak HRR was 
maintained essentially constant during a steady-burn period of $6 \min$ in Tests 4 and 5, and 16 min in Test 21. Note that in Test 21, Nowlen reports a "significant" loss of effluent from the exhaust hood that could lead to an under-estimate of the HRR towards the end of the experiment.

Radiative Fraction: The radiative fraction was not measured during the experiment, but in this study it is assumed to equal 0.35 , which is typical for a smoky hydrocarbons. It was further assumed that the radiative fraction was about the same in Test 21 as the other tests, as fuel burning must have occurred outside of the electrical cabinet in which the burner was placed.

Measurements: Four types of measurements were conducted during the FM/SNL test series that are used in the current model evaluation study, including the HGL temperature and depth, and the ceiling jet and plume temperatures. Aspirated thermocouples (TCs) were used to make all of the temperature measurements. Generally, aspirated TC measurements are preferable to bare-bead TC measurements, as systematic radiative exchange measurement error is reduced.

HGL Depth and Temperature: Data from all of the vertical TC trees were used when reducing the HGL height and temperature. For the majority of the tests, Sectors 1, 2, and 3 were used, all weighted evenly. For Tests 21 and 22, Sectors 1 and 3 were used, evenly weighted. Sector 2 was partially within the fire plume.

All of the tests involved forced ventilation to simulate typical nuclear power plant installation practices.

\section{5 iBMB Compartment Tests}

A series of small compartment kerosene pool fire experiments, conducted at the Institut fr Baustoffe, Massivbau und Brandschutz (iBMB) of Braunschweig University of Technology in Germany in 2004 [66]. The results from Test 1 were considered here. These experiments involved relatively large fires in a relatively small $(3.6 \mathrm{~m}$ x $3.6 \mathrm{~m}$ x $5.7 \mathrm{~m})$ concrete enclosure. Figure 5.12 shows plan, side and perspective schematic drawings of the experimental arrangement, including the location of the fuel pan, which was located at the center of the compartment.

Only a portion of Test 1 was selected for consideration in the present study, because a significant amount of data was lost in Test 2 , and the measured $\dot{Q}$ during Test 3 exhibited significant amounts of fluctuation. Five types of measurements that were conducted during the test series were used in the model evaluation reported here. These included the HGL temperature and depth, the temperature of targets and compartment surfaces, and heat flux. In addition, figure 5.13 shows the heat release rate for Test 1 , which was estimated from a mass loss rate measurement. In this calculation, the heat of combustion and the combustion efficiency were taken as $42.8 \mathrm{MJ} / \mathrm{kg}$ and 1 , respectively, as suggested by Refs. [60,67]. There were several reported difficulties in measuring the mass loss rate, including data loss due to an instrument malfunction and significant fluctuations in the measured mass loss rate. Due to these measurement issues and because the combustion efficiency was not well-characterized, the $\dot{Q}$ uncertainty was assigned a relatively large expanded uncertainty of $\pm 25 \%$ [60]. 

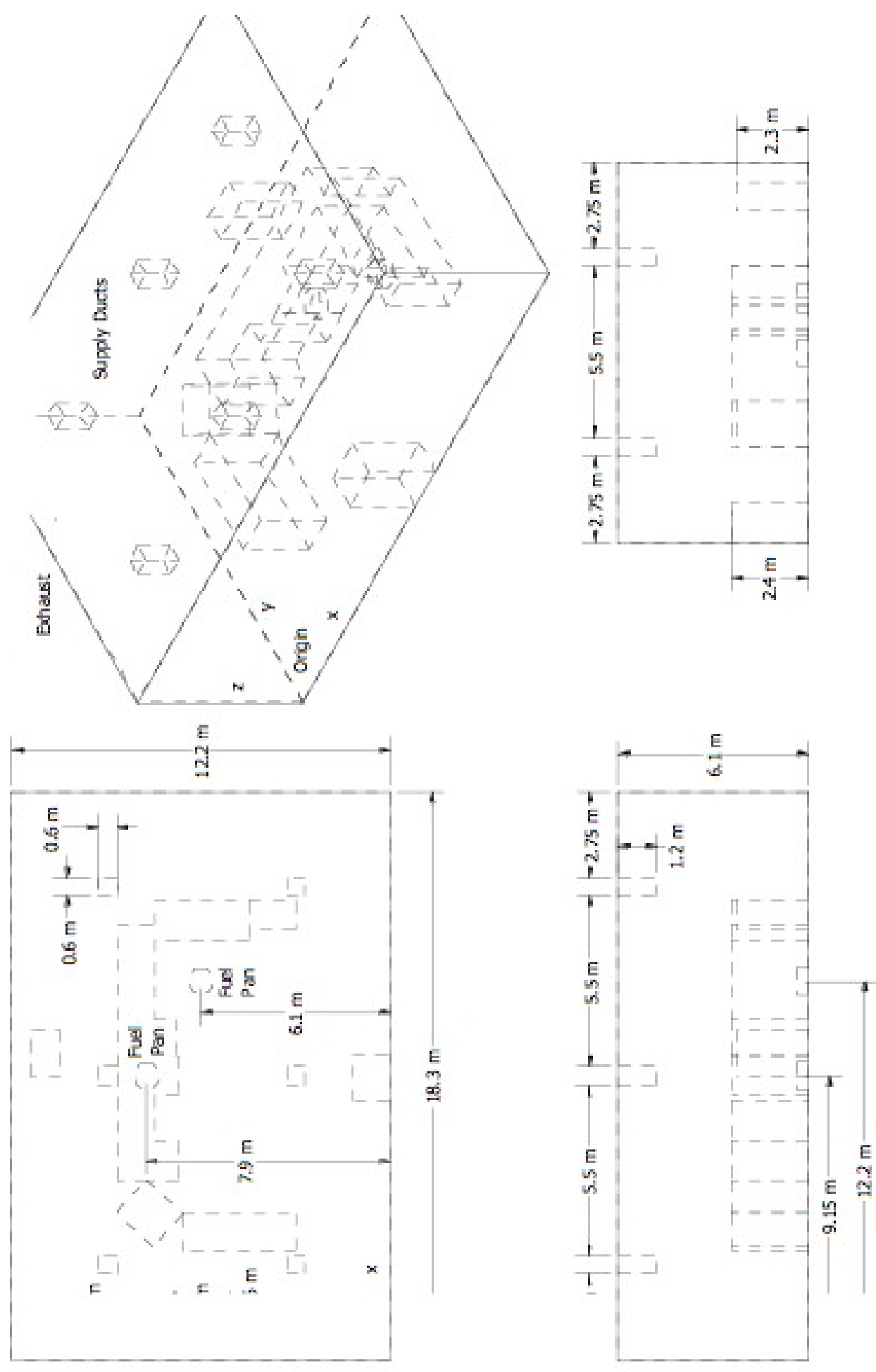

Figure 5.10: Detailed plan, side, and perspective schematic drawings of the FM/SNL experimental arrangement, including the supply and exhaust ducts, and the fuel pan. 
Table 5.3: Summary of FM/SNL Experiments.

\begin{tabular}{|c|c|c|c|c|c|c|}
\hline $\begin{array}{c}\text { Test } \\
\text { No. }\end{array}$ & $\begin{array}{c}\text { Fuel } \\
\text { Type }\end{array}$ & $\begin{array}{c}\text { Nominal Peak } \\
\text { HRR }(\mathrm{kW})\end{array}$ & $\begin{array}{c}\text { Fire } \\
\text { Position }\end{array}$ & $\begin{array}{c}\text { Ventilation } \\
\text { Rate }(\mathrm{ach})\end{array}$ & $\begin{array}{c}\text { Room } \\
\text { Configuration }\end{array}$ & $\begin{array}{c}\text { Used in } \\
\text { Guide? }\end{array}$ \\
\hline \hline 1 & Propylene Burner & 516 & Center & 10 & Empty & Yes \\
\hline 2 & Propylene Burner & 516 & Center & 10 & Empty & Yes \\
\hline 3 & Propylene Burner & 2000 & Center & 10 & Empty & Yes \\
\hline 4 & Propylene Burner & 516 & Center & 1 & Empty & Yes \\
\hline 5 & Propylene Burner & 516 & Center & 10 & Empty & Yes \\
\hline 6 & Heptane Pool & 500 & Wall & 1 & Empty & Yes \\
\hline 7 & Propylene Burner & 516 & Center & 1 & Empty & Yes \\
\hline 8 & Propylene Burner & 1000 & Center & 1 & Empty & Yes \\
\hline 9 & Propylene Burner & 1000 & Center & 8 & Empty & Yes \\
\hline 10 & Heptane Pool & 1000 & Wall & 4.4 & Empty & Yes \\
\hline 11 & Methanol Pool & 500 & Wall & 4.4 & Empty & Yes \\
\hline 12 & Heptane Pool & 2000 & Wall & 4.4 & Empty & Yes \\
\hline 13 & Heptane Pool & 2000 & Wall & 8 & Empty & Yes \\
\hline 14 & Methanol Pool & 500 & Wall & 1 & Empty & Yes \\
\hline 15 & Heptane Pool & 1000 & Wall & 1 & Empty & Yes \\
\hline 16 & Heptane Pool & 500 & Corner & 1 & Empty & Yes \\
\hline 17 & Heptane Pool & 500 & Corner & 10 & Empty & No \\
\hline 18 & PMMA Slab & 1000 & Wall & 1 & Empty & No \\
\hline 19 & Heptane Pool & 1000 & Center & 1 & Furnished & No \\
\hline 20 & Heptane Pool & 1000 & Corner & 8 & Furnished & Yes \\
\hline 21 & Propylene Burner & 500 & Cabinet & 1 & Furnished & Yes \\
\hline 22 & Propylene Burner & 1000 & Cabinet & 1 & Furnished & No \\
\hline 23 & Qualified Cable & N/A & Cabinet & 1 & Furnished & No \\
\hline 24 & Unqualified Cable & N/A & Cabinet & 1 & Furnished & No \\
\hline 25 & Unqualified Cable & N/A & Cabinet & 8 & Furnished & No \\
\hline
\end{tabular}

A second series of fire experiments in 2004, conducted under the International Collaborative Fire Model Project (ICFMP) involved realistically routed cable trays inside the same concrete enclosure at iBMB [67]. The compartment was configured slightly differently with a ceiling height of $5.6 \mathrm{~m}$. A schematic diagram from plan, side, and perspective views of the experimental arrangement is shown in figure 5.14. Six types of measurements conducted during the test series were used in the evaluation conducted here, including the HGL temperature and depth, oxygen gas concentration, the temperature of targets and compartment surfaces, and heat flux.

The results of four tests were available, of which one has been used in the current study. These tests were conducted primarily for the evaluation of cable ignition and flame spread. The results were erratic, and no replicate experiments were performed. Given the primitive nature of the ignition and spread algorithms within the models, it was decided that only a qualitative analysis would be possible with the data from three of the four experiments. However, in one experiment, 


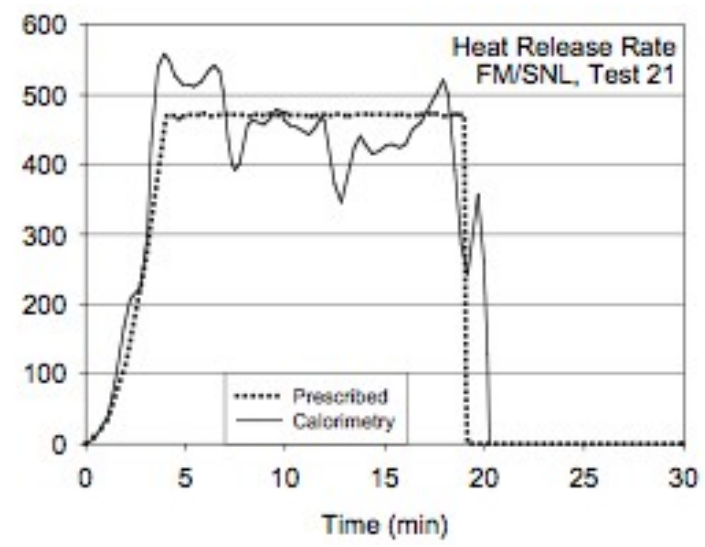

Figure 5.11: Prescribed (dotted line) and measured (solid line) heat release rate as a function of time during Test 21 of the FM/SNL test series

the first 20 minutes involved a fairly well-characterized ethanol pool fire burning on the opposite side of the compartment from the cable tray. This part of the experiment has been used as part of the model evaluation.

The first part of the test consisted of preheating the cable trays in the room with a $1 \mathrm{~m}^{2}$ round pan on the floor filled with ethanol (ethyl alcohol) used as the preheating source. At 30 min, a propane gas burner was used as the fire source this was not considered because only the first 20 min of the test was used in this validation study. Exhaust products were collected in an exhaust duct and the $\dot{Q}$ was measured using the oxygen calorimetry. For the purpose of this study, the measured $\dot{Q}$ was used as direct input to the various fire models. The first $20 \mathrm{~min}$ of data were used for the model evaluation. After $20 \mathrm{~min}$, the $\dot{Q}$ became relatively noisy. Figure 5.13 shows the HRR. The relative combined expanded uncertainty was assigned a value of $\pm 15 \%$, consistent with typical values of this parameter [60].

\subsection{NBS Multi-Compartment Test Series}

The National Bureau of Standards (NBS, former name of NIST) Multi-Compartment Test Series consisted of 45 fire tests representing 9 different sets of conditions were conducted in a three-room suite. The experiments were conducted in 1985 and are described in detail in reference [68]. The suite consisted of two relatively small rooms, connected via a relatively long corridor. Total volume of the structure was approximately $100 \mathrm{~m}^{2}$. The fire source, a gas burner, was located against the rear wall of one of the small compartments as seen in Figure 5.15 for a $100 \mathrm{~kW}$ fire. Figures 5.16 and 5.17 presents the experimental arrangement in the form of plan, side and perspective schematic drawings of the compartments. Fire tests of $100 \mathrm{~kW}, 300 \mathrm{~kW}$ and $500 \mathrm{~kW}$ were conducted. For the current study, three $100 \mathrm{~kW}$ fire experiments have been used, including Test 100A from Set 1, Test $100 \mathrm{O}$ from Set 2, and Test 100Z from Set 4. For the NBS Multi-room series, Tests 100A, $100 \mathrm{O}$ and $100 \mathrm{Z}$ were selected for study, because they were constructively used in a previous validation study [ [69], and because these tests had the steadiest values of measured heat release rate during the steady burning period. The selected data are also available in Reference [69]. Reference [60] 

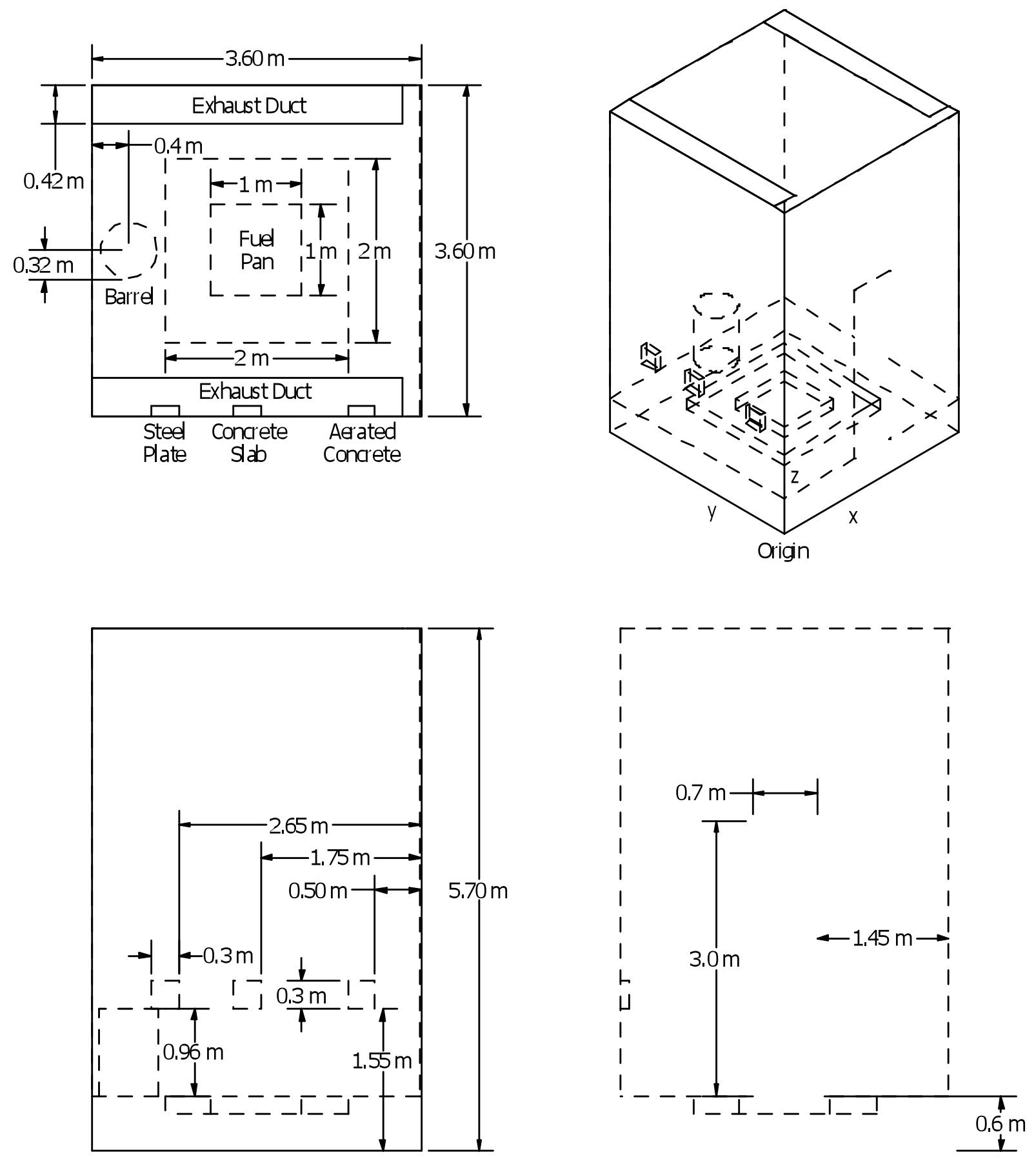

Figure 5.12: Detailed plan, side, and perspective schematic drawings of the iBMB pool fire experimental arrangement. 


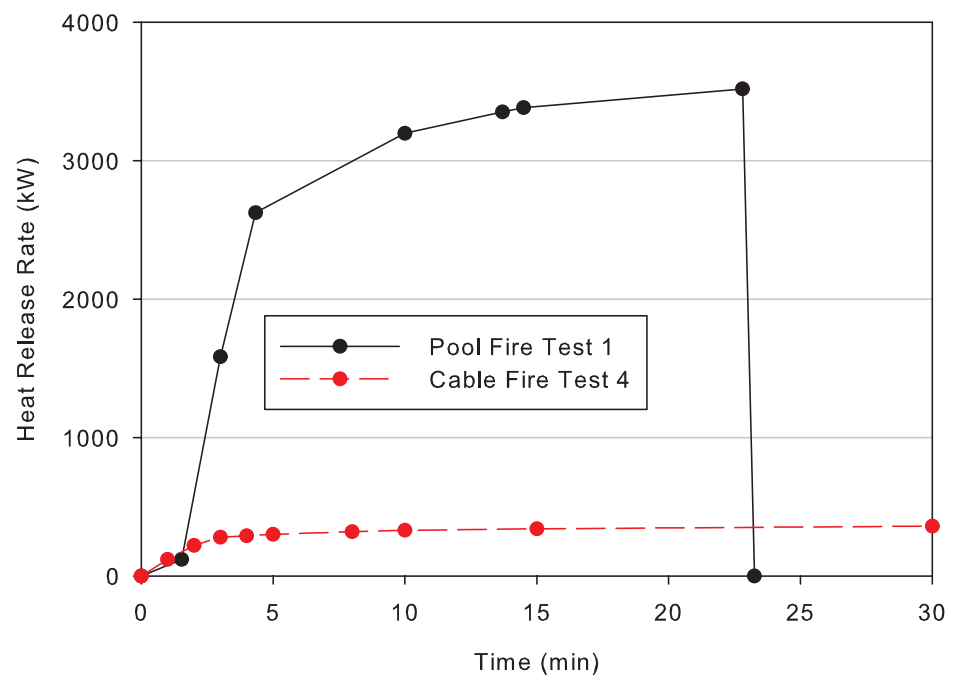

Figure 5.13: Estimated heat release rate for the iBMB fire experiments.

provides information used as model input for simulation of the NBS tests, including information on the compartment, the fire, the ventilation, and ambient conditions. The data in the NBS data set was acquired every $10 \mathrm{~s}$ with a $6 \mathrm{~s}$ time-average. This time-averaging interval was somewhat smaller than all of the other experimental series, which were time-averaged over a $10 \mathrm{~s}$ interval.

Figures 5.18 show the experimentally measured $\dot{Q}$ as a function of time during Tests $100 \mathrm{~A}$ and 100Z, respectively, of the NBS multi-room test series, typically averaging about $100 \mathrm{~kW}$. In these two tests, for which the door was open, the $\dot{Q}$ during the steady burning period measured via oxygen consumption calorimetry was about $110 \mathrm{~kW} \pm 17 \mathrm{~kW}( \pm 15 \%)$ [60]. The combined relative expanded $(2 \sigma)$ uncertainty in the calorimetric $\dot{Q}$ is assigned a value of $\pm 15 \%$, consistent with the replicate measurements made during the experimental series and the uncertainty typical of oxygen consumption calorimetry [60]. This value is also consistent with the measurement variation evident in the figures. It was assumed that the closed door test (Test 1000) had the same $\dot{Q}$ as the open door tests [60].

The specified or prescribed $\dot{Q}$ is also shown in the figures. The mass flow of the fuel (natural gas in Test 100A, or natural gas mixed with acetylene in Tests 1000 and 100Z) was not metered; rather, the effluent was captured in a hood mounted above the open door in the corridor and the was measured using oxygen consumption calorimetry. The manner by which the fuel flow was controlled is not documented. In Test 100A, candles were used to increase smoke in the upper layer to promote visualization. In Tests 1000 and 100Z, acetylene was used (about $20 \%$ by volume) to produce smoke. In those tests, the flow of natural gas and acetylene were adjusted to obtain approximately the same $\dot{Q}$ as in Test 100A. The addition of acetylene increased the radiative fraction of the fire.

For practical reasons, piped natural gas supplied by large utility companies is often used in fire experiments. While its composition may vary from day to day, there is little change expected in the value of the radiative fraction [60]. As mentioned above, natural gas was used as the fuel in Test 100A. In Tests 1000 and 100Z, acetylene was added to the natural gas to increase the smoke yield, 

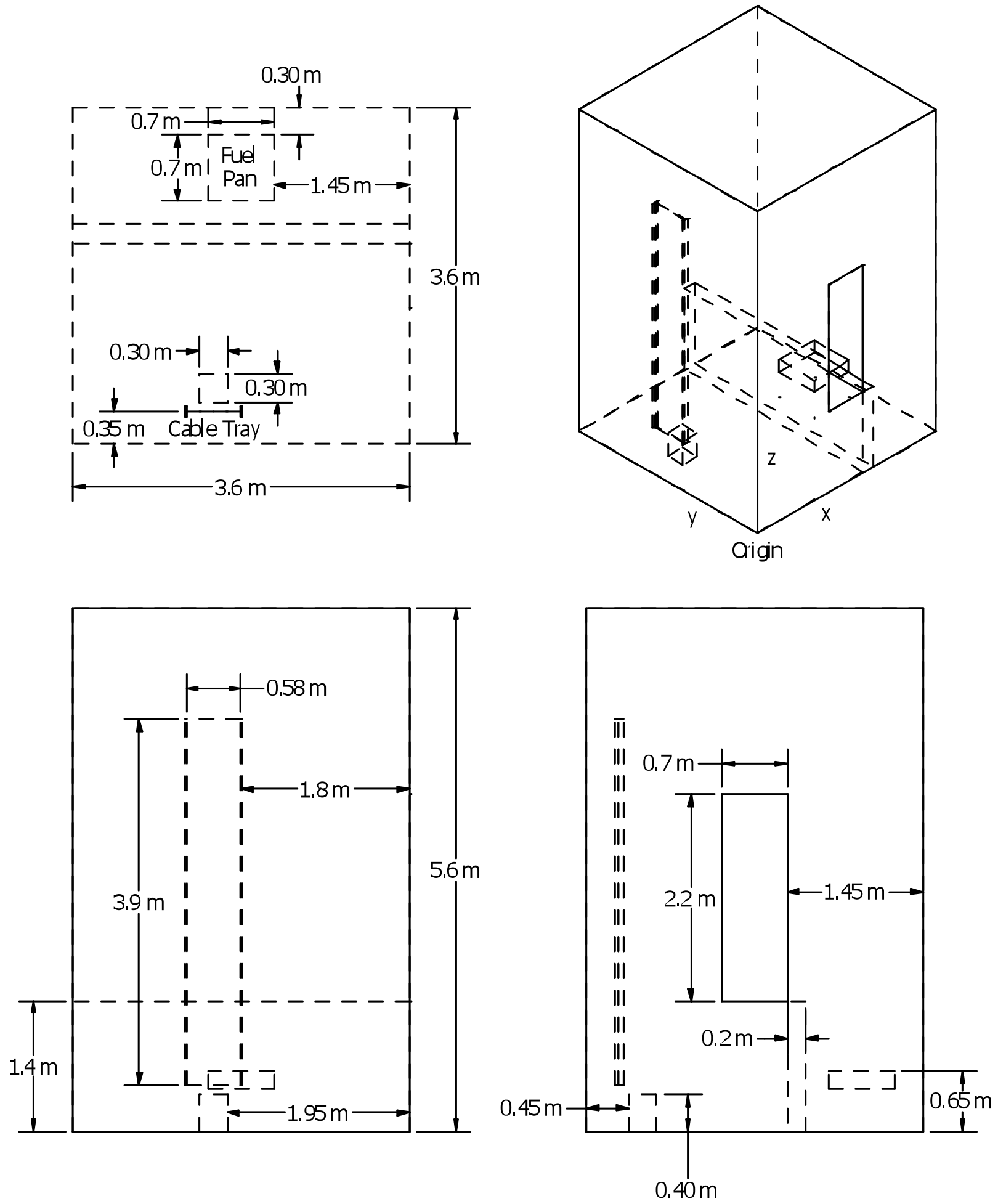

Figure 5.14: Detailed plan, side, and perspective schematic drawings of the iBMB cable fire experimental arrangement. 


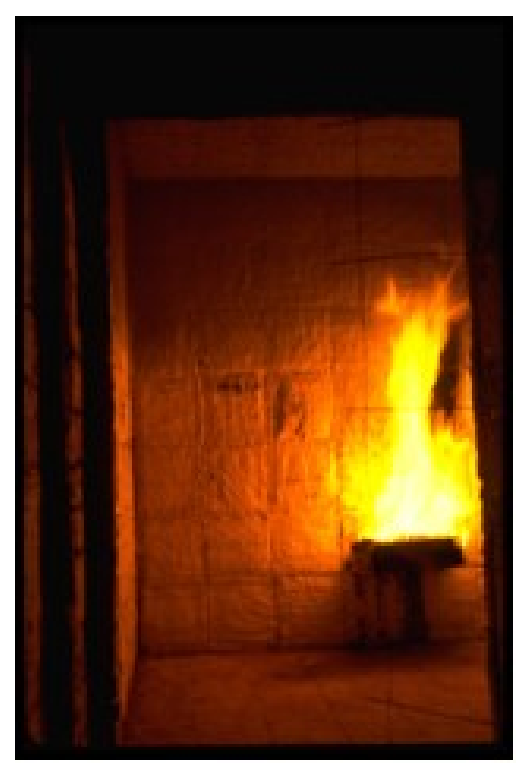

Figure 5.15: Photo of a $100 \mathrm{~kW}$ fire with the burner located against the rear wall of one of the small compartments in the NBS Multi-Compartment test Series.

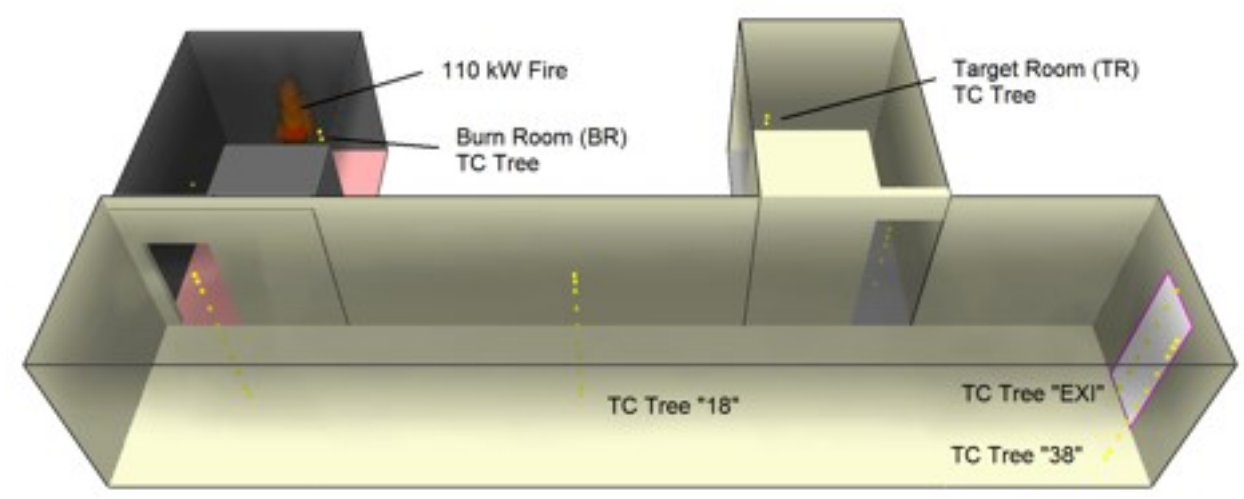

Figure 5.16: Overview of the NBS Test Configuration. 

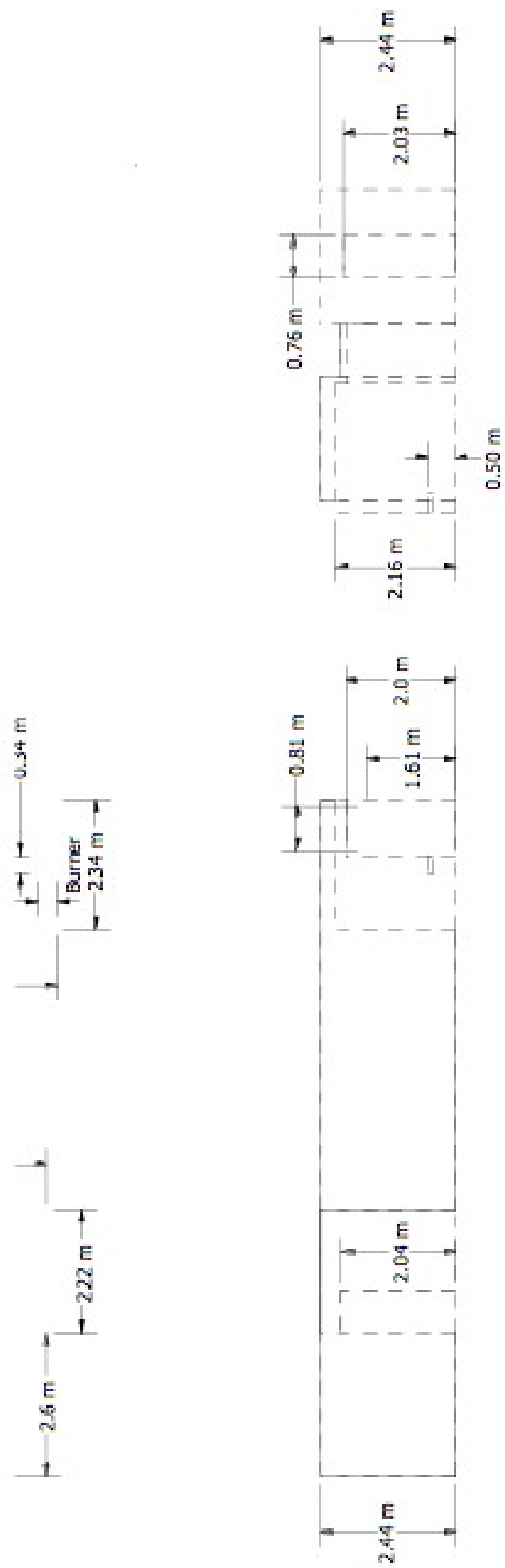

Figure 5.17: Plan, side and perspective schematic drawings of the NBS experimental arrangement, including the burner. 

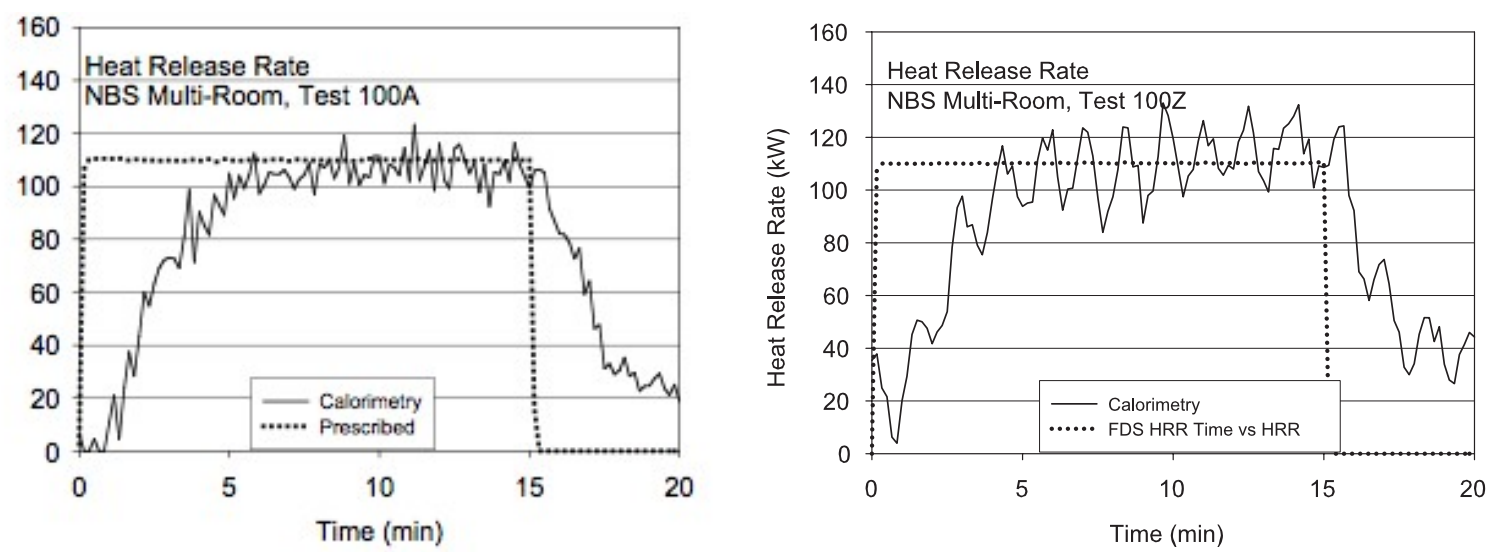

Figure 5.18: Prescribed and measured heat release rate as a function of time during Tests 100A and $100 Z$ of the NBS multi-room test series.

and as a consequence, the radiative fraction increased. The radiative fraction of natural gas has been studied previously, whereas the radiative fraction of the acetylene/natural gas mixture has not been studied. The radiative fraction for the natural gas fire was assigned a value of 0.20 , whereas a value of 0.30 was assigned for the natural gas/acetylene fires [60, 61].

The relative combined expanded $(2 \sigma)$ uncertainty in this parameter was assigned a value of $\pm 20 \%$ in Test $100 \mathrm{~A}$ and $\pm 30 \%$ in $100 \mathrm{O}$ and $100 \mathrm{Z}$. The $20 \%$ expanded deviation value is consistent with typical values of the deviation reported in the literature for the measured radiative fraction. The $100 \mathrm{O}$ and $100 \mathrm{Z}$ tests had a $50 \%$ larger value assigned, because the effect on the radiative fraction of adding acetylene to the natural gas was not measured [60].

Measurements made during the NBS test series included gas and surface temperature, pressure, smoke and major gas species concentration, and doorway gas velocity. Only two types of measurements conducted during the NBS test series were used in the evaluation considered here, because there was less confidence in the other measurements. The measurements considered here were the HGL temperature and depth, in which bare bead thermocouples were used to make these measurements. Single point measurements of temperature within the burn room were not used in the evaluation of plume or ceiling jet algorithms. This is because, in neither instance, was the geometry consistent with the assumptions used in the model algorithms of plumes or jets. Specifically, the burner was mounted against a wall, and the room width to height ratio was less than that assumed by the various ceiling jet correlations.

\subsection{FM Four Room Including Corridor Test Series}

This data set describes a series of tests conducted in a multiple room configuration with more complex gas burner fires than the previous data set. This study [70] was included because, in many ways, it is similar to the smoke movement study performed at NBS [68], and permits comparisons between two different laboratories. In addition, it expands upon that data set by providing larger a time-varying gas burner fires in a room-corridor configuration. Fire size was about up to $1 \mathrm{MW}$ with a total volume of $200 \mathrm{~m}^{3}$. 


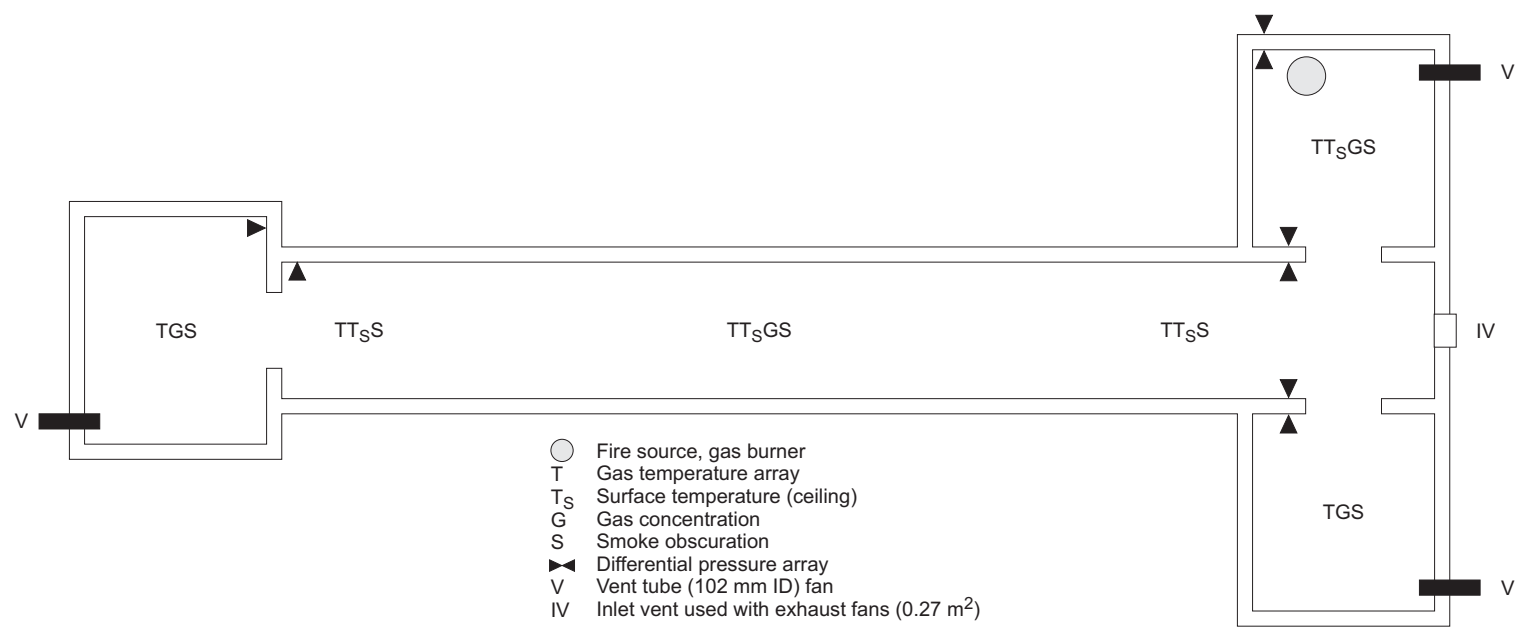

Figure 5.19: Overview of the Factory Mutual Four Room test series.

This study was performed to collect data allowing for variations in fire source, ventilation, and geometry in a multi-compartment structure, especially for situations with closed doors. This test program was carried out at Factory Mutual Research Corporation (FMRC) in West Glocester, RI, in which 60 fire experiments were conducted in a multiple-room enclosure to furnish validation data for theoretical fire models.

Figure 5.19 shows a diagram of the basic facility with indications of instrumentation location. The facility was built on the floor of FMRC's fire test building, using part of the $67 \mathrm{~m}$ by $76 \mathrm{~m}$ test building where the ceiling height is $18.3 \mathrm{~m}$. The layout in figure 25 shows a burn room and two target rooms connected to a corridor. The corridor was $2.43 \mathrm{~m}$ wide $\mathrm{x} 18.89 \mathrm{~m}$ long $\mathrm{x} 2.43 \mathrm{~m}$ high. The burn room measured $3.63 \mathrm{~m}$ deep x $3.64 \mathrm{~m}$ wide $\times 2.45 \mathrm{~m}$ high; a sealable window opening, measuring $0.85 \mathrm{~m}$ square, was centered on the rear wall, $0.34 \mathrm{~m}$ down from the top, and a door, measuring $0.92 \mathrm{~m}$ by $2.05 \mathrm{~m}$ high, was centered on the front wall (opening to the corridor). For closed window experiments, the wood-framed calcium silicate board window cover was pressed against a bead of caulking around the steel window frame and held by drop bars positioned into slots on the outside wall.

Room 3, located opposite the burn room, measured $3.65 \mathrm{~m}$ deep x $3.64 \mathrm{~m}$ wide $\times 2.45 \mathrm{~m}$ high; a door, measuring $0.88 \mathrm{~m}$ by $2.02 \mathrm{~m}$ high, was centered on the front wall (opening to the corridor). Room 4, located at the opposite end of the corridor, measured $3.65 \mathrm{~m}$ deep x $3.65 \mathrm{~m}$ wide $\times 2.43 \mathrm{~m}$ high and had a $0.88 \mathrm{~m}$ by $2.02 \mathrm{~m}$ high door centered on the front wall (opening to the corridor); an observation alcove, measuring $1.28 \mathrm{~m}$ by $0.86 \mathrm{~m}$ by $1.99 \mathrm{~m}$ high, was located in the front corner of room 4. Each room was equipped with a $102 \mathrm{~mm}$ inside diameter vent tube with a $61 \mathrm{~mm}$ inside diameter orifice meter and thermocouple, with option of exhaust fan (tube centered $0.27 \mathrm{~m}$ from the floor and $0.17 \mathrm{~m}$ from the closest parallel wall). An inlet vent $\left(0.29 \mathrm{~m}^{2}\right)$ used with exhaust fans was centered $0.43 \mathrm{~m}$ above the floor at the end of the corridor between the burn room and room 3 . When not in use, the inlet vent was sealed with a gypsum board cover taped in place.

The target room doors were commercial fire doors (wood-faced composite doors with calcium silicate cores, $14 \mathrm{~h}$ rated) mounted on 16 gage steel frames. The burn room door was fabricated from $12.7 \mathrm{~mm}$ calcium silicate, mounted in a steel frame lined with calcium silicate. Details of the 
doors and the spacings (cracks) are given in the original reference [70].

Gypsum wallboard, $12.7 \mathrm{~mm}$ thick, on wood studs was used throughout the experimental facility. In addition, the walls and ceiling of the burn room were overlaid with calcium silicate, also $12.7 \mathrm{~mm}$ thick, to harden against repeated fire exposure. The existing concrete floor of the test building was used.

Two types of fire sources were used: 1) steady propylene fires at $56 \mathrm{~kW}$ on a $0.30 \mathrm{~m}$ diameter sand burner and $522 \mathrm{~kW}$ on a $0.91 \mathrm{~m}$ diameter burner and 2) propylene fires on the $0.91 \mathrm{~m}$ diameter burner programmed under computer control to grow with the square of time, exceeding $1 \mathrm{MW}$ in $1,2,4$, or $8 \mathrm{~min}$.

The $0.91 \mathrm{~m}$ diameter, 0.58 in high propylene burner was used for most of the tests. Its design consisted of a 12 gage steel container with a gas distributor near the bottom, filled with gravel to a 67 percent height, where there was wire mesh screen, and coarse sand to the full height of the burner. The $0.30 \mathrm{~m}$ diameter burner was a scaled-down version of similar design.

\section{$5.8 \quad$ NIST Seven-story Hotel Tests}

By far the most complex test, this data set is part of a series of full-scale experiments conducted to evaluate zoned smoke control systems, with and without stairwell pressurization [71]. It was conducted in a seven story hotel with multiple rooms on each floor and a stairwell connecting all floors. This data set was chosen because it would challenge the scope of most current fire models. Measured temperatures and pressure differences between the rooms and floors of the building are extensive and consistent. Peak fire size was $3 \mathrm{MW}$ with a total building volume of $140000 \mathrm{~m}^{3}$.

Smoke movement and the performance of smoke control systems were studied in a seven story hotel building with smoke generated from wood fires and theatrical smoke. A total of 12 single experiments were conducted under a variety of conditions: two different fire sizes; sprinklered vs non-sprinklered wood fires; zoned smoke control on or off; stairwell pressurization on or off; with and without ventilation to the outside; and open and closed doors.

The Plaza Hotel building was a masonry structure consisting of two wings, one three stories and the other seven stories tall. The two wings were built at different times. The wings were connected to each other at only one location on each floor. The connections between the wings at each floor were sealed off, and the fires were set on the second floor of the seven-story wing, using the shorter wing as an instrumentation area. Areas of the second floor were fire hardened to minimize structural damage to the building.

The smoke control systems were designed using the methods presented in the smoke control manual of the American Society of Heating, Refrigerating, and Air-Conditioning Engineers [72], and the design analysis is discussed in detail by Klote [73]. The minimum design pressure difference was $25 \mathrm{~Pa}$, meaning that the system should be able to maintain at least this value without a fire. The Plaza Hotel building had no central forced air heating, ventilating, and air-conditioning (HVAC) system, so a dedicated system of fans and ducts was installed for zoned smoke control and stairwell pressurization. The smoke control system consisted of the three $0.944 \mathrm{~m}^{3} / \mathrm{s}$ centrifugal fans shown in 5.20, plus another centrifugal fan (not shown) located outside and supplying 4.25

$\mathrm{m}^{3} / \mathrm{s}$ of pressurization air to the stairwell at the first floor. The smoke control system is illustrated in figure 5.20. All the test fires were located in the second floor smoke zone. This smoke was exhausted at about six air changes per hour. The first and second floors were pressurized at about six 

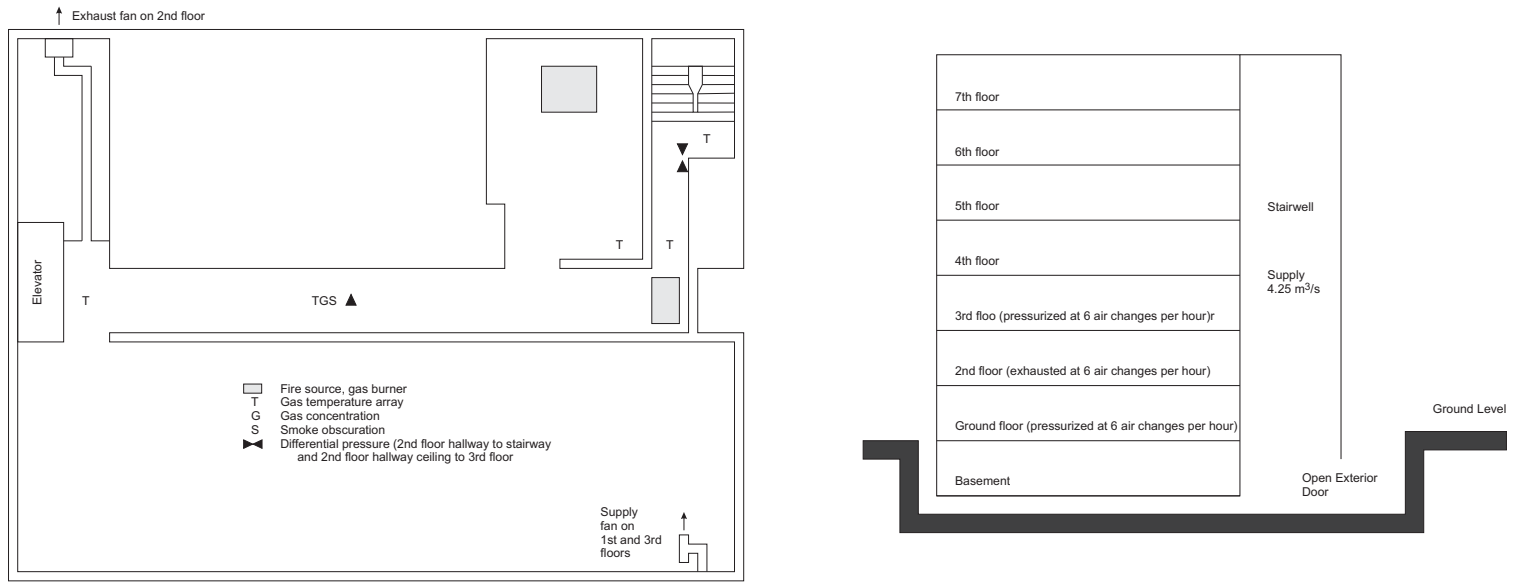

Figure 5.20: Overview of the NIST Seven-story hotel test series including smoke control.

air changes per hour. When the stairwell pressurization system was activated, the exterior stairwell door was open. This approach is intended to minimize fluctuations due to opening and closing doors.

\subsection{NIST / Navy High Bay Hanger Tests}

Thirty-three full-scale experiments were conducted using JP-5 and JP-8 fuels in two Navy high bay aircraft hangars located at the Naval Air Stations in Barber's Point, Hawaii and Keflavik, Iceland [74, 75]. Both facilities were very large single compartments with and without draft curtains. Compartment size were $97.8 \mathrm{~m}$ by $73.8 \mathrm{~m}$ by $14.9 \mathrm{~m}$ high in Hawaii and $73.8 \mathrm{~m}$ by $45.7 \mathrm{~m}$ by $22.3 \mathrm{~m}$ high in Iceland. Both hangars were constructed with walls of concrete masonry, steel, and glass. with large doors at two ends of each facility (kept closed for the tests used in this report). Ceilings of both structures were steel, with a suspended tile ceiling below the steel shell in Iceland. In addition to numerous detection and suppression devices, temperature measurements in and around the fire plume allowed characterization of the fire plume. Fire height was also reported for the tests. The full-scale test fires produced heat release rates ranging from $100 \mathrm{~kW}$ to $33 \mathrm{MW}$ using JP-5 and JP-8 jet fuel as the fire source contained in pans of various sizes. 


\section{Chapter 6}

\section{Hot Gas Layer Temperature and Depth}

CFAST simulated all of the chosen experiments. Details of the comparisons with experimental data, are provided in Appendix A. The results are organized by quantity as follows:

- hot gas layer (HGL) temperature and height

- ceiling jet temperature

- plume temperature

- flame height

- oxygen and carbon dioxide concentration

- smoke concentration

- compartment pressure

- radiation heat flux, total heat flux, and target temperature

- wall heat flux and surface temperature

Comparisons of the model predictions with experimental measurements are presented as relative differences. The relative differences are calculated as follows:

$$
\varepsilon=\frac{\Delta M-\Delta E}{\Delta E}=\frac{\left(M_{P}-M_{0}\right)-\left(E_{P}-E_{0}\right)}{\left(E_{P}-E_{0}\right)}
$$

where $\Delta M$ is the difference between the peak value $\left(M_{P}\right)$ of the evaluated parameter and its original value $\left(M_{0}\right)$, and $\Delta E$ is the difference between the experimental observation $\left(E_{P}\right)$ and its original value $\left(E_{0}\right)$.

The measure of model accuracy used throughout this study is related to experimental uncertainty. Reference [60] discusses this issue in detail. In brief, the accuracy of a measurement, for example, a gas temperature, is related to the measurement device, a thermocouple. In addition, the accuracy of the model prediction of the gas temperature is related to the simplified physical description of the fire and the accuracy of the input parameters, especially the specified heat release rate. Ideally, the purpose of a validation study is to determine the accuracy of the model in 
the absence of any errors related to the measurement of both its inputs and outputs. Because it is impossible to eliminate experimental uncertainty, at the very least a combination of the uncertainty in the measurement of model inputs and output can be used as a yardstick. Dotted lines in figure 6.1 show this combined uncertainty estimate for HGL temperature and layer depth. Corresponding estimates are included for other quantities in later sections. If the numerical prediction falls within the range of uncertainty attributable to both the measurement of the input parameters and the output quantities, it is not possible to further quantify its accuracy. At this stage, it is said that the prediction is within experimental uncertainty.

Note that the calculation of relative difference is based on the temperature rise above ambient, and the layer depth, that is, the distance from the ceiling to where the hot gas layer descends. Where the model over-predicts the HGL temperature or the depth of the HGL, the relative difference is a positive number. This convention is used throughout this report where the model over-predicts the severity of the fire, the relative difference is positive; where it under-predicts, the difference is negative.

Arguably the most frequent question asked about a fire is, How hot did it become? Temperature in the upper layer of a compartment is an obvious indicator to answer this question. Peak temperature, time to peak temperature, or time to reach a chosen temperature tenability limit are typical values of interest. Quality of the prediction (or measurement) of layer interface position is more difficult to quantify. Although observed in a range of experiments, the two-layer assumption is in many ways just a convenience for modeling. In experimental measurements, temperature is typically measured with an array of thermocouples from floor to ceiling. This floor to ceiling temperature profile can then used to estimate a hot gas layer height and the temperature of the upper and lower gas layers [76] [77] consistent with the two-zone assumption. Appendix A provides details of the calculation.

From a standpoint of hazard, time of descent to a chosen level may be a reasonable criterion (assuming some in the room will then either be forced to crawl beneath the interface to breathe the clean atmosphere near the floor or be forced to breath the upper layer gases). Minimum values may also be used to indicate general agreement. For the single-room tests with furniture or wallburning, these are appropriate indicators to judge the comparisons between model and experiment. For the more-closely steady-state three- and four-room tests with corridor or the multiple-story building tests, a steady-state average may better characterizes the nature of the experiment.

A good prediction of the HGL height is largely a consequence of a good prediction of its temperature because smoke and heat are largely transported together and most numerical models describe the transport of both with the same type of algorithm. Typically, CFAST slightly overpredicts the HGL temperature, most often within experimental uncertainty. Hot gas layer height is typically within experimental uncertainty for well-ventilated tests and near floor level for underventilated tests where compartments are closed to the outside. For HGL height, only values from open-door tests are included. For closed-door tests, visual observations typically show that the HGL fills the entire compartment volume from floor to ceiling, inconsistent with the calculated results for the experimental data. Thus, the calculated experimental values of HGL height for closed-door tests are not seen as appropriate for comparison to model results. 

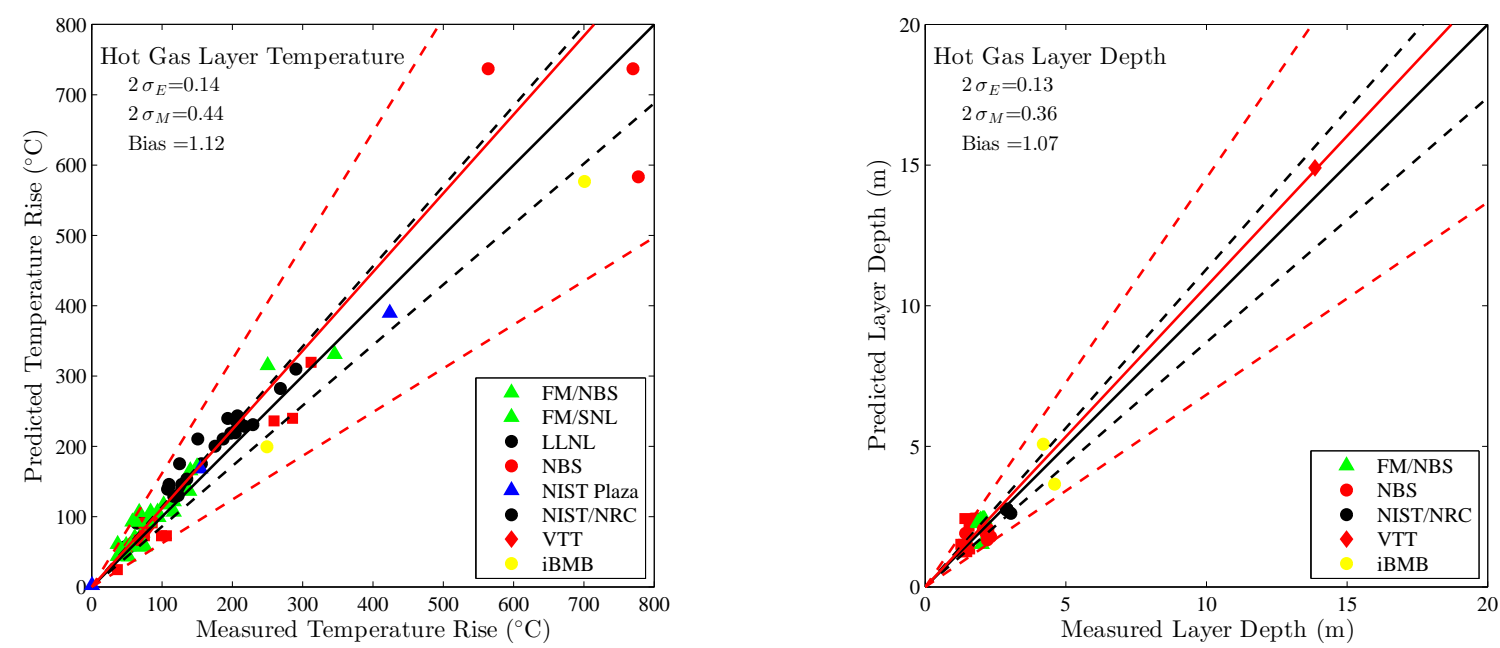

Figure 6.1: Comparison of Measured and Predicted HGL Temperature and Height.

\subsection{Model / Experiment Comparisons}

Figure 6.1 shows a comparison of predicted and measured values for HGL temperature and depth. Appendix A provides individual graphs of model and experimental values and tables of peak values and relative differences.

Following is a summary of the accuracy assessment for the HGL predictions of the test series:

\section{NBS Single Room Tests with Furniture}

For two of the four tests (Tests F1 and F6, tests with a single furniture item in the compartment), the HGL temperature and depth for these tests were calculated separately for two different thermocouple arrays, one located in the front of the compartment and one in the rear. For the other two tests (tests W1 and W2, tests with furniture and a wood wall surface), a single thermocouple array was available.

For the single-room tests, predicted temperatures and layer interface position show obvious similarities to the measured values (see, for example, figure 6.2). Peak values occurred at similar times with comparable rise and fall for most comparisons. Interface height for the single-room with wall-burning is a notable exception. Unlike the model prediction, the experimental measurement did not show the rise and fall in concert with the temperature measurement. Peak values were typically higher for upper layer temperature and lower for lower layer temperature and layer interface position.

For the furniture tests, the agreement ranges from a relative difference of $-43 \%$ to $31 \%$. The agreement is quite different for the two arrays in each test. Table 6.1 shows the relative differences for tests F1 and F6. For both the layer temperature and layer depth, the difference between the model and experiment changes depending on the measurement location. In the same test (F1) the values are well within experimental uncertainty at one location and outside for the other. For test F6, the differences are greater. For these tests with a post-flashover fire in a small compartment, the 

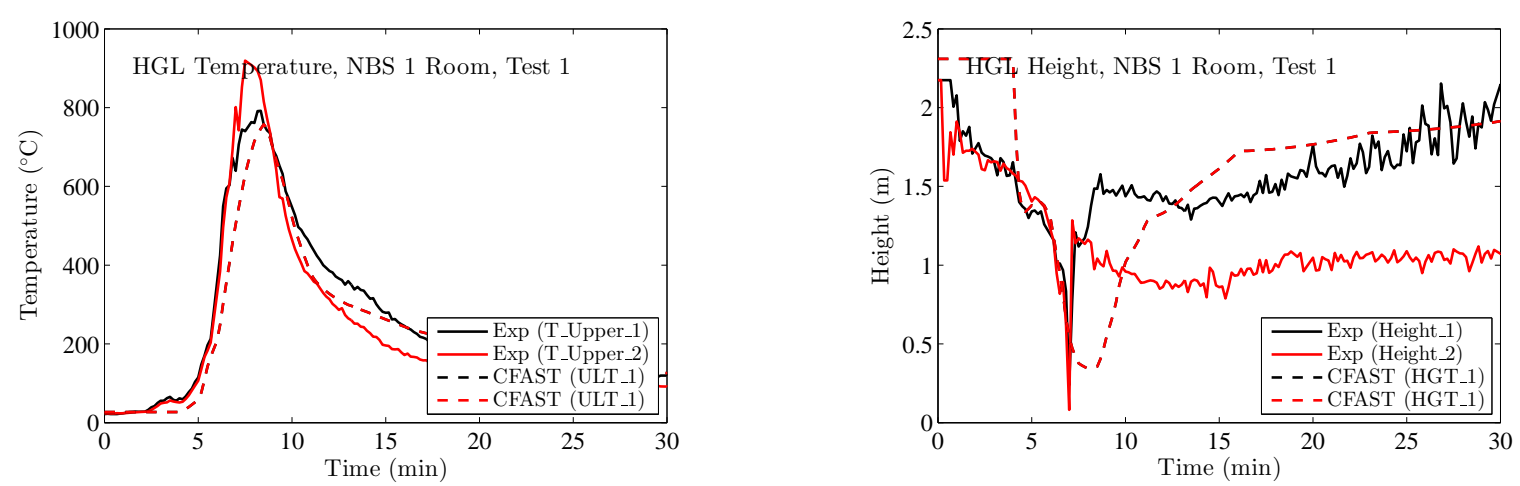

Figure 6.2: Measured and Predicted HGL Temperature and Height for a Single Compartment Test.

Table 6.1: Relative Difference for HGL Temperature and Depth for Two Measurement Locations in Two Single-Room Tests

\begin{tabular}{|l|c|c|c|}
\hline Test & Location & $\begin{array}{c}\text { Temperature } \\
\%\end{array}$ & $\begin{array}{c}\text { Layer Depth } \\
\%\end{array}$ \\
\hline \hline \multirow{2}{*}{ F1 } & A & -4 & -1 \\
& B & -18 & -8 \\
\hline \multirow{2}{*}{ F6 } & A & 31 & -8 \\
& B & -16 & -43 \\
\hline
\end{tabular}

assumption of a uniform upper layer may be questionable. Still, curve shape for the experimental data and model predictions is quite similar.

\section{VTT Large Hall Test Series}

The HGL temperature and depth were calculated from the averaged gas temperatures from three vertical thermocouple arrays. Ten thermocouples in each vertical array, spaced $2 \mathrm{~m}$ apart in the lower two-thirds of the hall, and $1 \mathrm{~m}$ apart near the ceiling were used to determine layer temperatures and depth.

CFAST predicts the HGL temperature and height near experimental uncertainty for all three tests, with relative differences ranging from $6 \%$ to $10 \%$, depending on the test. Relative differences are shown in figure 6.1 .

\section{NIST/NRC Test Series}

The NIST/NRC series consisted of 15 liquid spray fire tests with different heat release rates, pan locations, and ventilation conditions. Gas temperatures were measured using seven floor-to-ceiling thermocouple arrays (or "trees") distributed throughout the compartment. The average hot gas layer temperature and height were calculated using thermocouple Trees 1, 2, 3, 5, 6 and 7. Tree 4 was not used because one of its thermocouples malfunctioned during most of the experiments. 
A few observations about the simulations:

- In the closed-door tests, the HGL layer descended all the way to the floor. However, the reduction method used on the measured temperatures (see reference [77]) does not account for the formation of a single layer and, therefore, does not indicate that the layer dropped all the way to the floor, rather just to the position of the lowest temperature measurement point. This is not a flaw in the measurements, but rather that the data reduction method only applies to tests where two distinct layers are present, i.e., in the open door tests.

- The HGL reduction method produces spurious results in the first few minutes of each test because no clear layer has yet formed.

CFAST predicts the HGL temperature to within experimental uncertainty for all of the closeddoor tests. Relative differences for the open-door tests are somewhat higher, ranging from $9 \%$ for Test 15 to $24 \%$ for Test 18 . CFAST predicts HGL height to within experimental uncertainty for the open-door tests.

\section{FM/SNL Test Series}

Tests 4, 5, and 21 from the FM/SNL test series are selected for comparison. The hot gas layer temperature and height are calculated using the standard method. The thermocouple arrays that are referred to as Sectors 1, 2 and 3 are averaged (with an equal weighting for each) for Tests 4 and 5. For Test 21, only Sectors 1 and 3 are used, as Sector 2 falls within the smoke plume.

Note the following:

- The experimental HGL heights are somewhat noisy because of the effect of ventilation ducts in the upper layer. The corresponding predicted HGL heights are consistently lower than experimental measurements, typically approaching floor level by the end of the test. This is likely a combination of the calculation technique for the experimental measurements and rules for flow from mechanical vents in the CFAST model.

- The ventilation was turned off after 9 minutes in Test 5, the effect of which was a slight increase in the measured HGL temperature.

CFAST predicts the HGL temperature well within experimental uncertainty for Tests 4 and 5, but overpredicts for Test 21. This is likely because of the configuration of the fire in the test, with the fire inside a cabinet in the fire compartment. This complex geometry leads to an interaction between the fire and the confining cabinet that a zone model cannot simulate.

\section{iBMB Compartment Tests}

CFAST predicts the HGL temperature and height somewhat outside experimental uncertainty with some discrepancy in the shapes of the curves. It is not clear whether this is related to the measurement or the model. For the cable fire test (Test 4), this is likely because of the complicated geometry within the compartment that includes a partial height wall that affects both plume entrainment and radiative heat transfer from the fire to surroundings. 


\section{NBS Multi-Room Test Series}

This series of experiments consists of two relatively small rooms connected by a long corridor. The fire is located in one of the rooms. Eight vertical arrays of thermocouples are positioned throughout the test space: one in the burn room, one near the door of the burn room, three in the corridor, one in the exit to the outside at the far end of the corridor, one near the door of the other or "target" room, and one inside the target room. Four of the eight arrays have been selected for comparison with model prediction: the array in the burn room (BR), the array in the middle of the corridor (5.5 $\mathrm{m}$ from the BR), the array at the far end of the corridor (11.6 $\mathrm{m}$ from the BR), and the array in the target room (TR). In Tests $100 \mathrm{~A}$ and $100 \mathrm{O}$, the target room is closed, in which case the array in the exit (EXI) doorway is used. The test director reduced the layer information individually for the eight thermocouple arrays using an alternative method. These results are included in the original data sets. However, for the current validation study, the selected TC trees were reduced using the method common to all the experiments considered.

CFAST predicts the HGL temperature and depth to within experimental uncertainty for many of the measurement locations in the three tests considered, averaging within $14 \%$ for the HGL temperature and $21 \%$ for the HGL depth. The discrepancies in various locations appear to be attributable to experimental, rather than model, error. In particular, the calculation of HGL temperature and height are quite sensitive to the measured temperature profile, which in these tests was determined with bare-bead thermocouples that are subject to quite high uncertainties. Wide spacing of the thermocouples also leads to higher uncertainty in HGL height.

Calculations of HGL temperature and depth in rooms remote from the fire tend to higher relative differences than those closer to the fire. This is likely a combination of the simplified single representative layer temperature inherent in zone models (temperature in the long corridor of this test series varied from one end of the compartment to the other), calculation of flow though doorways based on a correlation based on the pressure difference between the connected compartments, and unknown leakage from each the compartments that would effect these pressure differences.

\section{FM Four Room Including Corridor Test Series}

This series of experiments has a burn room connected to a long corridor with two smaller compartments at the far end of the corridor. Six vertical arrays of thermocouples (one in most compartments and three in the corridor) were used to estimate layer temperature and depth. In general, CFAST over predicts layer temperature and depth closer to the fire with a general trend towards under prediction at locations more remote from the fire; at times within experimental uncertainty but with significant over prediction at other locations. Relative differences averaged within $14 \%$ for HGL temperature and within $21 \%$ for HGL depth.

\section{NIST Seven-story Hotel Tests}

These tests were conducted in a seven story hotel with multiple rooms on each floor and a stairwell connecting all floors. Layer temperatures are available for comparison directly from the data. Relative differences comparing model and experiment are well within experimental uncertainty except on the seventh floor. While the relative difference for this location was quite large (292\%, this represents a $2{ }^{\circ} \mathrm{C}$ over prediction by the model. 


\subsection{Summary}

Following is a summary of the accuracy assessment for the HGL predictions. The two-zone assumption inherent in CFAST, modeled as a series of ordinary differential equations that describe mass and energy conservation of flows in a multiple-compartment structure typically provide appropriate prediction of gas layer temperature and layer height for the applications studied.

- The CFAST predictions of the HGL temperature and height are, with a few exceptions, within or close to experimental uncertainty. On average, predicted HGL temperature is within $14 \%$ and HGL depth is within $21 \%$ of experimental measurements. The CFAST predictions are typical of those found in other studies where the HGL temperature is typically somewhat over-predicted and HGL height somewhat lower (HGL depth somewhat thicker) than experimental measurements. These differences are likely attributable to simplifications in the model dealing with mixing between the layers, entrainment in the fire plume, and flow through vents.

- Calculation of HGL temperature and height has higher uncertainty in rooms remote from the fire compared to those in the fire compartment. Most likely, this is due to a combination of the simplified vent flow predictions (based on idealized Bernoulli flow) and the assumption of constant compartment surface thermal properties that are assumed independent of temperature. 


\section{Chapter 7}

\section{Flame Height, Plume Temperature and Ceiling Jets}

\subsection{Flame Height}

Flame height is recorded by visual observations, photographs, or video footage. Videos from the NIST/NRC test series and photographs from the VTT Large Hall Test Series are available. It is difficult to precisely measure the flame height, but the photos and videos allow one to make estimates accurate to within a pan diameter.

\section{VTT Large Hall Test Series}

The height of the visible flame in the photographs has been estimated to be between 2.4 and 3 pan diameters $(3.8 \mathrm{~m}$ to $4.8 \mathrm{~m})$. From the CFAST calculations, the estimated flame height is $4.3 \mathrm{~m}$.

\section{NIST/NRC Test Series}

CFAST estimates the peak flame height to be $2.8 \mathrm{~m}$, consistent with the roughly $3 \mathrm{~m}$ flame height observed through the doorway during the test. The test series was not designed to record accurate measurements of flame height.

\section{NIST/Navy High Bay Hangar Test Series}

For the 9 Iceland tests, CFAST predicts flame height within $25 \%$ of the experimentally reported values, with the largest relative differences for the smaller heat release rate fires. Uncertainty in the flame height measurements for the experiments was reported to be $\pm 0.5 \mathrm{~m}$, approaching $30 \%$ of the experimental values for the lower heat release rate fires.

\subsection{Plume Temperature}

As with the ceiling jet, CFAST includes a specific plume temperature model based on the McCaffrey plume [78, 79] to account for presence of higher gas temperatures near a target located 


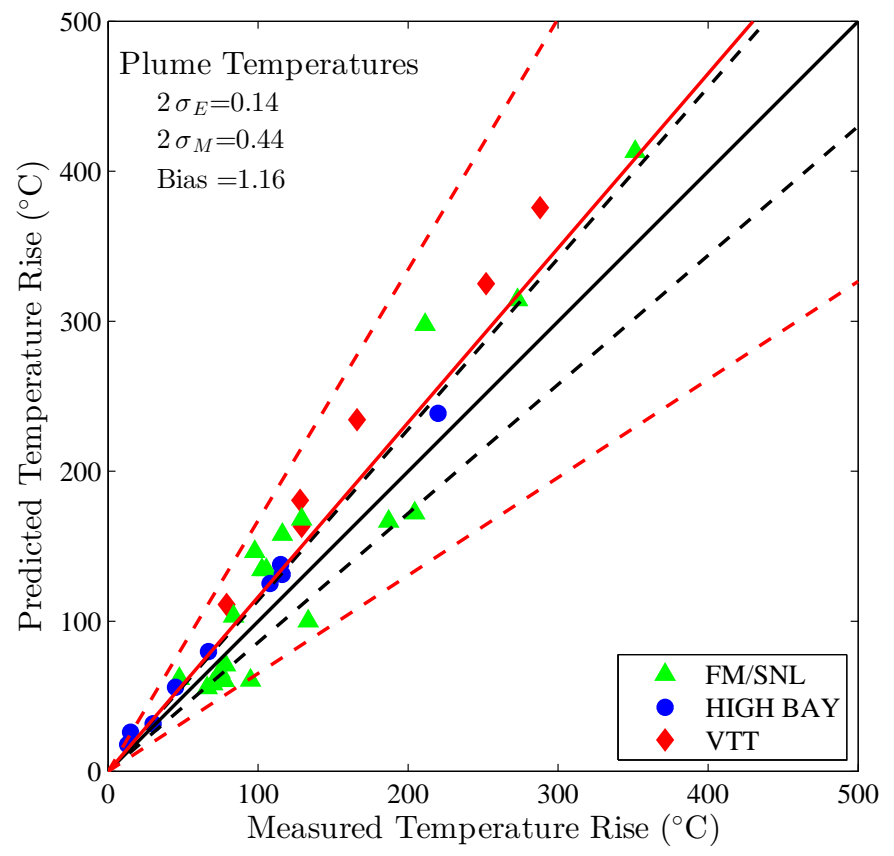

Figure 7.1: Comparison of Measured and Predicted Plume Centerline Temperature.

at the centerline of the fire plume. the correlation has been subjected to extensive validation efforts by McCaffrey [78] and others [23] and shown to provide predictions within about $30 \%$ of a wide range of experimental results [23]. In the model, this increased temperature has the effect of increasing the convective heat transfer to the target. Only two of the six test series (VTT and $\mathrm{FM} / \mathrm{SNL}$ ) included measurements of plume centerline temperature.

Figure 7.1 shows a comparison of predicted and measured values for plume temperature. Appendix A provides individual graphs of model and experimental values. All of the comparisons are to the surrounding gas temperature predicted by CFAST. Comparisons to the target surface temperature or target center temperature would be expected to have a smaller relative difference since all the predictions of surrounding gas temperature are higher than experimental measurements. Following is a summary of the accuracy assessment for the ceiling jet predictions in the two test series.

\section{VTT Test Series}

With one exception, CFAST predicts plume temperature within experimental uncertainty for the experiments in this test series. The predictions average within $10 \%$ of the experimental measurements. 


\section{FM/SNL Test Series}

For the tests in this test series, predictions are near experimental uncertainty (averaging within $13 \%$ of experimental measurements). This does not include results from test 21 since this test was a fire inside an electrical equipment cabinet that did not form a well-defined plume within the larger compartment.

\section{NIST / Navy High Bay Hangar Test Series}

Predictions for the high bay tests average within $30 \%$. With a wide range of fire sizes (from $1.4 \mathrm{MW}$ to $33 \mathrm{MW}$ ), this test series provides a broad evaluation of the underlying correlations used in the model. The two tests with the highest relative difference are the lowest heat release rate tests. With all plume temperature measurements made at a height of more than $22 \mathrm{~m}$, a higher uncertainty for the lowest heat release rate tests is understandable.

\subsection{Ceiling Jets}

CFAST includes an algorithm to account for the presence of the higher gas temperatures near the ceiling surfaces in compartments involved in a fire. In the model, this increased temperature has the effect of increasing the convective heat transfer to ceiling surfaces. The temperature and velocity of the ceiling jet are available from the model by placing a heat detector at the specified location. The ceiling jet algorithm is based on the model by Cooper [80], with details described in the CFAST Technical Reference Guide [2]. The algorithm predicts gas temperature and velocity under a flat, unconstrained ceiling above a fire source. Only two of the six test series (NIST/NRC and FM/SNL) involved relatively large flat ceilings.

Figure 7.2 shows a comparison of predicted and measured values for ceiling jet temperature. Appendix A provides individual graphs of model and experimental values. Following is a summary of the accuracy assessment for the ceiling jet predictions in the two test series.

\section{NIST/NRC Test Series}

The thermocouple nearest the ceiling in Tree 7, located towards the back of the compartment, has been chosen as a surrogate for the ceiling jet temperature. This location was well removed from the fire plume so that plume effects would not be evident, but closer to the wall surfaces so that the assumption of an unconfined ceiling inherent in the typical ceiling jet correlations that wall effects may impact the comparison. Still, CFAST predicts ceiling jet temperature well within experimental uncertainty for all but one of the tests in the series, with an average relative difference of $5 \%$. For these tests, the fire source was sufficiently large (relative to the compartment size) such that a well-defined ceiling jet was evident in temperature measurements near ceiling level.

\section{FM/SNL Test Series}

With fire sizes comparable to the smaller fire sizes used in the tests in NIST/NRC test series and compartment volumes significantly larger, measured temperature rise near the ceiling in the FM/SNL tests was below $100{ }^{\circ} \mathrm{C}$ in all three tests. Relative differences averaged within $19 \%$ of 


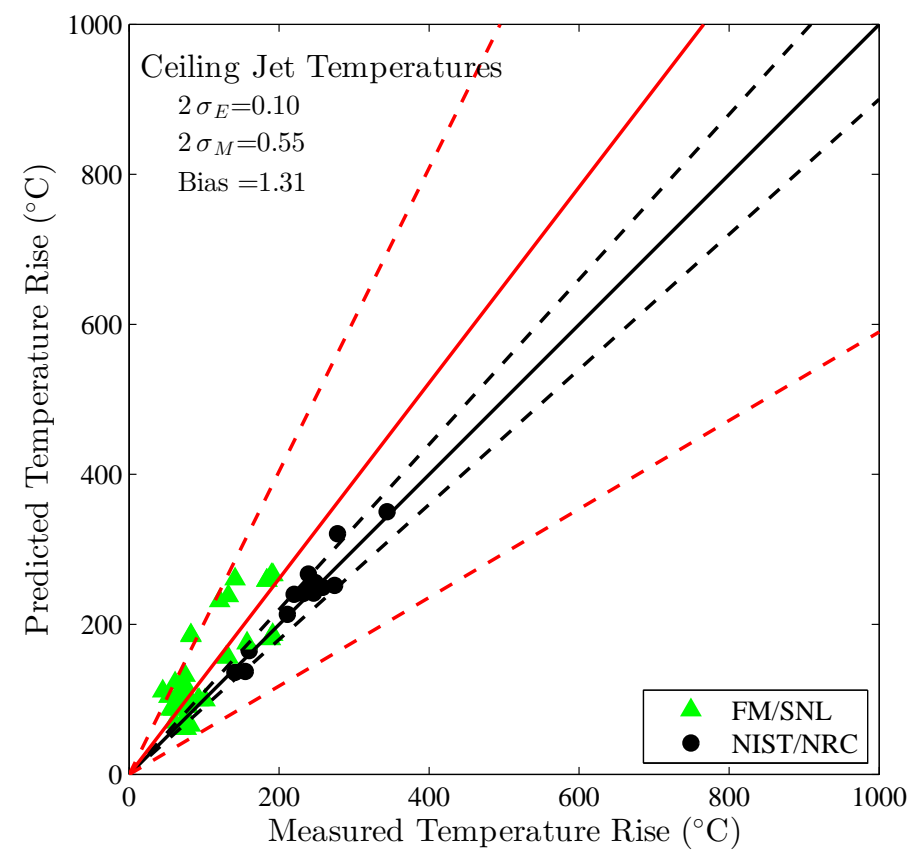

Figure 7.2: Comparison of Measured and Predicted Ceiling Jet Temperature. 
experimental measurements. Hot gas layer temperatures for these tests were below $70{ }^{\circ} \mathrm{C}$. CFAST consistently predicts higher ceiling jet temperatures in the FM/SNL tests compared to experimental measurements. With a larger compartment relative to the fire size, the ceiling jet for the FM/SNL tests is not nearly as well-developed as those in the NIST/NRC tests. The difference between the experimental ceiling jet temperature and HGL temperature for the FM/SNL tests is less than half that observed in the NIST/NRC tests. While the over-prediction of ceiling jet temperature could be considered conservative for some applications, for scenarios involving sprinkler or heat detector activation, the increased temperature in the ceiling jet would lead to shorter estimates of activation times for the simulated sprinkler or heat detector.

\subsection{Summary}

Based on the model physics and comparisons of model predictions with experimental measurements, CFAST provides appropriate calculations of flame height for the following reasons:

- The correlation used in CFAST to predict plume temperature is well-suited for this application and has been subjected to extensive comparisons with experimental results.

- CFAST provides predictions consistent with visual observation for available data in this validation exercise.

Based on the model physics and comparisons of model predictions with experimental measurements, the use of CFAST to predict plume temperature requires caution for the following reasons:

- The correlation used in CFAST to predict plume temperature is well-suited for this application and has been subjected to extensive validation efforts.

- CFAST tends to over-predict plume temperatures comparing experimental measurements to gas temperature predictions by the model. Comparisons to target surface or center temperature (which may be appropriate for unshielded thermocouple measurements in experiments) show closer agreement.

- $90 \%$ of the predictions by CFAST are within $43 \%$ of experimental measurements, higher than the estimated experimental uncertainty in this validation exercise, but this is largely driven by a few individual tests. User's should take this higher uncertainty into account when using predictions from the model.

Based on the model physics and comparisons of model predictions with experimental measurements, CFAST provides appropriate calculations of ceiling jet temperature for the following reasons:

- For tests with a well-defined ceiling jet layer beneath flat ceilings, CFAST predicts ceiling jet temperatures well-within experimental uncertainty.

- For tests with a less well-defined ceiling jet layer, CFAST over-predicts the ceiling jet temperature. For the tests studied, over-predictions were noted when the HGL temperature was below $70{ }^{\circ} \mathrm{C}$. 


\section{Chapter 8}

\section{Gas Species and Smoke}

CFAST simulates a fire as a mass of fuel that burns at a prescribed pyrolysis rate and releases both energy and combustion products. CFAST calculates species production based on user-defined production yields, and both the pyrolysis rate and the resulting energy and species generation may be limited by the oxygen available for combustion. When sufficient oxygen is available for combustion, the heat release rate for a constrained fire is the same as for an unconstrained fire. Mass and species concentrations, assumed to be homogeneous throughout each layer, are tracked by the model as gases flow through openings in a structure to other compartments in the structure or to the outdoors.

The fire chemistry scheme in CFAST is essentially a species balance from user-prescribed species yields and the oxygen available for combustion. Once generated, it is a matter of bookkeeping to track the mass of species throughout the various control volumes in a simulated building. It does, however, provide a check of the flow algorithms within the model. Since the major species $\left(\mathrm{CO}\right.$ and $\left.\mathrm{CO}_{2}\right)$ are generated only by the fire, the relative accuracy of the predicted values throughout multiple rooms of a structure should be comparable.

\subsection{Oxygen and $\mathrm{CO}_{2}$}

Gas sampling data are available from a number of the experimental tests. Figure 8.1 shows a comparison of predicted and measured values for oxygen and carbon dioxide concentrations, along with a summary of the relative difference for the tests.

CFAST predicts the upper-layer concentrations of oxygen and carbon dioxide within an average of $22 \%$ of experimental values. Details for individual test series are discussed below.

\section{NBS Single Room Tests with Furniture}

For the single-room tests with furniture, the predicted concentrations are lower than those measured experimentally (with an average relative difference of $22 \%$ for oxygen. This is probably due to the treatment of oxygen limited burning. In CFAST, the burning rate simply decreases as the oxygen level decreases. A user prescribed lower limit determines the point below which burning will not take place. This parameter could be finessed to provide better agreement with the experiment. For the present comparisons, it was always left at the default value. Since this parameter impacts the 

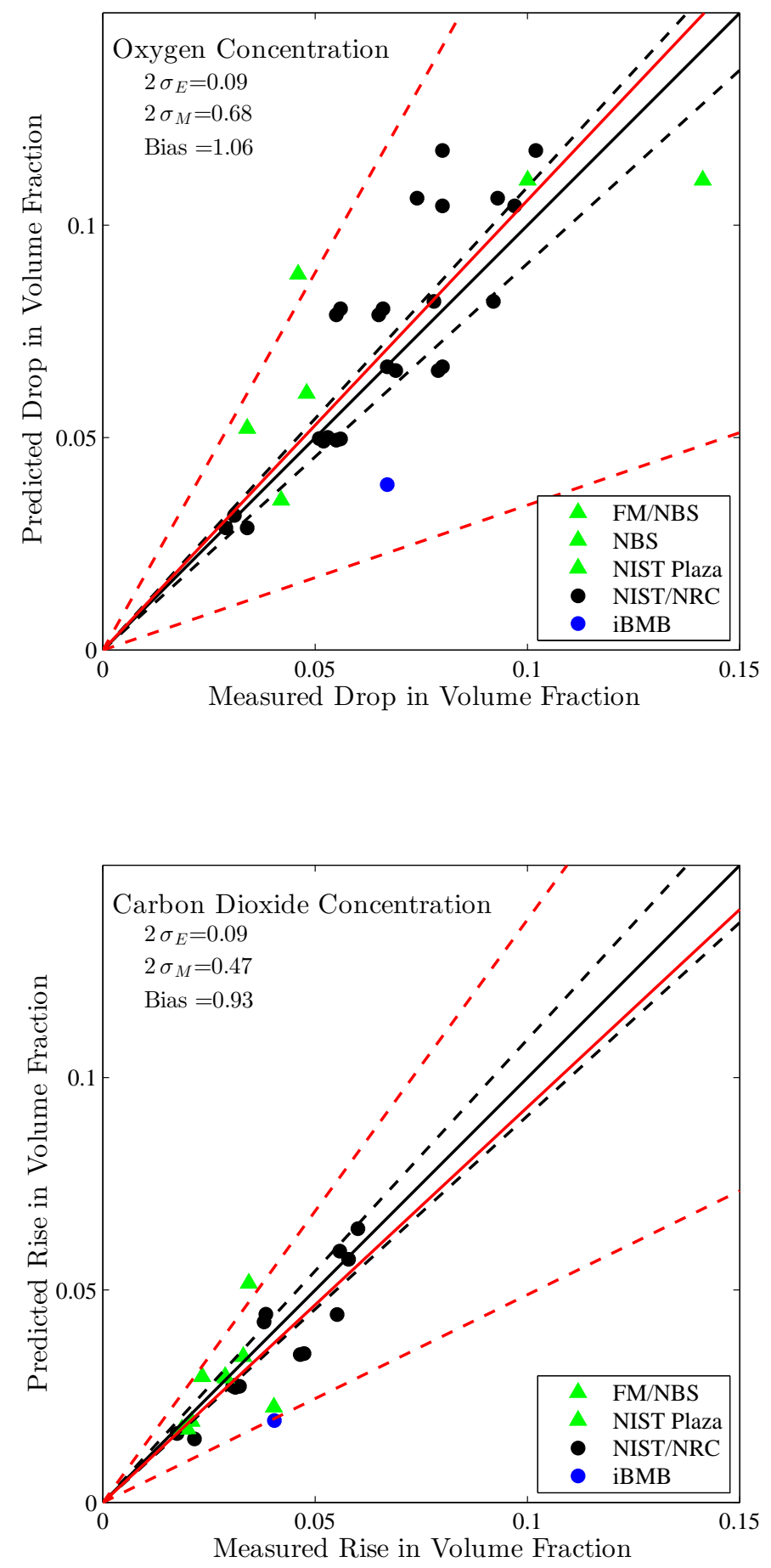

Figure 8.1: Comparison of Measured and Predicted Oxygen Concentration and Carbon Dioxide Concentration. 
overall combustion chemistry in CFAST and the generation of all species, it would impact both oxygen and carbon dioxide.

In addition, species concentrations measured for large fires in small spaces can show considerable spatial variation within the upper layer of the fire compartment [81]. For such under-ventliated fires, the representation of the species concentration in a hot gas layer by a single representative average value may not be valid. For example, two measurement locations in test F6 show and underprediction in one measurement location and an overprediction in the other.

\section{NIST/NRC Tests}

Relative differences for both oxygen and carbon dioxide are typically higher for the closed door tests than for the open door tests (see figure 8.1). Tests 4, 10, and 16 were closed-door tests with the mechanical ventilation system on. A higher relative differences for these tests are likely because of a non-uniform gas layer in the experiments with higher oxygen concentration near the mechanical ventilation inlet and lower concentrations remote from the inlet. In CFAST, the flow from the mechanical ventilation system is assumed to completely mix with the gases in the appropriate gas layer of a compartment. For other tests, the reasons for a higher relative difference are not clear. Replicate tests are seen to typical compare quite well, with a difference of about $2 \%$ typical.

Table 8.1: Relative Difference for Oxygen and Carbon Dioxide in Open and Closed Door Tests in a Single Compartment

\begin{tabular}{|l|c|c|}
\hline Vent & $\begin{array}{c}\text { Oxygen } \\
\%\end{array}$ & $\begin{array}{c}\text { Carbon Dioxide } \\
\%\end{array}$ \\
\hline \hline Open Door & 7 & 13 \\
\hline Closed Door & 20 & 15 \\
\hline
\end{tabular}

\section{iBMB Cable Fire Test}

CFAST under predicts oxygen and carbon dioxide concentrations by $58 \%$ and $66 \%$, respectively.

\section{FM Four Room Including Corridor Test Series}

For these four compartment tests, the end of test values of the gas concentrations agree far better than the peak values. With the underlying assumption of all zone fire models of a uniform concentration of gas species throughout a control volume, it is assumed than the point measurement is the bulk concentration of the entire upper layer. In reality, some vertical distribution not unlike the temperature profile exists for the gas concentration as well.

Since this measurement point is near the lower edge of the upper layer for a significant time, it should underestimate the bulk concentration until the layer is large in volume and well mixed. Still, the relative differences for these tests are larger than the NIST/NRC tests, averaging $54 \%$ for oxygen and $22 \%$ for carbon dioxide. 


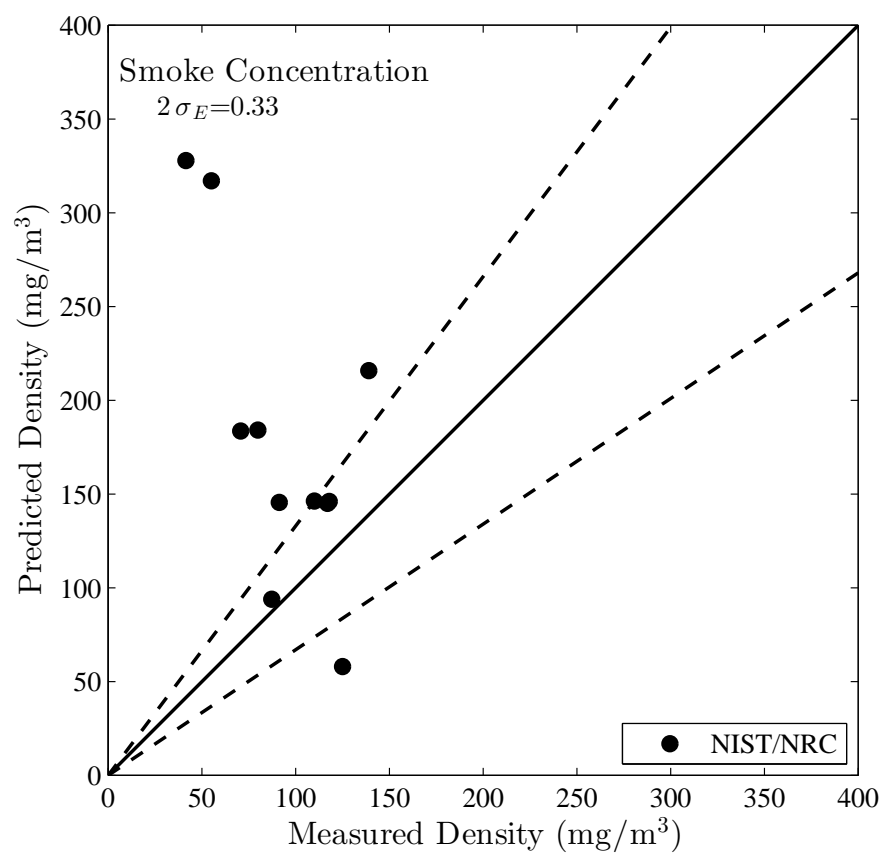

Figure 8.2: Comparison of Measured and Predicted Smoke Concentration.

\section{NIST Seven-story Hotel Tests}

For the multiple-story building test, predicted values for $\mathrm{CO}_{2}, \mathrm{CO}$, and $\mathrm{O}_{2}$ are lower than measured experimentally. Both the lower burning rate limit as well as leakage in the 100 year- old structure probably contributed to the differences between the experiments and model. In addition, values for species yields were simply literature values since no test data were available.

\subsection{NIST/NRC Test Series, Smoke}

CFAST treats smoke like all other combustion products, with an overall mass balance dependent on interrelated user-specified species yields for major combustion species. To model smoke movement, the user prescribes the smoke yield relative to the yield of carbon monoxide. A simple combustion chemistry scheme in the model then determines the smoke particulate concentration in the form of an optical density. Figure 8.2 shows a comparison of predicted and measured values for smoke concentration along with a summary of the relative difference for the tests.

Only the NIST/NRC test series has been used to assess predictions of smoke concentration. For these tests, the smoke yield was specified as one of the test parameters. There are two obvious trends in the results. First, the predicted concentrations are within or near experimental uncertainties in the open-door tests. Second, the predicted concentrations are roughly three to five times the measured concentrations in the closed-door tests. The experimental uncertainty for these mea- 
surements has been estimated to be $33 \%$. The closed-door tests cannot be explained from the experimental uncertainty.

The difference between model and experiment is far more pronounced in the closed-door tests. Given that the oxygen and carbon dioxide predictions are no worse (and indeed even better) in the closed-door tests, there is reason to believe either that the smoke is not transported with the other exhaust gases or the specified smoke yield, developed from free-burning experiments, is not appropriate for the closed-door tests. These qualitative differences between the open- and closeddoor tests are consistent with the FDS predictions (see reference [82]).

\subsection{Summary}

Based on the model physics and comparisons of model predictions with experimental measurements, CFAST calculations of oxygen and carbon dioxide concentration are seen as appropriate with the following comments:

- CFAST uses a simple user-specified combustion chemistry scheme based on a prescribed pyrolysis rate and species yields that is appropriate for the applications studied.

- CFAST predicts the major gas species to within $22 \%$ of experimental measurements.

- For large fires in small compartments or where mixing within a compartment may be important, local species concentration may vary considerably from a bulk average value. Thus higher uncertainty can be expected for predictions of species concentrations in these scenarios.

Use of CFAST calculations of smoke concentration require additional care for the following reasons:

- CFAST is capable of transporting smoke throughout a compartment, assuming that the production rate is known and its transport properties are comparable to gaseous exhaust products.

- CFAST typically over-predicts the smoke concentration in all of the NIST/NRC tests, with the exception of Test 17. Predicted concentrations for open-door tests are within experimental uncertainties, but those for closed-door tests are far higher. No firm conclusions can be drawn from this single data set. The measurements in the closed-door experiments are inconsistent with basic conservation of mass arguments, or there is a fundamental change in the combustion process as the fire becomes oxygen-starved. 


\section{Chapter 9}

\section{Pressure}

Comparisons between measurement and prediction of compartment pressure for the NIST/NRC test series and two of the NBS furniture tests are shown in of Appendix A. Figure 9.1 shows a comparison of predicted and measured values for compartment pressure, along with a summary of the relative difference for the tests.

For those tests in which the door to the compartment is open, the magnitude of the pressures are only a few Pascals; however, when the door is closed, the over-pressures are several hundred Pascals. For both the open- and closed-door tests, CFAST predicts the pressure to within experimental uncertainty with exceptions. The most notable exception is Test 16, which involved a large (2.3 MW) fire with the door closed and the ventilation on. By contrast, Test 10 involved a $1.2 \mathrm{MW}$ fire with comparable geometry and ventilation. There is considerable uncertainty in the magnitude of both the supply and return mass flow rates for Test 16. Compared to Test 16, Test 10 involves a greater measured supply velocity and a lesser measured exhaust velocity. This is probably the result of the higher pressure caused by the larger fire in Test 16. CFAST does not adjust the ventilation rate based on the compartment pressure until a specified cutoff pressure is reached. This is also the most likely explanation for the over-prediction of compartment pressure in Test 16.

In general, prediction of pressure in CFAST in closed compartments is critically dependent on correct specification of the leakage from the compartment. Compartments are rarely entirely sealed, and small changes in the leakage area can produce significant changes in the predicted over-pressure.

By comparison, the large relative differences for the two NBS compartment tests with furniture and wall-burning are qualitatively difference than the NIST/NRC outlier. For these two tests, the difference between model and experiment are on the order of $2 \mathrm{~Pa}$ rather than the more than $100 \mathrm{~Pa}$ of the NIST/NRC test. Qualitatively, the comparison between model and experiment for the NBS tests show similar curve shapes but with a notable single spike in the experimental measurements which particularly effects the comparison of peak values.

Based on the model physics and comparisons of model predictions with experimental measurements, CFAST calculations of pressure are seen as appropriate for the tests considered with the following reasons:

- With exceptions, CFAST predicts compartment pressures within experimental uncertainty.

- Prediction of compartment pressure for closed-door tests is critically dependent on correct specification of the leakage from the compartment. 

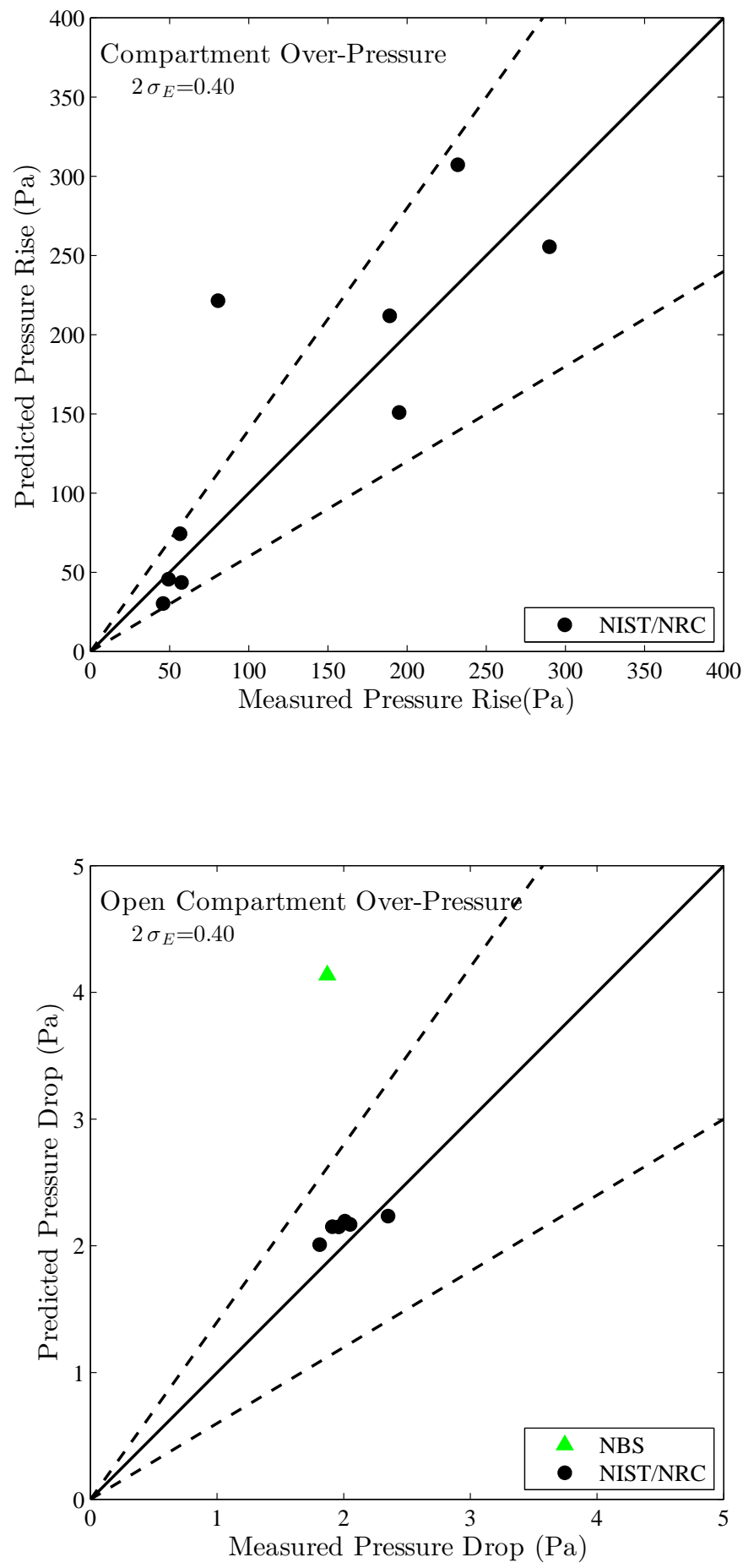

Figure 9.1: Comparison of Measured and Predicted Compartment Pressure. 


\section{Chapter 10}

\section{Heat Flux and Surface Temperature}

\subsection{NIST/NRC Test Series, Cables}

Target temperature and heat flux data are available from the NIST/NRC test series. In the NIST/NRC tests, the targets are different types of cables in various configurations: horizontal, vertical, in trays, or free-hanging. Figure 10.1 shows a comparison of predicted and measured values for radiation, total heat flux, and target temperature, along with a summary of the relative difference for the tests.

Appendix A provides nearly 200 comparisons of heat flux and surface temperature on four different cables. The following trends are notable comparing CFAST predictions to experimental measurements:

- Overall, the comparisons of target flux and temperature show larger relative differences than other quantities. This is to be expected since the target flux and temperature are inherently local quantities.

- The difference between predicted and measured cable surface temperatures is often within experimental uncertainty, with exceptions. Total target flux and target surface temperature predictions average within $2 \%$ of experimental values. However, there is considerable spread in the relative differences. Ignoring the sign of the relative difference, total target flux and target temperature average within $22 \%$ and $20 \%$, respectively. Accurate prediction of the surface temperature of the cable should indicate that the flux to the target (a combination of radiation from the fire, surrounding surfaces, and the gas layers, along with convection from the surrounding gas) should be correspondingly accurate. For the NIST/NRC tests, the cable surface temperature predictions show lower relative difference overall compared to the total heat flux and (particularly) the radiative heat flux.

- Total heat flux to targets is typically predicted to within an average difference of $2 \%$. Predictions for Cables D (horizontal) and G (vertical) are notable exceptions, with higher uncertainties.

- Radiative heat flux to targets is typically over-predicted compared to experimental measurements, with higher values for closed-door tests. For the closed-door tests, this may be a function of the over-prediction of the smoke concentration, which leads to the radiation contribution from the hot gas layer being a larger fraction of the total heat flux compared to the experimental values. 

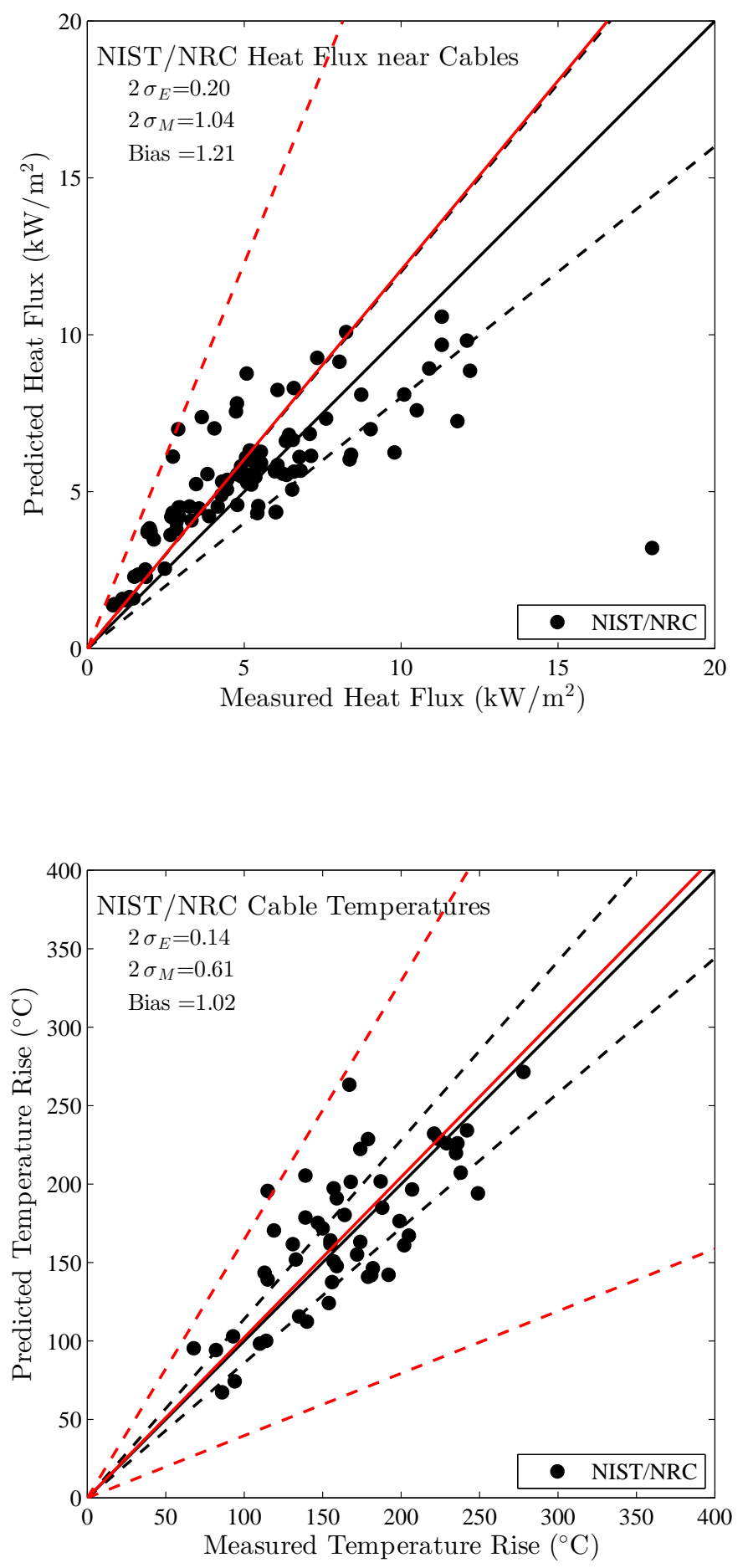

Figure 10.1: Comparisons of Measured and Predicted Heat Flux to Targets and Target Temperature 
- For many of the experiments, the convective heat flux component, taken to be the difference between the total heat flux and the radiative heat flux is seen to be higher than the values typically measured in fire experiments.

\subsection{NIST/NRC Test Series, Compartment Walls, Floor and Ceiling}

Thirty-six heat flux gauges were positioned at various locations on all four walls of the compartment, plus the ceiling and floor. Comparisons between measured and predicted heat fluxes and surface temperatures are shown on the following pages for a selected number of locations. Over half of the measurement points are in roughly the same relative location to the fire and hence the measurements and predictions are similar. For this reason, data for the east and north walls are shown because the data from the south and west walls are comparable. Data from the south wall is used in cases where the corresponding instrument on the north wall failed, or in cases where the fire is positioned close to the south wall. For each test, eight locations are used for comparison, two on the long (mainly north) wall, two on the short (east) wall, two on the floor, and two on the ceiling. Of the two locations for each panel, one is considered in the far-field, relatively remote from the fire; one is in the near-field, relatively close to the fire. How close or far varies from test to test, depending on the availability of working flux gauges. The two short wall locations are equally remote from the fire; thus, one location is in the lower layer, one in the upper.

Figure 10.1 shows a comparison of predicted and measured values for radiation, total heat flux for the tests.

CFAST predicts the heat flux and surface temperature of the compartment walls to within $121 \%$ and $21 \%$, respectively. The high value for heat flux is due to the way CFAST calculates flux to targets. The calculation of heat flux to a target in CFAST is done separately for the front face and rear face of the target. For target completely within a compartment, these two values are typically similar. For target on a compartment surface, the rear surface is assumed to radiate to ambient conditions. Thus, considering only the front face in the comparison should lead to a significant indicated overprediction of the heat flux. The predicted surface temperature is predicted far closer to experimental conditions that the surface heat flux indicating the actual heat flux is likely better than indicated by this front-face only comparison. Still, there is considerable scatter in the data indicating a higher uncertainty in prediction of heat flux and temperature to surfaces like those observed in heat flux and temperature to targets.

Typically, CFAST over-predicts the far-field fluxes and temperatures and under-predicts the near-field measurements. This is understandable, given that any two-zone model predicts an average representative value of gas temperature in the upper and lower regions of a compartment. Thus, the values predicted by CFAST should be an average of values near the fire and those farther away.

However, differences for the ceiling and (particularly) floor fluxes and temperatures are higher, with a more pronounced difference between the near-field and far-field comparisons. In addition to the limitations of the two-zone assumption, calculations of the flux to ceiling and floor surfaces are further confounded by the simple point-source calculation of radiation exchange in CFAST for the fire source. In CFAST, the fire is assumed to be a point source of energy located at the base 

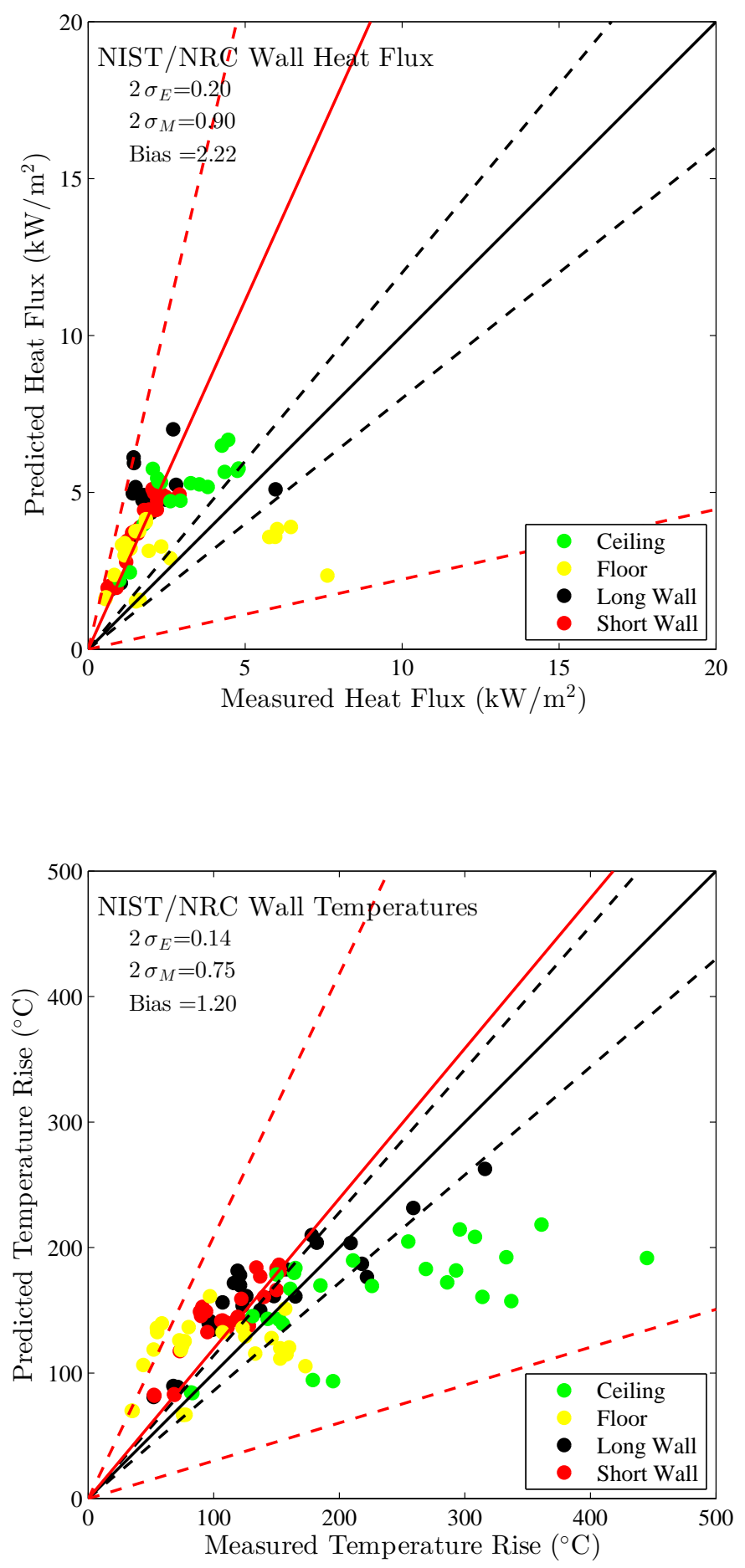

Figure 10.2: Comparisons of Measured and Predicted Heat Flux to Compartment Surfaces and Surface Temperature 
of the fire rather than a three-dimensional flame surface radiating to surroundings. With the fire typically at the floor surface, this makes the calculation of flux to the floor surface inherently less accurate than for other surfaces.

\subsection{Summary}

Use of CFAST for heat flux and temperature requires caution for the following reasons:

- Prediction of heat flux to targets and target surface temperature is largely dependent on local conditions surrounding the target. Like any two-zone model, CFAST predicts an average representative value of gas temperature in the upper and lower regions of a compartment. In addition, CFAST does not directly predict plume temperature or its effects on targets that may be within a fire plume. Thus, CFAST can be expected to under-predict values near a fire source, and over-predict values for targets remote from a fire.

- Cable target surface temperature predictions are often within experimental uncertainty, with exceptions, particularly for Cables F and G.

- Total heat flux to targets is typically predicted to within about $30 \%$.

- Radiative heat flux to targets is typically over-predicted compared to experimental measurements, with higher relative difference values for closed-door tests.

- CFAST is capable of predicting the surface temperature of a wall, assuming that its composition is fairly uniform and its thermal properties are well-characterized. Predictions are typically within $10 \%$ to $30 \%$. Generally, CFAST over-predicts the far-field fluxes and temperatures, and under-predicts the near-field measurements. This is consistent with the single representative layer temperature assumed by zone fire models.

- CFAST predictions of floor heat flux and temperature are more problematic because of the simple point-source calculation of radiative exchange between the fire and compartment surfaces. 


\section{Chapter 11}

\section{Summary and Conclusions}

How to best quantify the comparisons between model predictions and experiments is not obvious. The necessary and perceived level of agreement for any variable is dependent upon both the typical use of the variable in a given simulation, the nature of the experiment, and the context of the comparison in relation to other comparisons being made. For instance, the user may be interested in the time it takes to reach a certain temperature in the room, but have little or no interest in peak temperature for experiments that quickly reach a steady-state value. Insufficient experimental data and understanding of how to compare the numerous variables in a complex fire model prevent a complete validation of the model.

A true validation of a model would involve proper statistical treatment of all the inputs and outputs of the model with appropriate experimental data to allow comparisons over the full range of the model. Thus, the comparisons of the differences between model predictions and experimental data discussed here are intentionally simple and vary from test to test and from variable to variable due to the changing nature of the tests and typical use of different variables.

Table 11.1 summarizes the comparisons in this report.

For five of the quantities, the physics of the model is appropriate to represent the experimental conditions, and the calculated relative differences comparing the model and the experimental values are consistent with the combined experimental and input uncertainty. A few notes on the comparisons are appropriate:

- The CFAST predictions of the HGL temperature and height are, with a few exceptions, within or close to experimental uncertainty. The CFAST predictions are typical of those found in other studies where the HGL temperature is typically somewhat over-predicted and HGL height somewhat lower (HGL depth somewhat thicker) than experimental measurements. Still, predictions are mostly within $10 \%$ to $20 \%$ of experimental measurements. Calculation of HGL temperature and height has higher uncertainty in rooms remote from the fire (compared to those in the fire compartment).

- For most of the comparisons, CFAST predicts ceiling jet temperature well within experimental uncertainty. For cases where the HGL temperature is below $70{ }^{\circ} \mathrm{C}$, significant and consistent over-prediction was observed.

- CFAST predicts the flame height consistent with visual observations of flame height for the experiments. This is not surprising, given that CFAST simply uses a well-characterized 
Table 11.1: Summary of Model Comparisons

\begin{tabular}{|c|c|c|c|c|}
\hline Quantity & $\begin{array}{c}\text { Average } \\
\text { Difference }^{a} \\
(\%)\end{array}$ & $\begin{array}{c}\text { Median } \\
\text { Difference }^{b} \\
(\%)\end{array}$ & $\begin{array}{c}\text { Within } \\
\text { Experimental } \\
\text { Uncertainty }^{c} \\
(\%)\end{array}$ & $\begin{array}{c}\text { 90th } \\
\text { Percentile }^{d} \\
(\%)\end{array}$ \\
\hline HGL Temperature & 2 & 11 & 56 & 31 \\
\hline HGL Depth & -1 & 14 & 38 & 38 \\
\hline Plume Temperature & 30 & 29 & 15 & 43 \\
\hline Ceiling Jet Temperature & 4 & 4 & 89 & 17 \\
\hline Oxygen Concentration & 7 & 18 & 24 & 48 \\
\hline Carbon Dioxide Concentration & -12 & 14 & 24 & 35 \\
\hline Smoke Obscuration $^{e}$ & $278 / 24$ & $242 / 20$ & $0 / 81$ & $501 / 42$ \\
\hline Pressure & 35 & 13 & 82 & $163^{f}$ \\
\hline Target Flux (Total) & 2 & 17 & 58 & 46 \\
\hline Target Temperature & 2 & 19 & 41 & 43 \\
\hline Surface Flux (Total) ${ }^{g}$ & 121 & 121 & 0 & 174 \\
\hline Surface Temperature & 21 & 27 & 12 & 51 \\
\hline
\end{tabular}

a - average difference includes both the sign and magnitude of the relative differences in order to show any general trend to over- or under-prediction.

b - median difference is based only on the magnitude of the relative differences and ignores the sign of the relative differences so that values with opposing signs do not cancel and make the comparison appear closer than individual magnitudes would indicate.

$\mathrm{c}$ - the percentage of model predictions that are within experimental uncertainty.

$\mathrm{d}-90 \%$ of the model predictions are within the stated percentage of experimental values. For reference, a difference of $100 \%$ is a factor of 2 larger or smaller than experimental values.

e - the first number is for the closed door NIST/NRC tests and the second number if for the open door NIST/NRC tests.

f - high magnitude of the 90th percentile value driven in large part by two tests where underprediction was approximately $2 \mathrm{~Pa}$. If these two tests are not included, the 90th percentile value drops to $33 \%$.

$\mathrm{g}$ - reflects only flux to the front surface of the target. CFAST calculates flux to the front surface and rear surface separately. For surface targets, the rear surface is assumed at ambient temperature so this comparison to only the front surface is expected to significantly overestimate the actual flux to the entire target. 
experimental correlation to calculate flame height.

- Gas concentrations are typically under-predicted by CFAST, with an average difference of $-6 \%$ for oxygen concentration and $-16 \%$ for carbon dioxide concentration.

- Compartment pressure predicted by CFAST are within or close to experimental uncertainty for most tests.

Four of the quantities were seen to require additional care when using the model to evaluate the given quantity. This typically indicates limitations in the use of the model. A few notes on the comparisons are appropriate:

- CFAST typically predicts plume temperature near to experimental uncertainty, but tends to under-predict temperatures nearer to the fire source and over-predict temperatures farther away.

- CFAST typically over-predicts smoke concentration. Predicted concentrations for open-door tests are within experimental uncertainties, but those for closed-door tests are far higher.

- With exceptions, CFAST predicts cable surface temperatures within experimental uncertainties. Total heat flux to targets is typically predicted to within about $30 \%$, and often underpredicted. Radiative heat flux to targets is typically over-predicted compared to experimental measurements, with higher relative difference values for closed-door tests. Care should be taken in predicting localized conditions (such as target temperature and heat flux) because of inherent limitations in all zone fire models.

- Predictions of compartment surface temperature and heat flux are typically within $10 \%$ to $30 \%$. Generally, CFAST over-predicts the far-field fluxes and temperatures and underpredicts the near-field measurements. This is consistent with the single representative layer temperature assumed by zone fire models.

CFAST predictions in this validation study were consistent with numerous earlier studies, which show that the use of the model is appropriate in a range of fire scenarios. The CFAST model has been subjected to extensive evaluation studies by NIST and others. Although differences between the model and the experiments were evident in these studies, most differences can be explained by limitations of the model as well as of the experiments. Like all predictive models, the best predictions come with a clear understanding of the limitations of the model and the inputs provided to perform the calculations. 


\section{References}

[1] American Society for Testing and Materials, West Conshohocken, Pennsylvania. ASTM E 1355-04, Standard Guide for Evaluating the Predictive Capabilities of Deterministic Fire Models, 2004.

[2] R. D. Peacock, G. P. Forney, and P. A. Reneke. CFAST - Consolidated Model of Fire Growth and Smoke Transport (Version 6): Technical Reference Guide. Special Publication 1026, National Institute of Standards and Technology, Gaithersburg, Maryland, July 2011.

[3] EPRI/NRC-RES Fire PRA Methodology for Nuclear Power Facilities: Volume 2: Detailed Methodology. Technical Report EPRI TR-1011989 and NUREG/CR-6850, Electric Power Research Institute (EPRI), Palo Alto, CA, and U.S. Nuclear Regulatory Commission, Office of Nuclear Regulatory Research (RES), Rockville, MD, 2005.

[4] R. D. Peacock, W. W. Jones, P. A. Reneke, and G. P. Forney. CFAST - Consolidated Model of Fire Growth and Smoke Transport (Version 6): User's Guide. Special Publication 1041, National Institute of Standards and Technology, Gaithersburg, Maryland, December 2005.

[5] Verification and Validation of Selected Fire Models for Nuclear Power Plant Applications, Volume 5: Consolidated Fire and Smoke Transport Model (CFAST),. NUREG 1824, U. S. Nuclear Regulatory Commission, Office of Nuclear Regulatory Research, Rockville, MD, 2007.

[6] The Institute of Electrical and Electronics Engineers. IEEE Standard for Software Quality Assurance Plans, IEEE Std 730-2002 edition, 2002.

[7] Verification and Validation of Selected Fire Models for Nuclear Power Plant Applications, Volume 1: Main Report. NUREG 1824, U. S. Nuclear Regulatory Commission, Office of Nuclear Regulatory Research, Rockville, MD, 2007.

[8] K. E. Brenan, S. L. Campbell, and L. R. Petzold. Numerical Solution of Initial-Value Problems in Differential-Algebraic Equations. Elsevier Science Publishing, New York, 1989.

[9] G. P. Forney. Computing Radiative Heat Transfer Occurring in a Zone Fire Model. NISTIR 4709, National Institute of Standards and Technology, 1991.

[10] N. A. Dempsey, P. J. Pagni, and R. B. Williamson. Compartment Fire Experiments: Comparison With Models. Fire Safety Journal, 25(3):187, 1995. 
[11] International Conference of Building Officials, Whittier, CA. 1997 Uniform Building Code, 1997.

[12] W. M. Pitts, E. Braun, R. D. Peacock, H. E. Mitler, E. L. Johnsson, P. A. Reneke, and L. G. Blevins. Temperature Uncertainties for Bare-Bead and Aspirated Thermocouple Measurements in Fire Environments. In Thermal Measurements: The Foundation of Fire Standards, Special Technical Publication 1427, West Conshohocken, PA, 2001. American Society for Testing and Materials.

[13] Y. He and V. Beck. Smoke Spread Experiment in a Multi-Storey Building and Computer Modeling. Fire Safety Journal, 28(2):139, 1997.

[14] M. Luo, Y. He, and V. Beck. Comparison of Existing Fire Model Predictions With Experimental Results From Real Fire Scenarios. Journal of Applied Fire Science, 6(4):357, $1996 / 1997$.

[15] G. M. Poole, E. J. Weckman, and A. B. Strong. Fire Growth Rates in Structural Fires. NISTIR 5499, National Institute of Standards and Technology, 1994.

[16] J. Bailey and P. Tatem. Validation of Fire/Smoke Spread Model (CFAST) Using Ex-USS SHADWELL Internal Ship Conflagration Control (ISCC) Fire Tests. Technical Report NRL/MR/6180-95-7781, Naval Research Laboratory, 1995.

[17] J. L. Bailey, P. A. Tatem, W. W. Jones, and G. P. Forney. Development of an Algorithm to Predict Vertical Heat Transfer Trough Ceiling/Floor Conduction. Fire Technology, 34(2):139, 1998.

[18] S. Deal. A Review of Four Compartment Fires with Four Compartment Fire Models. In Proceedings of the Annual Meeting of the Fire Retardant Chemicals Association, volume Fire Safety Developments and Testing, pages 33-51, Ponte Verde Beach, Florida, October 21-24 1990. Fire Retardant Chemicals Association.

[19] L. Y. Cooper and G. P. Forney. The Consolidated Compartment Fire Model (CCFM) Computer Application CCFM-VENTS - Part I: Physical Reference Guide. NISTIR 4342, National Institute of Standards and Technology, 1990.

[20] H. E. Mitler and J. A. Rockett. Users' Guide to FIRST, A Comprehensive Single-Room Fire Model. NBSIR 87-3595, National Institute of Standards and Technology, September 1987.

[21] H. E. Nelson. FPETOOL: Fire Protection Engineering Tools for Hazard Estimation. NISTIR 4380, National Institute of Standards and Technology, 1990.

[22] W. W. Jones and R. D. Peacock. Technical Reference Guide for FAST Version 18. Technical Note 1262, National Institute of Standards and Technology, 1989.

[23] W. D. Davis. Comparison of Algorithms to Calculate Plume Centerline Temperature and Ceiling Jet Temperature With Experiments. Journal of Fire Protection Engineering, 12:9, 2002. 
[24] W. W. Jones and R. D. Peacock. Refinement and Experimental Verification of a Model for Fire Growth and Smoke Transport. In T. Wakamatsu, Y. Hasemi, A. Sekizawa, P. G. Seeger, P. J. Pagni, and C. E. Grant, editors, Fire Safety Science. Proceedings. 2nd International Symposium, pages 897-906, Tokyo, Japan, June 13-17 1988. International Association for Fire Safety Science, Hemisphere Publishing Corporation.

[25] R. S. Levine and H. E. Nelson. Full Scale Simulation of a Fatal Fire and Comparison of Results with Two Multiroom Models. NISTIR 90-4168, National Institute of Standards and Technology, 1990.

[26] D. Q. Duong. The Accuracy of Computer Fire Models: Some Comparisons with Experimental Data from Australia. Fire Safety Journal, 16:415, 1990.

[27] T. Tanaka. Model of Multiroom Fire Spread. Fire Science and Technology, 3(2):105-121, 1983.

[28] W. Chow. Predicability of Flashover by Zone Models. Journal of Fire Sciences, 16:335, September/October 1988.

[29] M. Luo, Y. He, and V. Beck. Application of Field Model and Two-Zone Model to Flashover Fires in a Full-Scale Multi-Room Single Level Building. Fire Safety Journal, 29:1, 1997.

[30] P. Collier. Fire in a Residential Building: Comparisons Between Experimental Data and a Fire Zone Model. Fire Technology, 32:195, August/September 1996.

[31] D. White, C. Beyler, J. Scheffrey, and F. Williams. Modeling the Impact of Post-Flashover Shipboard Fires on Adjacent Spaces. Journal of Fire Protection Engineering, 10, 2000.

[32] R. D. Peacock, P. A. Reneke, R. W. Bukowski, and V. Babrauskas. Defining Flashover for Fire Hazard Calculations. Fire Safety Journal, 32(4):331-345, 1999.

[33] V. Babrauskas, R. D. Peacock, and P. A. Reneke. Defining Flashover for Fire Hazard Calculations: Part II. Fire Safety Journal, 38:613-622, 2003.

[34] V. Babrauskas. Upholstered Furniture Room Fires-Measurements, Comparison with Furniture Calorimeter Data, and Flashover Predictions. Journal of Fire Sciences, 2(1):5-19, January/February 1984.

[35] S. Deal and C. Beyler. Correlating Preflashover Room Fire Temperatures. Journal of Fire Protection Engineering, 2(2):33-48, 1990.

[36] P. H. Thomas. Testing Products and Materials for Their Contribution to Flashover in Rooms. Fire and Materials, 5:103-111, 1981.

[37] B. J. McCaffrey, J. G. Quintiere, and M. F. Harkleroad. Estimating Room Temperatures and the Likelihood of Flashover Using Fire Test Data Correlations. Fire Technology, 17(2):98119, 1981.

[38] B. Hägglund. Estimating Flashover Potential in Residential Rooms. FOA Rapport C 202369A3, Forsvarets Forkningsanstalt, 1980. 
[39] W. W. Jones. Multicompartment Model for the Spread of Fire, Smoke and Toxic Gases. Fire Safety Journal, 9(1):172, 1985.

[40] J. A. Rockett and M. Morita. The NBS Harvard VI Multi-room Fire Simulation. Fire Science and Technology, 5(2):159-164, 1985.

[41] R. W. Bukowski. Modeling a Backdraft Incident: The 62 Watts Street (New York) Fire. Fire Engineers Journal, 56(185):14-17, 1996.

[42] W. Chow. Preliminary Studies of a Large Fire in Hong Kong. Journal of Applied Fire Science, 6(3):243-268, 1996/1997.

[43] R. W. Bukowski. Analysis of the Happyland Social Club Fire with HAZARD I. Fire and Arson Investigator, 42:36, 1992.

[44] J. Floyd. Comparison of CFAST and FDS for Fire Simulation With the HDR T51 and T52 Tests. NISTIR 6866, National Institute of Standards and Technology, 2002.

[45] K.B. McGrattan, S. Hostikka, J.E. Floyd, H.R. Baum, and R.G. Rehm. Fire Dynamics Simulator (Version 5), Technical Reference Guide. NIST Special Publication 1018-5, National Institute of Standards and Technology, Gaithersburg, Maryland, October 2007.

[46] G. Lui and W. Chow. A Short Note on Experimental Verification of Zone Models with an Electric Heater. International Journal on Engineering Performance-Based Fire Codes, 5:30, 2003.

[47] W. Chow. Studies on Closed Chamber Fires. Journal of Fire Sciences, 13:89, 1995.

[48] W. Chow. Experimental Evaluation of the Zone Models CFAST, FAST and CCFM.VENTS. Journal of Applied Fire Science, 2:307, 1992-1993.

[49] M. Altinakar, A. Weatherhill, and P. Nasch. Use of a Zone Model in Predicting Fire and Smoke Propagation in Tunnels. In J. R. Gillard, editor, 9th International Symposium on Aerodynamics and Ventilation of Vehicle Tunnels, pages 623-639, Aosta Valley, Italy, October 6-8 1997. BHR Group.

[50] R. D. Peacock, J. D. Averill, D. Madrzykowski, D. W. Stroup, P. A. Reneke, and R. W. Bukowski. Fire Safety of Passenger Trains; Phase III: Evaluation of Fire Hazard Analysis Using Full-Scale Passenger Rail Car Tests. NISTIR 6563, National Institute of Standards and Technology, 2004.

[51] J. Hoover and P. A. Tatem. Application of CFAST to Shipboard Fire Modeling. Part 3. Guidelines for Users. NRL/ML 6180-01-8550, Naval Research Laboratory, 2001.

[52] J. L. Bailey, G. P. Forney, P. A. Tatem, and W. W. Jones. Development and Validation of Corridor Flow Submodel for CFAST. Journal of Fire Protection Engineering, 12:139, 2002.

[53] W. Chow. Use of Zone Models on Simulating Compartmental Fires With Forced Ventilation. Fire and Materials, 14:466, 1995. 
[54] M. Luo. One Zone or Two Zones in the Room of Fire Origin During Fires? Journal of Fire Sciences, 15:240, 1997.

[55] D. Madrzykowski and R. Vettori. A Sprinkler Fire Suppression Algorithm for the GSA Engineering Fire Assessment System. NISTIR 4833, National Institute of Standards and Technology, 1992.

[56] W. Chow. Performance of Sprinkler in Atria. Journal of Fire Sciences, 14:466, 1996.

[57] K. Matsuyama, T. Wakamatsu, and K. Harada. Systematic Experiments of Room and Corridor Smoke Filling for Use in Calibration of Zone and CFD Fire Models. NISTIR 6588, National Institute of Standards and Technology, 2000.

[58] B. T. Lee. Effect of Wall and Room Surfaces on the Rates of Heat, Smoke, and Carbon Monoxide Production in a Park Lodging Bedroom Fire. NBSIR 85-2998, National Bureau of Standards, 1985.

[59] S. Hostikka, M. Kokkala, and J. Vaari. Experimental Study of the Localized Room Fires, NFDC2 Test Series. VTT Research Notes 2104, VTT Building and Transport, 2001.

[60] Verification and Validation of Selected Fire Models for Nuclear Power Plant Applications, Volume 2: Experimental Uncertainty. NUREG 1824, U. S. Nuclear Regulatory Commission, Office of Nuclear Regulatory Research, Rockville, MD, 2007.

[61] A. Hamins, M. Klassen, J. Gore, and T. Kashiwagi. Estimate of Flame Radiance via a Single Location Measurement in Liquid Pool Fires. Combustion and Flame, 86:223-228, 1991.

[62] A. Hamins, A. Maranghides, E. L. Johnsson, M. K. Donnelly, J. C. Yang, G. W. Mullholland, and R. L. Anleitner. Report of Experimental Results for the International Fire Model Benchmarking and Validation Exercise \#3. Special Publication 1013-1, National Institute of Standards and Technology, 2005.

[63] A. Hamins, A. Maranghides, and G. W. Mullholland. The Global Combustion Behavior of $1 \mathrm{MW}$ to $3 \mathrm{MW}$ Hydrocarbon Spray Fires Burning in an Open Environment. NISTIR 7013, National Institute of Standards and Technology, 2003.

[64] S. P. Nowlen. Enclosure Environment Characterization Testing for the Base line Validation of Computer Fire Simulation Codes. Technical Report NUREG/CR-4681, SAND86-1296, Sandia National Laboratories, Albuquerque, NM, 1987.

[65] A Summary of Nuclear Power Plant Fire Safety Research at Sandia National Laboratories 1975 - 1987. Technical report, Sandia National Laboratories, Albuquerque, NM, 1989.

[66] W. Klein-Helßling and M. Röwenkamp. Evaluation of Fire Models for Nuclear Power Plant Applications: Fuel Pool Fire Inside a Compartment. Technical report, Gesellschaft für Anlagen-und Reaktorsicherheit (GRS), Köln, Germany, May 2005.

[67] O. Riese and D. Hosser. Evaluation of Fire Model for Nuclear Power Plant Applications: Flame SPread in Cable Tray Fires. Draft Version Revision 1, Gesellschaft für Anlagen-und Reaktorsicherheit (GRS), June 2004. 
[68] R. D. Peacock, S. Davis, and B. T. Lee. Experimental Data Set for the Accuracy Assessment of Room Fire Models. NBSIR 88-3752, National Bureau of Standards, 1988.

[69] Fire Modeling Code Comparison. TR 108875, Electric Power Research Institute (EPRI), Palo Alto, CA.

[70] G. Heskestad and J. P. Hill. Experimental Fires in Multiroom/Corridor Enclosures. NBSGCR 86-502, National Institute of Standards and Technology, 1986.

[71] J. H. Klote. Fire Experiments of Zoned Smoke Control at the Plaza Hotel in Washington, DC. NISTIR 90-4253, National Institute of Standards and Technology, 1990.

[72] J. H. Klote and Jr. Fothergill, J. W. Design of Smoke Control Systems for Buildings. American Society of Heating, Refrigerating, and Air-Conditioning Engineers, Inc, 1983.

[73] J. H. Klote. Project Plan for Full Scale Smoke Movement and Smoke Control Tests. NBSIR 88-3800, National Bureau of Standards, 1988.

[74] J. E. Gott, D. L. Lowe, K. A. Notarianni, and W. D. Davis. Analysis of High Bay Hangar Facilities for Fire Detector Sensitivity and Placement. Technical Note 1423, National Institute of Standards and Technology, Gaithersburg, Maryland, February 1997.

[75] W. D. Davis, K. A. Notarianni, and P. Z. Tapper. An Algorithm for Calculating the Plume Centerline Temperature in the Presence of a Hot Upper Layer. Journal of Fire Protection Engineering, 10(3):23-31, 2000.

[76] M.L. Janssens and H.C. Tran. Data Reduction of Room Tests for Zone Model Validation. Journal of Fire Sciences, 10:528-555, 1992.

[77] K.B. McGrattan and G. P. Forney. Fire Dynamics Simulator (Version 4), User's Guide. NISTIR 7013, National Institute of Standards and Technology, June 2003.

[78] H.R. Baum and B. J. McCaffrey. Fire Induced Flow Field: Theory and Experiment. In T. Wakamatsu, Y. Hasemi, A. Sekizawa, and P. G. Seeger, editors, Fire Safety Science. Proceedings. 2nd International Symposium, pages 129-148, Tokyo, Japan, June 13-17 1989. International Association for Fire Safety Science, Hemisphere Publishing Corporation.

[79] B. J. McCaffrey. Momentum Implications for Buoyant Diffusion Flames. Combustion and Flame, 52:149, 1983.

[80] L. Y. Cooper. Fire-Plume-Generated Ceiling Jet Characteristics and Convective Heat Transfer to Ceiling and Wall Surfaces in a Two-Layer Zone-Type Fire Environment. NISTIR 4705, National Institute of Standards and Technology, 1991.

[81] M. Bundy, A. Hamins, E. L. Johnsson, C. K. Kim, G. H. Ko, and D. B. Lenhert. Measurements of Heat and Combustion Products in Reduced-Scale Ventilation-Limited Compartment Fires. Technical Note 1483, National Institute of Standards and Technology, July 2007. 
[82] Verification and Validation of Selected Fire Models for Nuclear Power Plant Applications, Volume 7: FDS. NUREG 1824, U. S. Nuclear Regulatory Commission, Office of Nuclear Regulatory Research, Rockville, MD, 2007.

[83] Y. He, A. Fernando, and M. Luo. Determination of Interface Height from Measured Parameter Profile in Encolsure Fire Experiment. Fire Safety Journal, 31:19-38, 1998. 


\section{Appendix A}

\section{Calculation of Layer Height and the Average Upper and Lower Layer Temperatures}

Fire protection engineers often need to estimate the location of the interface between the hot, smoke-laden upper layer and the cooler lower layer in a burning compartment. Zone fire models such as CFAST compute this quantity directly, along with the average temperature of the upper and lower layers. In an experimental test or a computational fluid dynamics (CFD) model like FDS [45], there are not two distinct zones, but rather a continuous profile of temperature. Nevertheless, methods have been developed to estimate layer height and average temperatures from a continuous vertical profile of temperature. One such method [76] is as follows: Consider a continuous function $T(z)$ defining temperature $T$ as a function of height above the floor $z$, where $z=0$ is the floor and $z=H$ is the ceiling. Define $T_{u}$ as the upper layer temperature, $T_{l}$ as the lower layer temperature, and $z_{\text {int }}$ as the interface height. Compute the quantities:

$$
\begin{aligned}
& \left(H-z_{\text {int }}\right) T_{u}+z_{\text {int }} T_{l}=\int_{0}^{H} T(z) d z=I_{1} \\
& \left(H-z_{\text {int }}\right) \frac{1}{T_{u}}+z_{\text {int }} \frac{1}{T_{l}}=\int_{0}^{H} \frac{1}{T(z)} d z=I_{2}
\end{aligned}
$$

Solve for $z_{\text {int }}$ :

$$
z_{\text {int }}=\frac{T_{l}\left(I_{1} I_{2}-H^{2}\right)}{I_{1}+I_{2} T_{l}^{2}-2 T_{l} H}
$$

Let $T_{l}$ be the temperature in the lowest mesh cell and, using Simpson's Rule, perform the numerical integration of $I_{1}$ and $I_{2} . T_{u}$ is defined as the average upper layer temperature via

$$
\left(H-z_{\text {int }}\right) T_{u}=\int_{z_{\text {int }}}^{H} T(z) d z
$$

For experimental test data or CFD model output, the integral function of temperature as a function of height can be estimated empirically from a number of discrete data points. Further discussion of similar procedures can be found in Ref. [83]. 


\section{Appendix B}

\section{Model / Experiment Comparison Graphs}

\section{B.1 NBS Single Room Tests with Furniture}

These data describe a series of room fire tests using upholstered furniture items in a room of fixed size but with varying opening sizes and shapes [34] conducted by the National Bureau of Standards (NBS, former name of NIST). It was selected for its well characterized and realistic fuel sources in a simple single-room geometry. In addition, the wide variation in opening size should provide challenges for current zone fire models. Peak fire size was about 2.9 MW with a total room volume of $21 \mathrm{~m}^{3}$. A series of four single-room fire tests were conducted using upholstered furniture items for comparison with their free burning behavior, previously determined in a furniture calorimeter. The experiments were conducted in a single room enclosure; ventilation to the room was provided by window openings of varying sizes. The room was equipped with an instrumented exhaust collection system outside the window opening.

A second similar test series also utilized a single-room fire test with furniture as the fire source [58]. It expanded upon the first data set by adding the phenomenon of wall burning. Peak fire size was about $7 \mathrm{MW}$. The room size was similar to the first test series. 

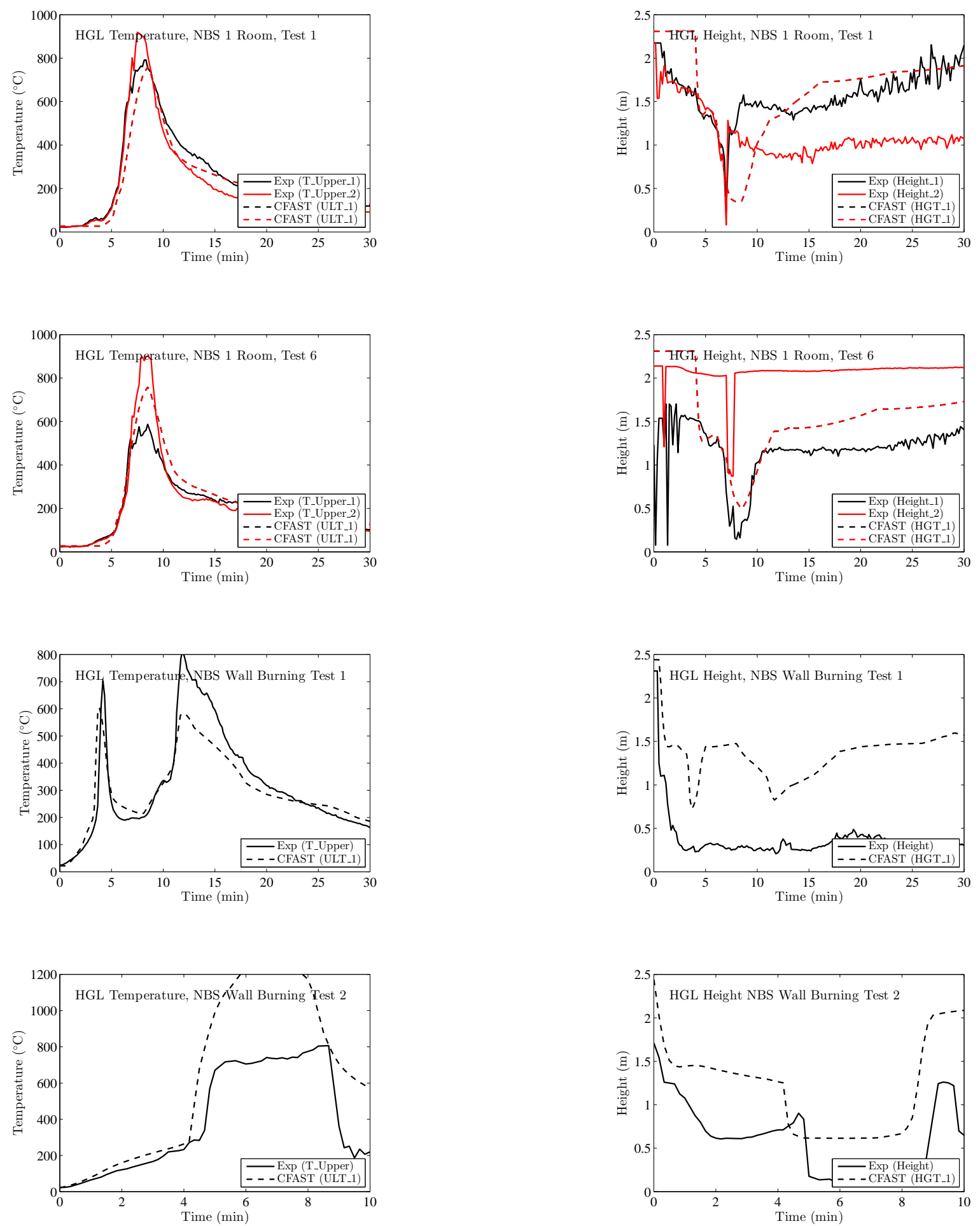

Figure B.1: Predicted HGL Temperature and Height for the NBS Single Compartment Tests. 

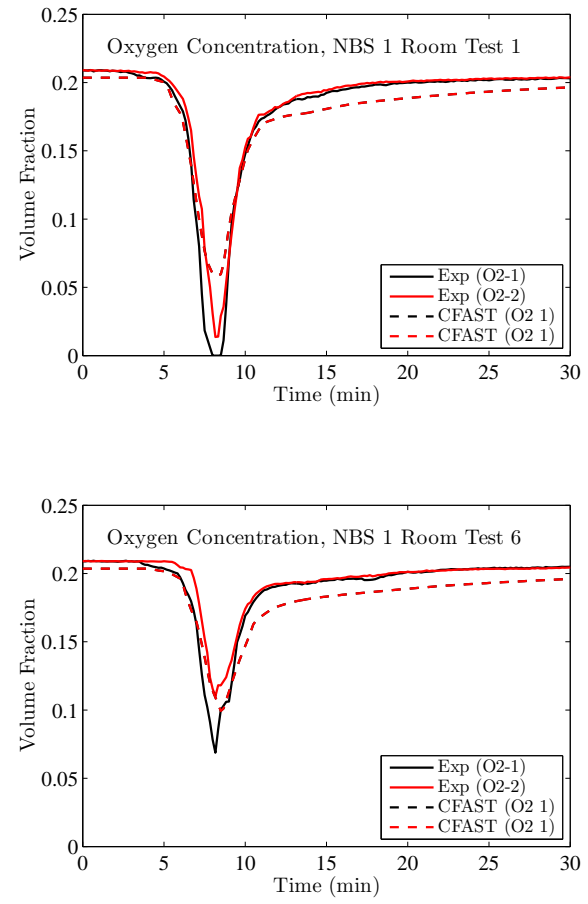

Figure B.2: Predicted Oxygen Concentrationfor the NBS Single Compartment Tests. 

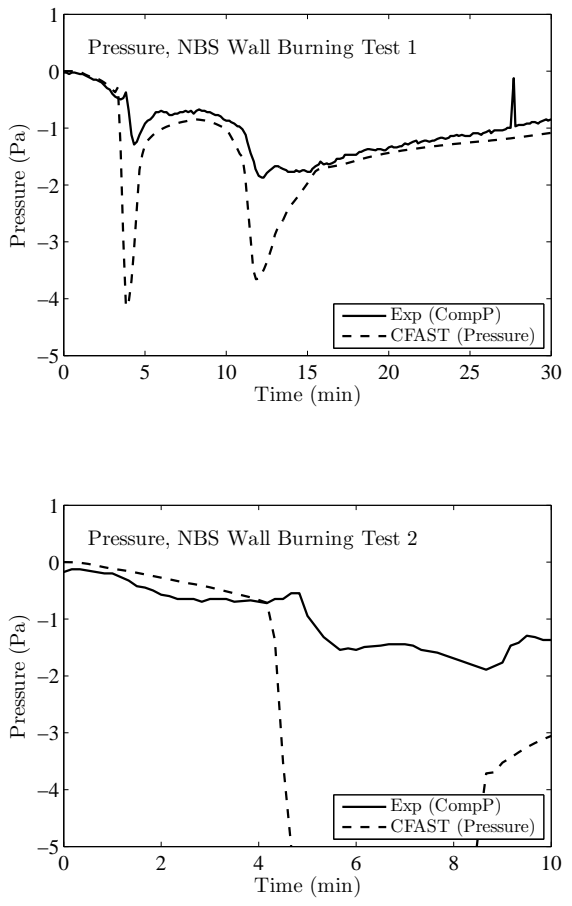

Figure B.3: Predicted Compartment Pressure for the NBS Single Compartment Tests. 


\section{B.2 VTT Large Hall Tests}

The experiments are described in reference [59]. The series consisted three unique fire scenarios with replications for a total of 8 experiments. The experiments were undertaken to study the movement of smoke in a large hall with a sloped ceiling. The tests were conducted inside the VTT Fire Test Hall, with dimensions of $19 \mathrm{~m}$ high by $27 \mathrm{~m}$ long by $14 \mathrm{~m}$ wide. Each test involved a single heptane pool fire, ranging from $2 \mathrm{MW}$ to $4 \mathrm{MW}$. 

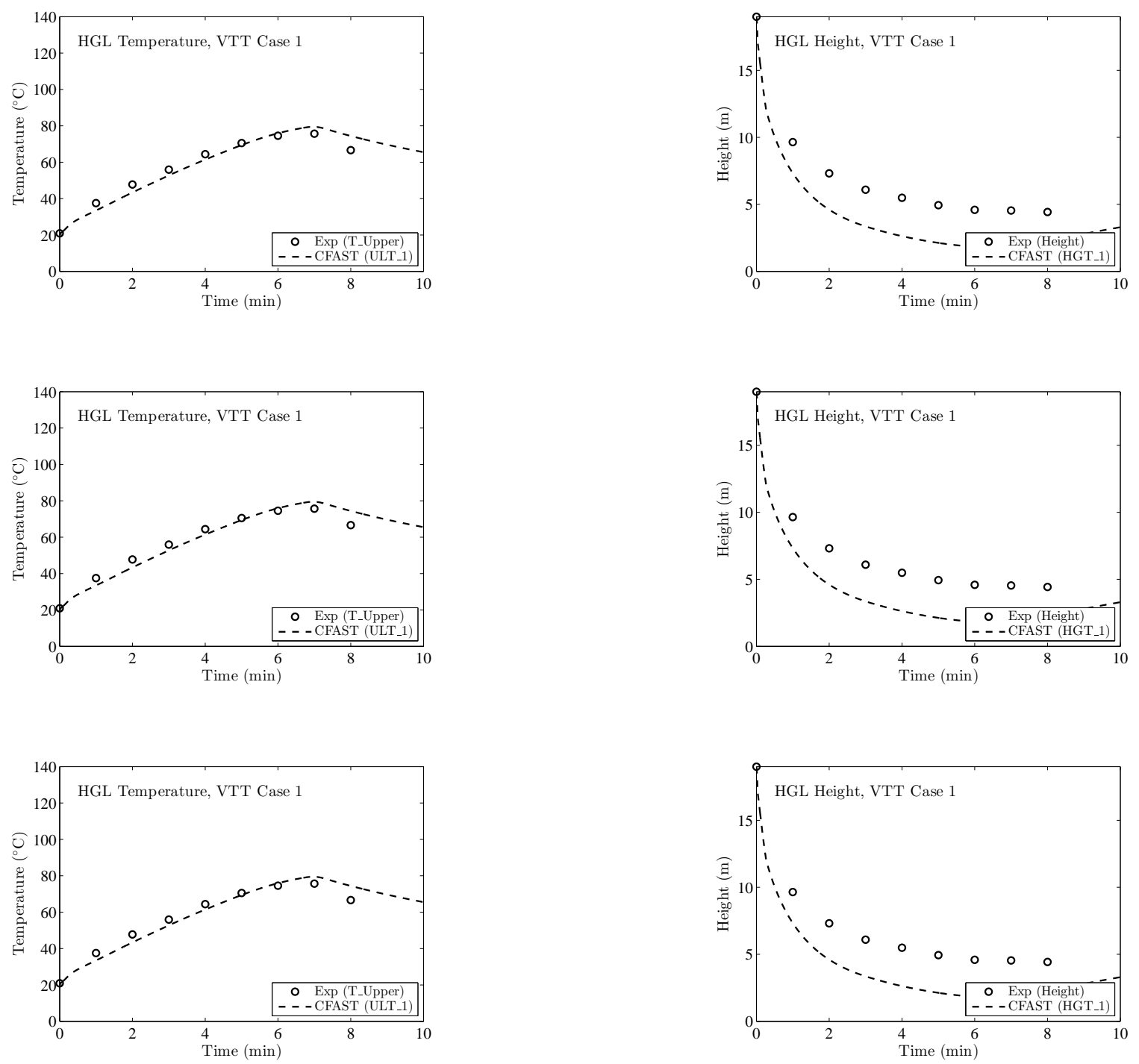

Figure B.4: Predicted HGL Temperature and Height for the VTT Large Hall Tests. 

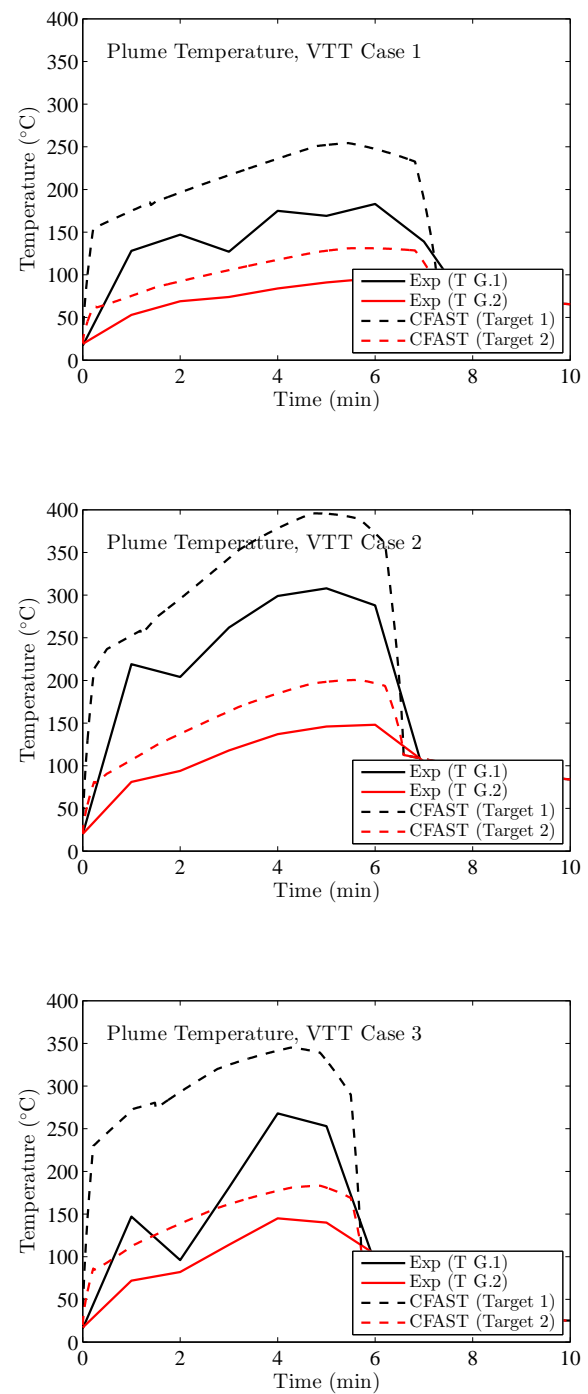

Figure B.5: Predicted Plume Centerline Temperature for the VTT Large Hall Tests. 


\section{B.3 NIST/NRC Test Series}

These experiments, sponsored by the US NRC and conducted at NIST, consisted of 15 largescale experiments performed in June 2003. All 15 tests were included in the validation study. The experiments are documented in Ref. [62]. The fire sizes ranged from $350 \mathrm{~kW}$ to $2.2 \mathrm{MW}$ in a compartment with dimensions $21.7 \mathrm{~m}$ by $7.1 \mathrm{~m}$ by $3.8 \mathrm{~m}$ high, designed to represent a compartment in a nuclear power plant containing power and control cables. The room had one door and a simple mechanical ventilation system. Ventilation conditions, the fire size, and fire location were varied. Numerous measurements (approximately 350 per test) were made. 

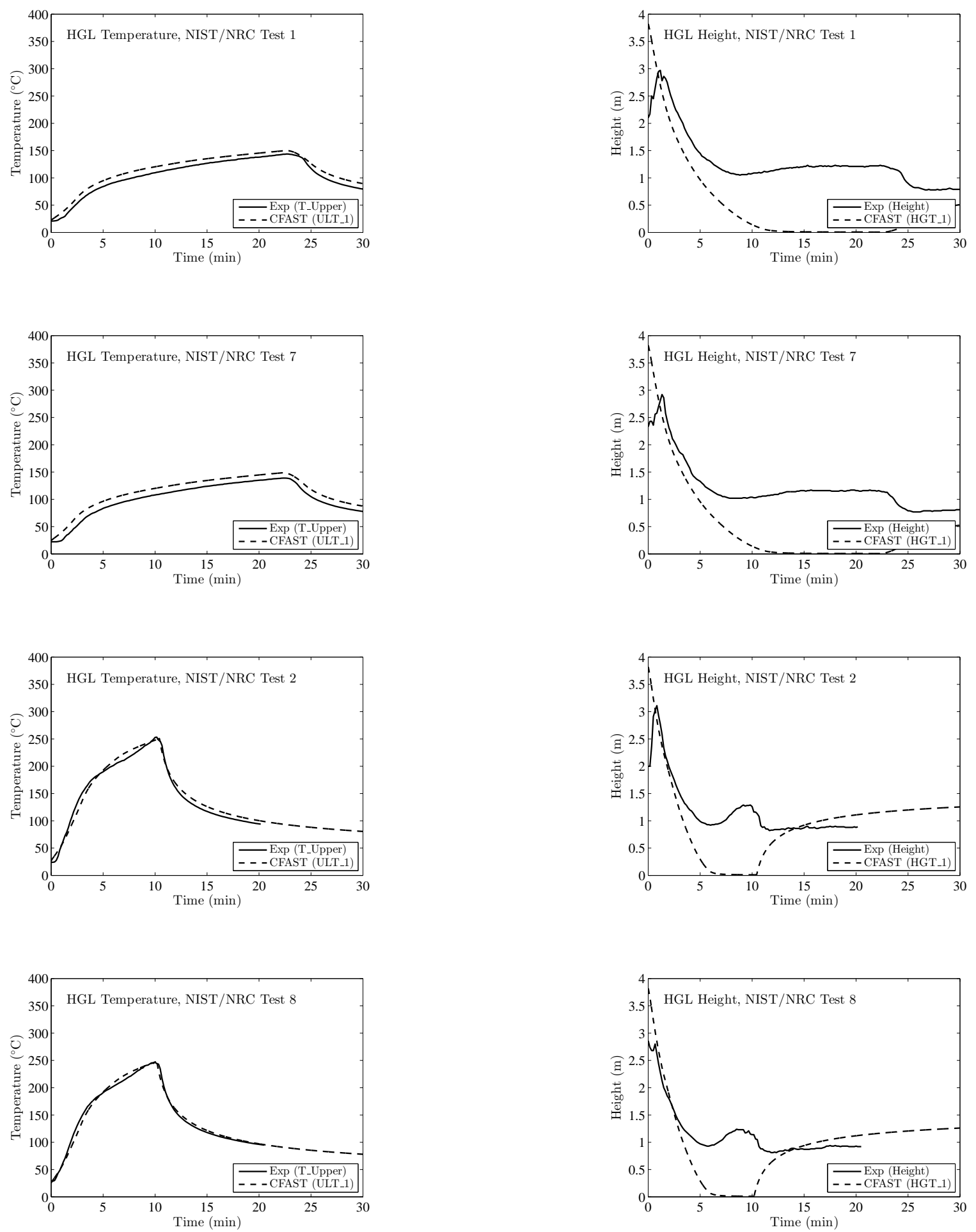

Figure B.6: Predicted HGL Temperature and Height for the NIST/NRC Tests 1, 7, 2 and 8. 

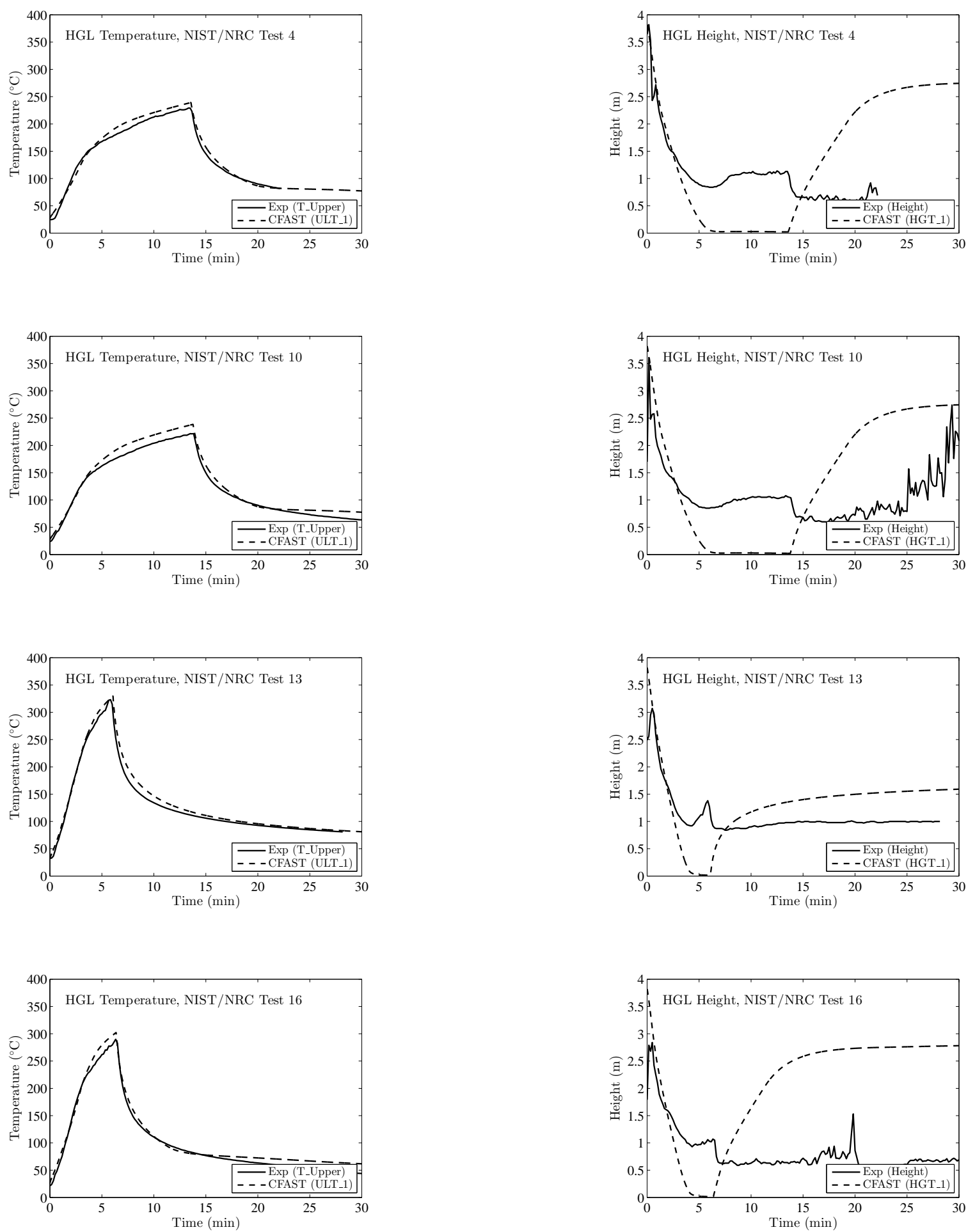

Figure B.7: Predicted HGL Temperature and Height for the NIST/NRC Tests 4, 10, 13 and 16. 

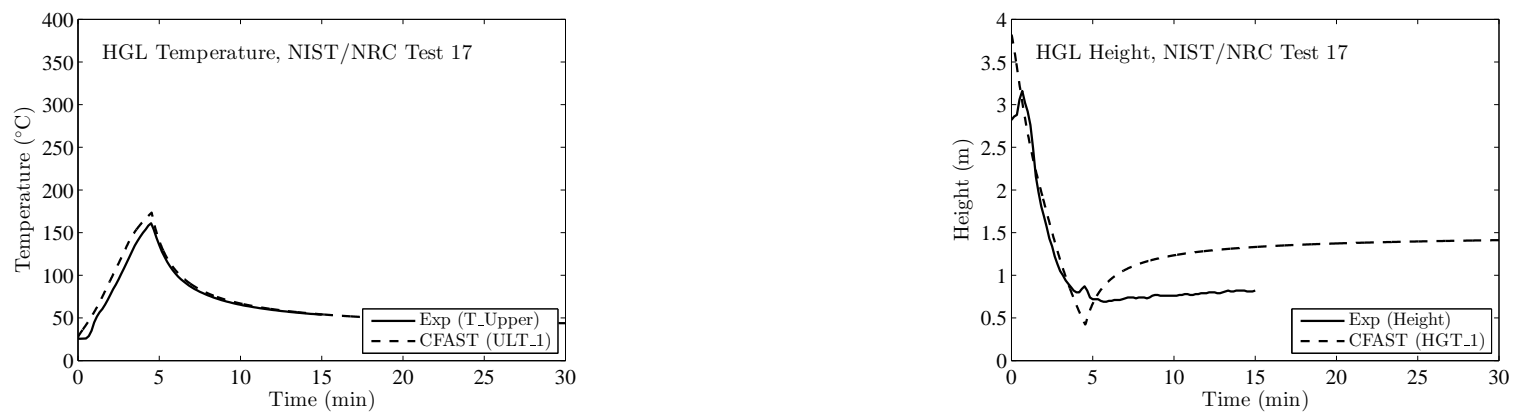

\section{Open Door Tests to follow}
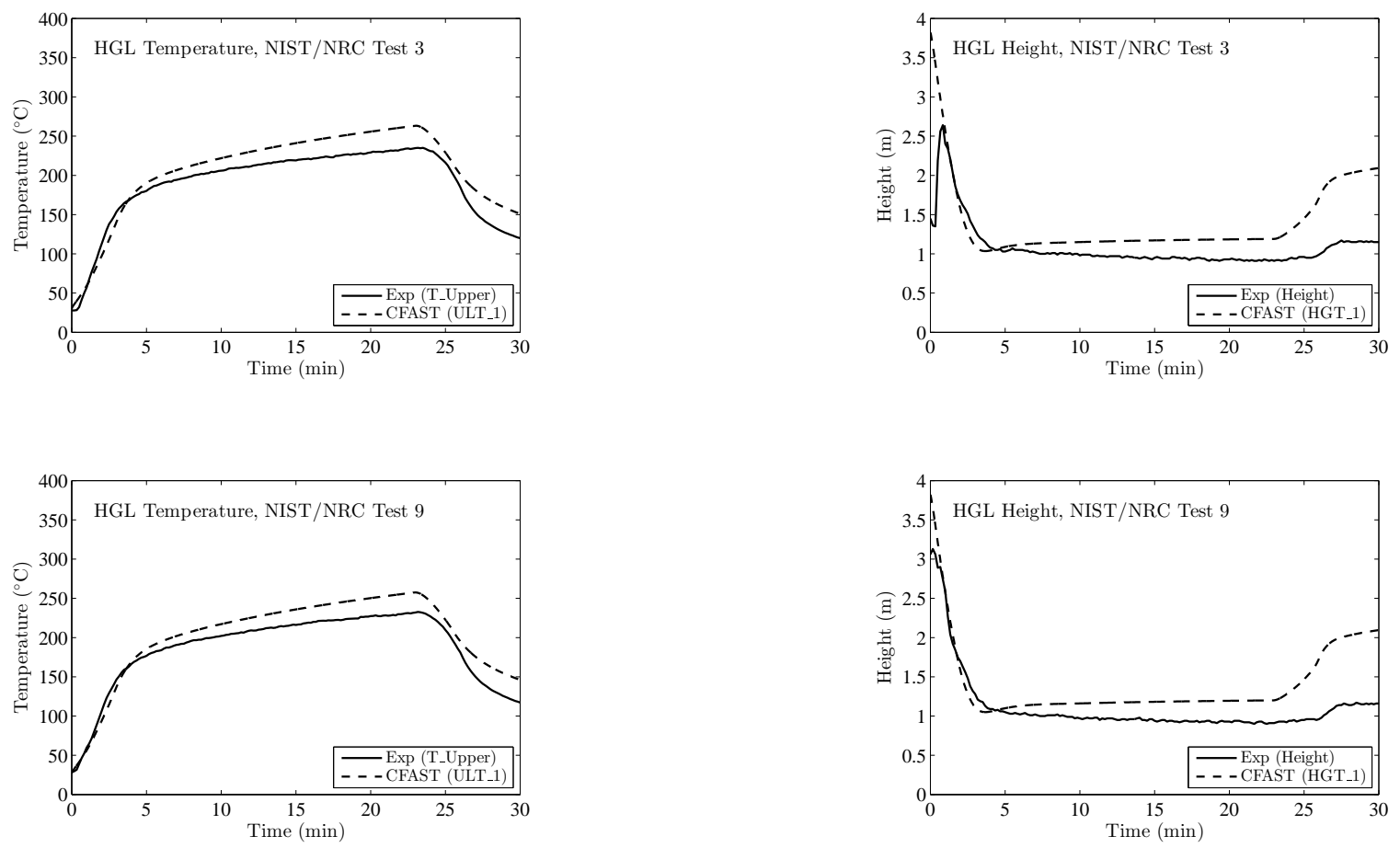

Figure B.8: Predicted HGL Temperature and Height for the NIST/NRC Tests 17, 3 and 9. 

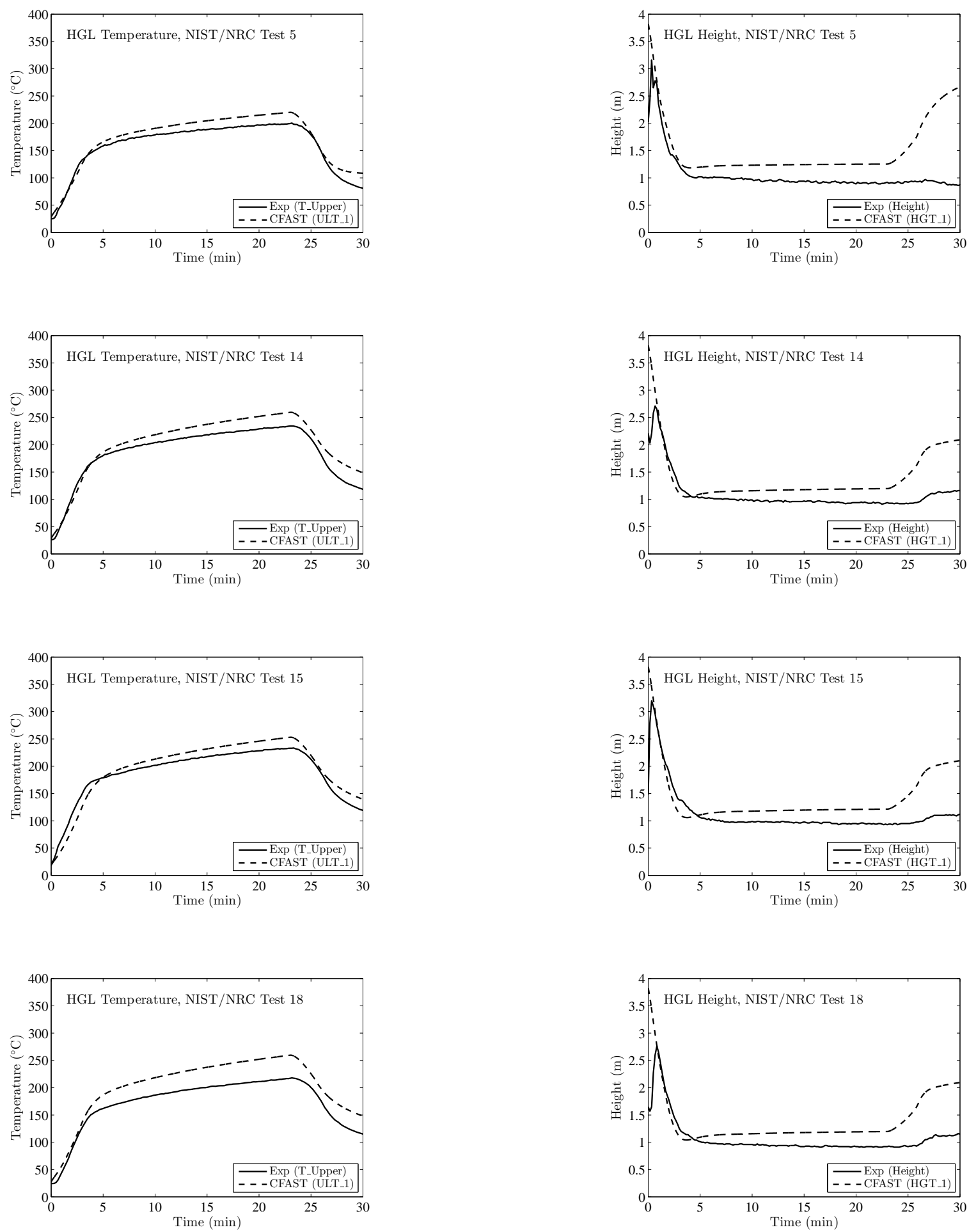

Figure B.9: Predicted HGL Temperature and Height for the NIST/NRC Tests 5, 14, 15 and 18. 

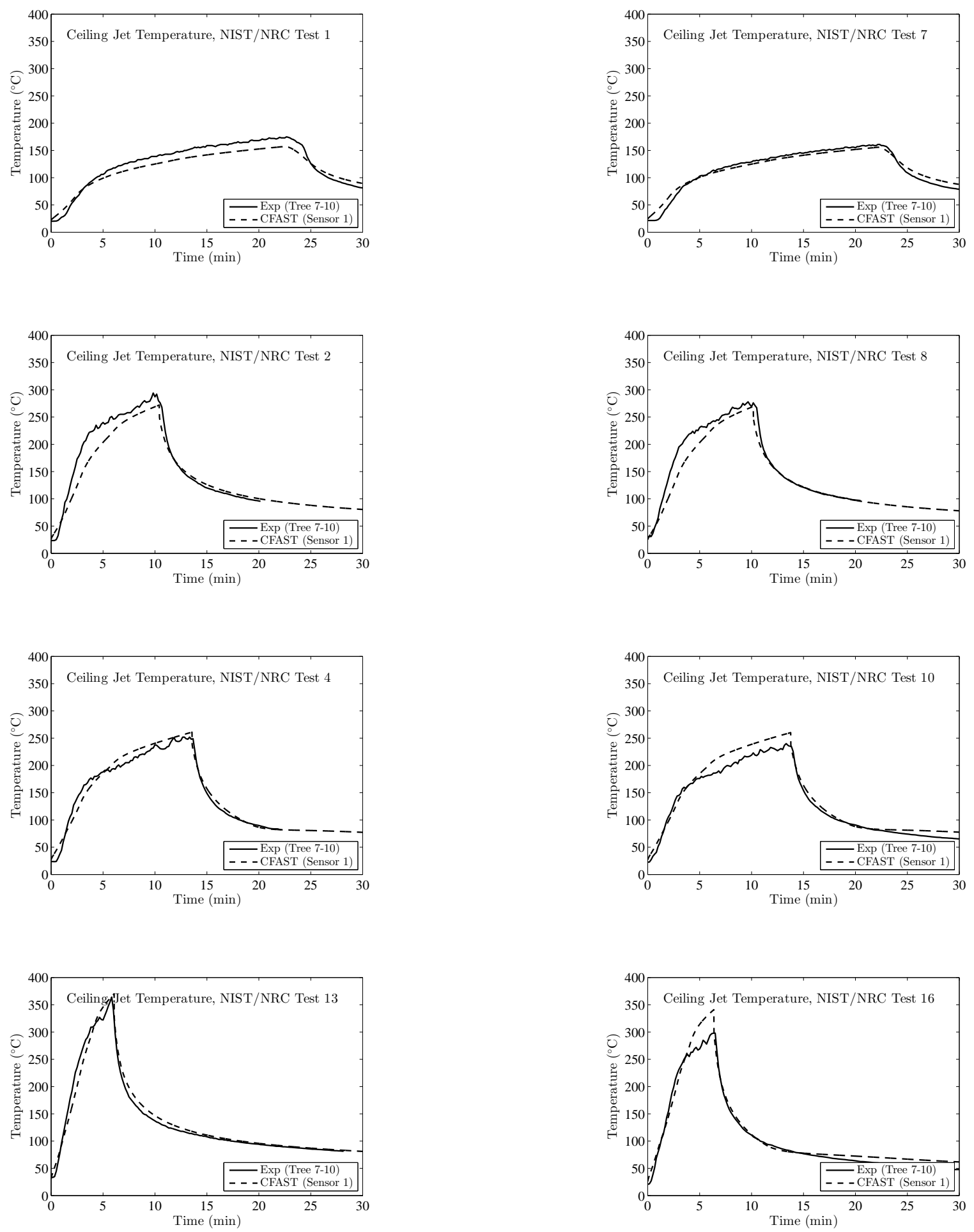

Figure B.10: Ceiling Jet Temperature for the NIST/NRC Series, Closed Door Tests. 

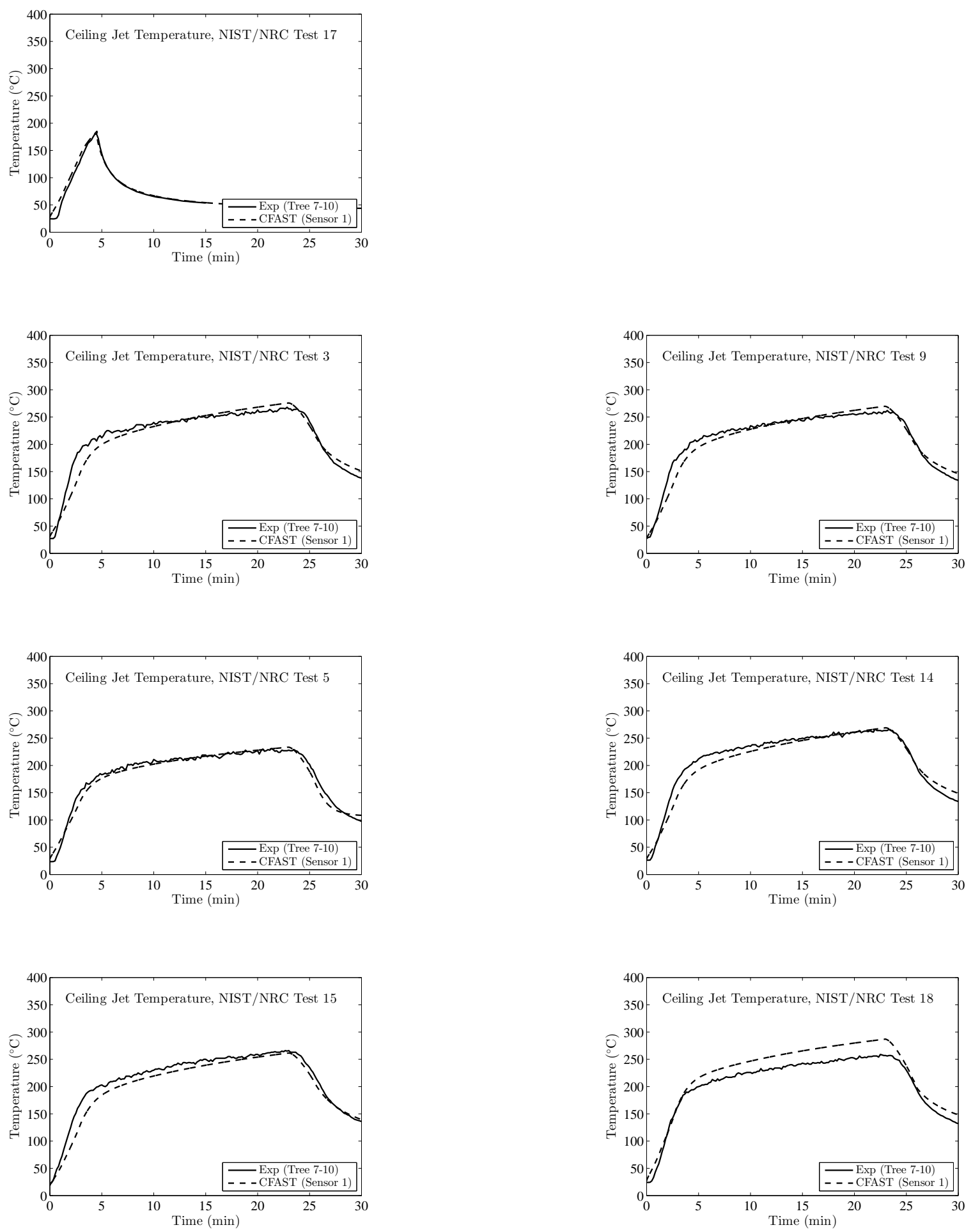

Figure B.11: Ceiling Jet Temperature for the NIST/NRC Series, Open Door Tests. 

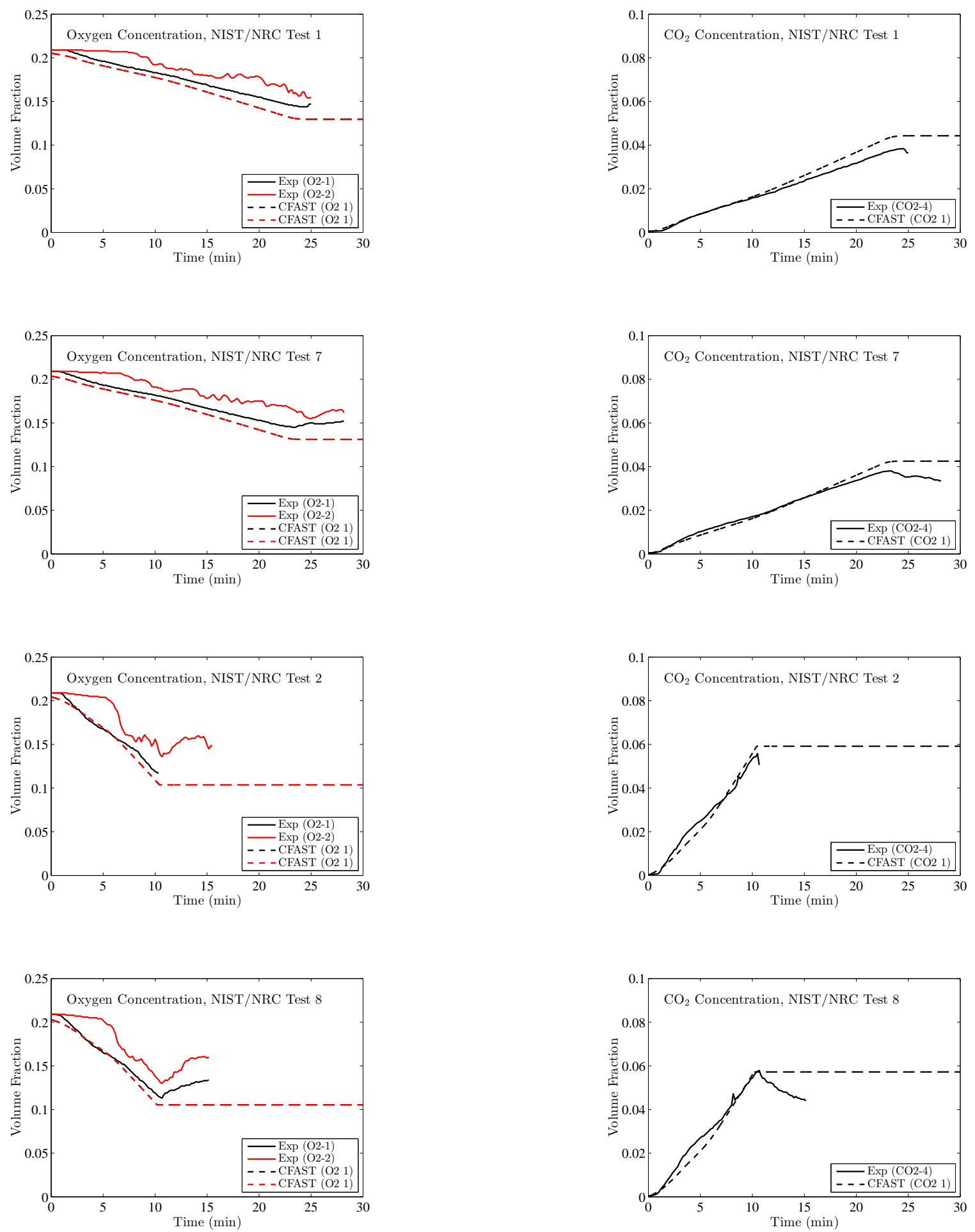

Figure B.12: Predicted Oxygen and Carbon Dioxide for the NIST/NRC Tests 1, 7, 2 and 8. 

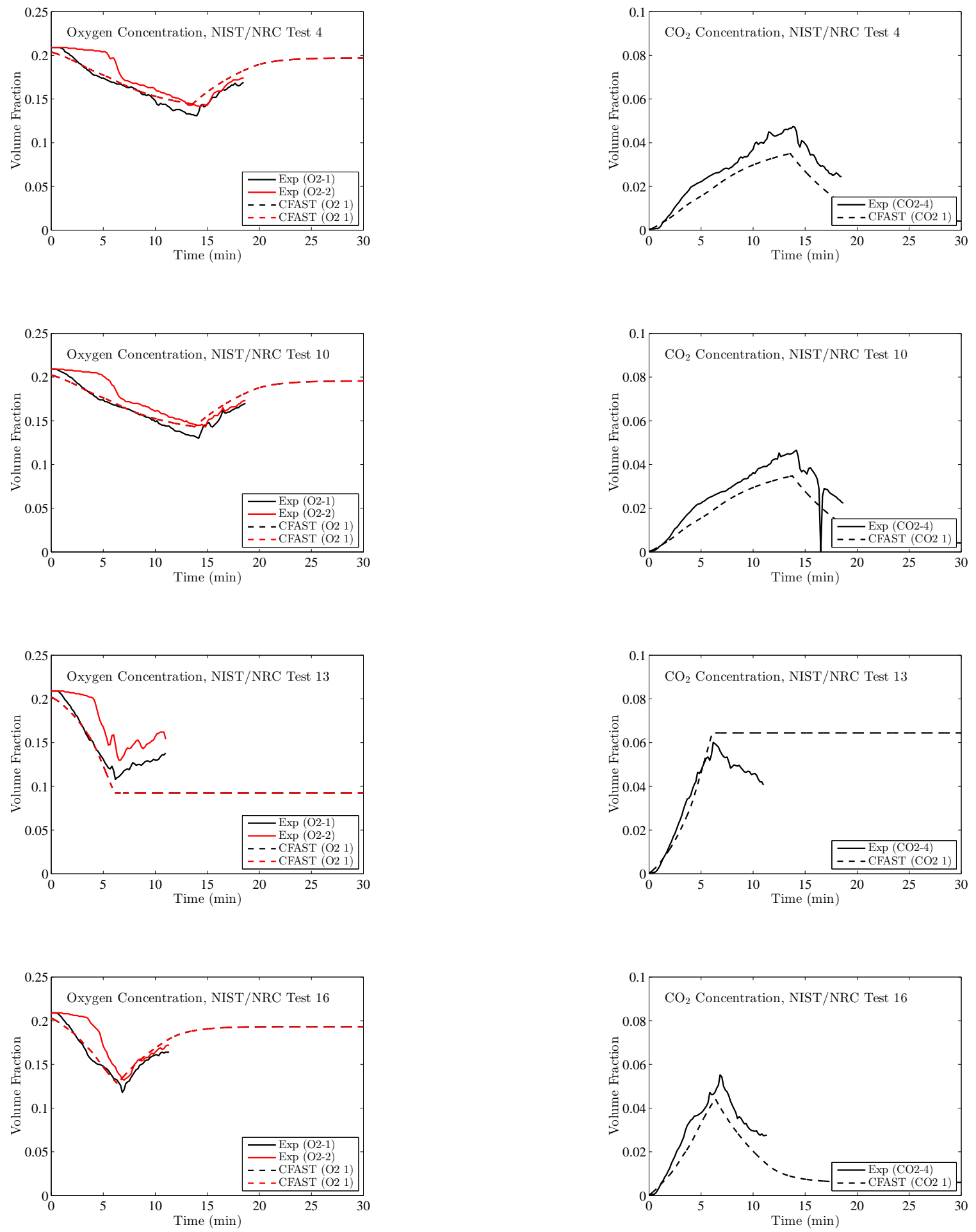

Figure B.13: Predicted Oxygen and Carbon Dioxide for the NIST/NRC Tests 4, 10, 13 and 16. 

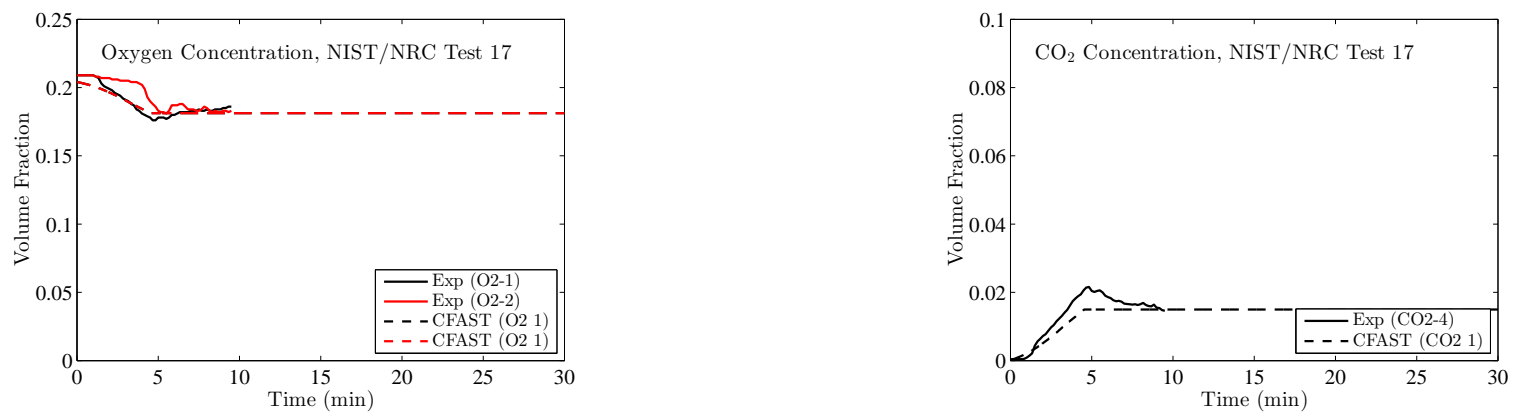

\section{Open Door Tests to follow}
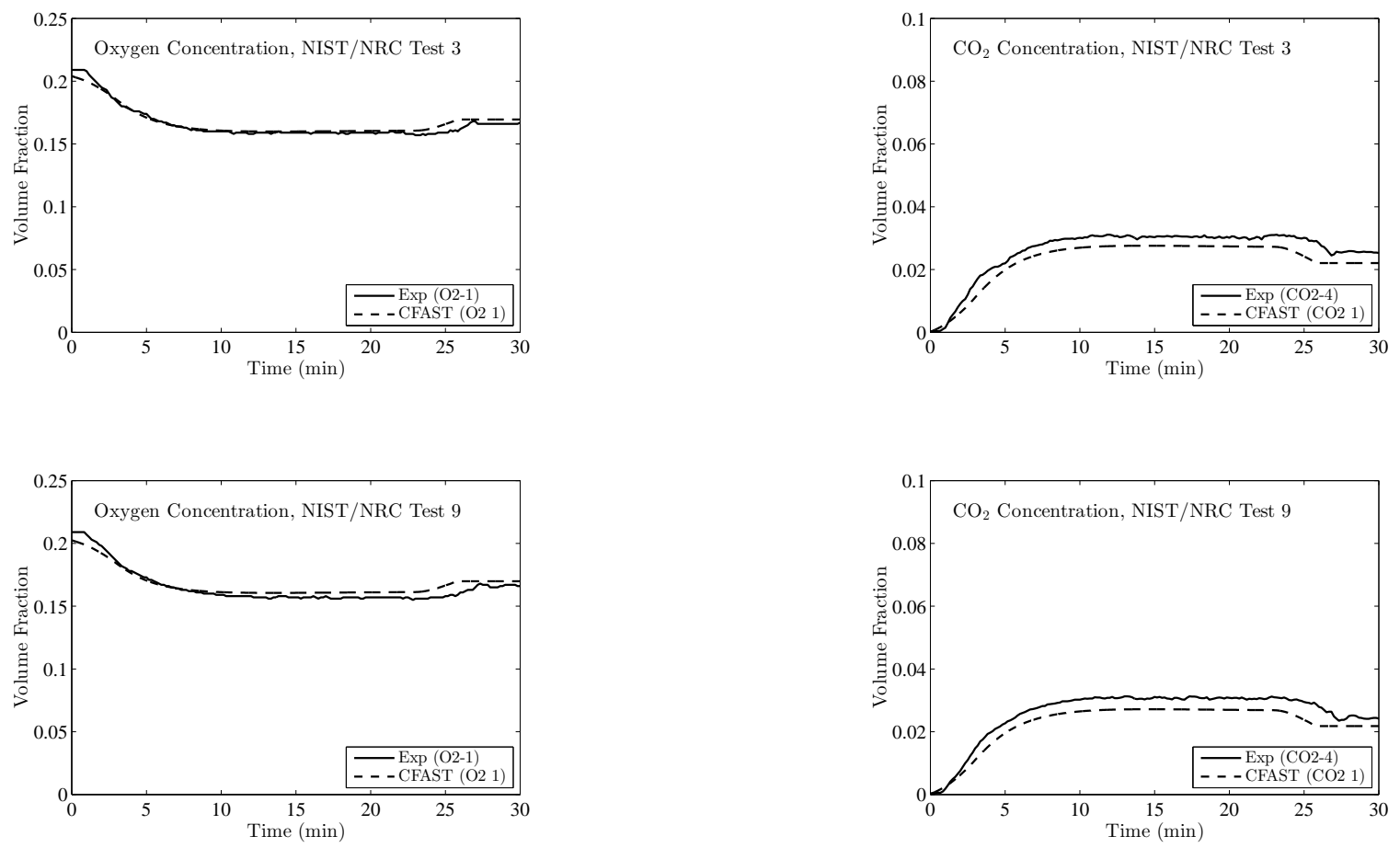

Figure B.14: Predicted Oxygen and Carbon Dioxide for the NIST/NRC Tests 17, 3, and 9. 

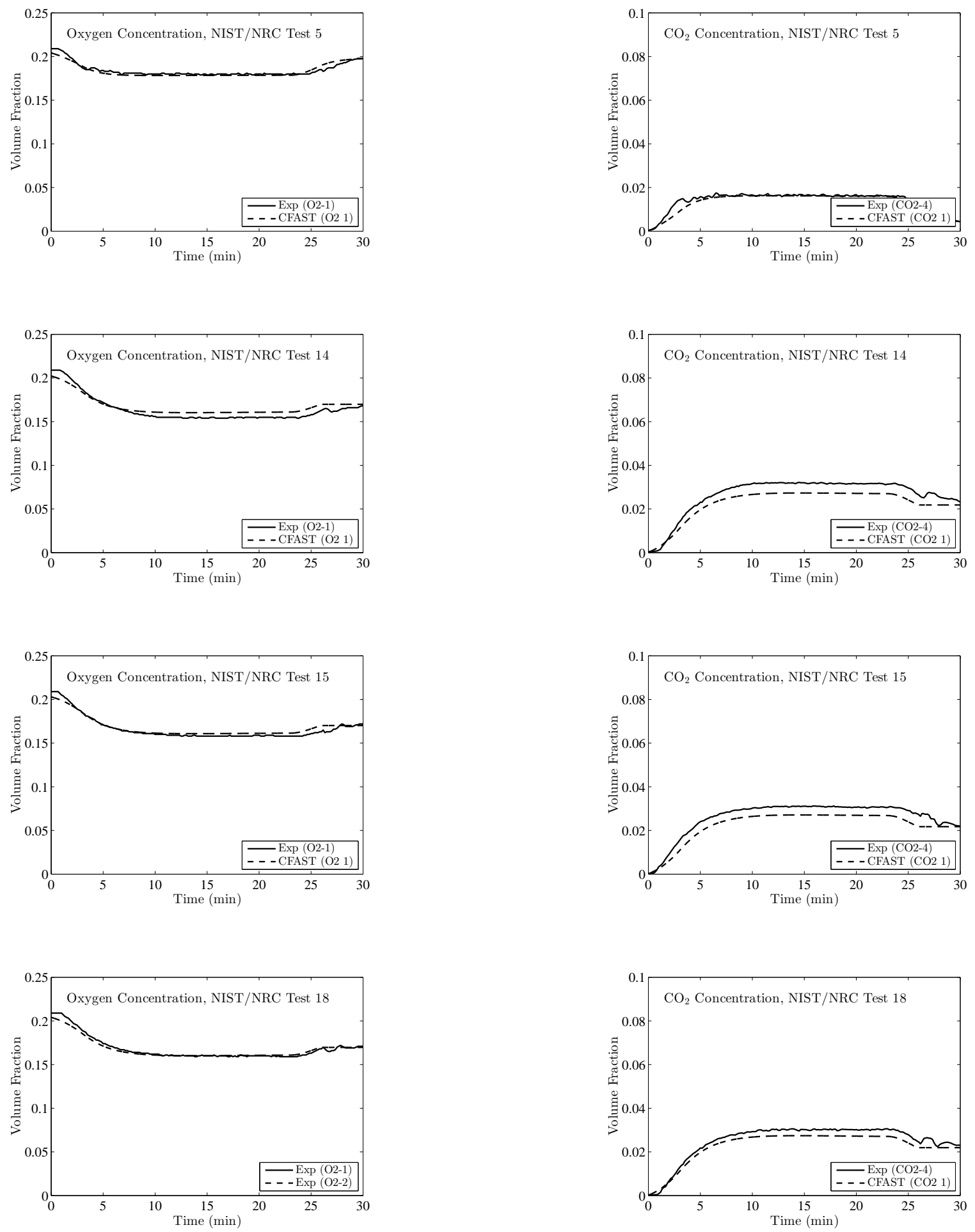

Figure B.15: Predicted Oxygen and Carbon Dioxide for the NIST/NRC Tests 5, 14, 15 and 18. 

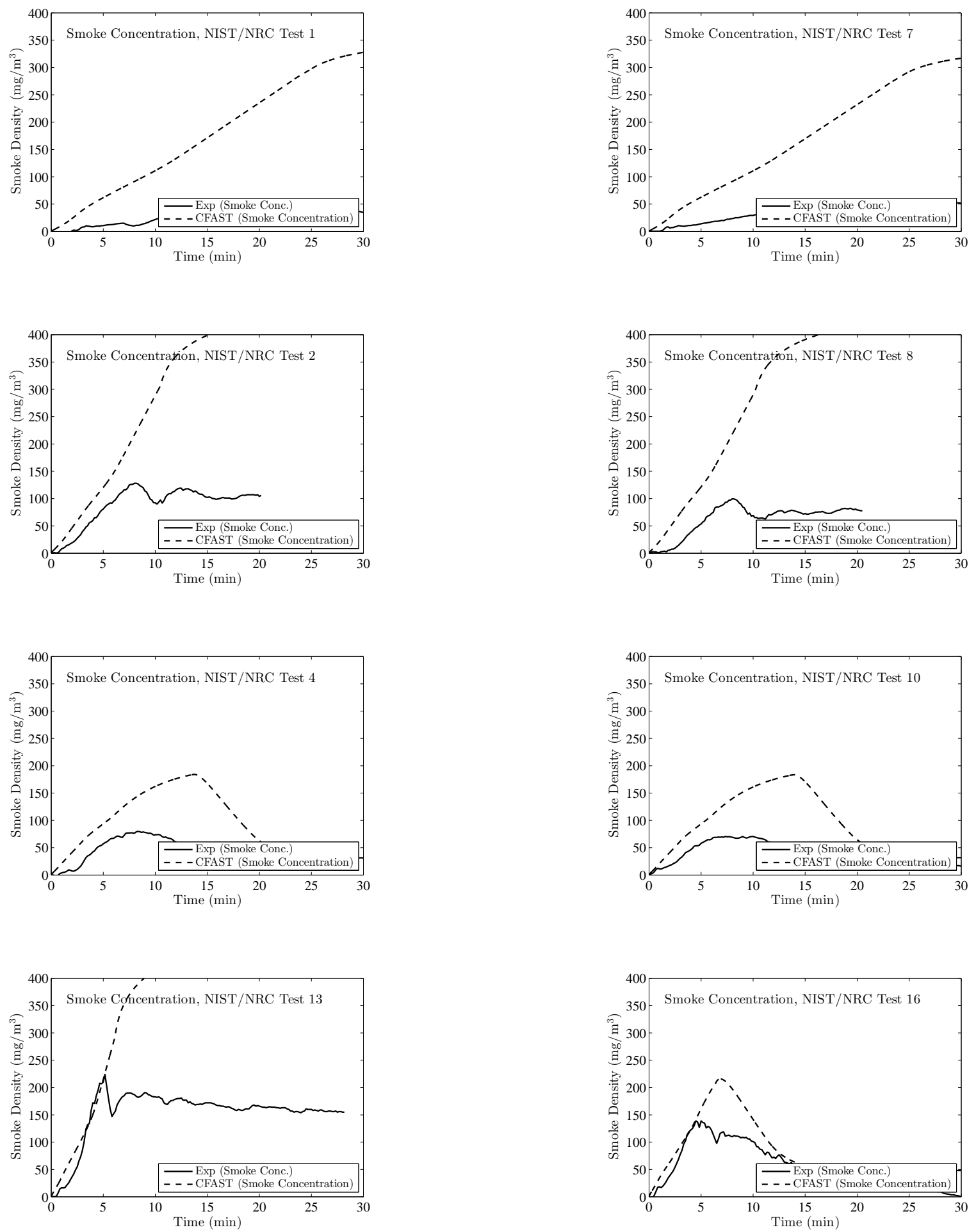

Figure B.16: Smoke Concentrationfor the NIST/NRC Series, Closed Door Tests. 

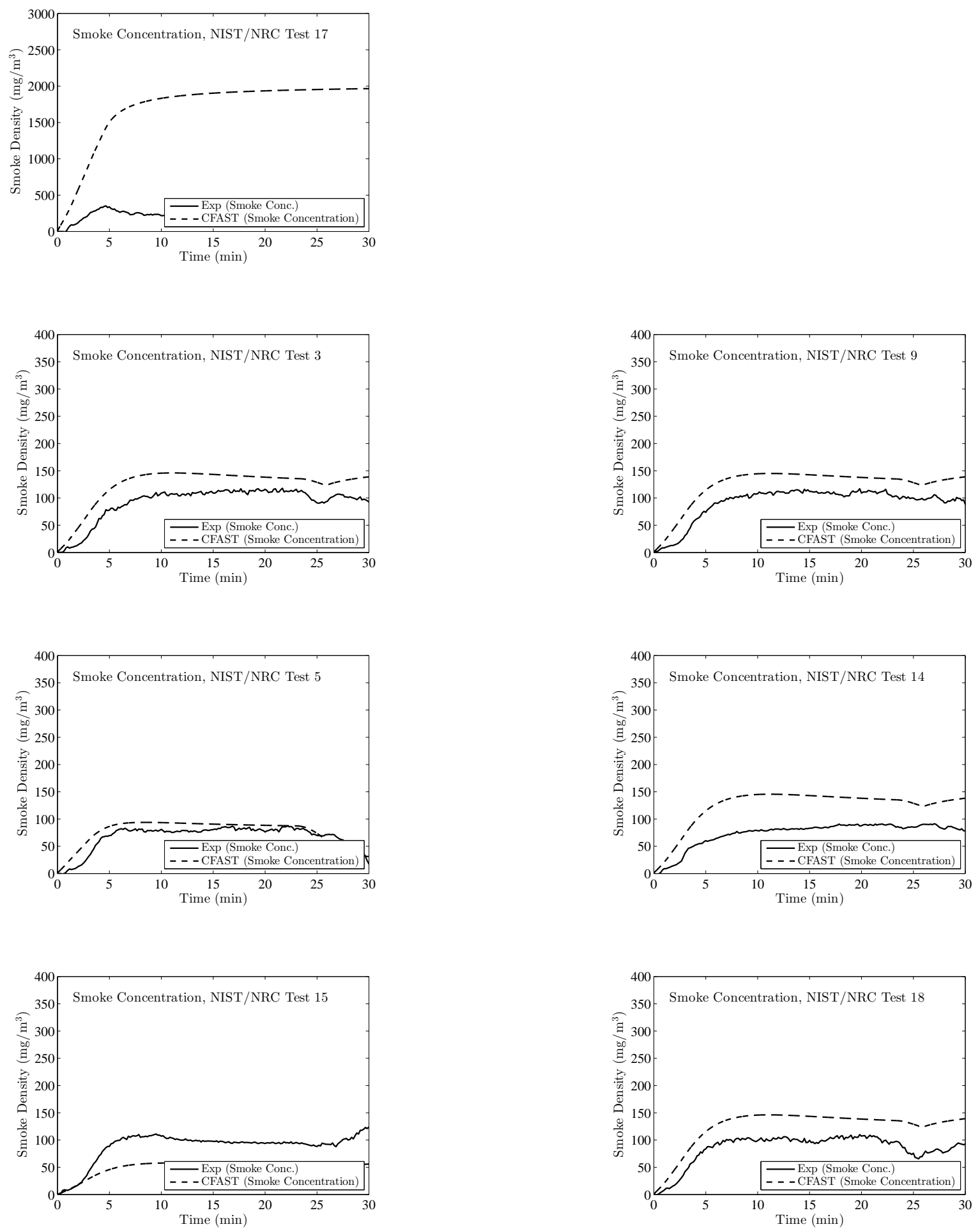

Figure B.17: Smoke Concentrationfor the NIST/NRC Series, Open Door Tests. 

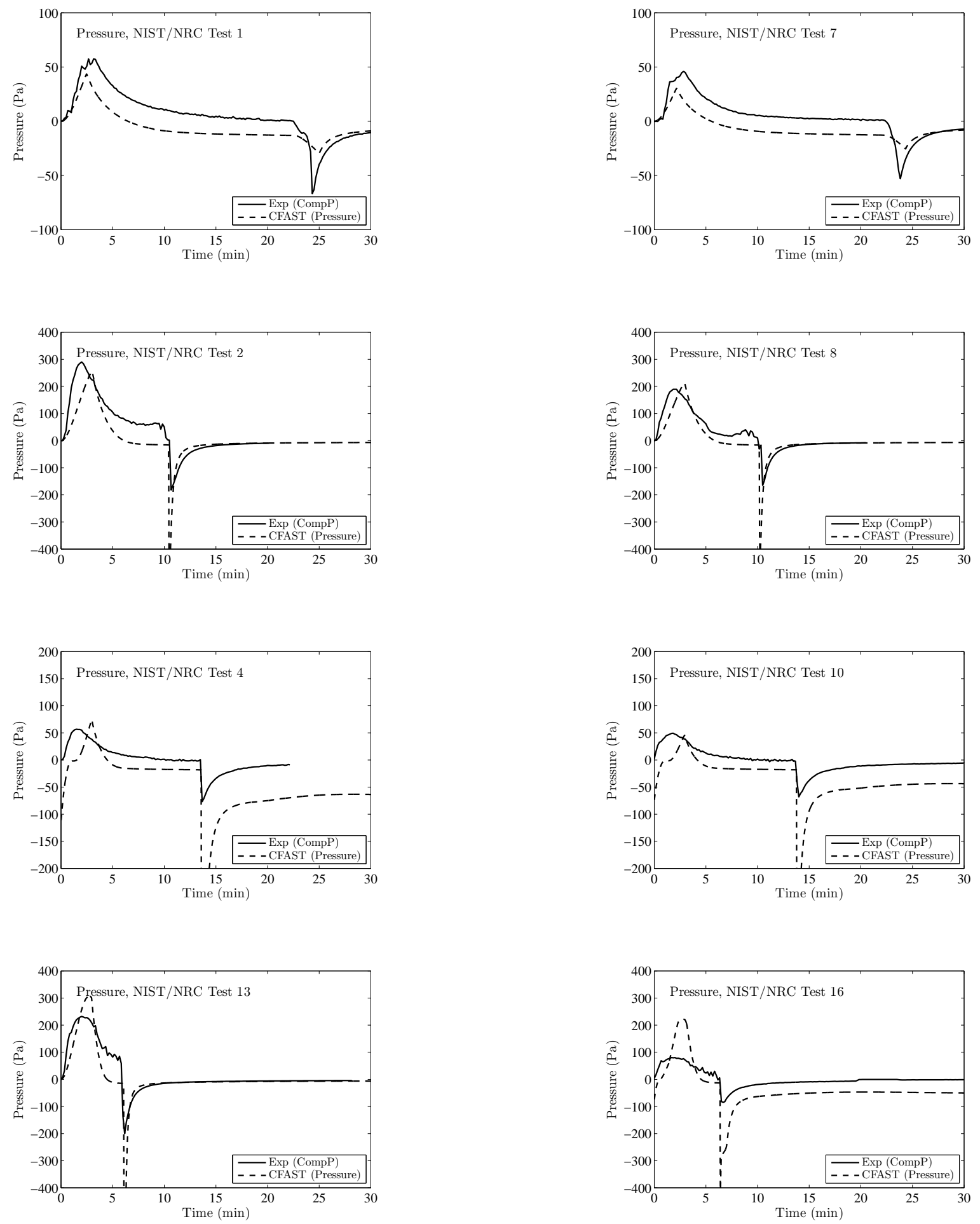

Figure B.18: Compartment Pressures for the NIST/NRC Series, Closed Door Tests. 

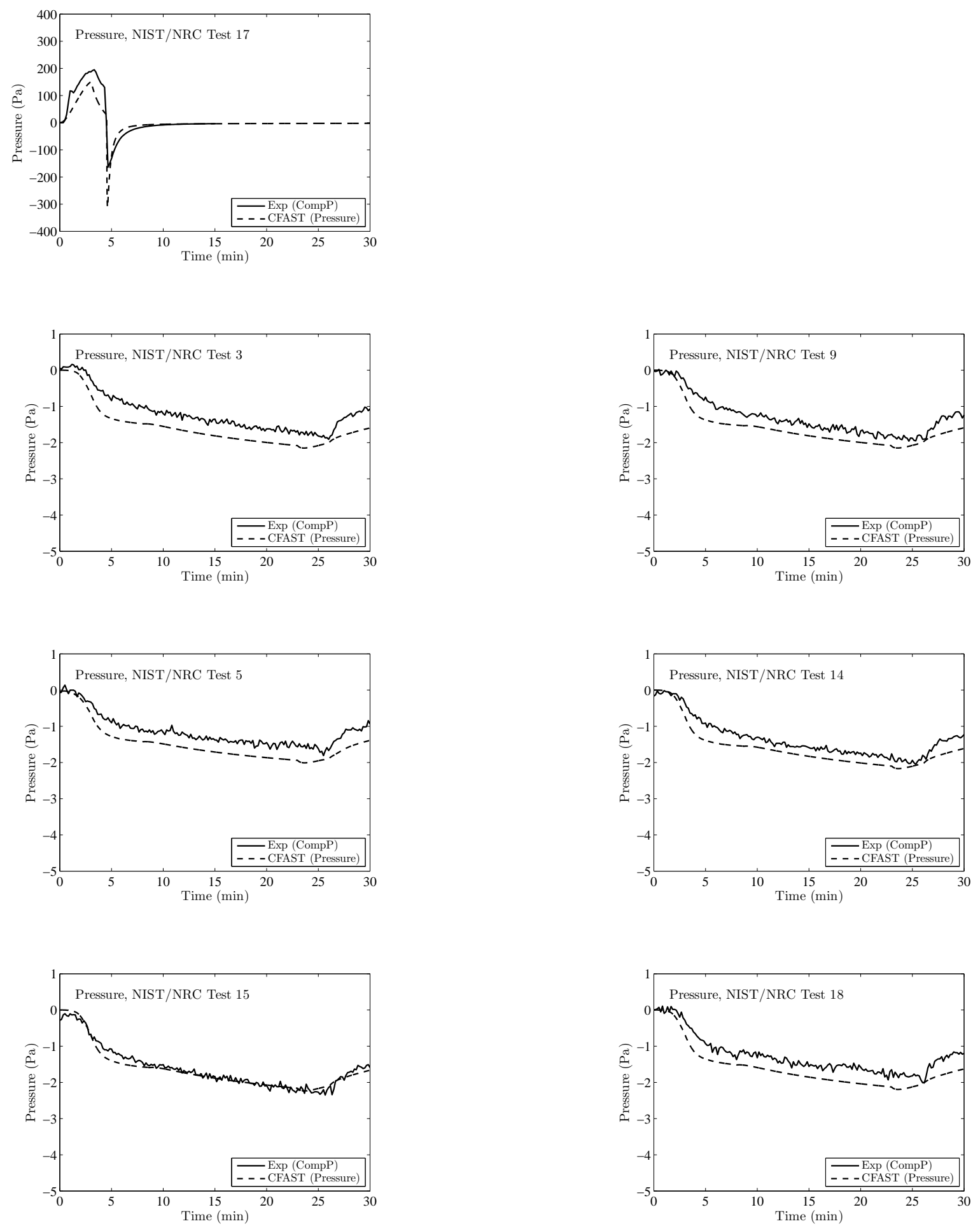

Figure B.19: Compartment Pressures for the NIST/NRC Series, Open Door Tests. 

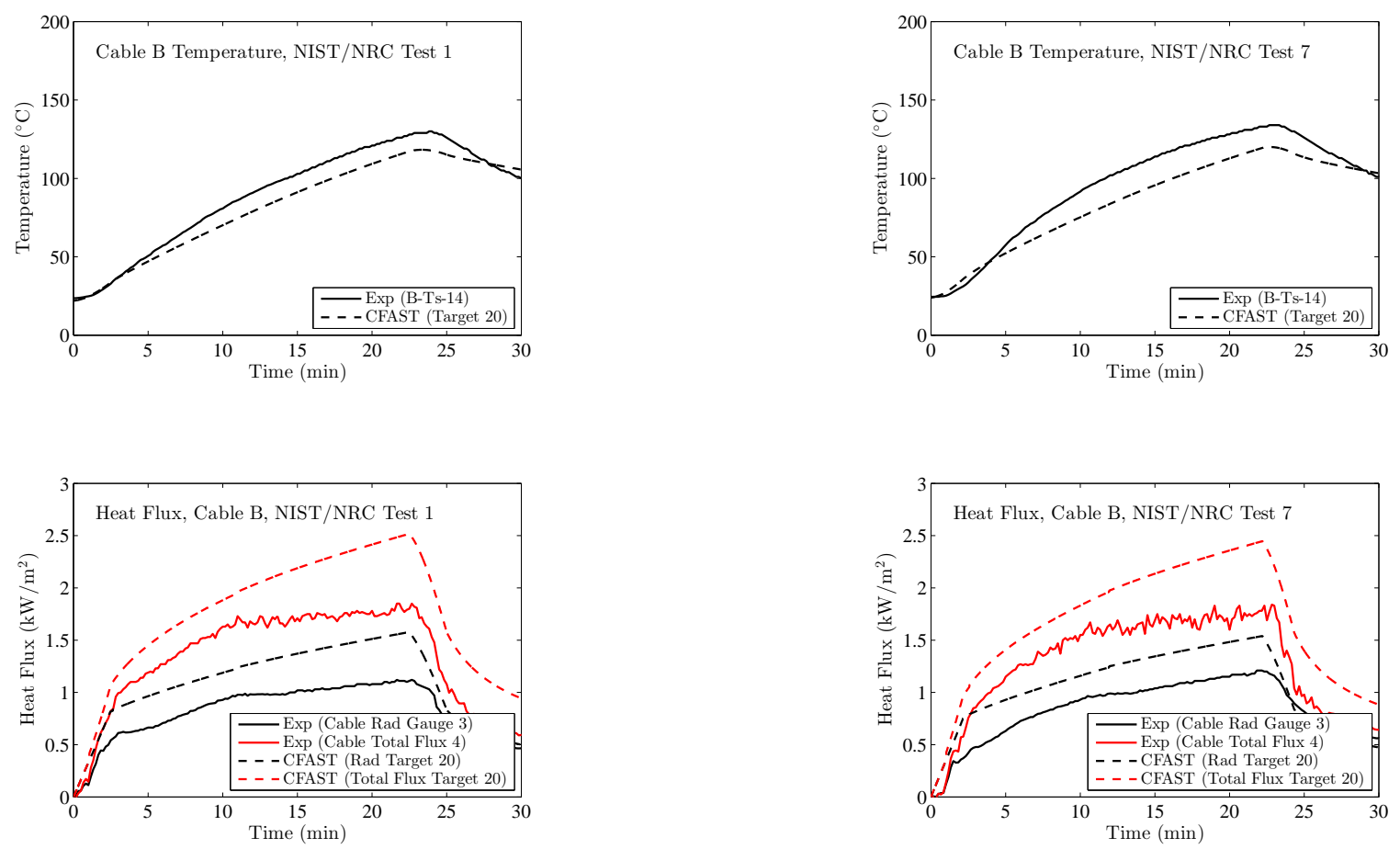

Figure B.20: NIST/NRC Series, Cable B Temperature and Heat Flux, Replicate Tests 1 and 7.
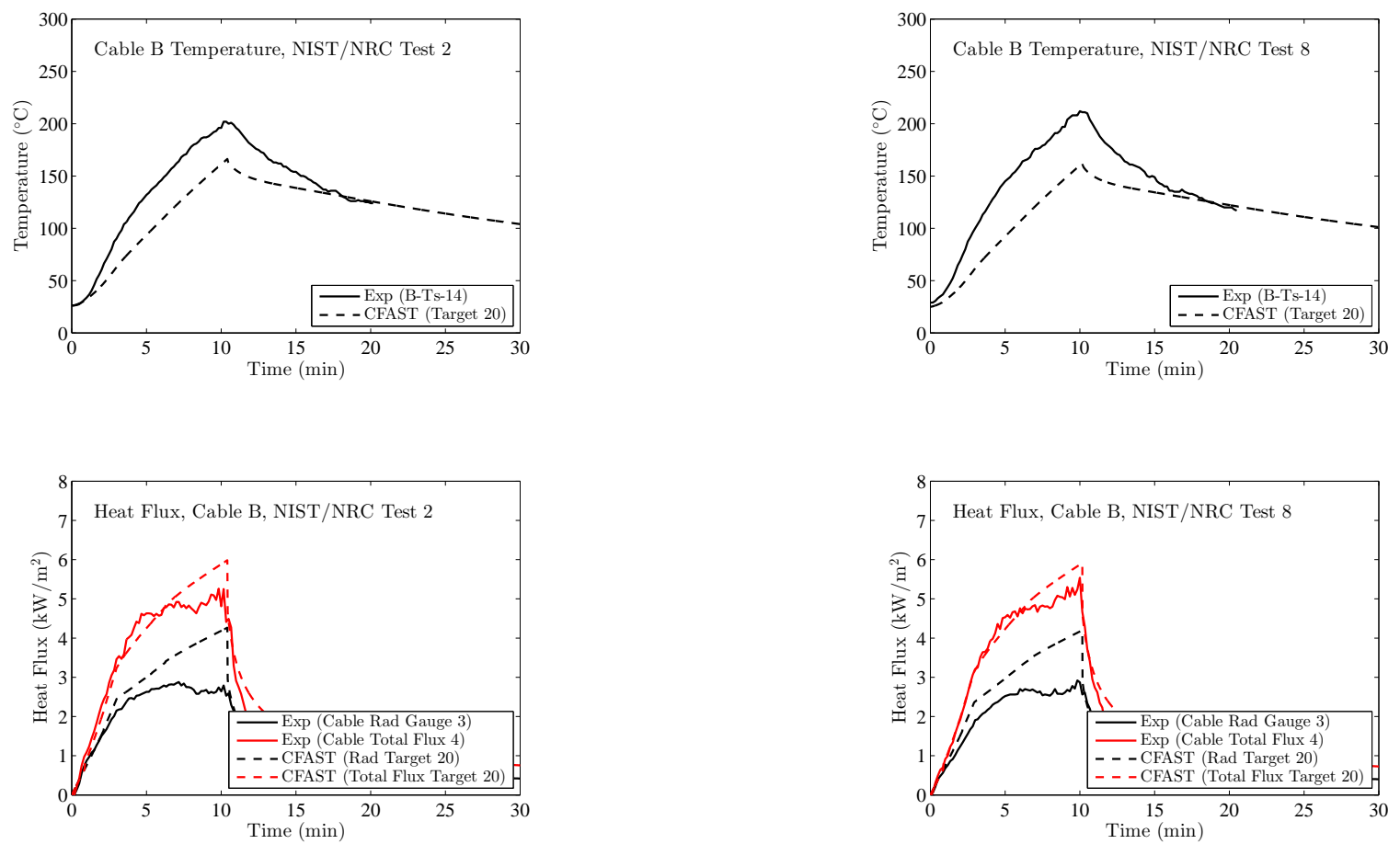

Figure B.21: NIST/NRC Series, Cable B Temperature and Heat Flux, Replicate Tests 2 and 8. 

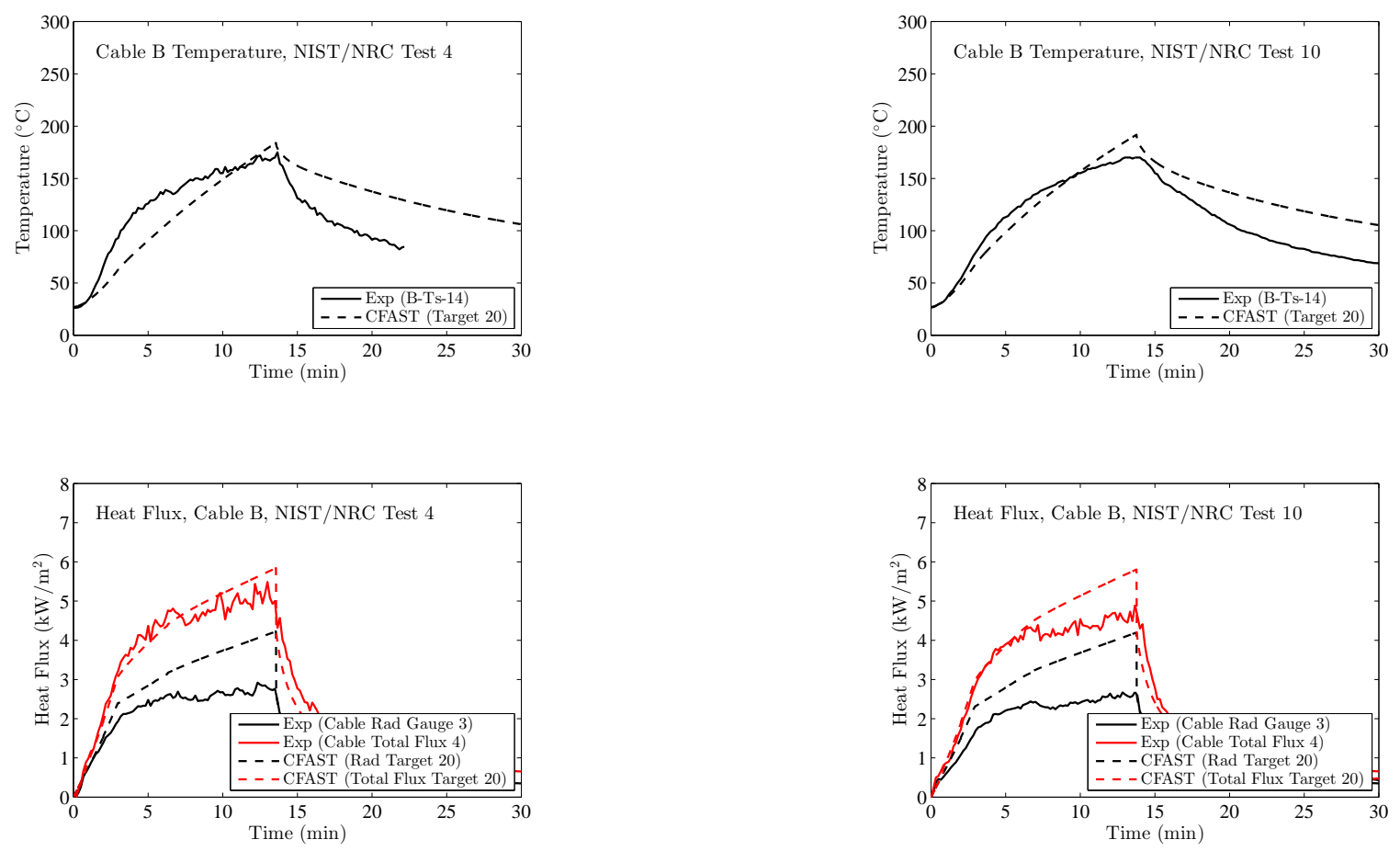

Figure B.22: NIST/NRC Series, Cable B Temperature and Heat Flux, Replicate Tests 4 and 10.
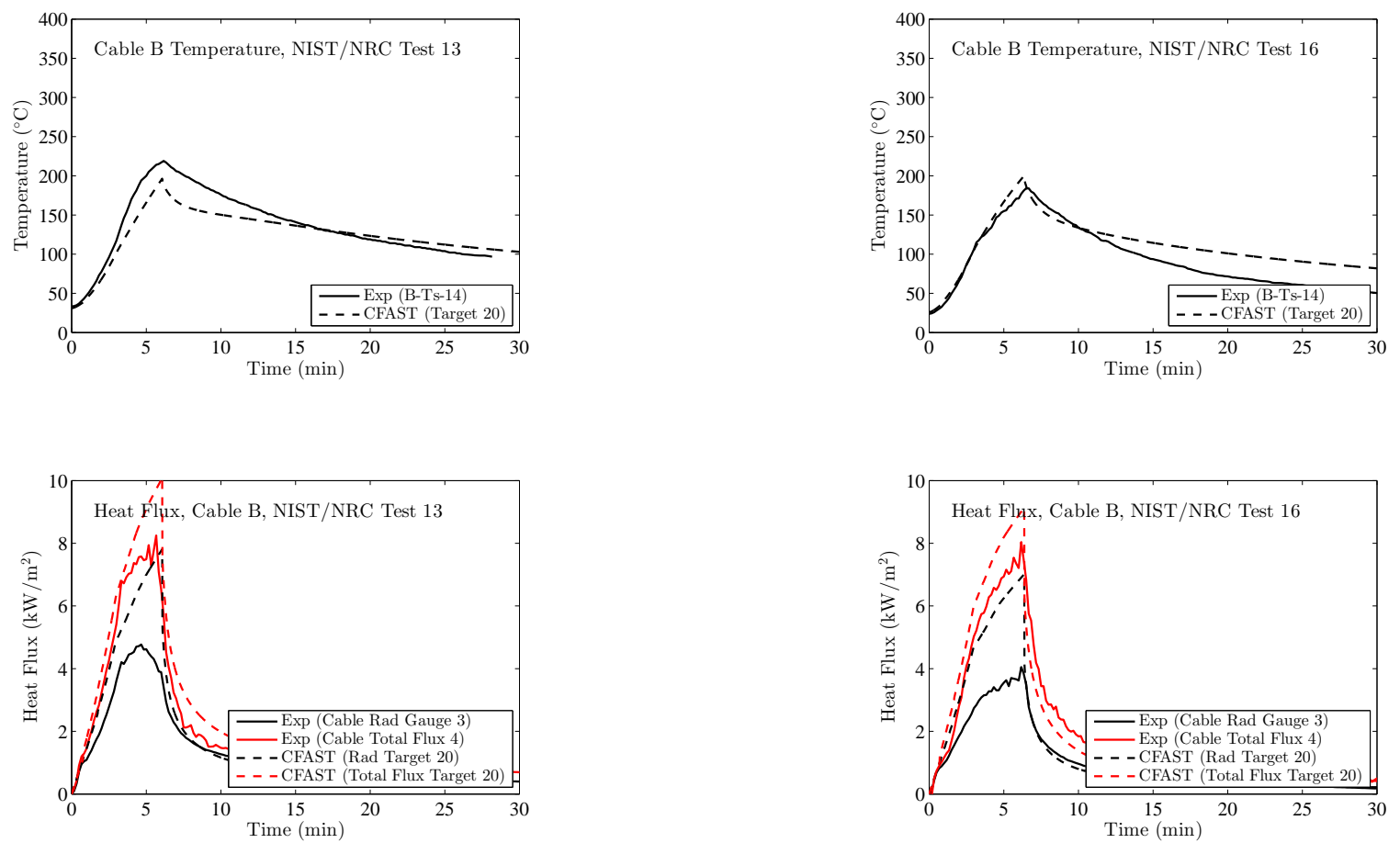

Figure B.23: NIST/NRC Series, Cable B Temperature and Heat Flux, Replicate Tests 13 and 16. 

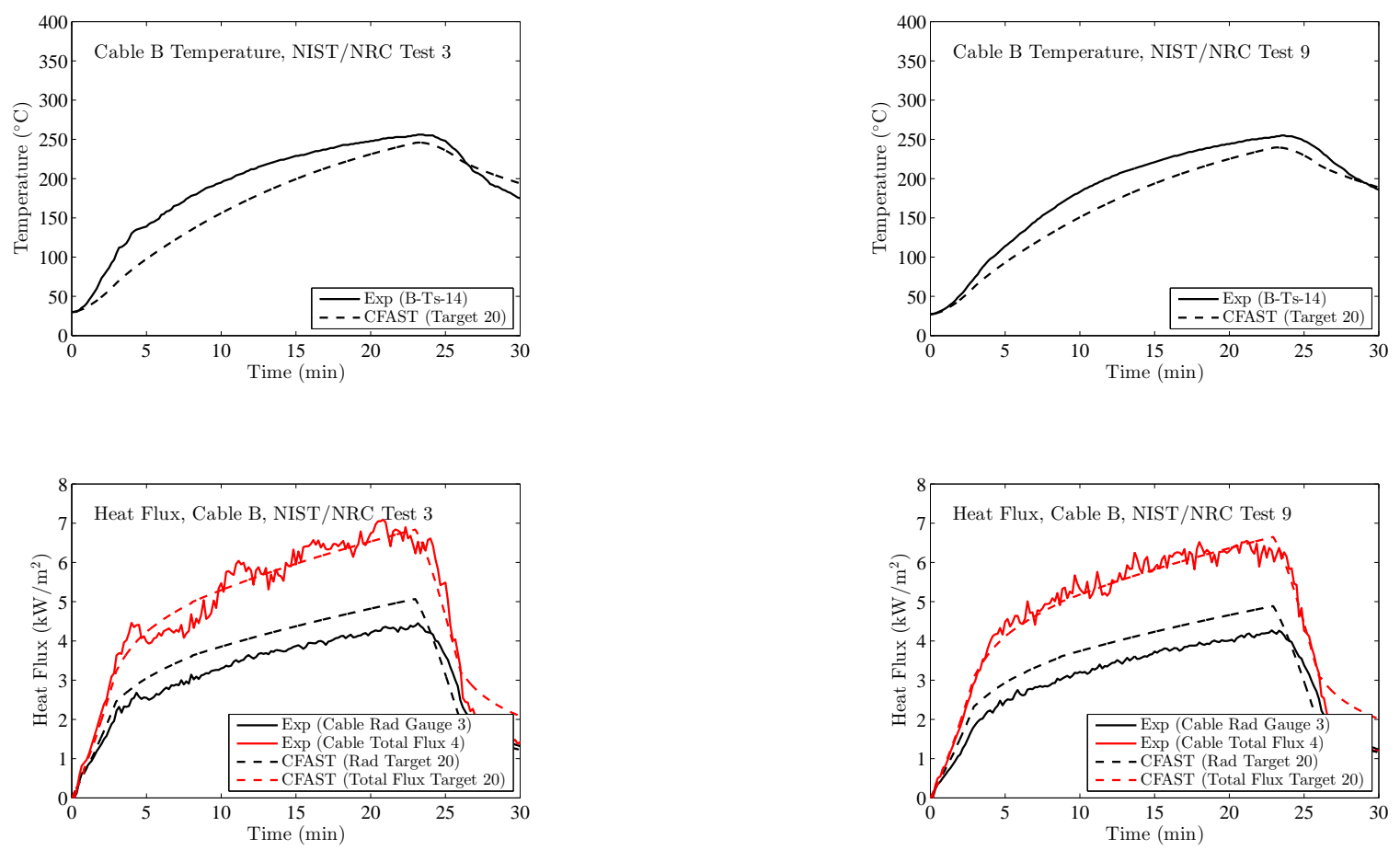

Figure B.24: NIST/NRC Series, Cable B Temperature and Heat Flux, Replicate Tests 3 and 9.
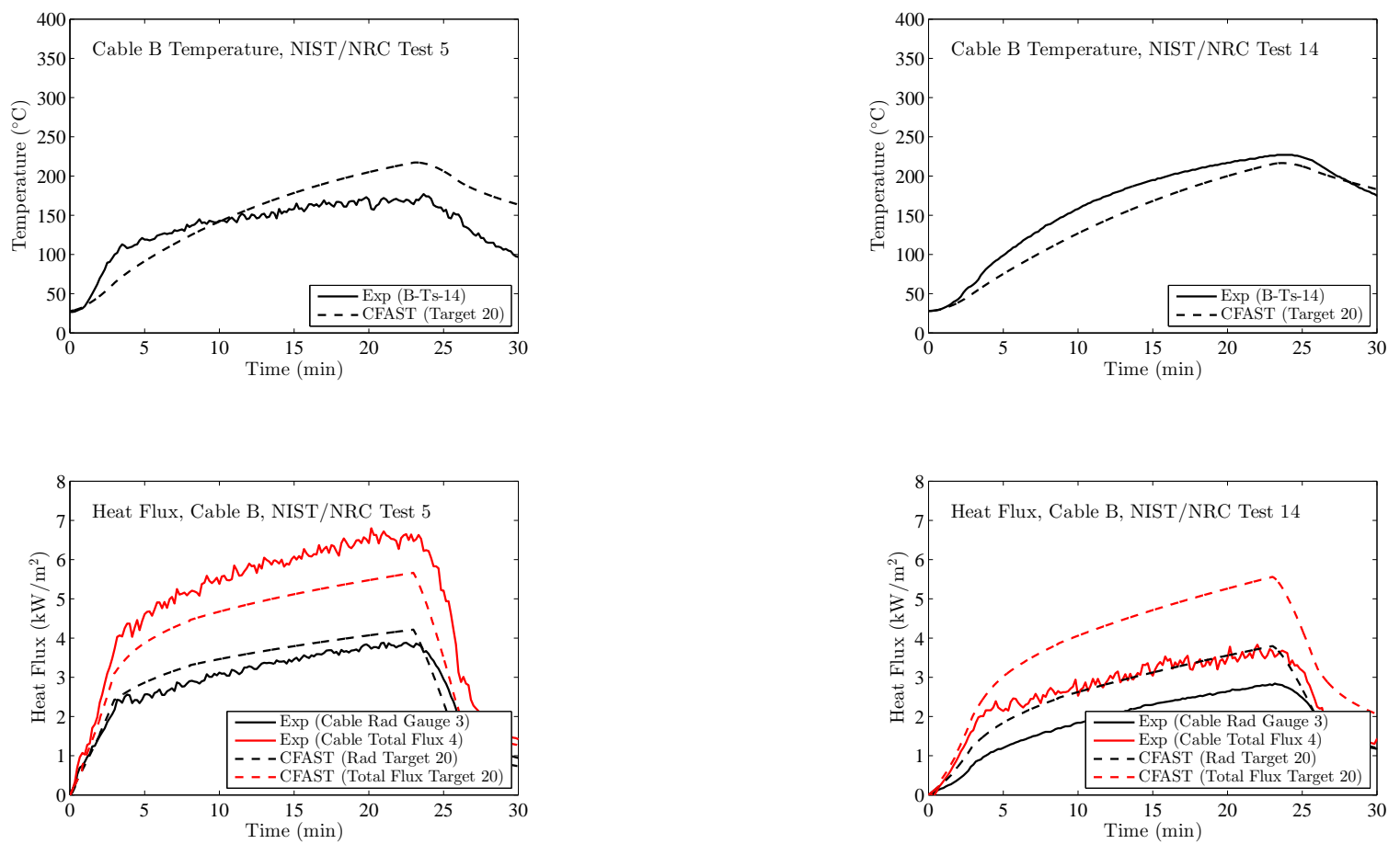

Figure B.25: NIST/NRC Series, Cable B Temperature and Heat Flux, Replicate Tests 5 and 14. 

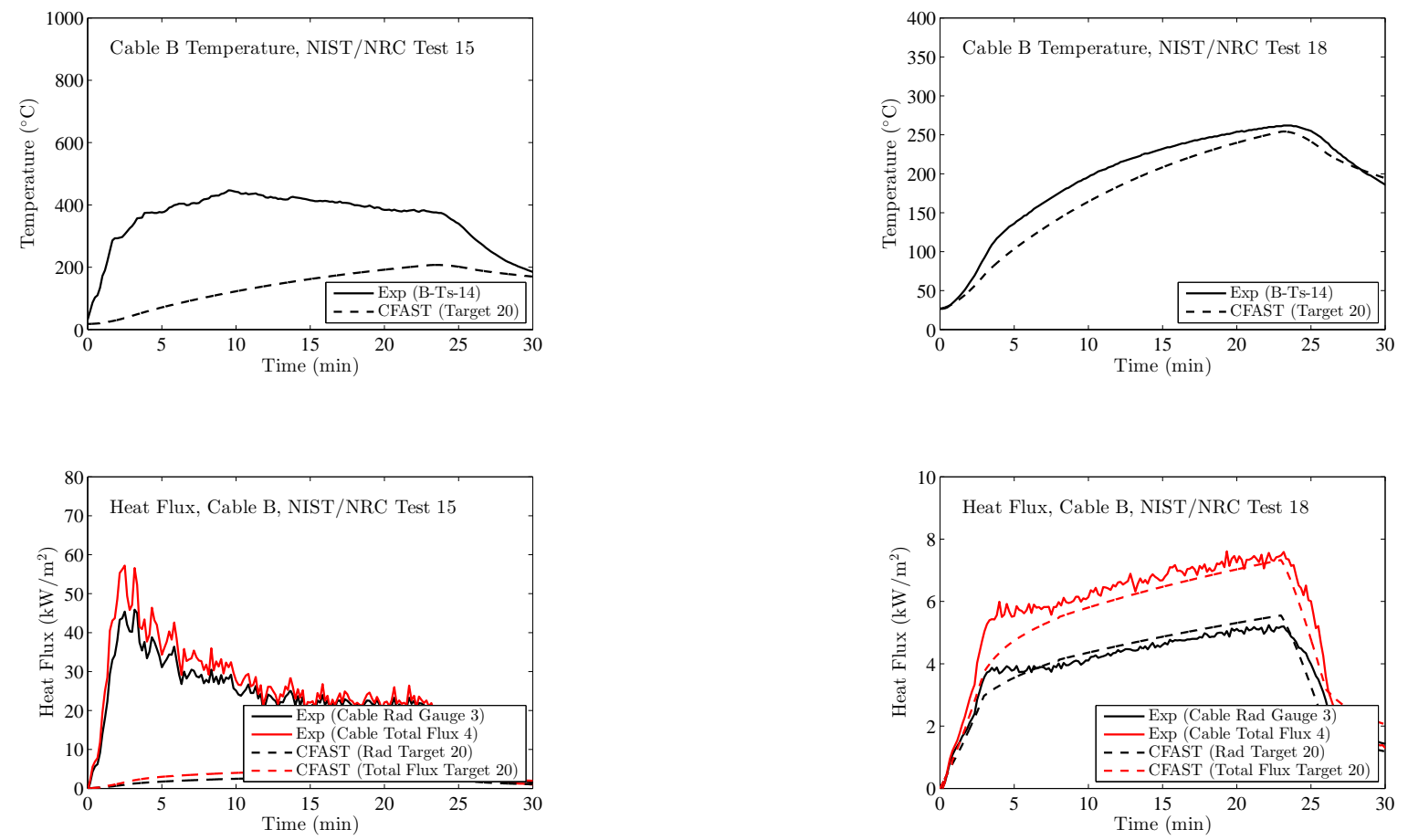

Figure B.26: NIST/NRC Series, Cable B Temperature and Heat Flux, Replicate Tests 15 and 18. 

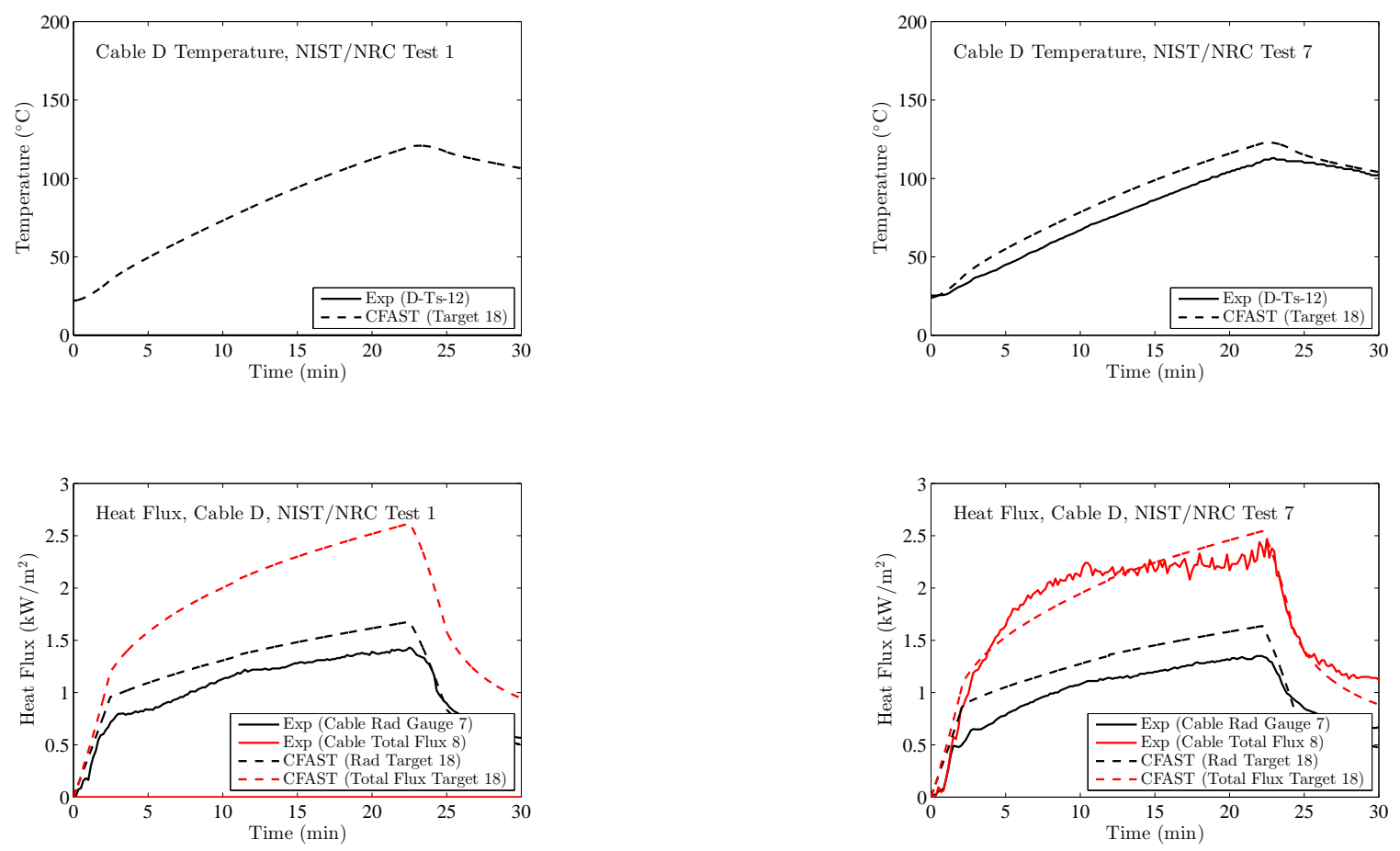

Figure B.27: NIST/NRC Series, Cable D Temperature and Heat Flux, Replicate Tests 1 and 7.
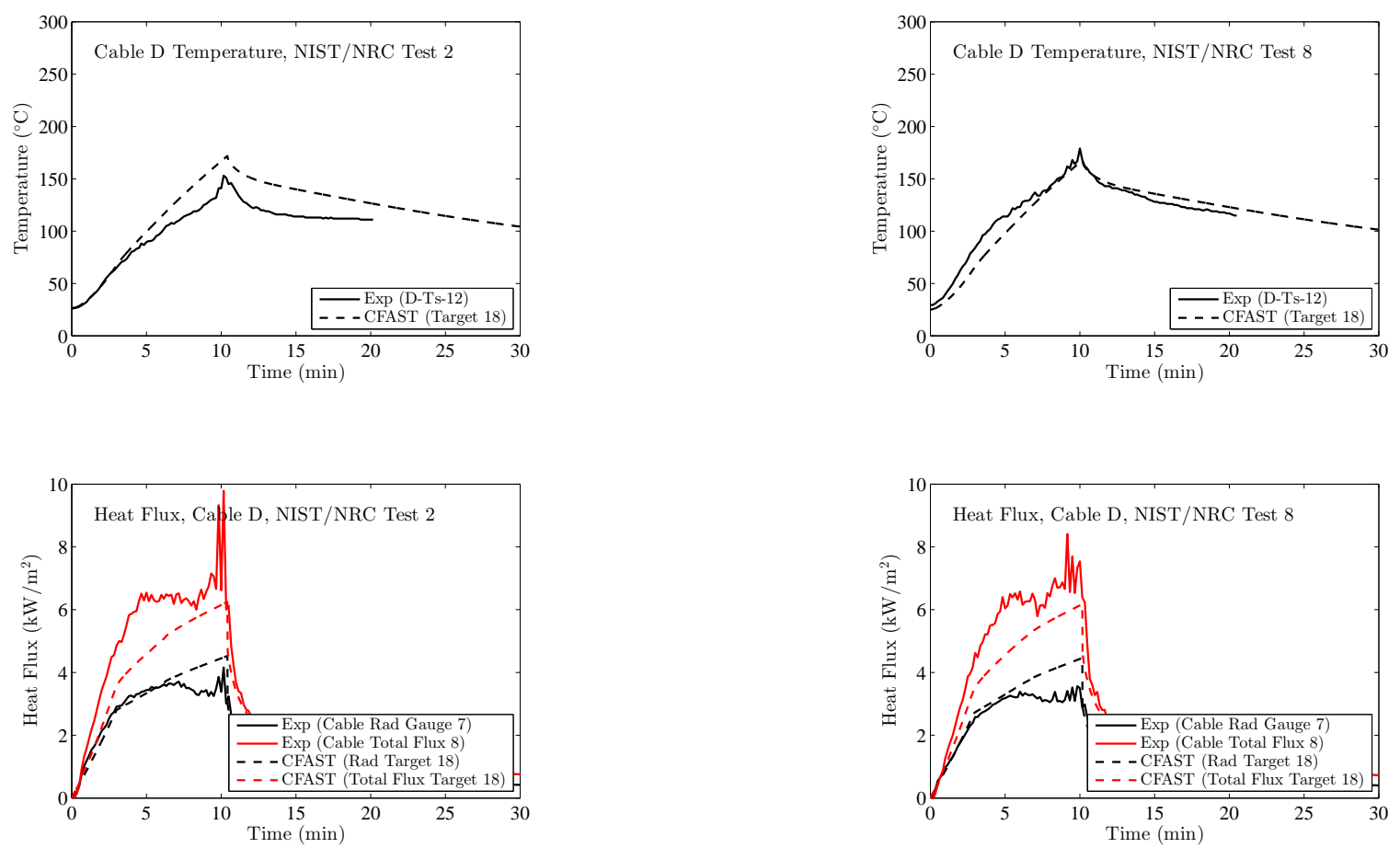

Figure B.28: NIST/NRC Series, Cable D Temperature and Heat Flux, Replicate Tests 2 and 8. 

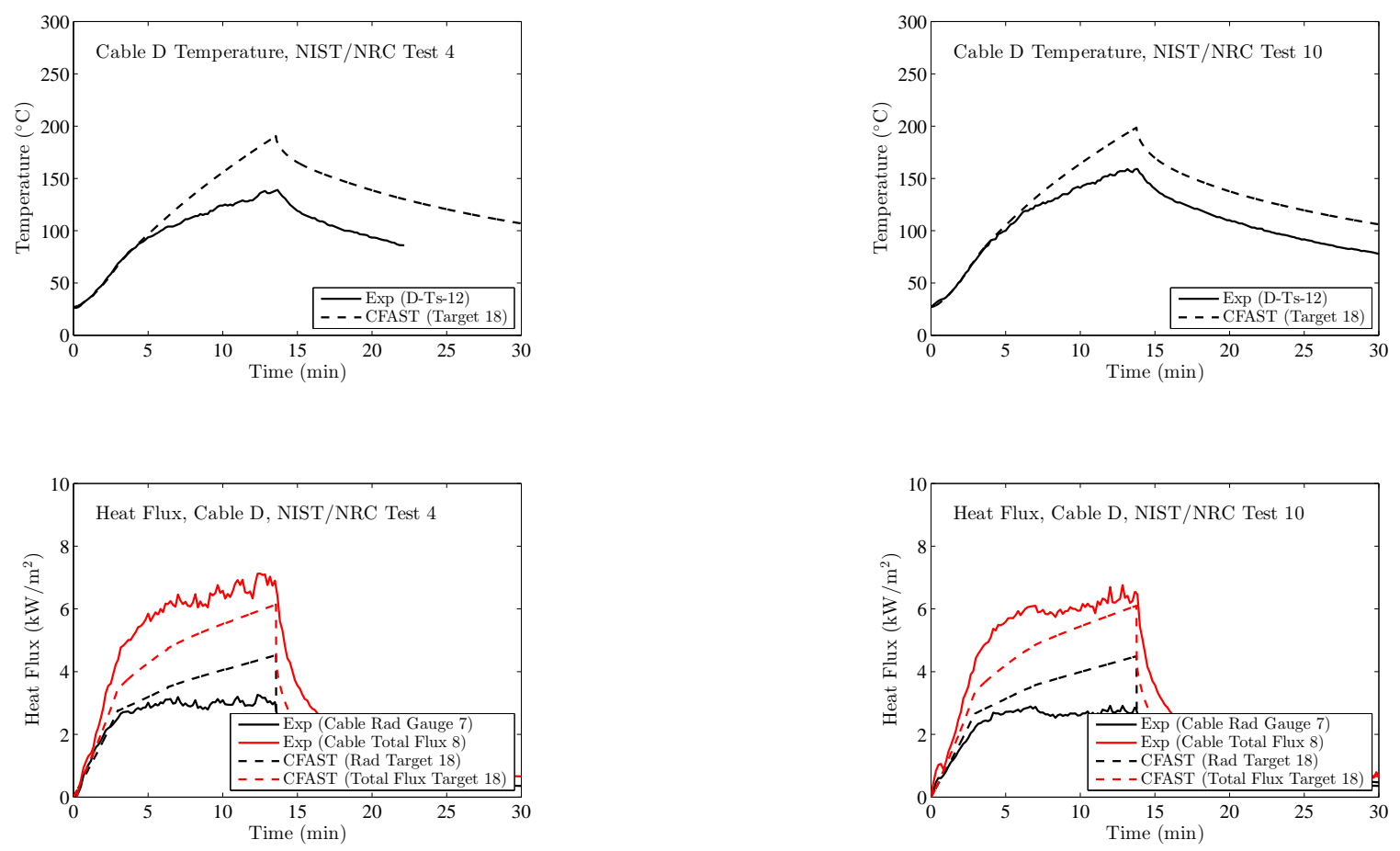

Figure B.29: NIST/NRC Series, Cable D Temperature and Heat Flux, Replicate Tests 4 and 10.
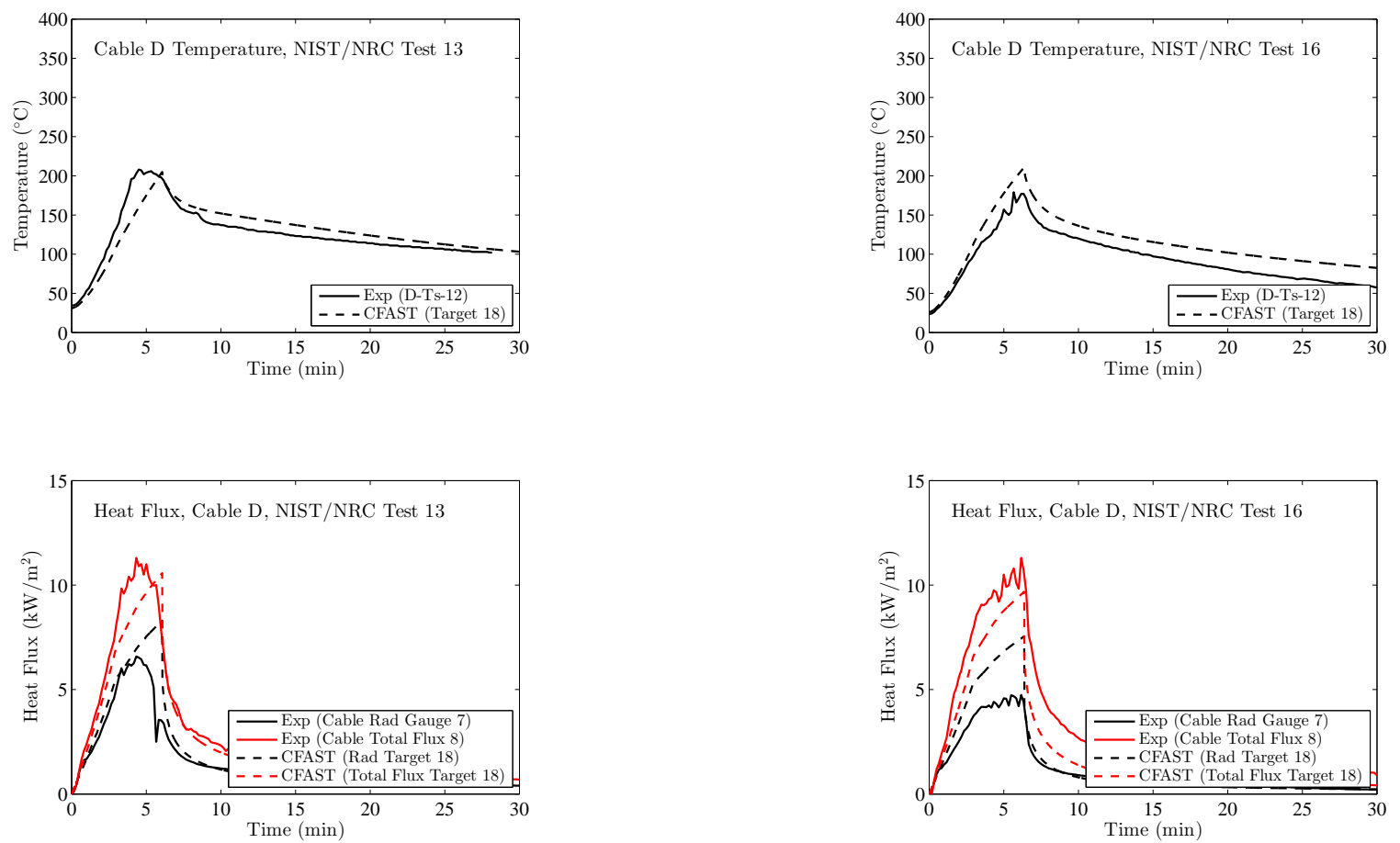

Figure B.30: NIST/NRC Series, Cable D Temperature and Heat Flux, Replicate Tests 13 and 16. 

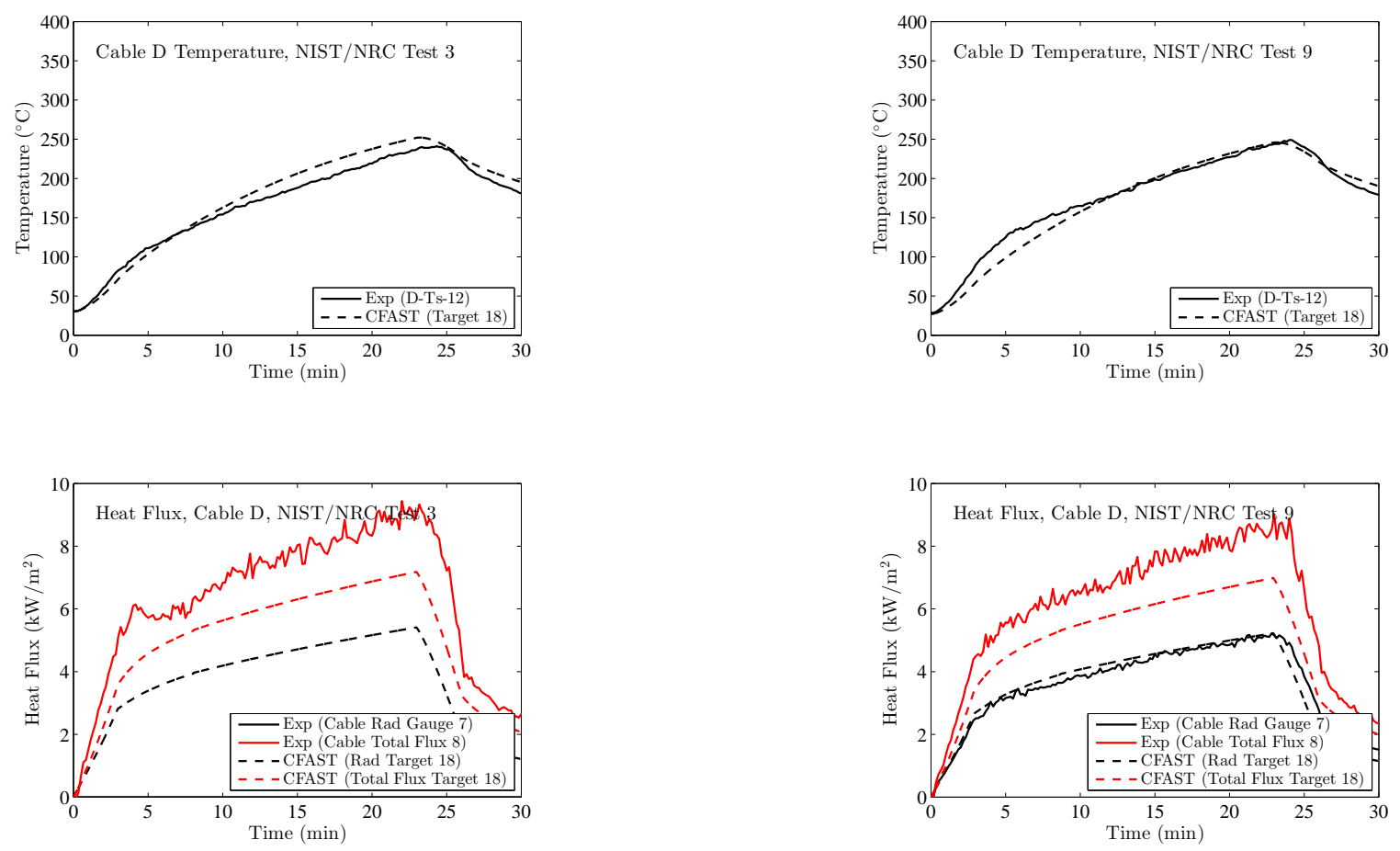

Figure B.31: NIST/NRC Series, Cable D Temperature and Heat Flux, Replicate Tests 3 and 9.
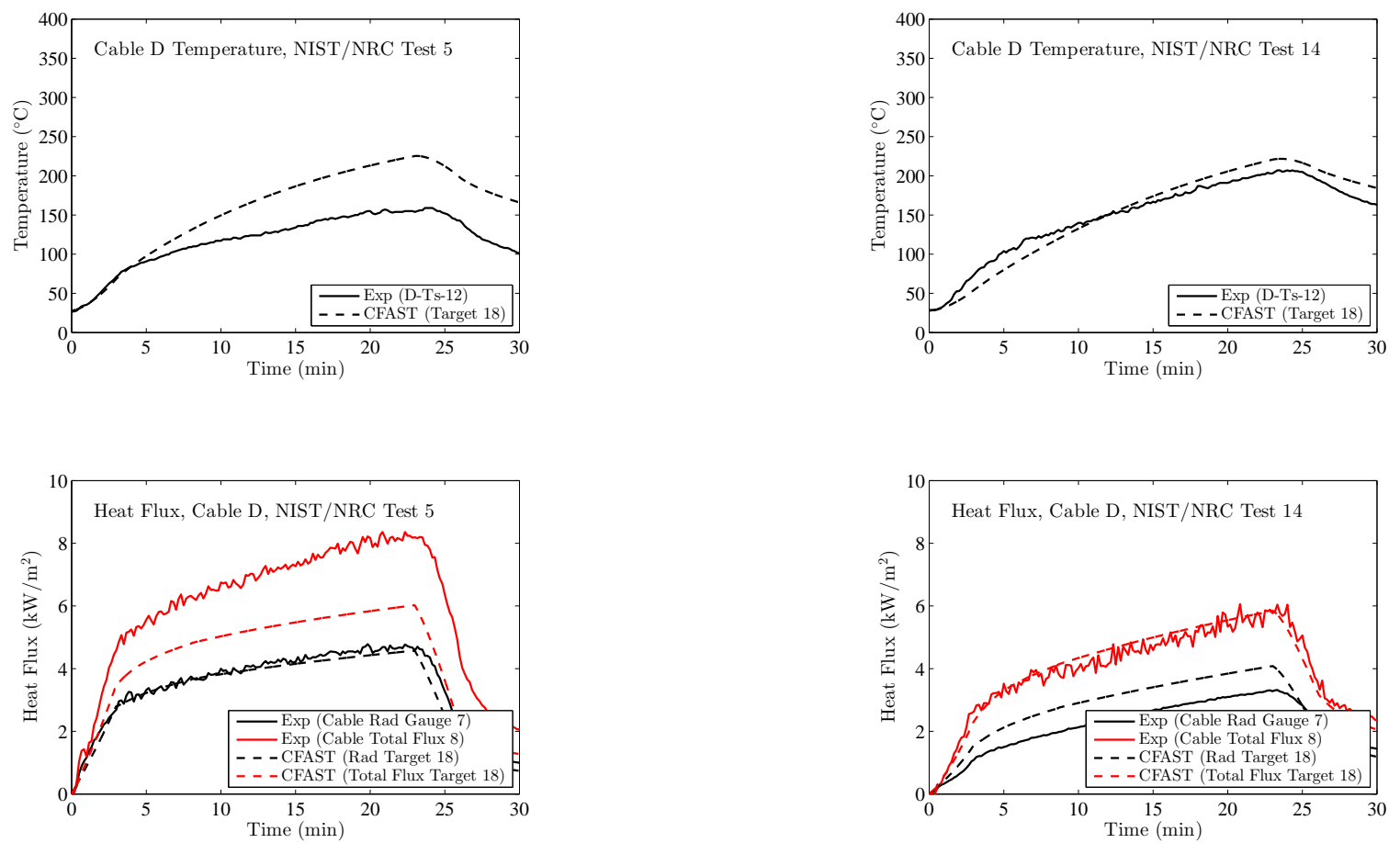

Figure B.32: NIST/NRC Series, Cable D Temperature and Heat Flux, Replicate Tests 5 and 14. 

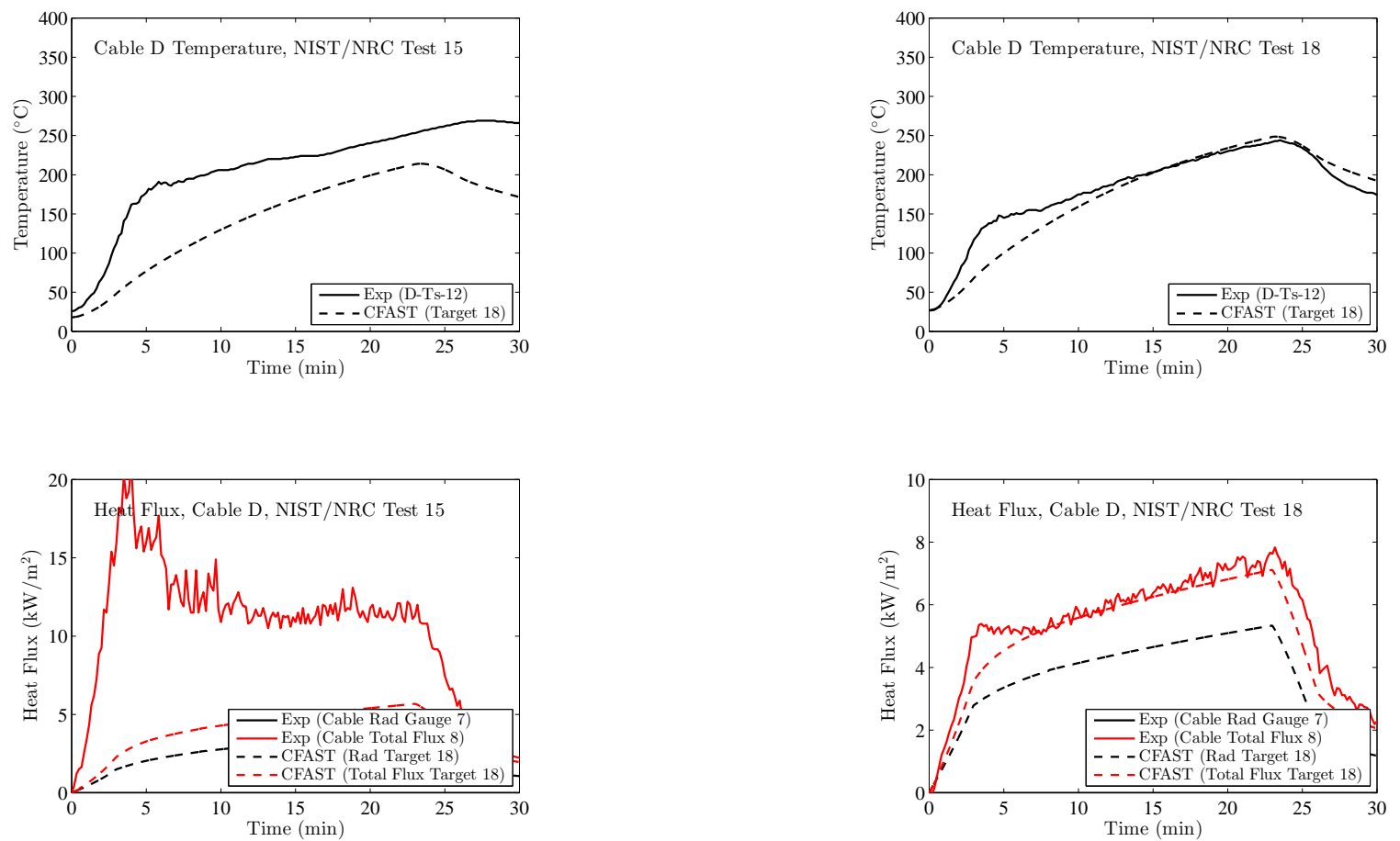

Figure B.33: NIST/NRC Series, Cable D Temperature and Heat Flux, Replicate Tests 15 and 18. 

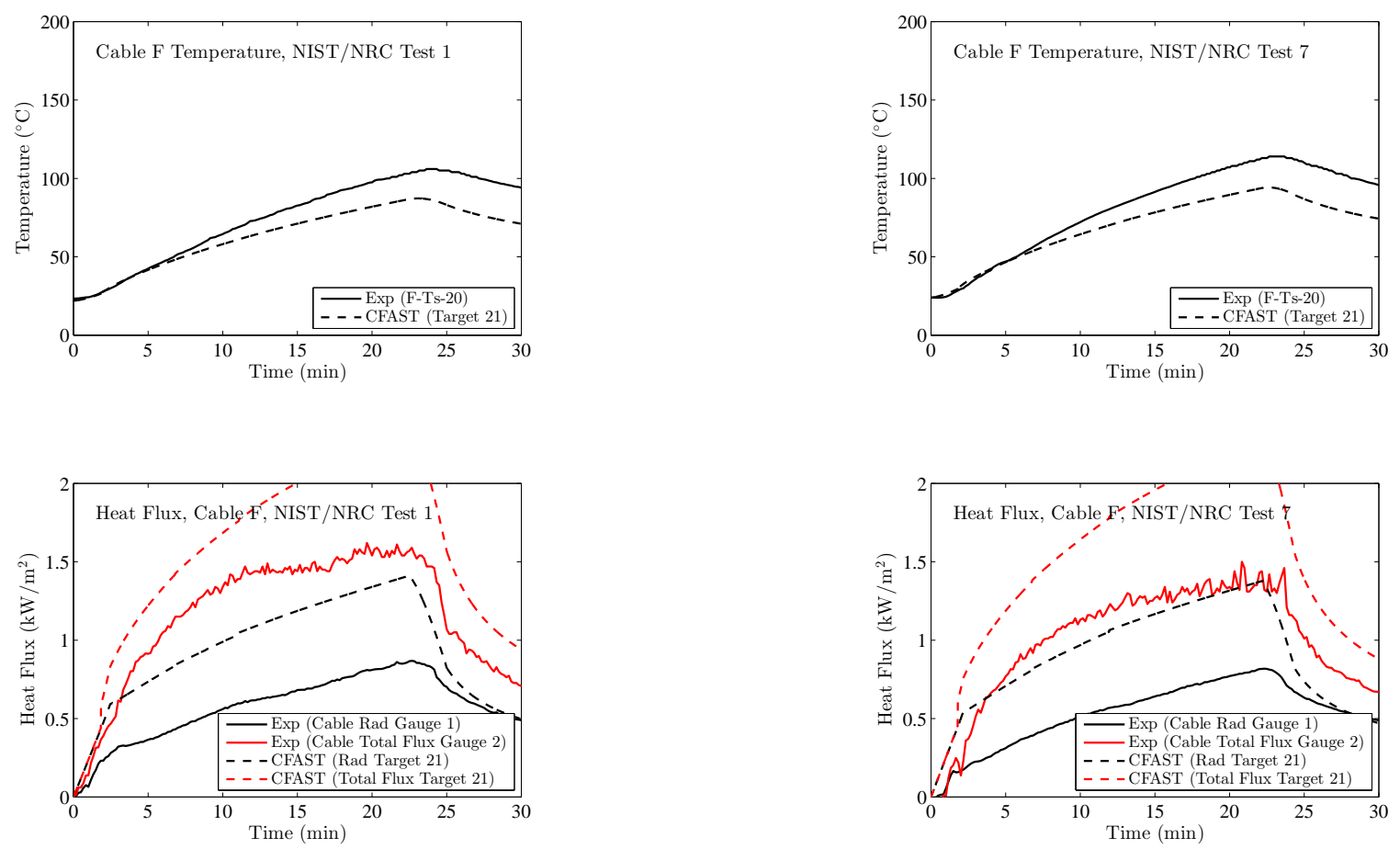

Figure B.34: NIST/NRC Series, Cable F Temperature and Heat Flux, Replicate Tests 1 and 7.
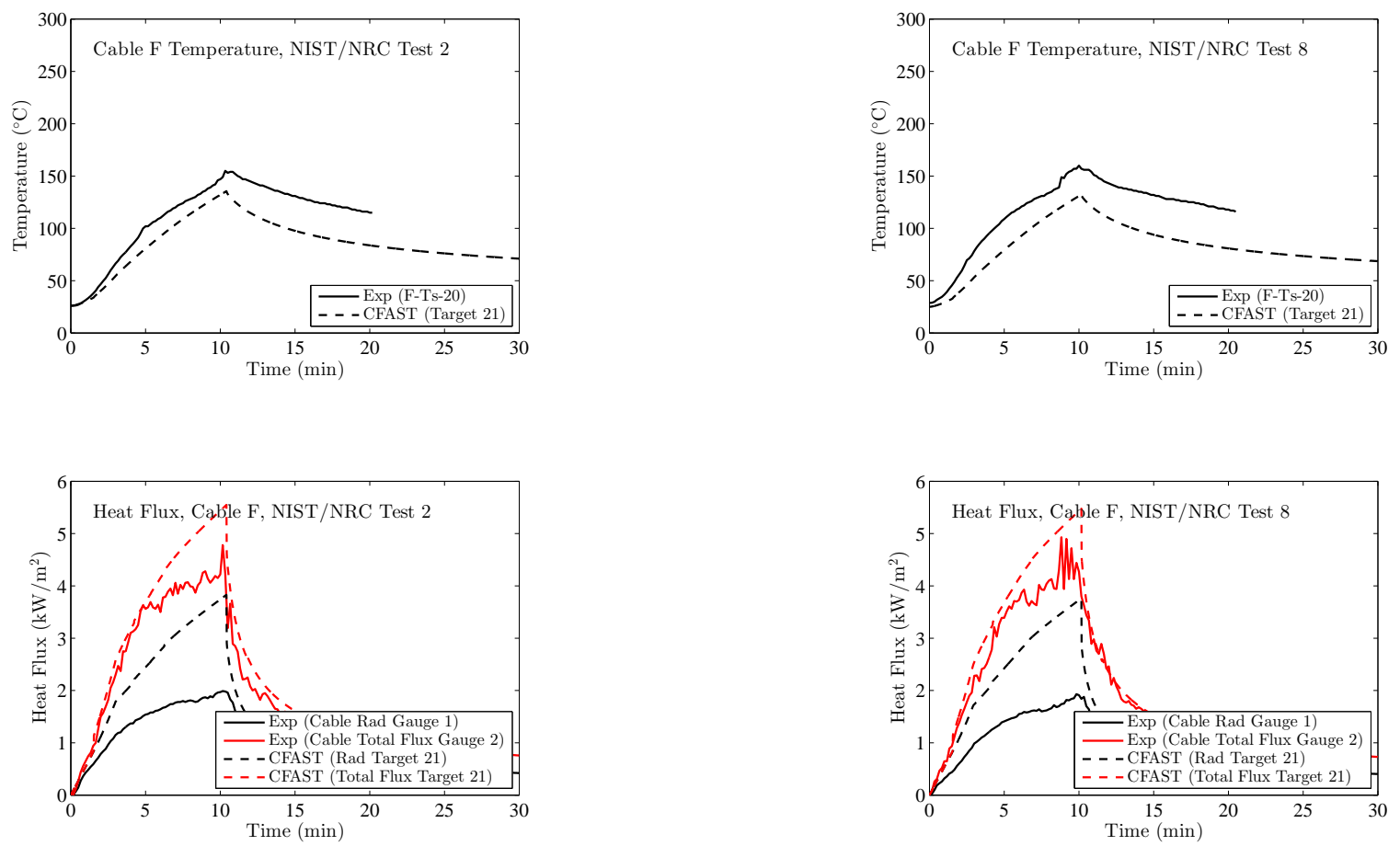

Figure B.35: NIST/NRC Series, Cable F Temperature and Heat Flux, Replicate Tests 2 and 8. 

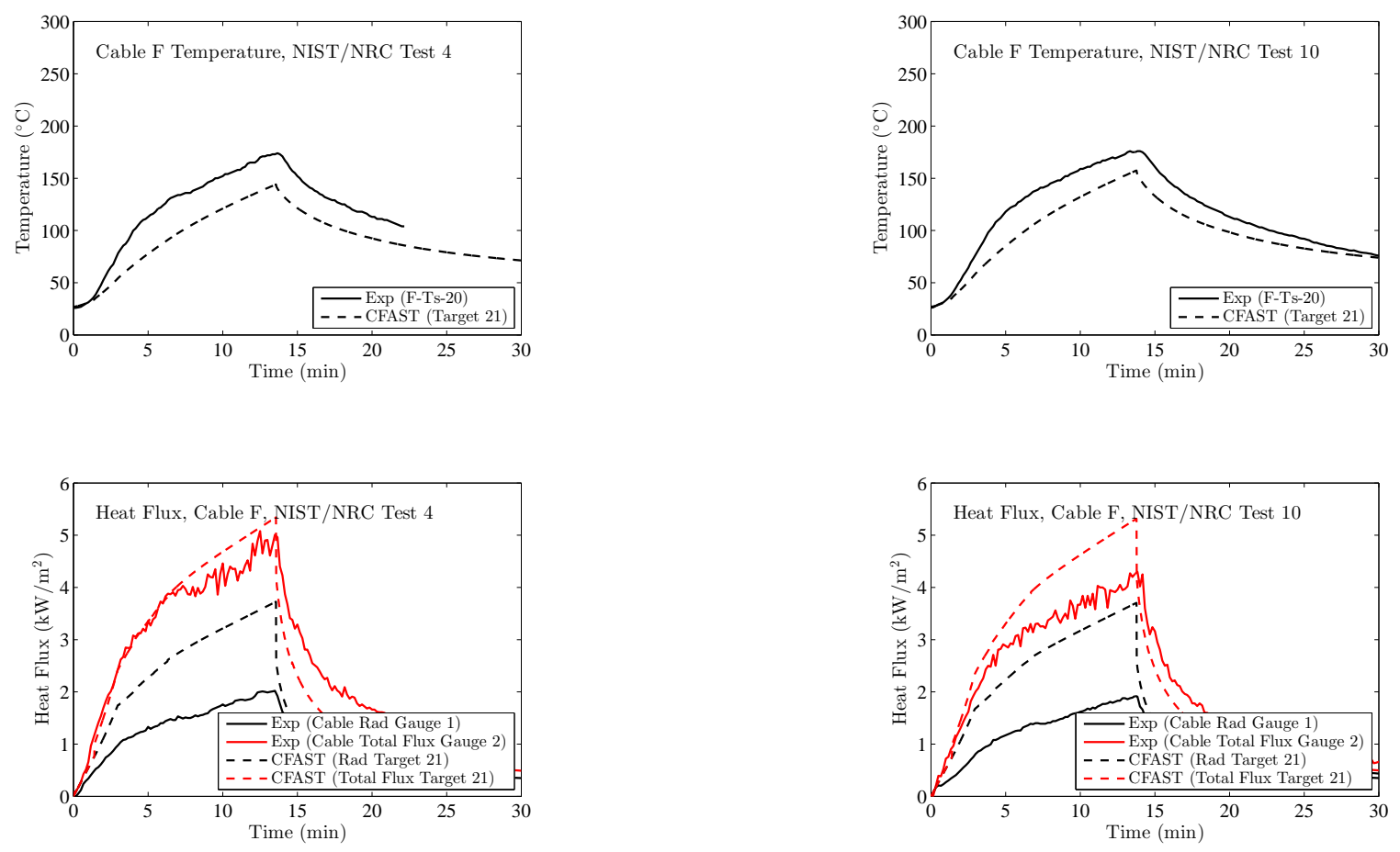

Figure B.36: NIST/NRC Series, Cable F Temperature and Heat Flux, Replicate Tests 4 and 10.
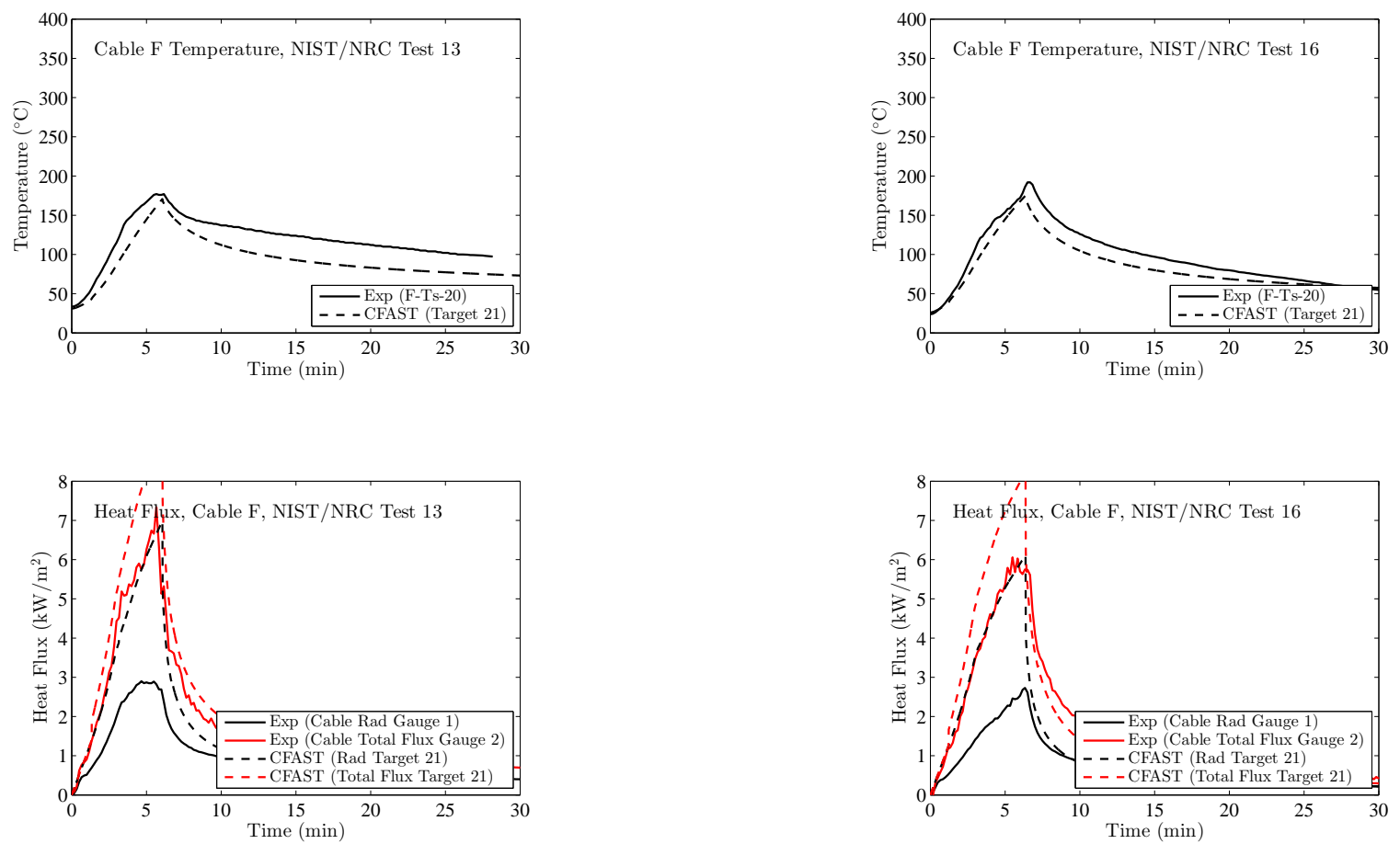

Figure B.37: NIST/NRC Series, Cable F Temperature and Heat Flux, Replicate Tests 13 and 16. 

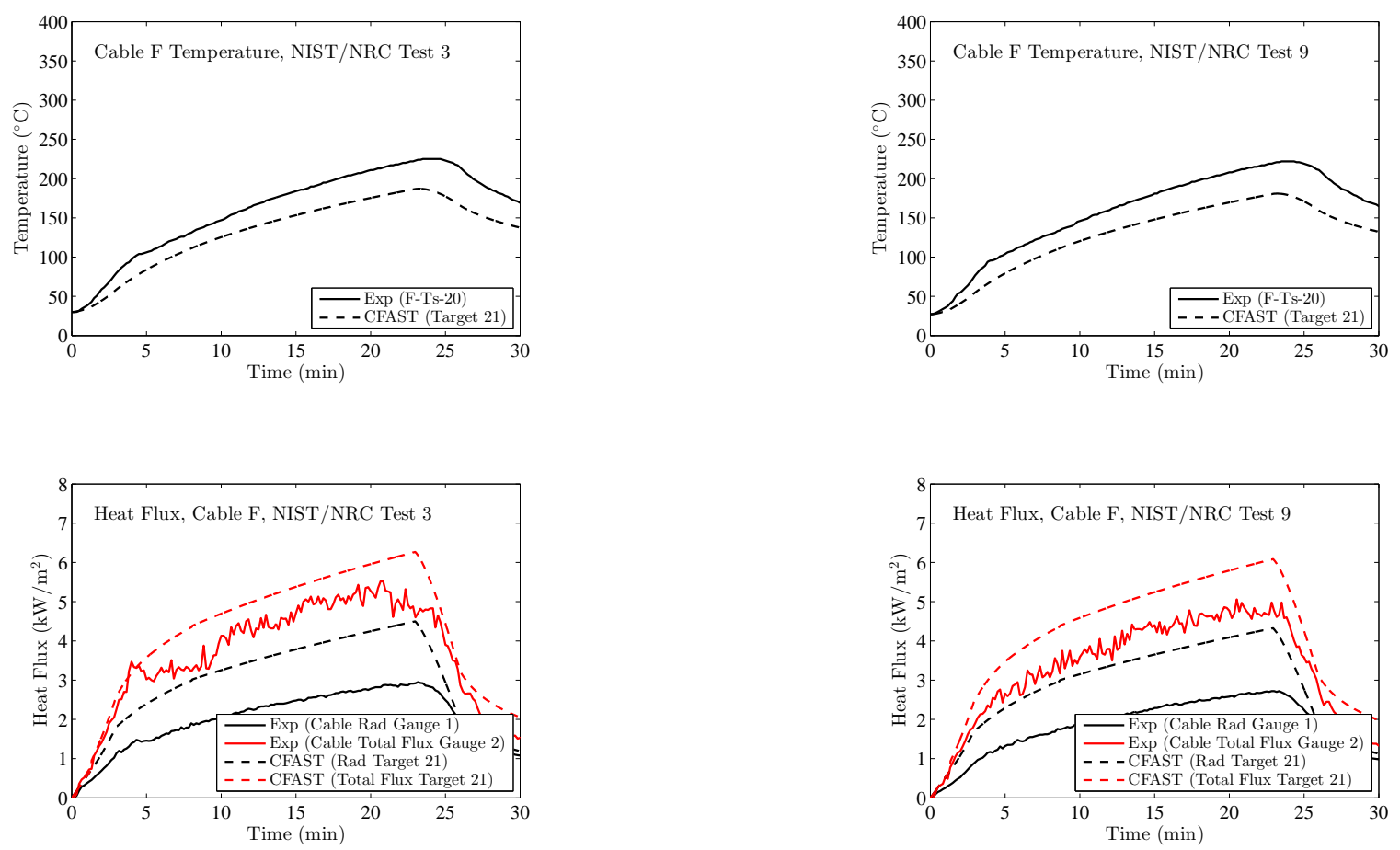

Figure B.38: NIST/NRC Series, Cable F Temperature and Heat Flux, Replicate Tests 3 and 9.
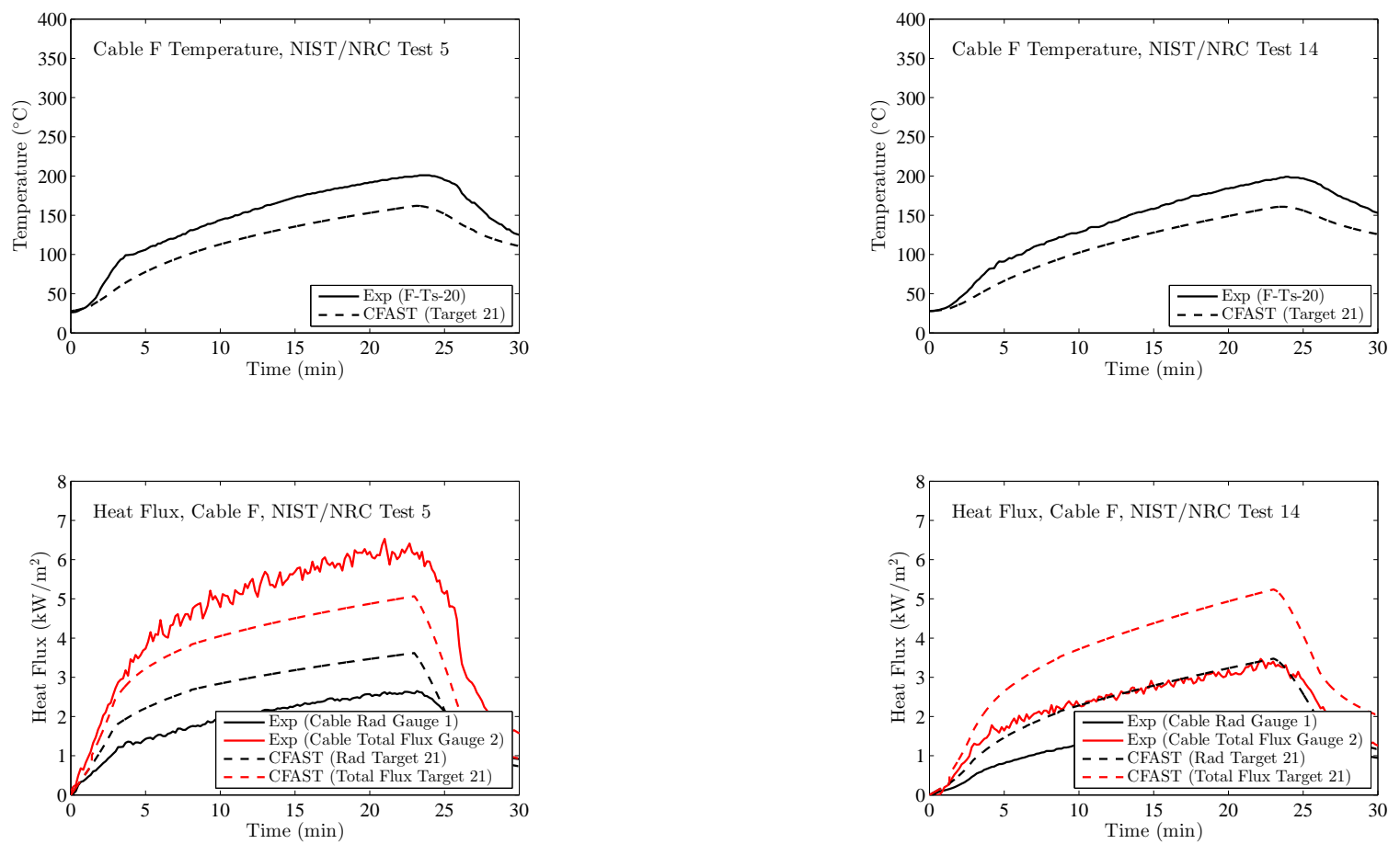

Figure B.39: NIST/NRC Series, Cable F Temperature and Heat Flux, Replicate Tests 5 and 14. 

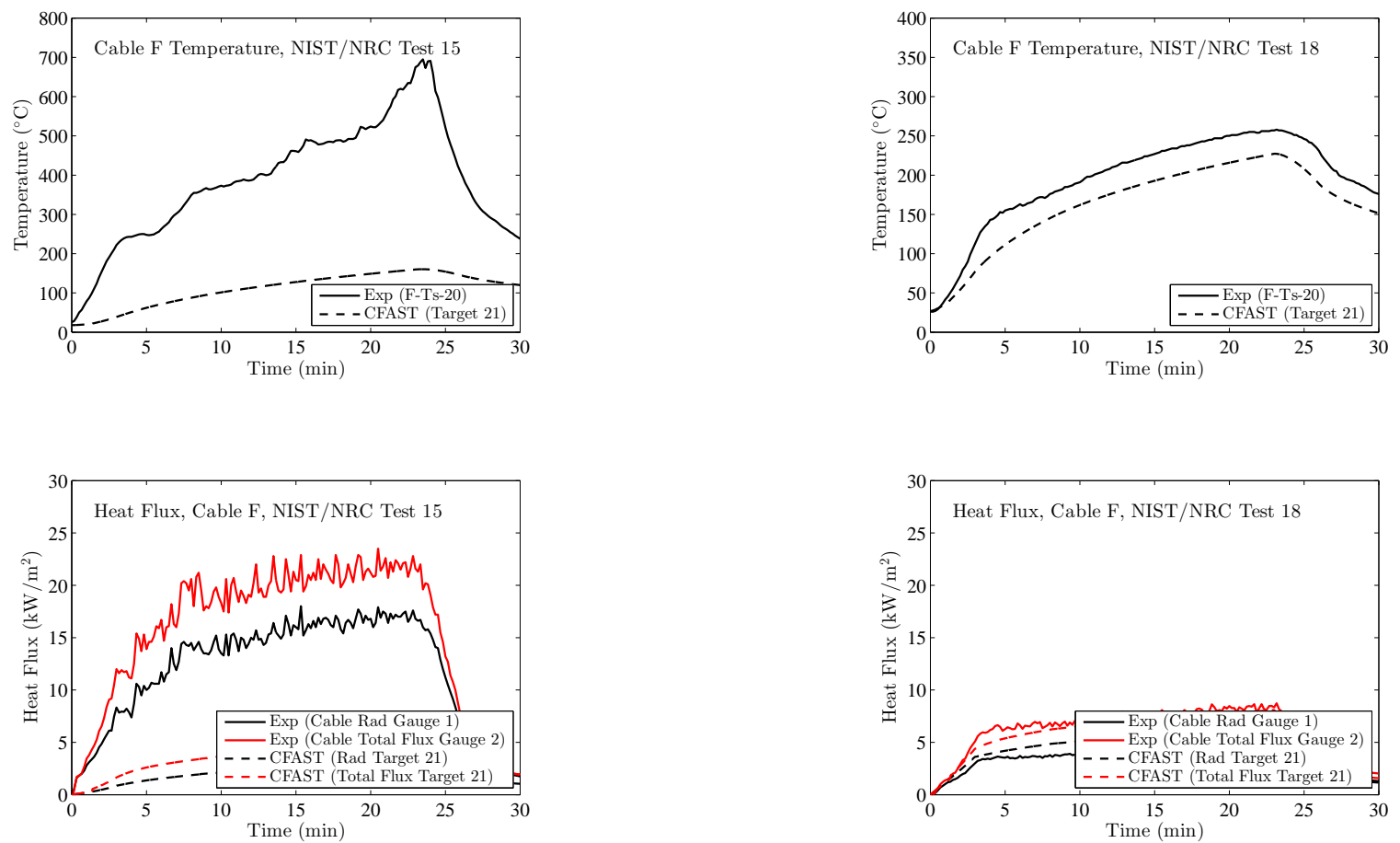

Figure B.40: NIST/NRC Series, Cable F Temperature and Heat Flux, Replicate Tests 15 and 18. 

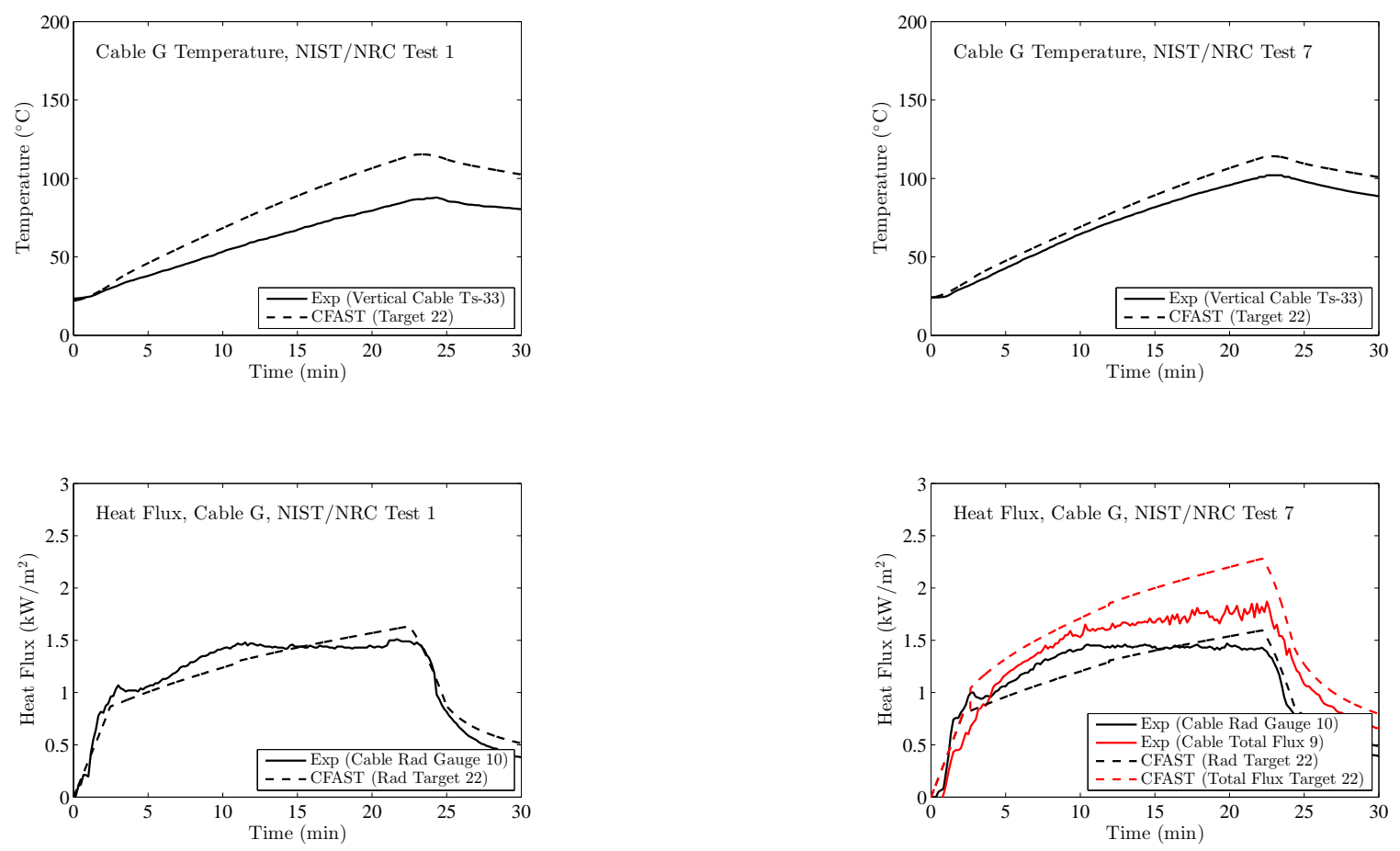

Figure B.41: NIST/NRC Series, Cable G Temperature and Heat Flux, Replicate Tests 1 and 7.
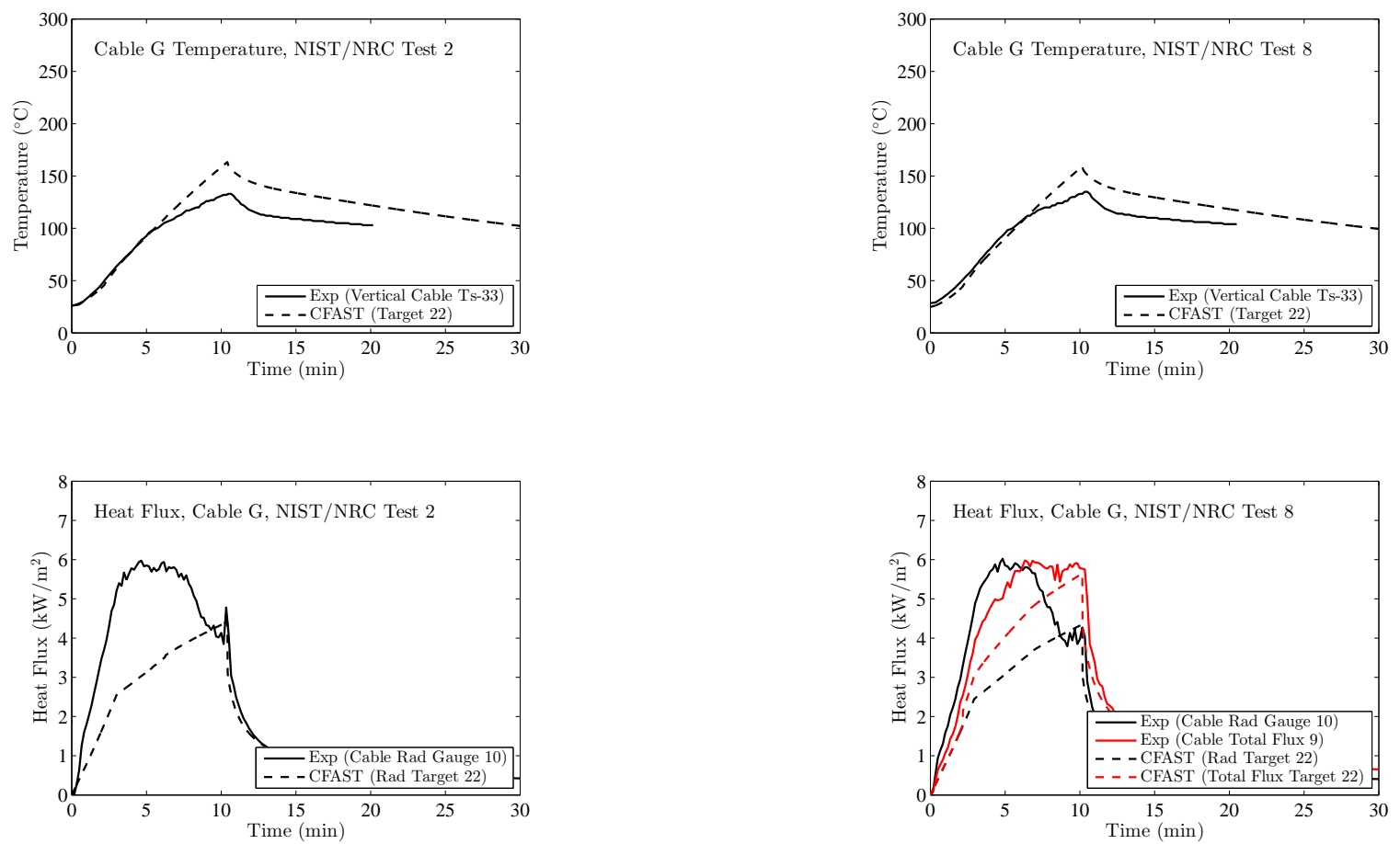

Figure B.42: NIST/NRC Series, Cable G Temperature and Heat Flux, Replicate Tests 2 and 8. 

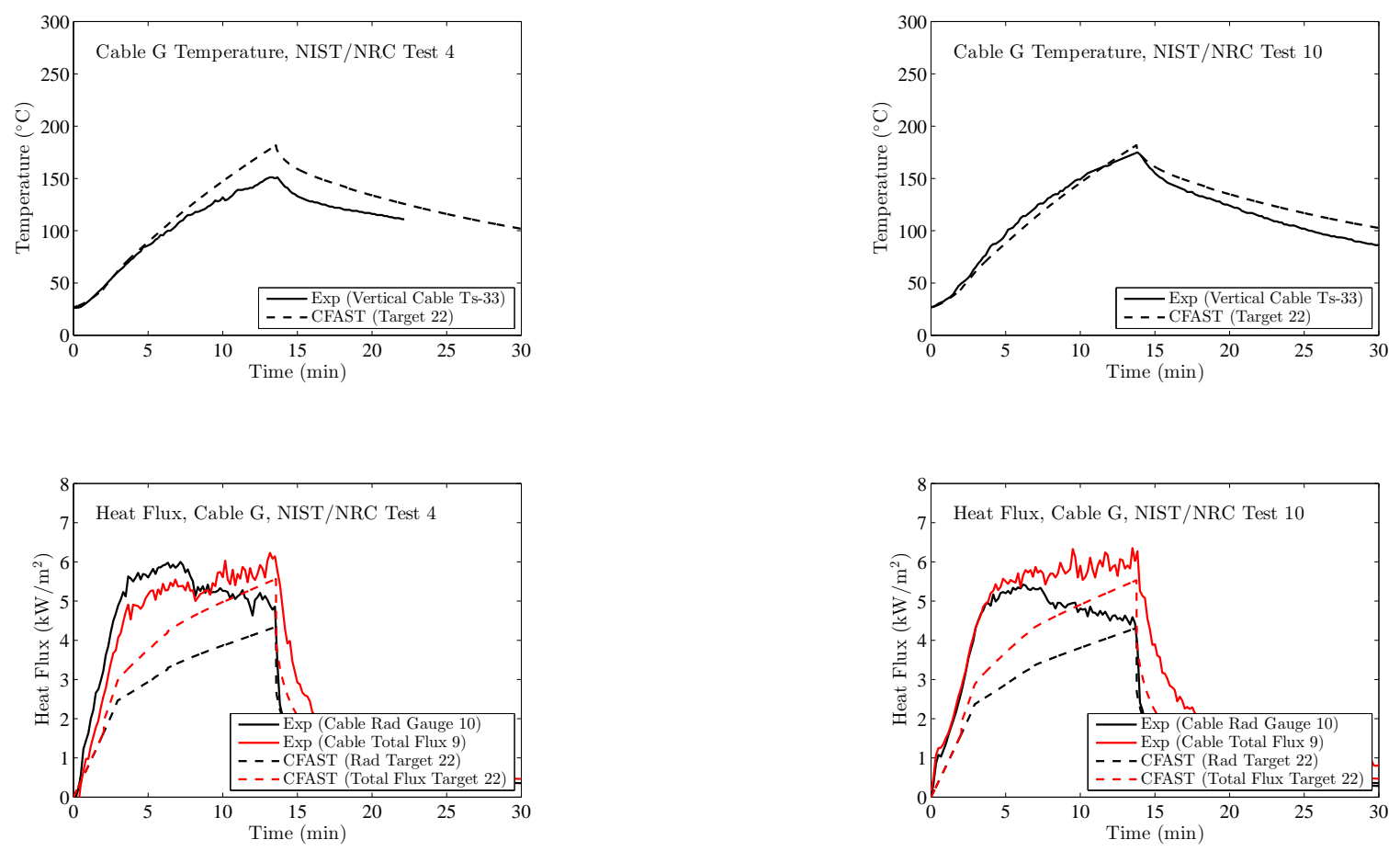

Figure B.43: NIST/NRC Series, Cable G Temperature and Heat Flux, Replicate Tests 4 and 10.
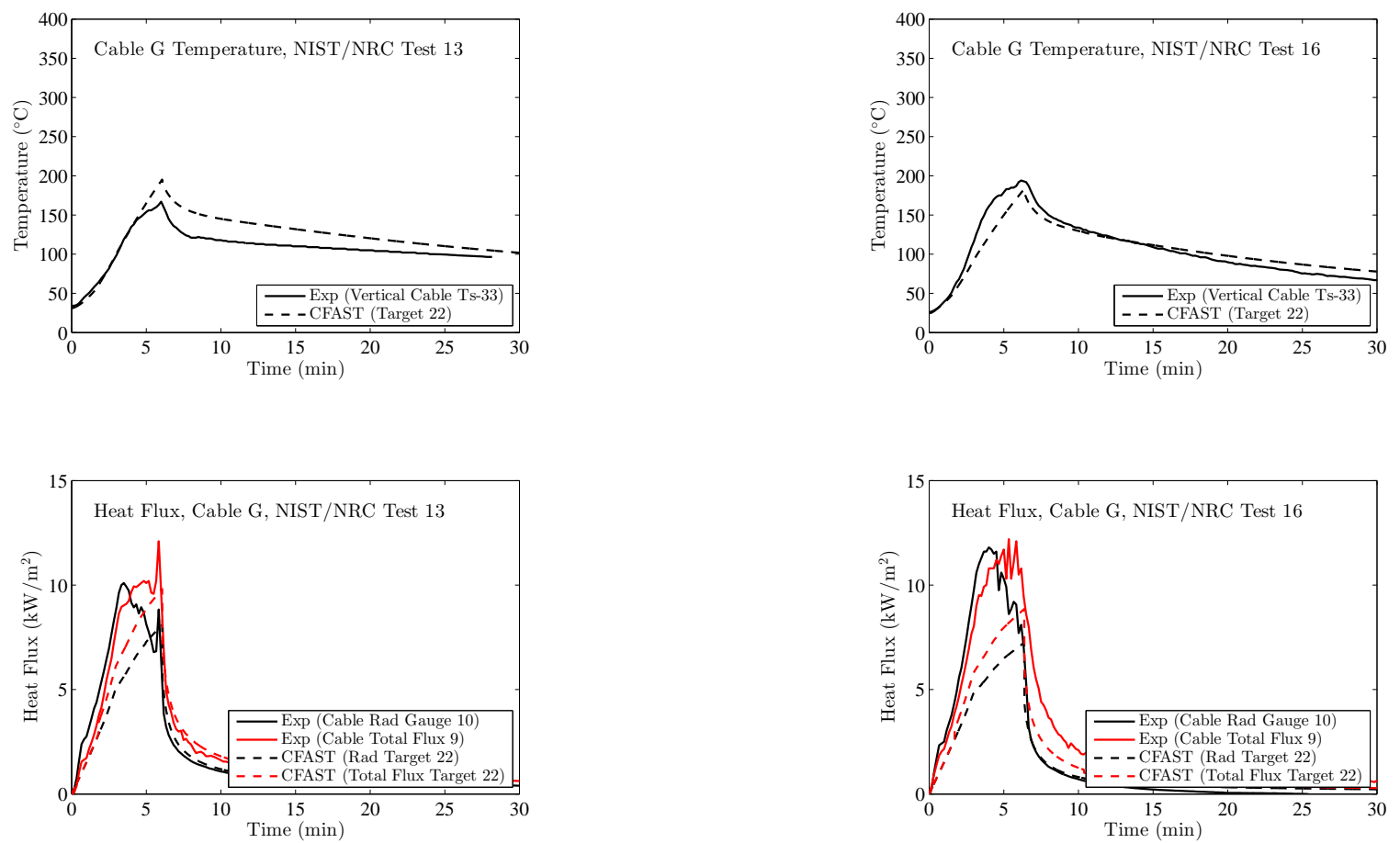

Figure B.44: NIST/NRC Series, Cable G Temperature and Heat Flux, Replicate Tests 13 and 16. 

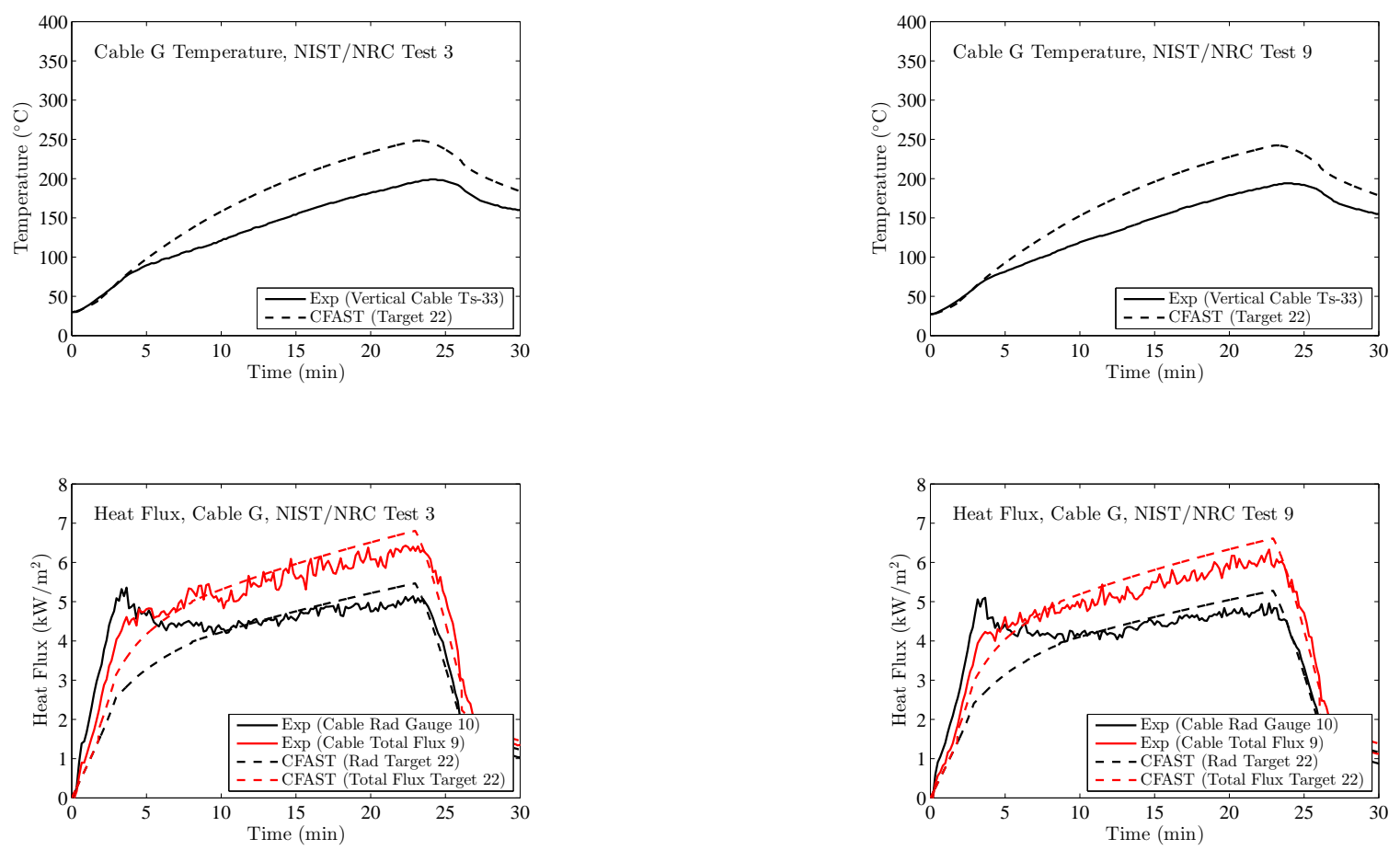

Figure B.45: NIST/NRC Series, Cable G Temperature and Heat Flux, Replicate Tests 3 and 9.
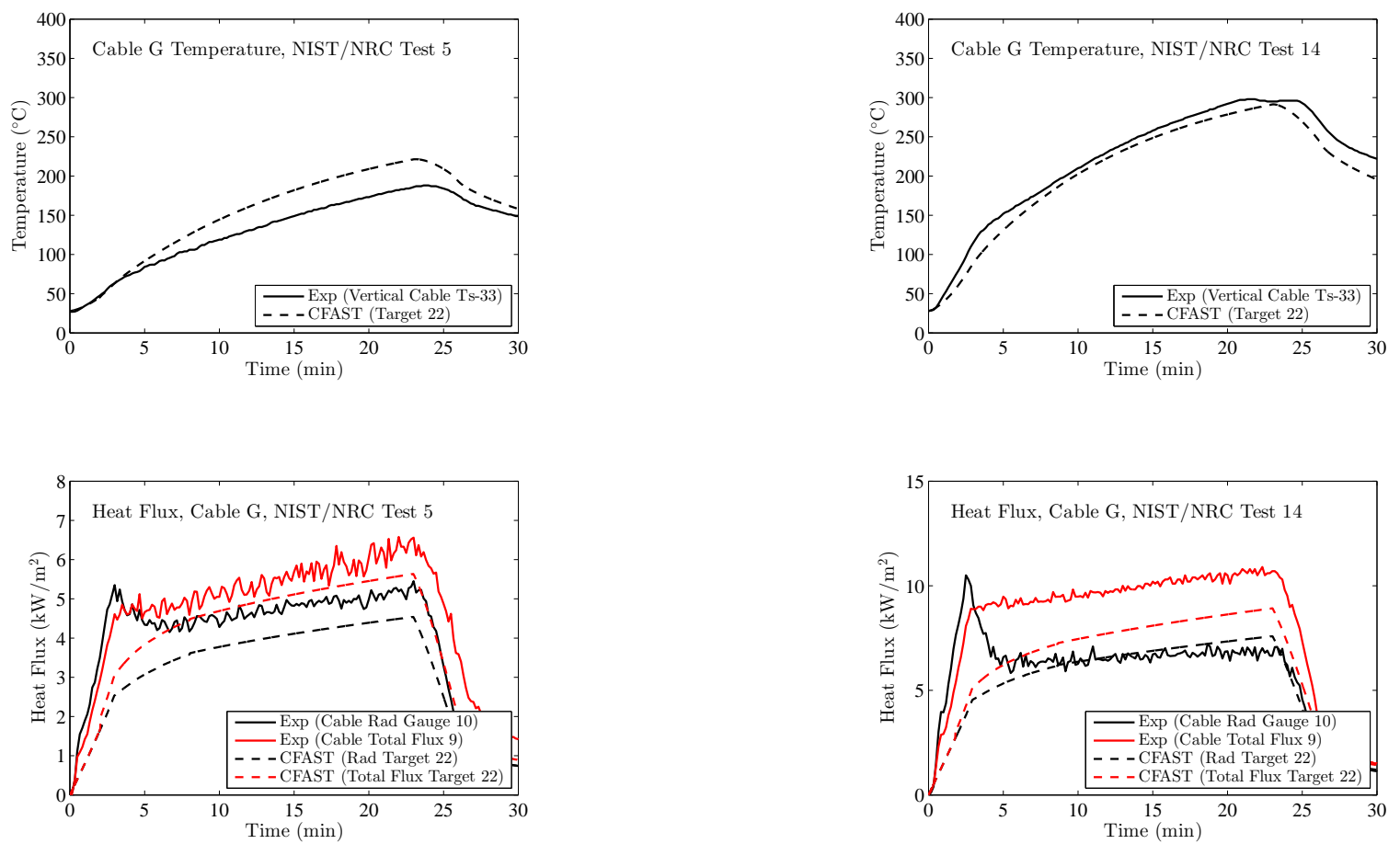

Figure B.46: NIST/NRC Series, Cable G Temperature and Heat Flux, Replicate Tests 5 and 14. 

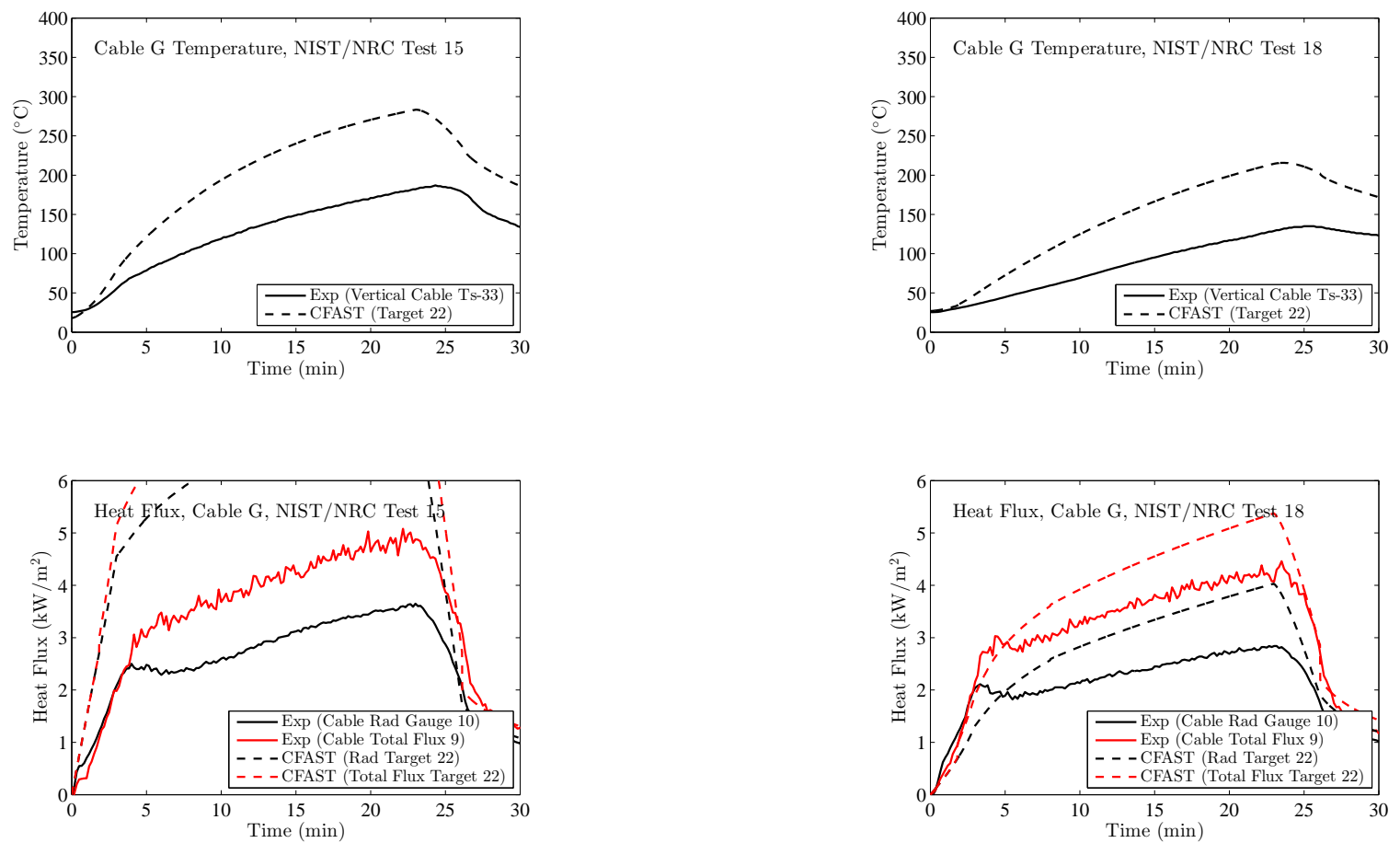

Figure B.47: NIST/NRC Series, Cable G Temperature and Heat Flux, Replicate Tests 15 and 18. 

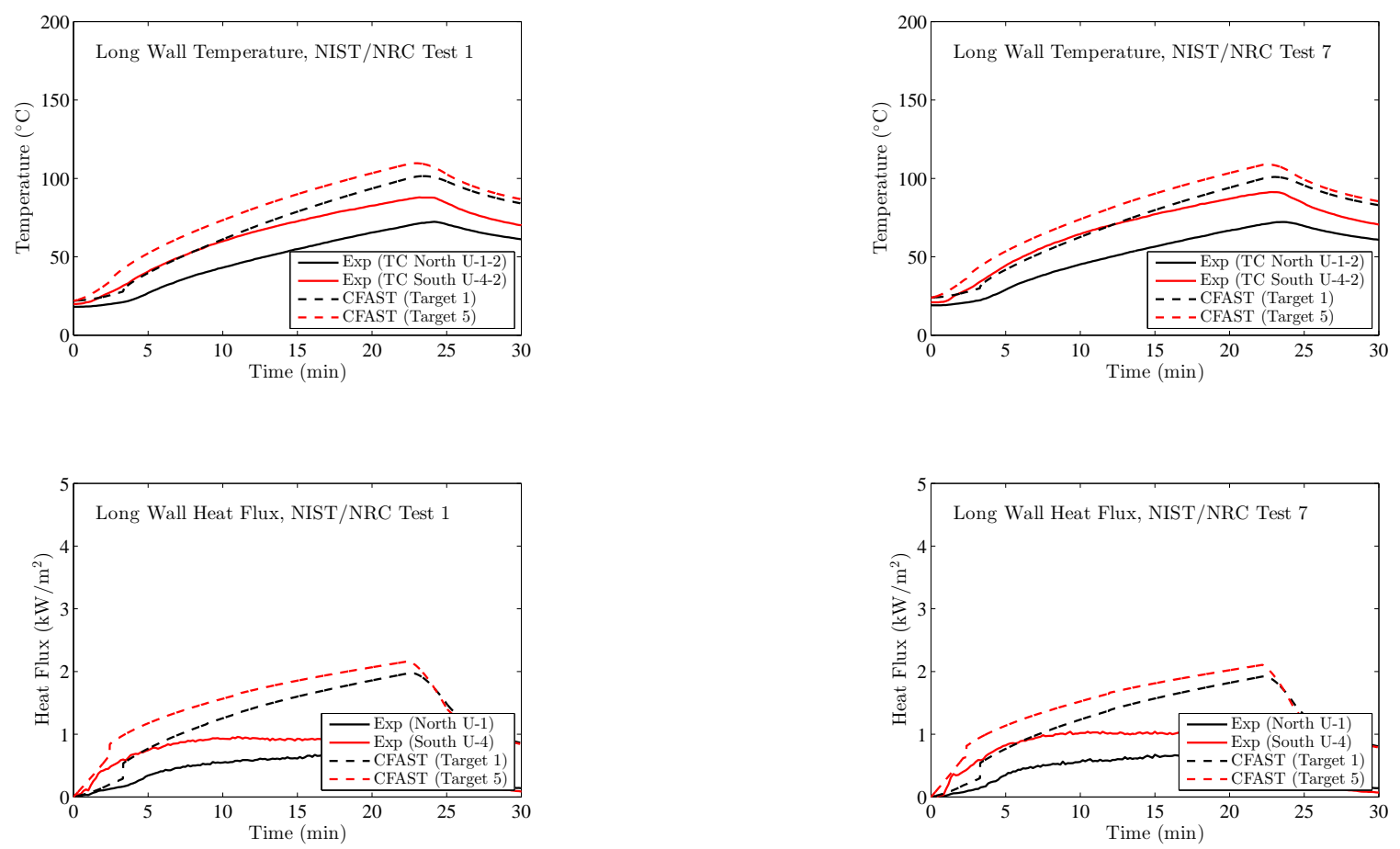

Figure B.48: NIST/NRC Series, Long Wall Temperature and Heat Flux, Replicate Tests 1 and 7.
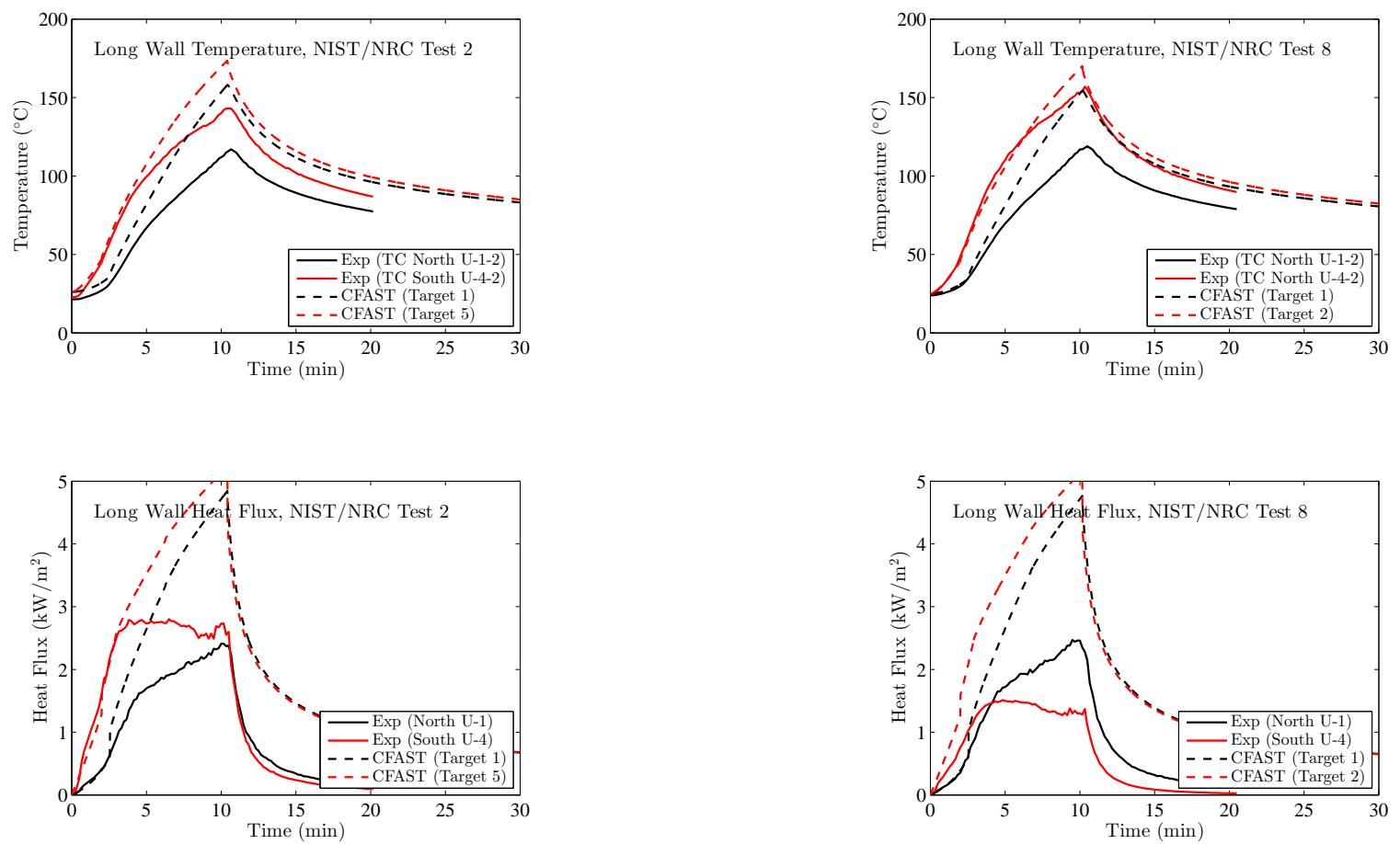

Figure B.49: NIST/NRC Series, Long Wall Temperature and Heat Flux, Replicate Tests 2 and 8. 

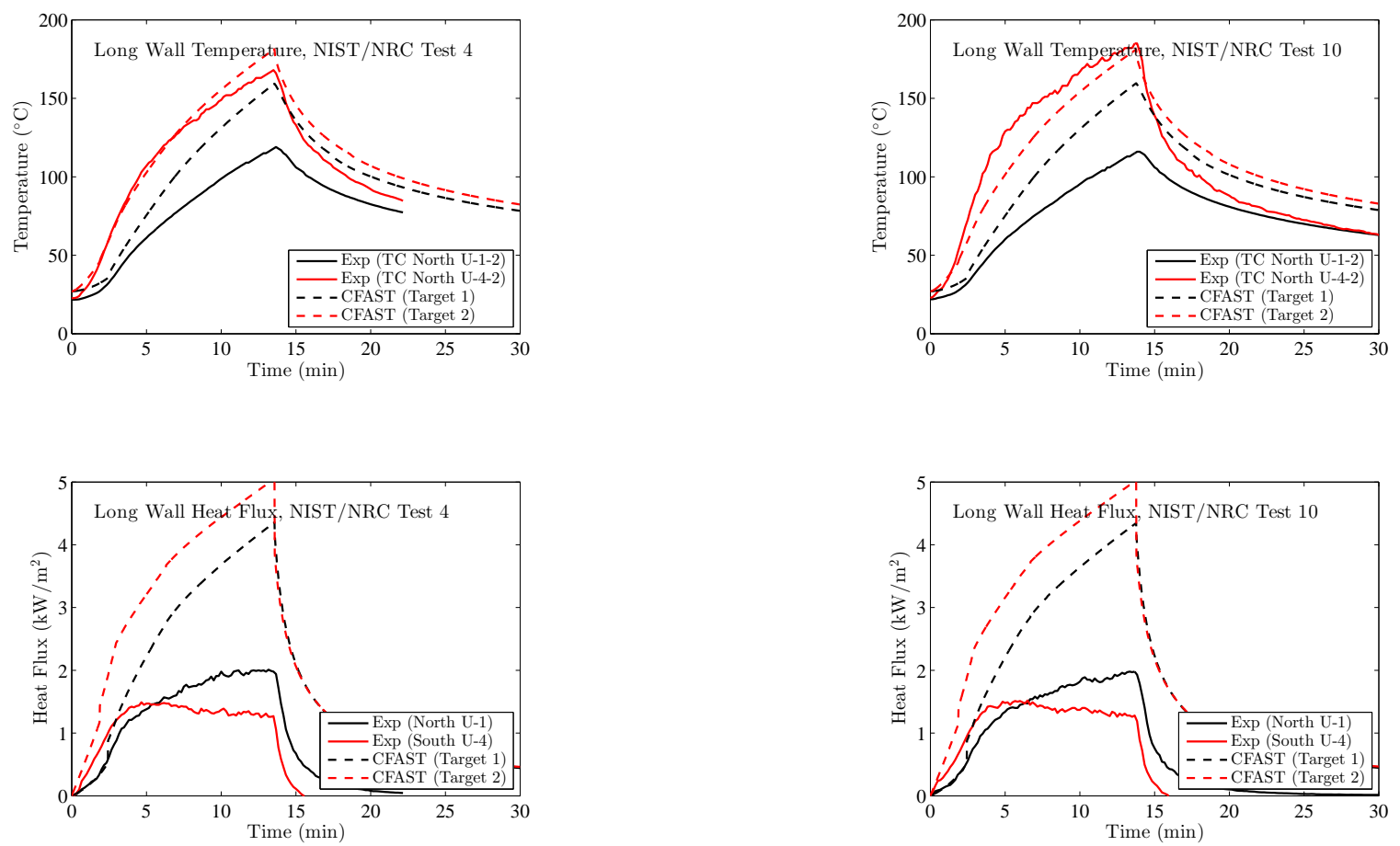

Figure B.50: NIST/NRC Series, Long Wall Temperature and Heat Flux, Replicate Tests 4 and 10.

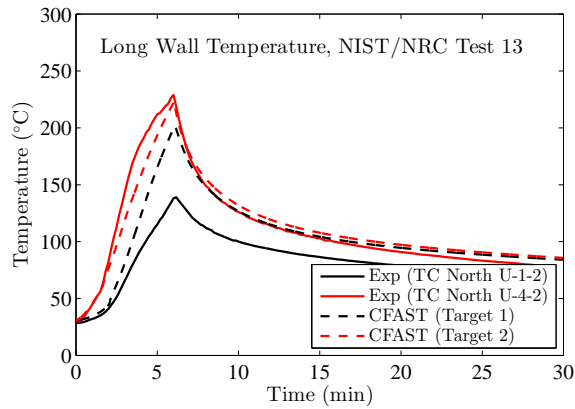

Experimental Heat Flux Data Not Available

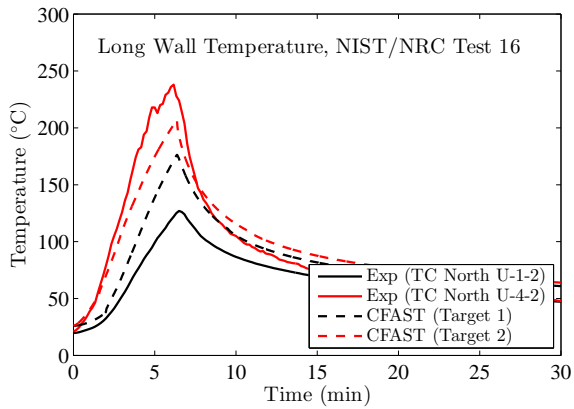

Experimental Heat Flux Data Not Available

Figure B.51: NIST/NRC Series, Long Wall Temperature and Heat Flux, Replicate Tests 13 and 16. 

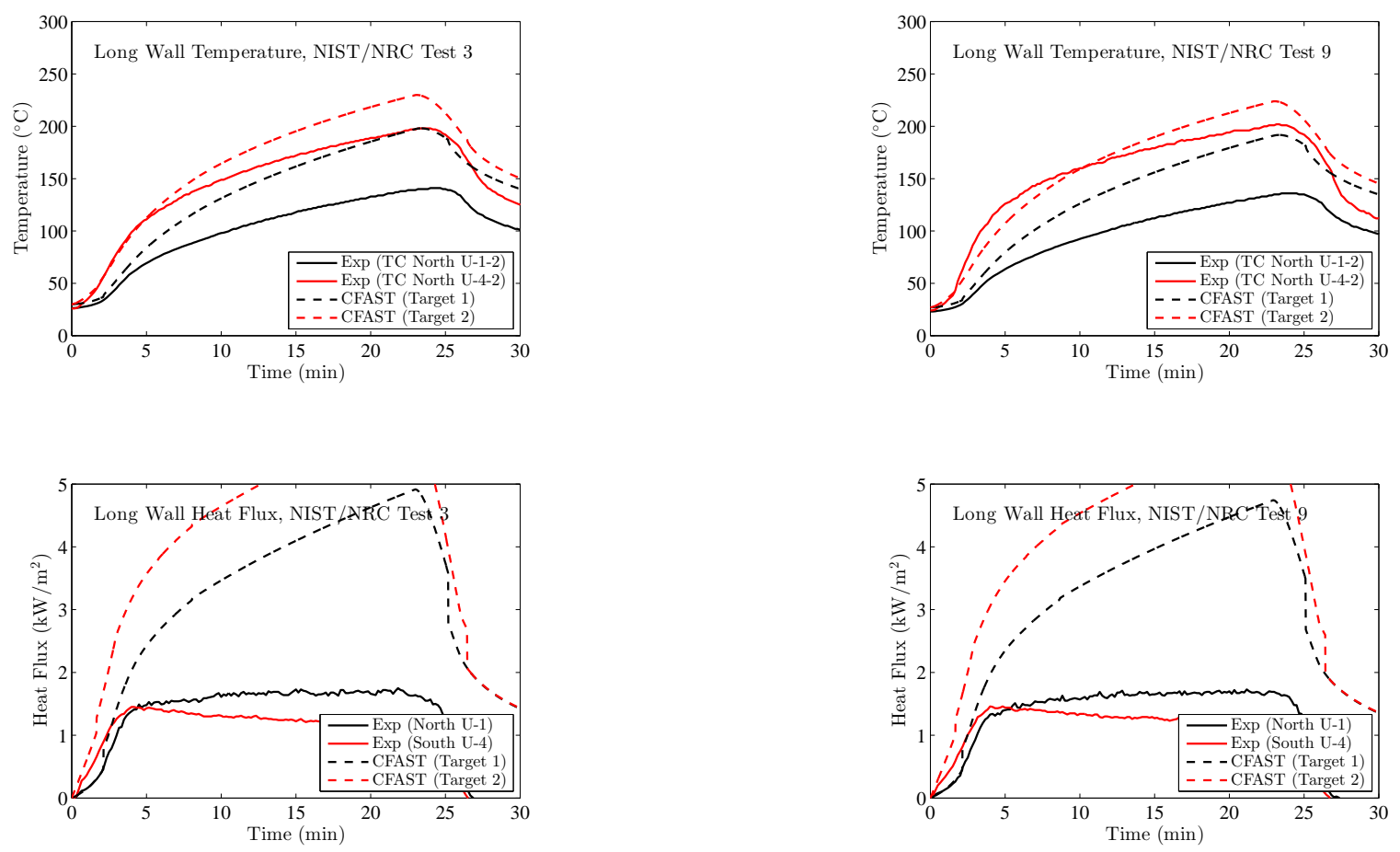

Figure B.52: NIST/NRC Series, Long Wall Temperature and Heat Flux, Replicate Tests 3 and 9.
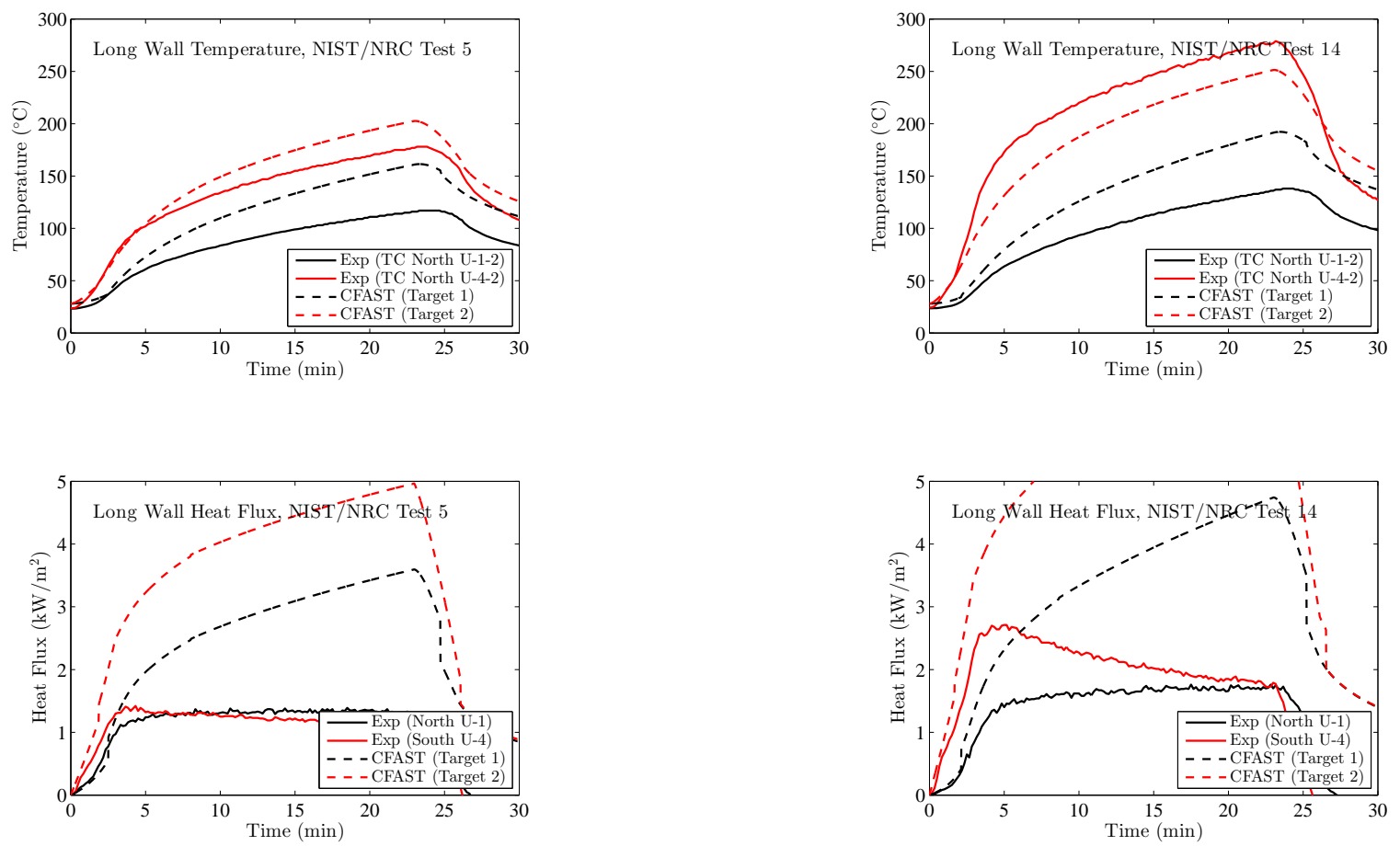

Figure B.53: NIST/NRC Series, Long Wall Temperature and Heat Flux, Replicate Tests 5 and 14. 

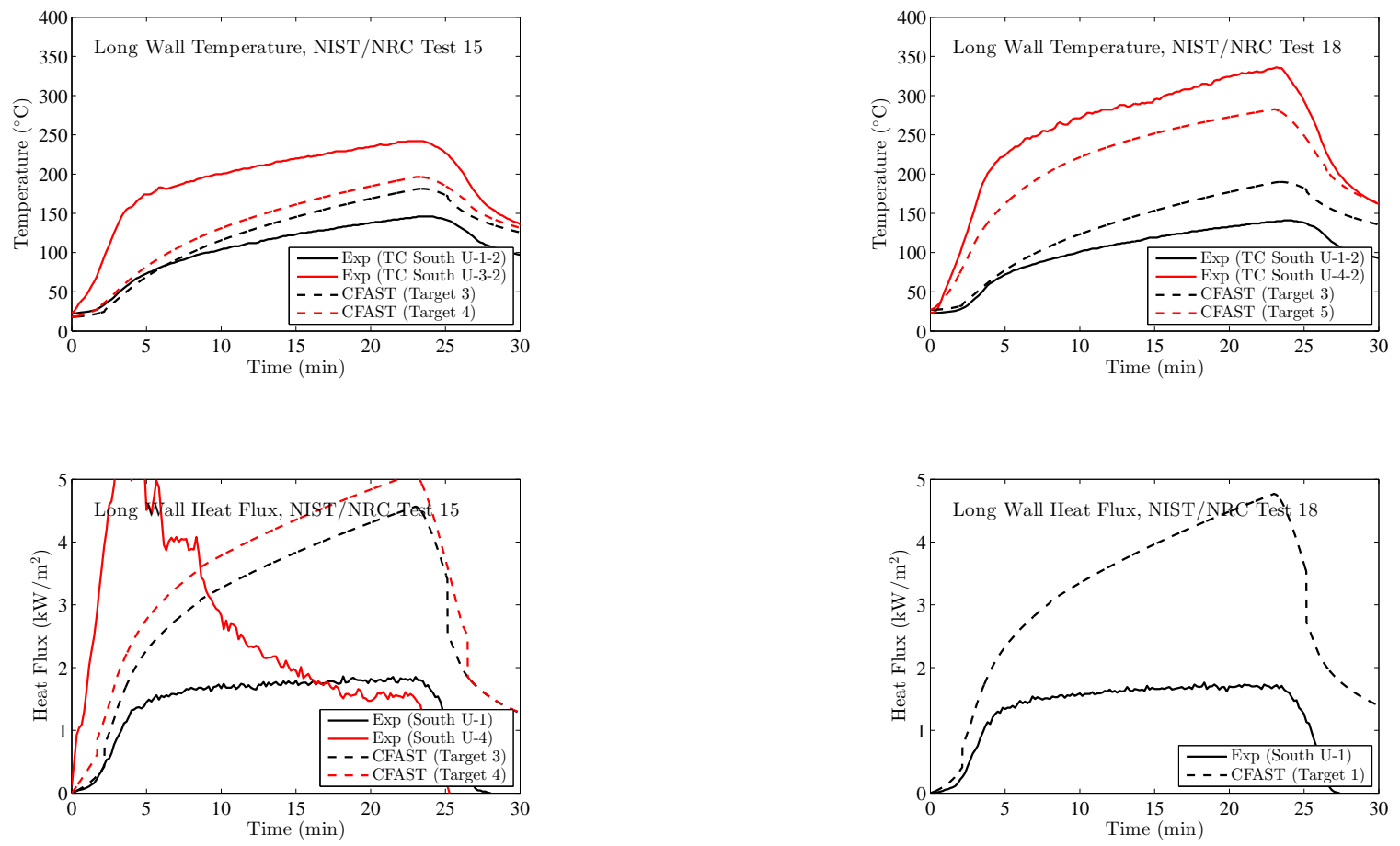

Figure B.54: NIST/NRC Series, Long Wall Temperature and Heat Flux, Replicate Tests 15 and 18. 

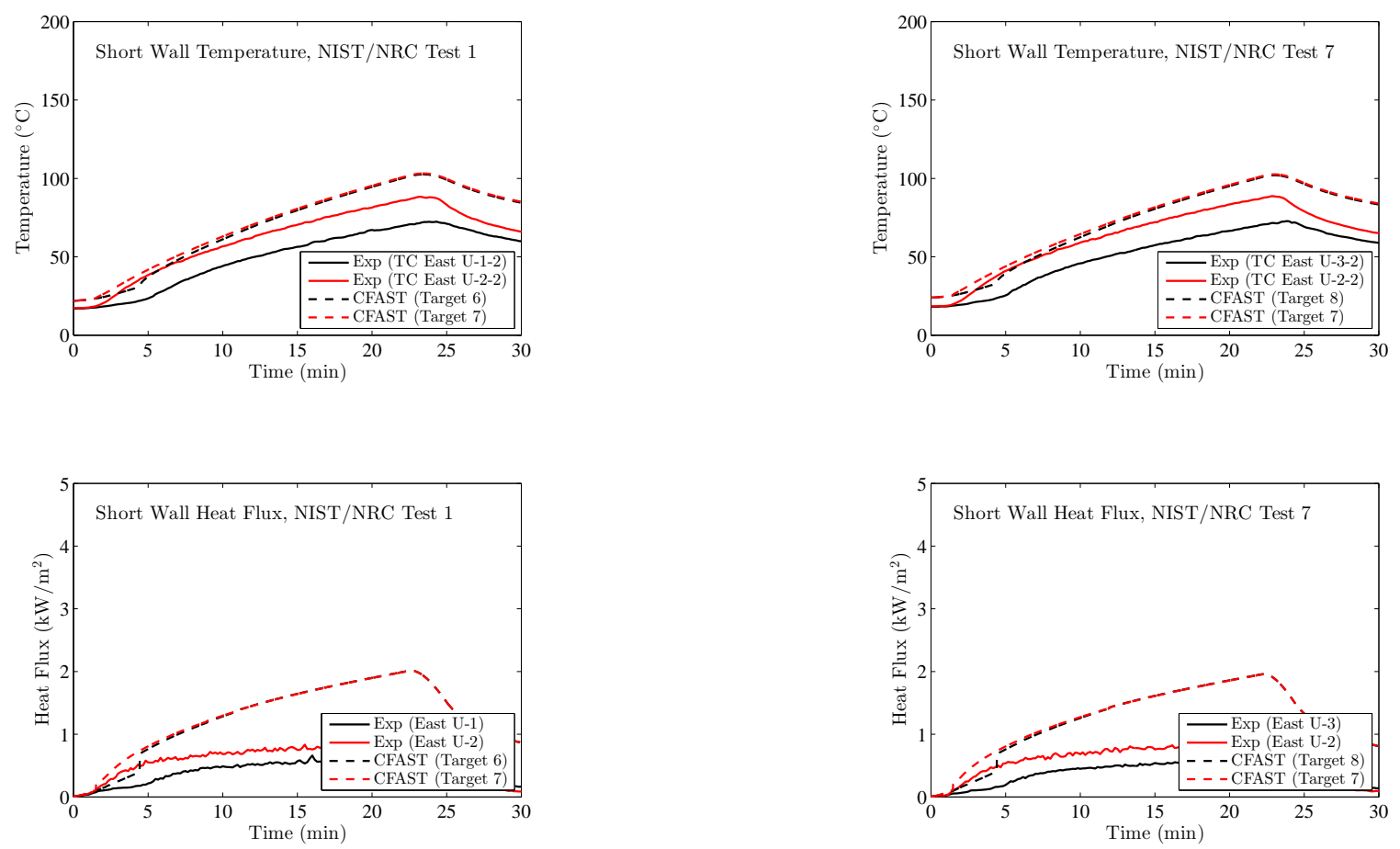

Figure B.55: NIST/NRC Series, Short Wall Temperature and Heat Flux, Replicate Tests 1 and 7.
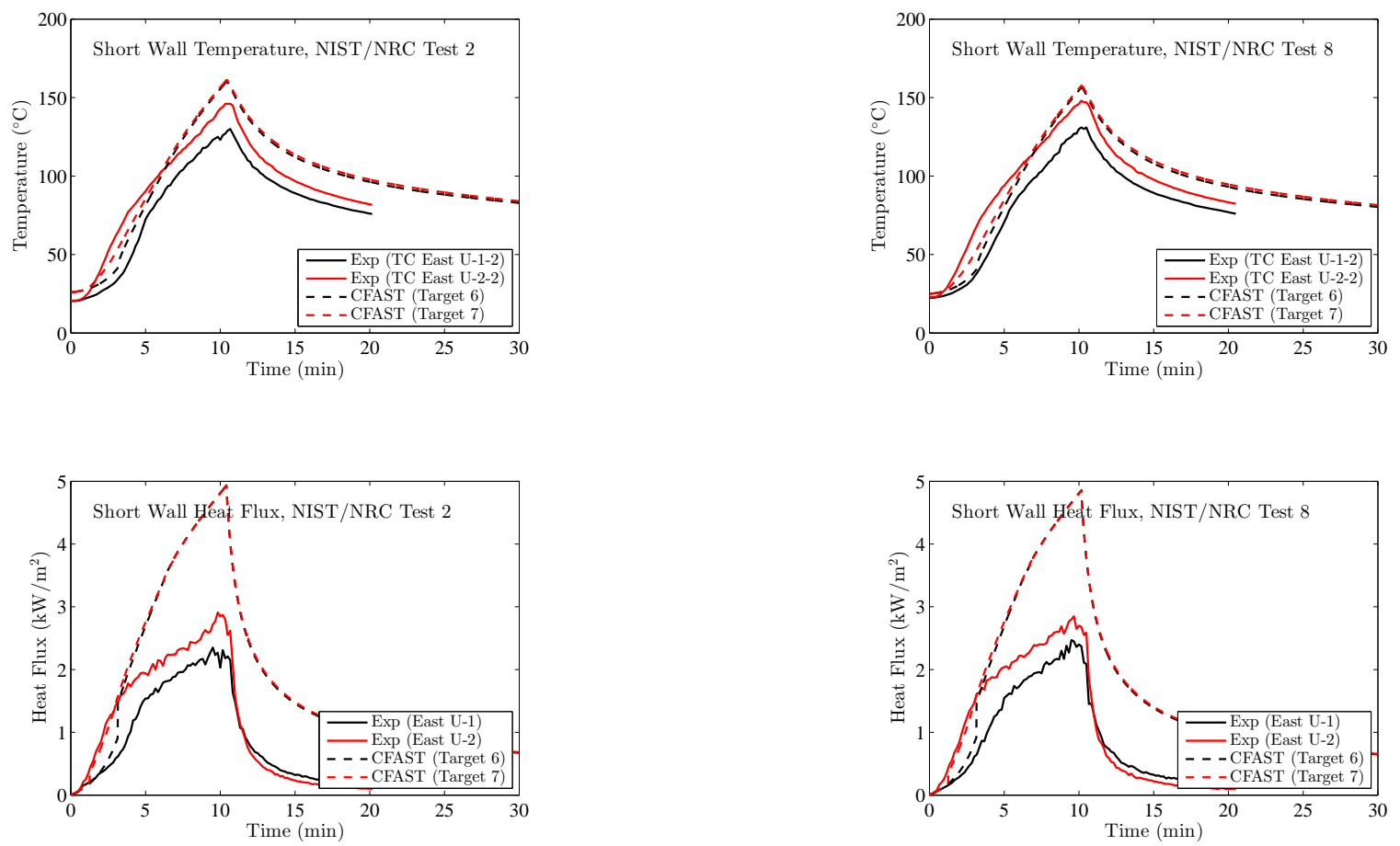

Figure B.56: NIST/NRC Series, Short Wall Temperature and Heat Flux, Replicate Tests 2 and 8. 

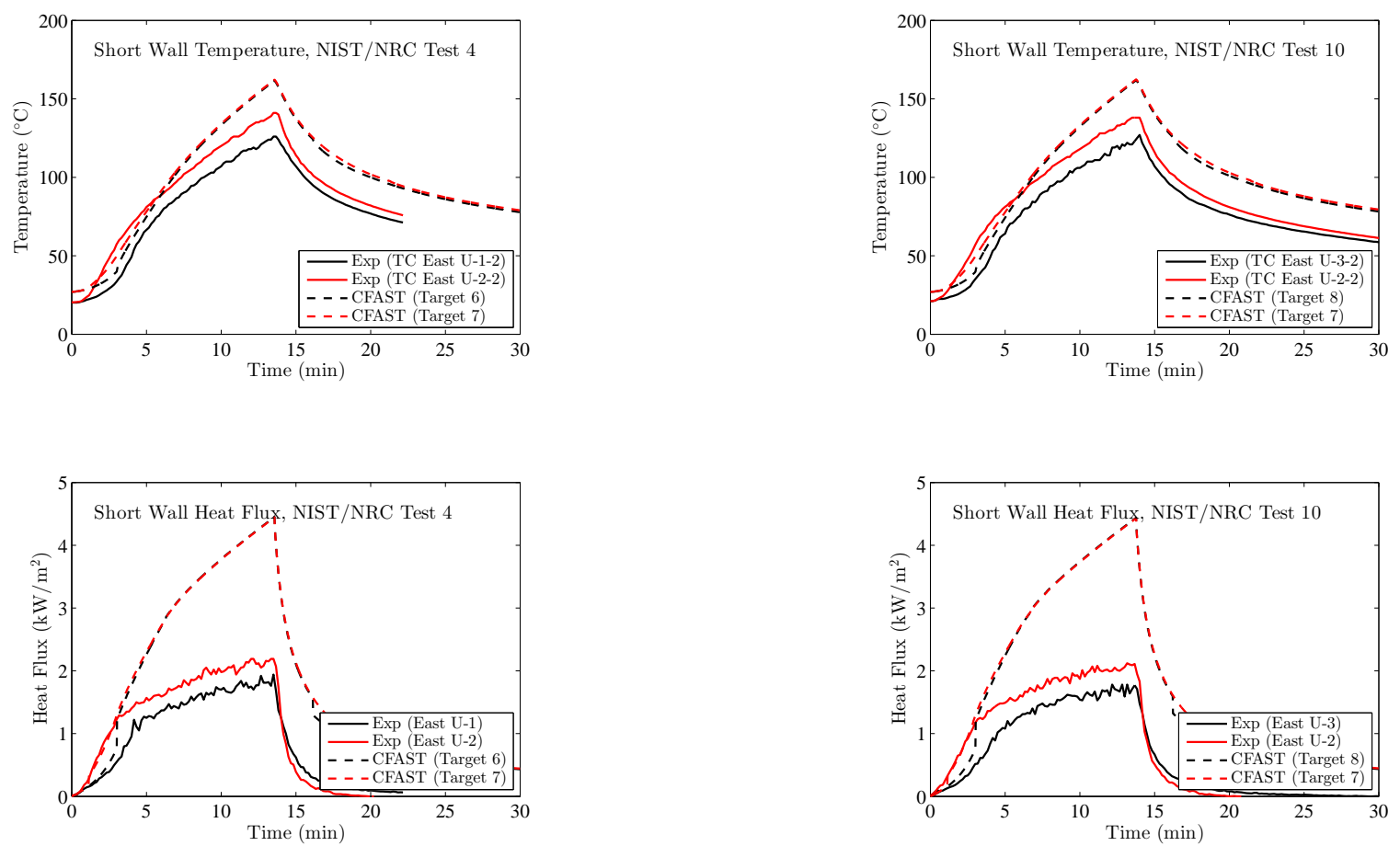

Figure B.57: NIST/NRC Series, Short Wall Temperature and Heat Flux, Replicate Tests 4 and 10.

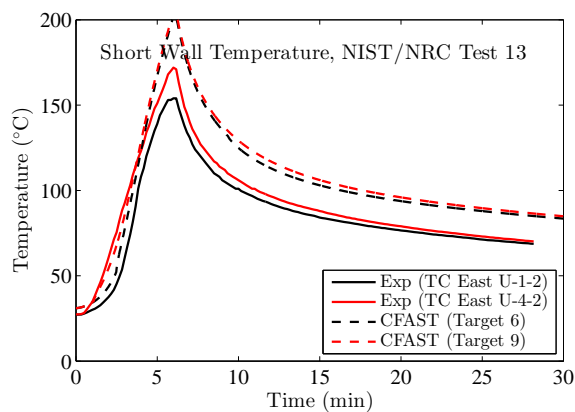

Experimental Heat Flux Data Not Available

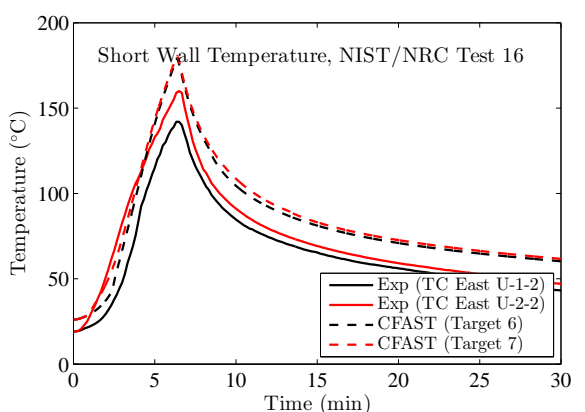

Experimental Heat Flux Data Not Available

Figure B.58: NIST/NRC Series, Short Wall Temperature and Heat Flux, Replicate Tests 13 and 16. 

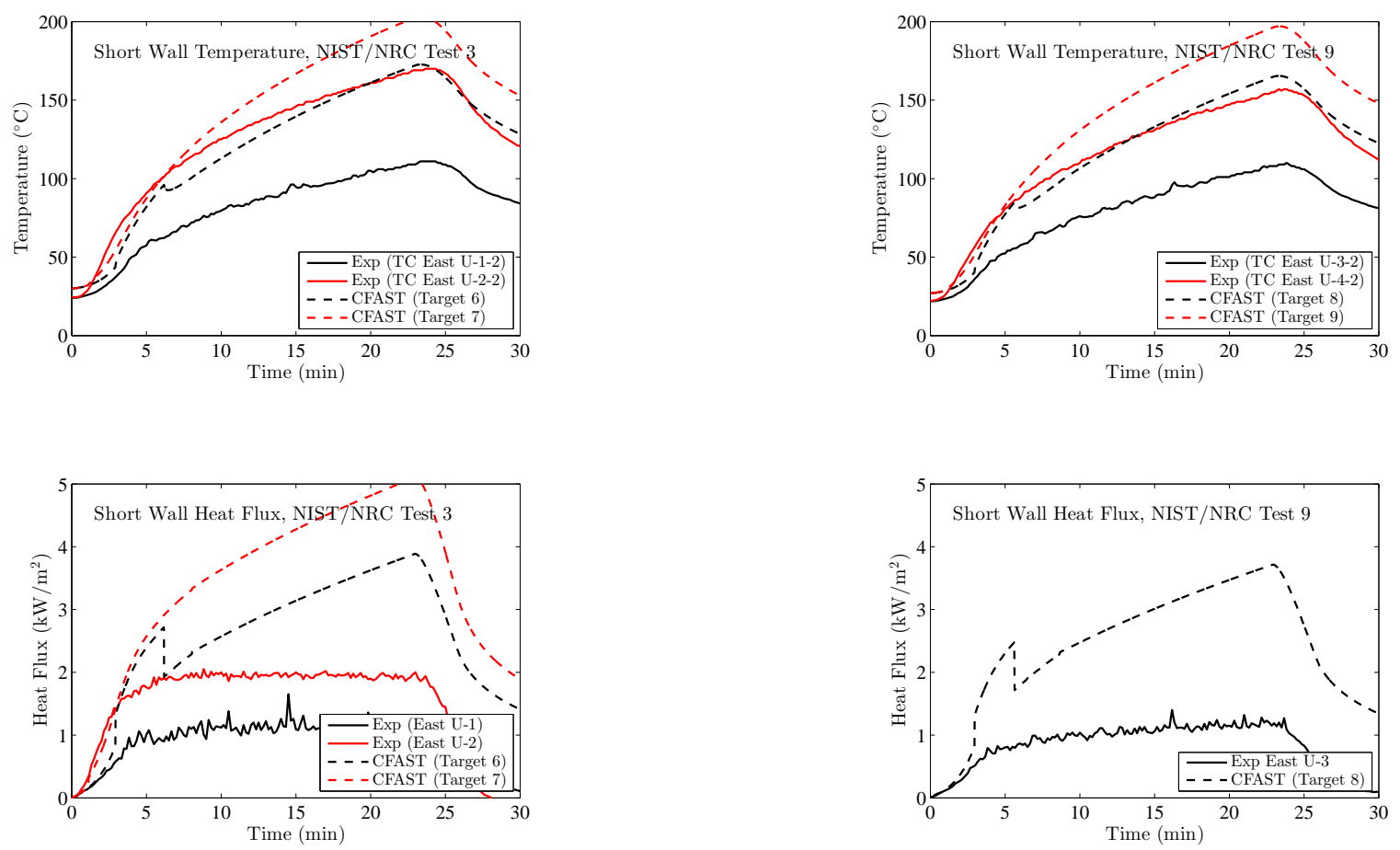

Figure B.59: NIST/NRC Series, Short Wall Temperature and Heat Flux, Replicate Tests 3 and 9.
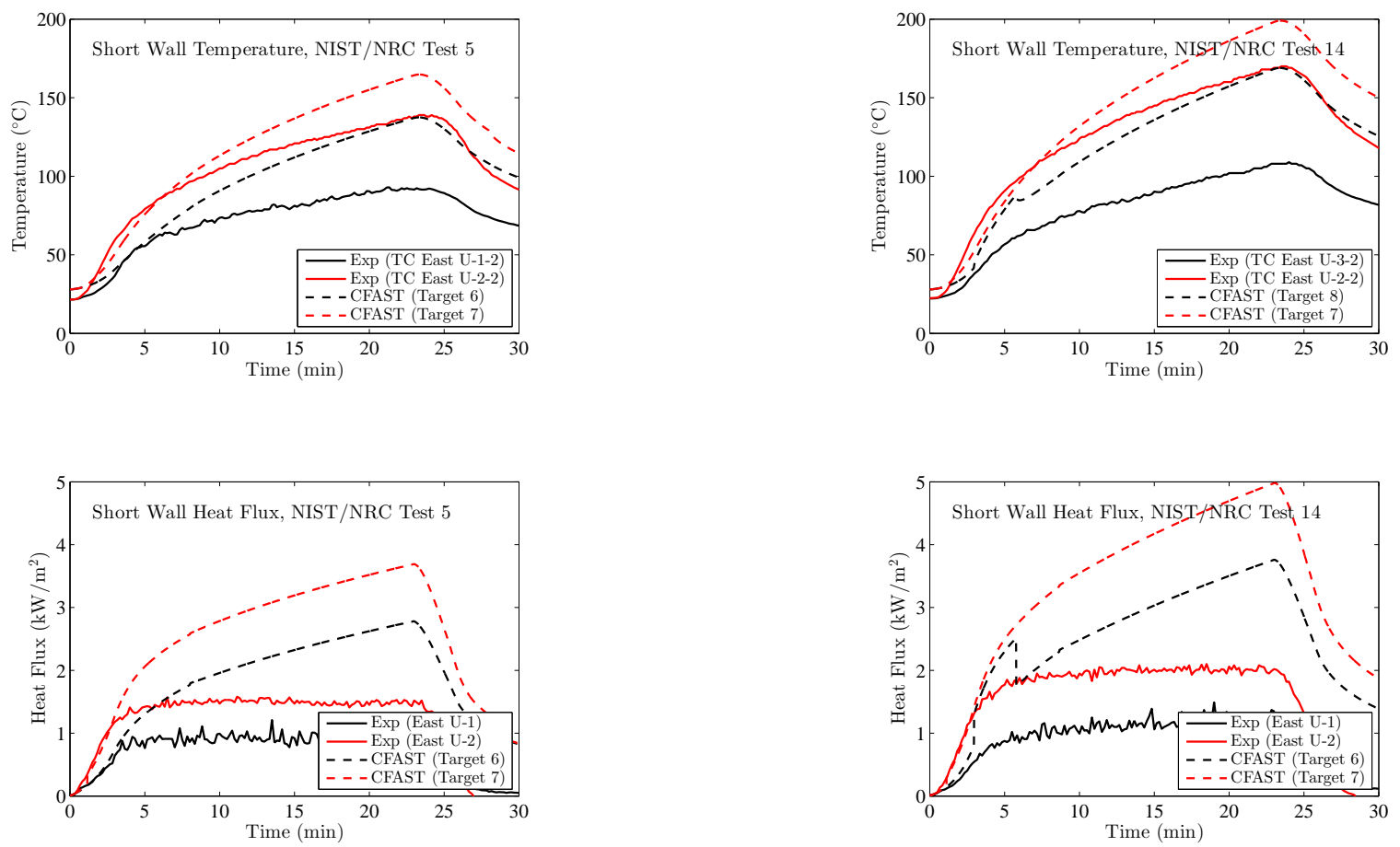

Figure B.60: NIST/NRC Series, Short Wall Temperature and Heat Flux, Replicate Tests 5 and 14. 

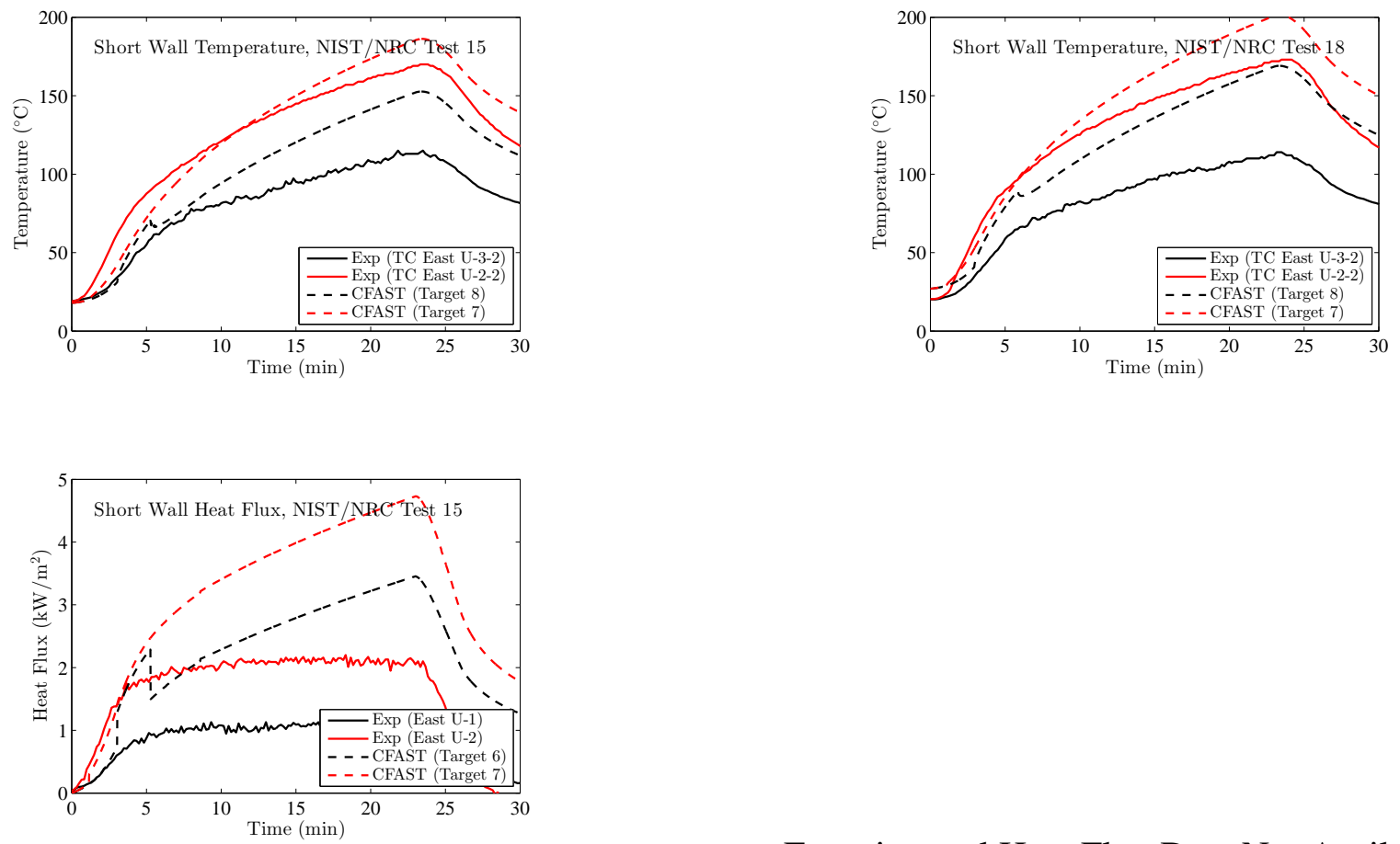

Experimental Heat Flux Data Not Available

Figure B.61: NIST/NRC Series, Short Wall Temperature and Heat Flux, Replicate Tests 15 and 18. 

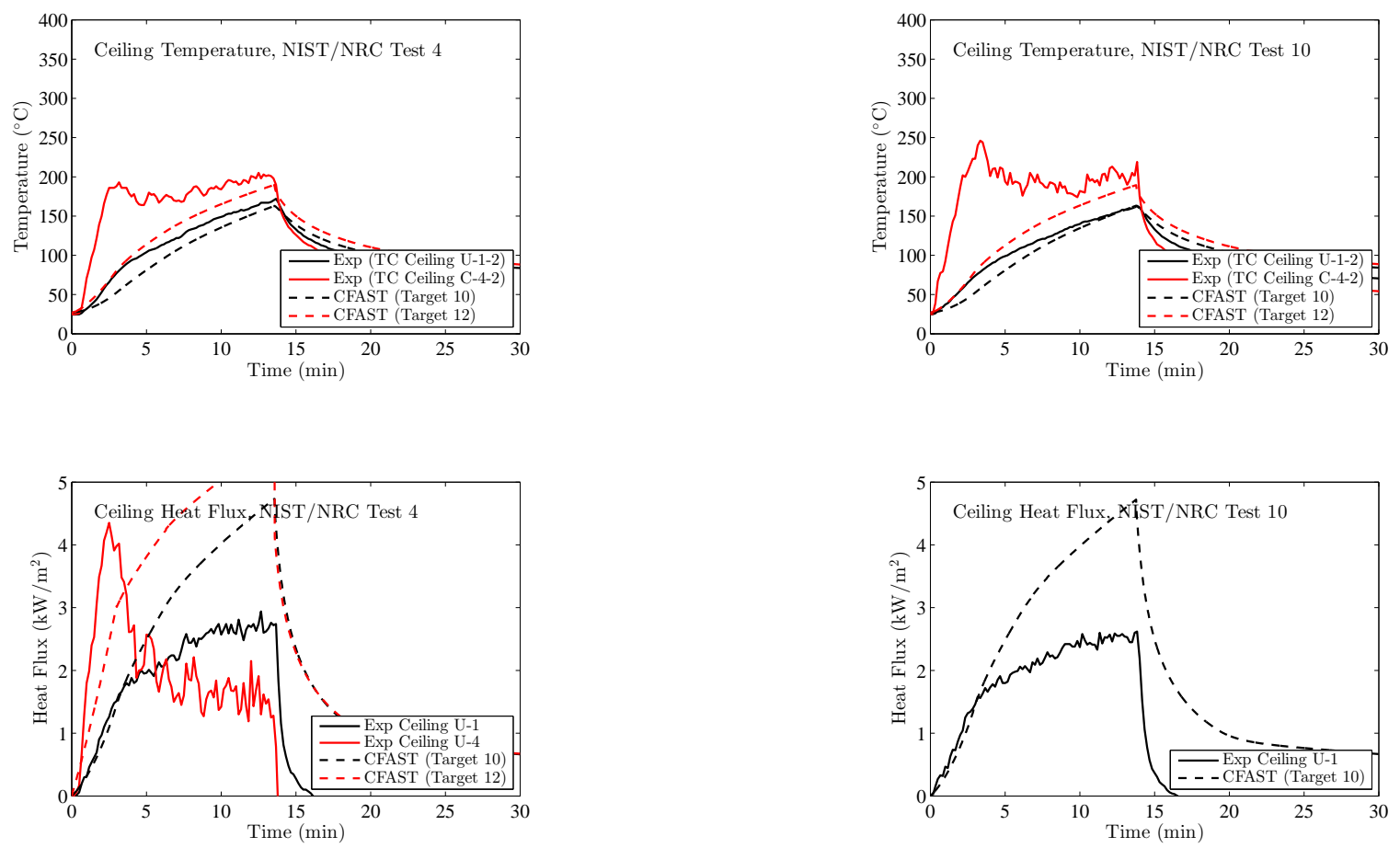

Figure B.62: NIST/NRC Series, Ceiling Temperature and Heat Flux, Replicate Tests 4 and 10.

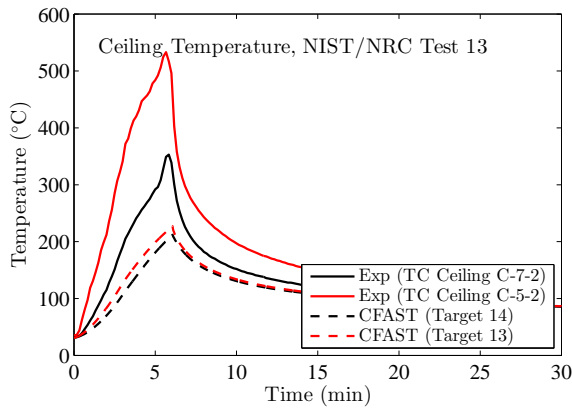

Experimental Heat Flux Data Not Available

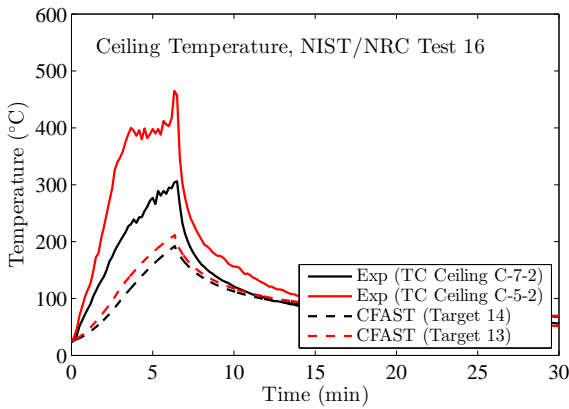

Experimental Heat Flux Data Not Available

Figure B.63: NIST/NRC Series, Ceiling Temperature and Heat Flux, Replicate Tests 13 and 16. 

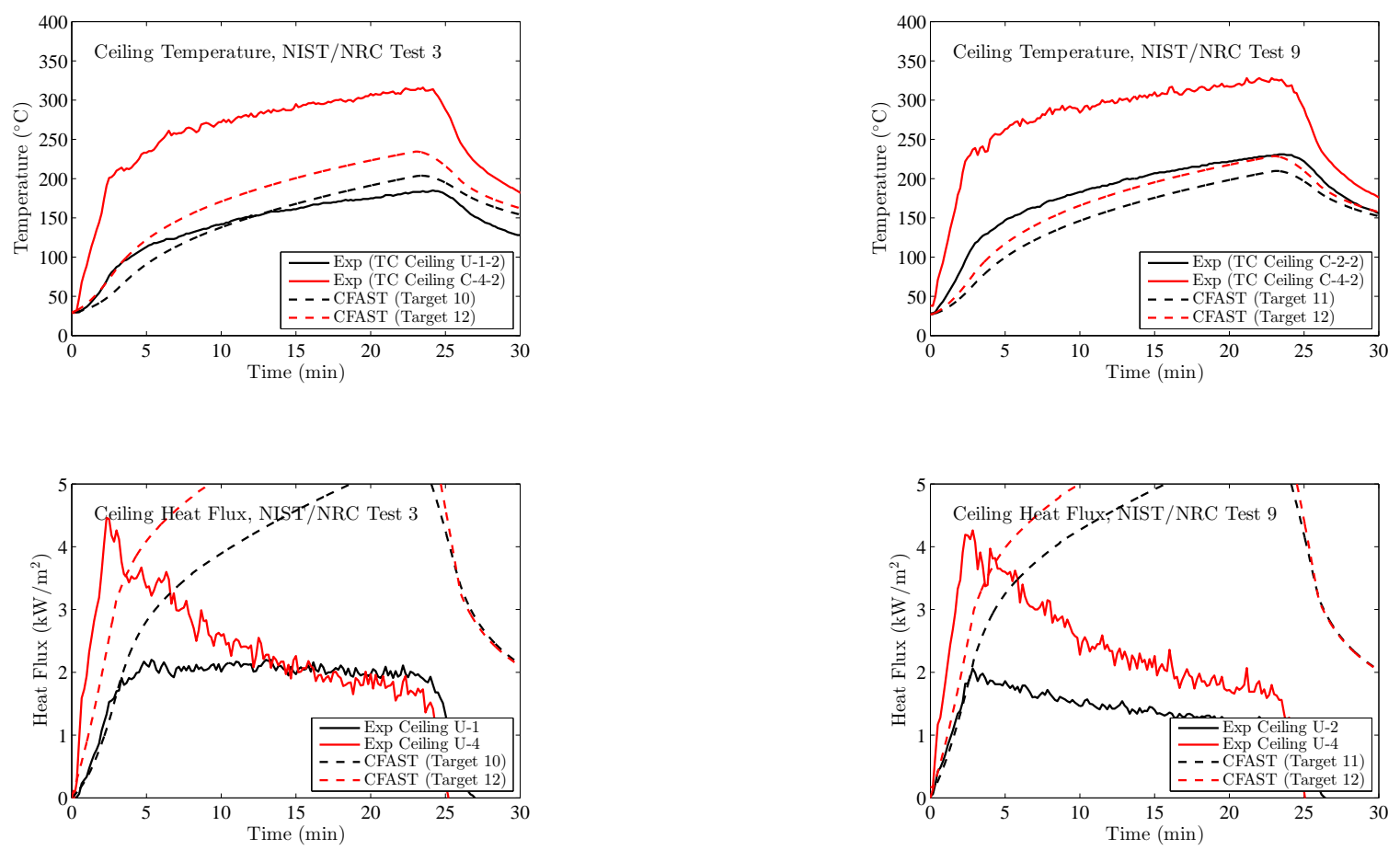

Figure B.64: NIST/NRC Series, Ceiling Temperature and Heat Flux, Replicate Tests 3 and 9.
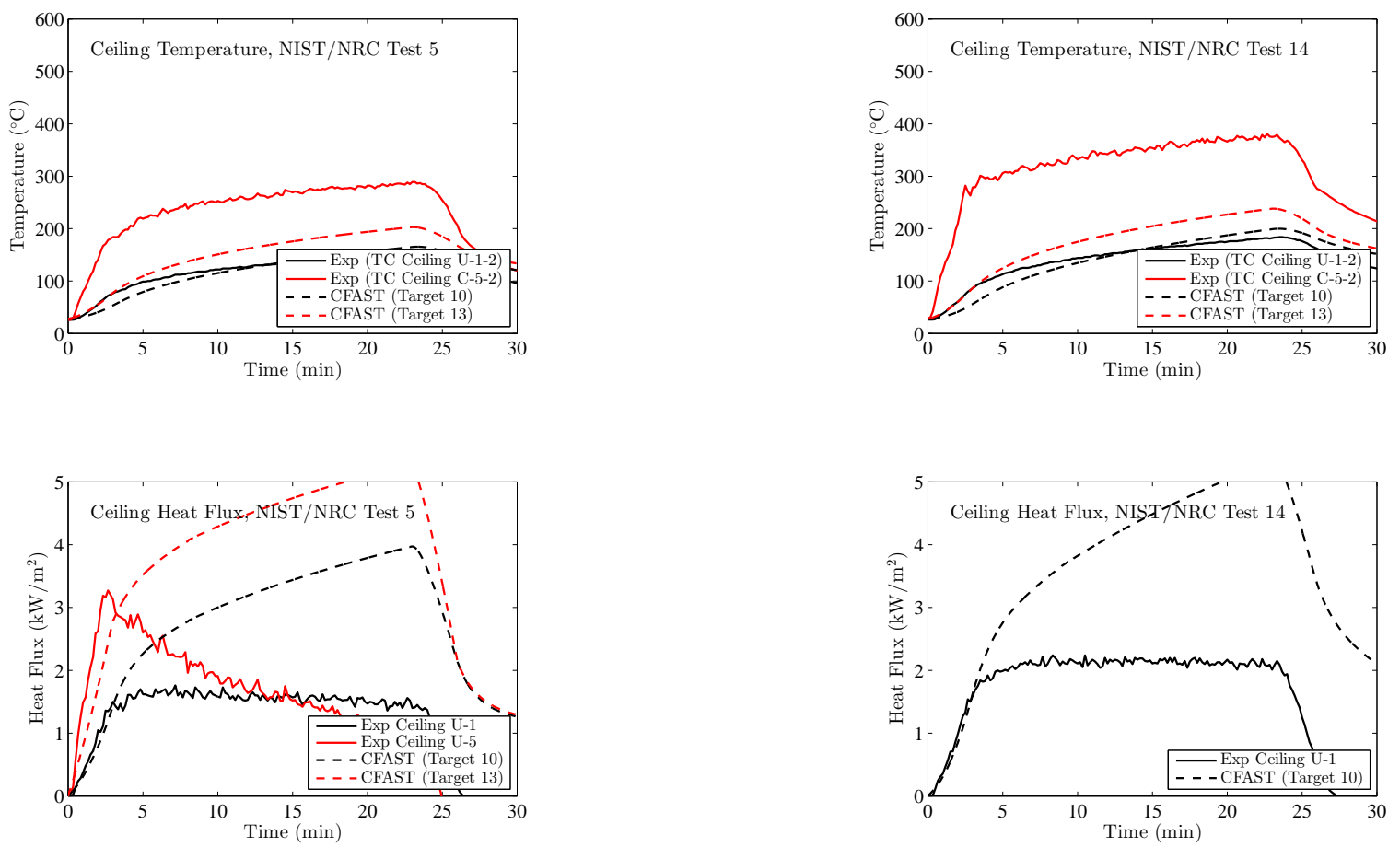

Figure B.65: NIST/NRC Series, Ceiling Temperature and Heat Flux, Replicate Tests 5 and 14. 

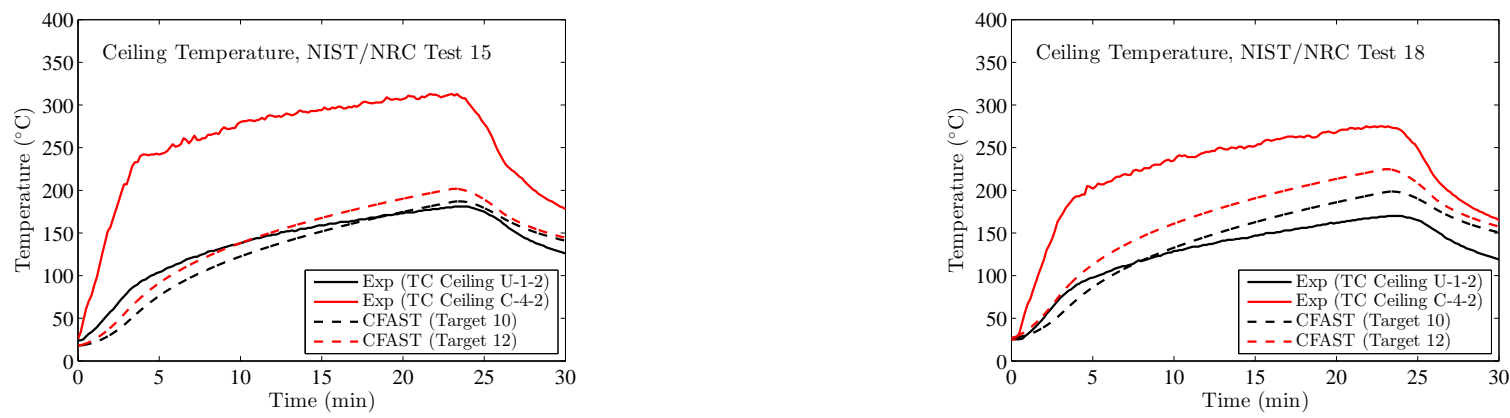

Experimental Heat Flux Data Not Available

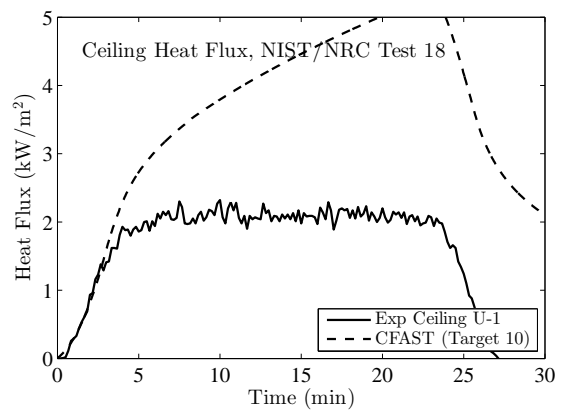

Figure B.66: NIST/NRC Series, Ceiling Temperature and Heat Flux, Replicate Tests 15 and 18. 

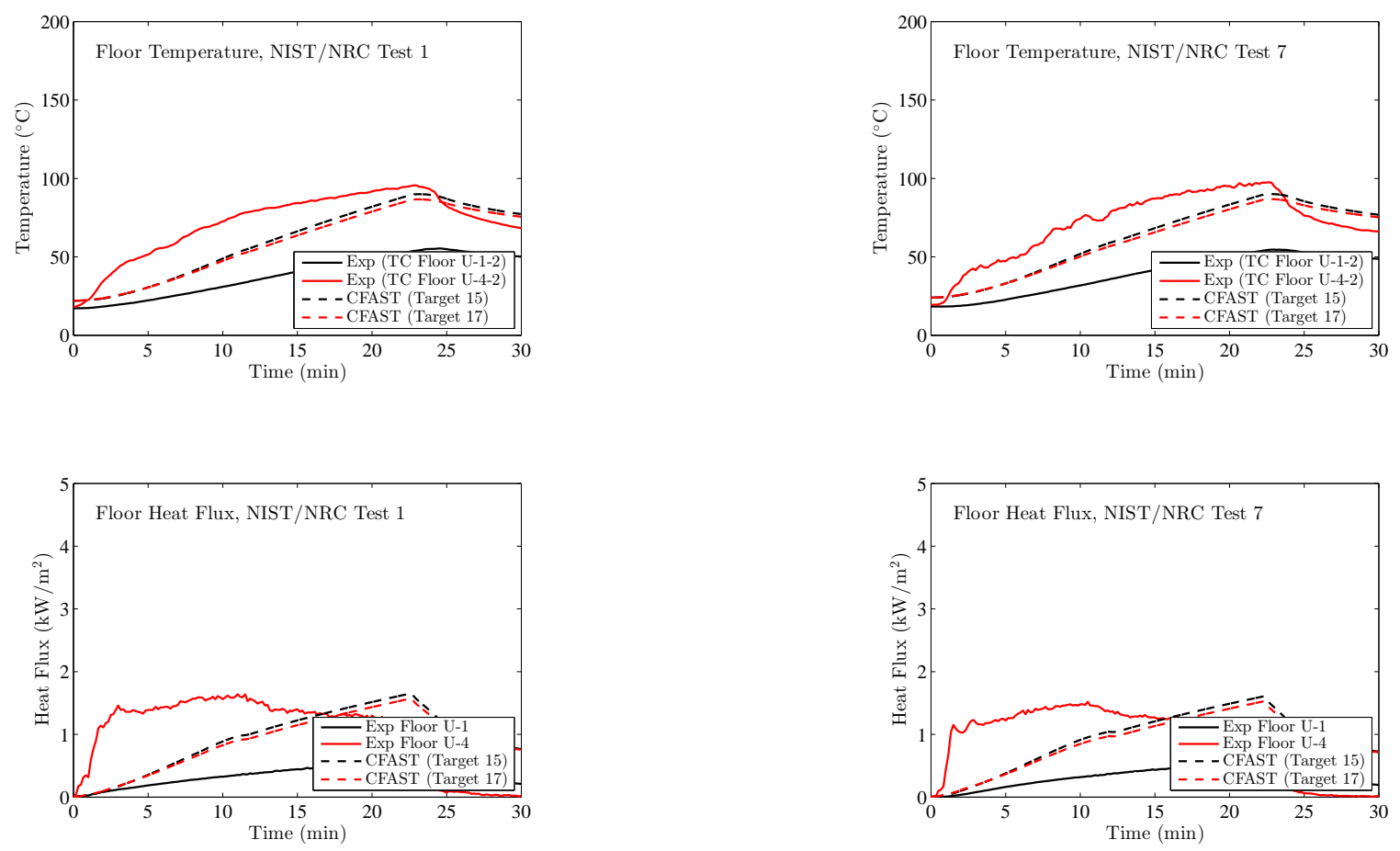

Figure B.67: NIST/NRC Series, Floor Temperature and Heat Flux, Replicate Tests 1 and 7.
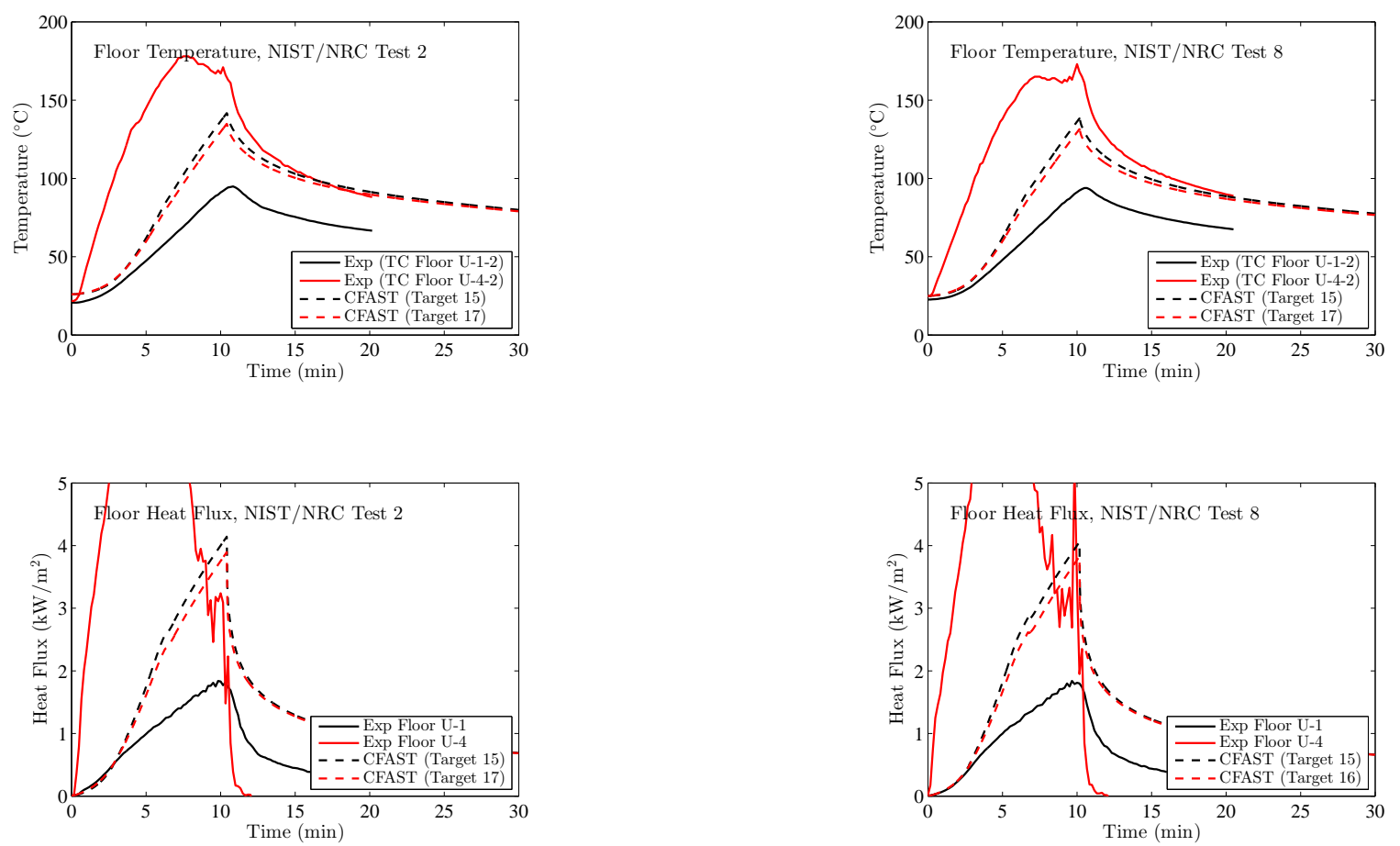

Figure B.68: NIST/NRC Series, Floor Temperature and Heat Flux, Replicate Tests 2 and 8. 

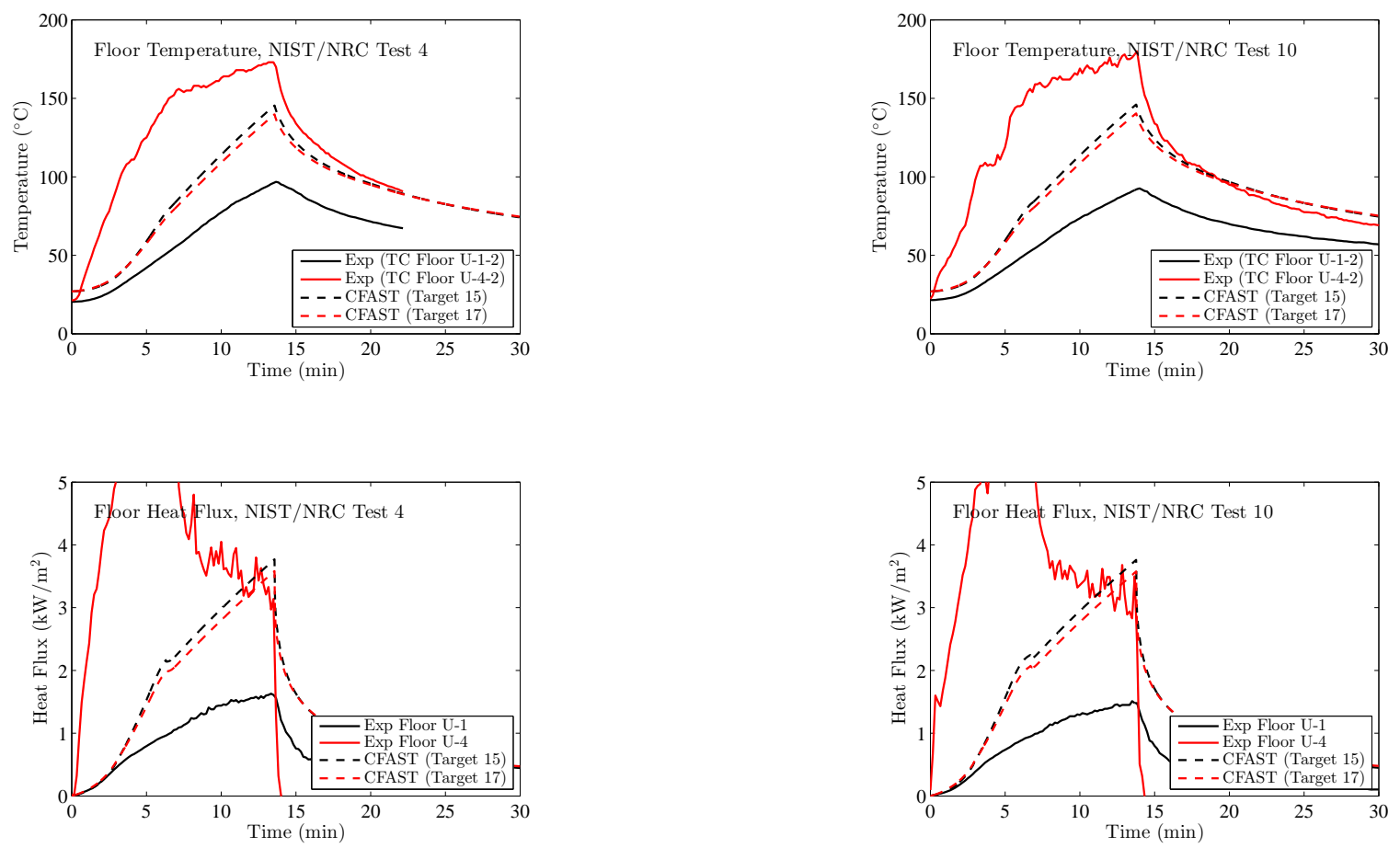

Figure B.69: NIST/NRC Series, Floor Temperature and Heat Flux, Replicate Tests 4 and 10.

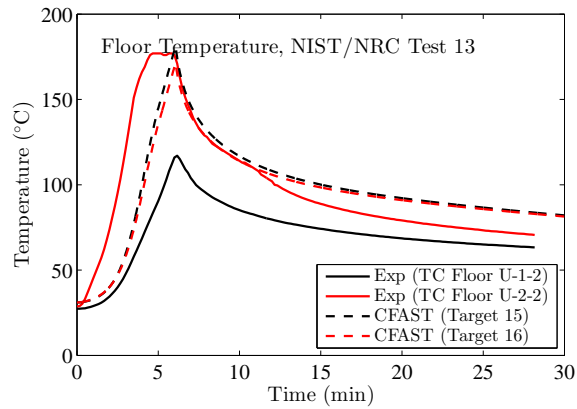

Experimental Heat Flux Data Not Available

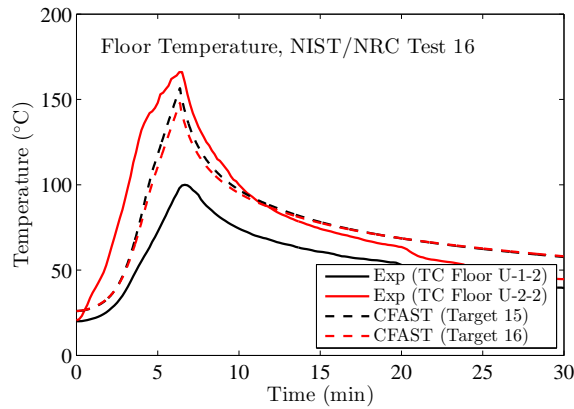

Experimental Heat Flux Data Not Available

Figure B.70: NIST/NRC Series, Floor Temperature and Heat Flux, Replicate Tests 13 and 16. 

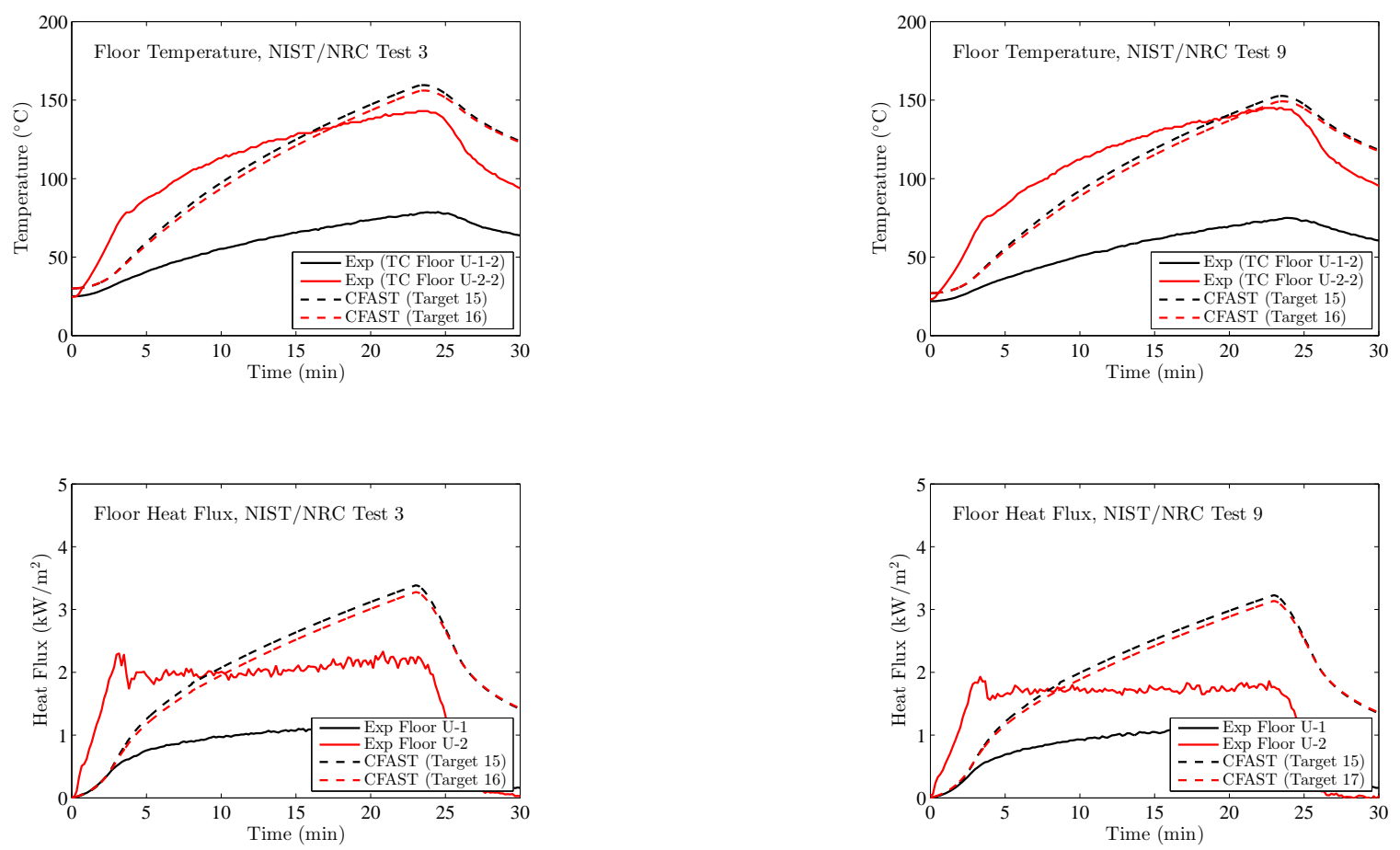

Figure B.71: NIST/NRC Series, Floor Temperature and Heat Flux, Replicate Tests 3 and 9.
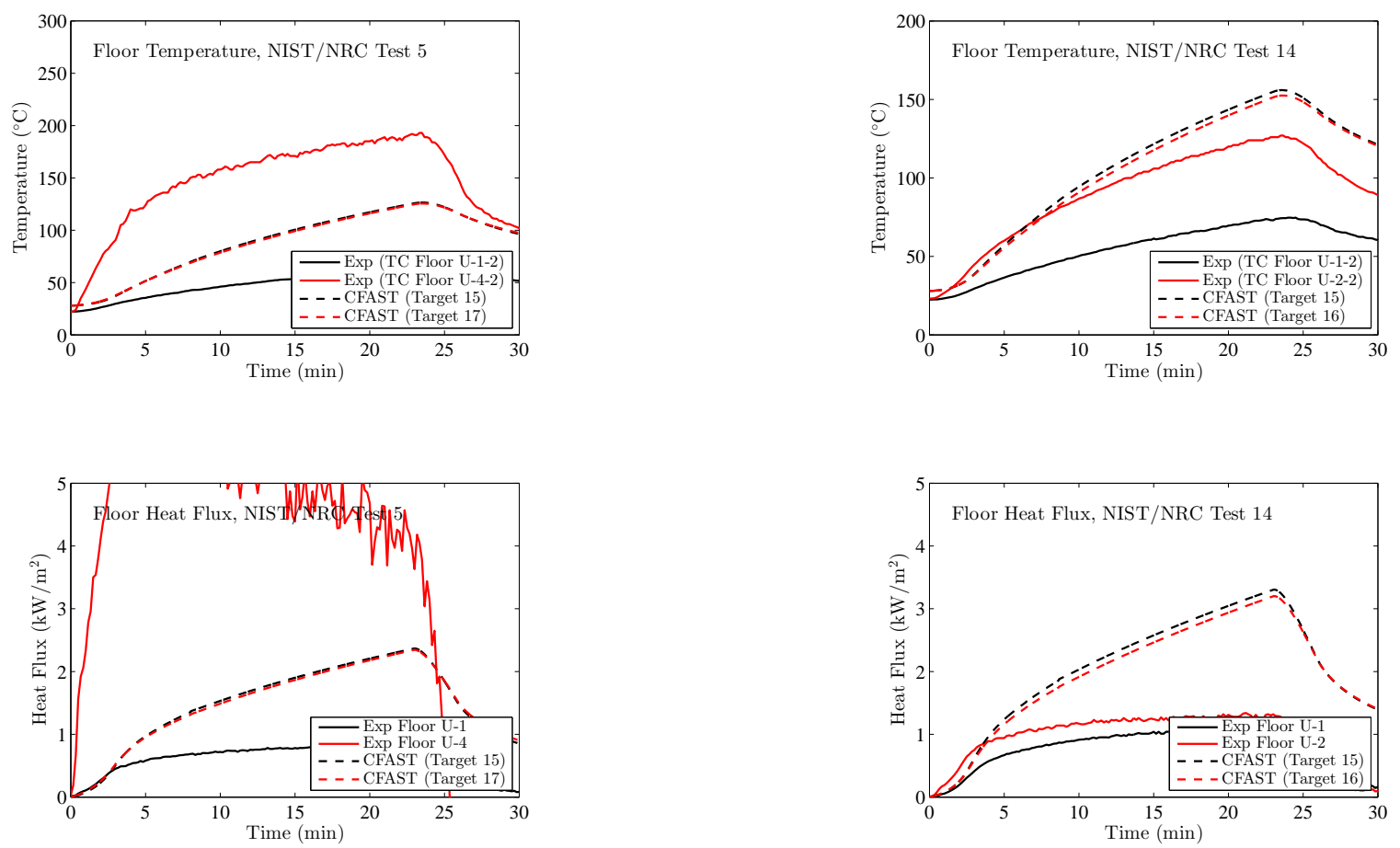

Figure B.72: NIST/NRC Series, Floor Temperature and Heat Flux, Replicate Tests 5 and 14. 

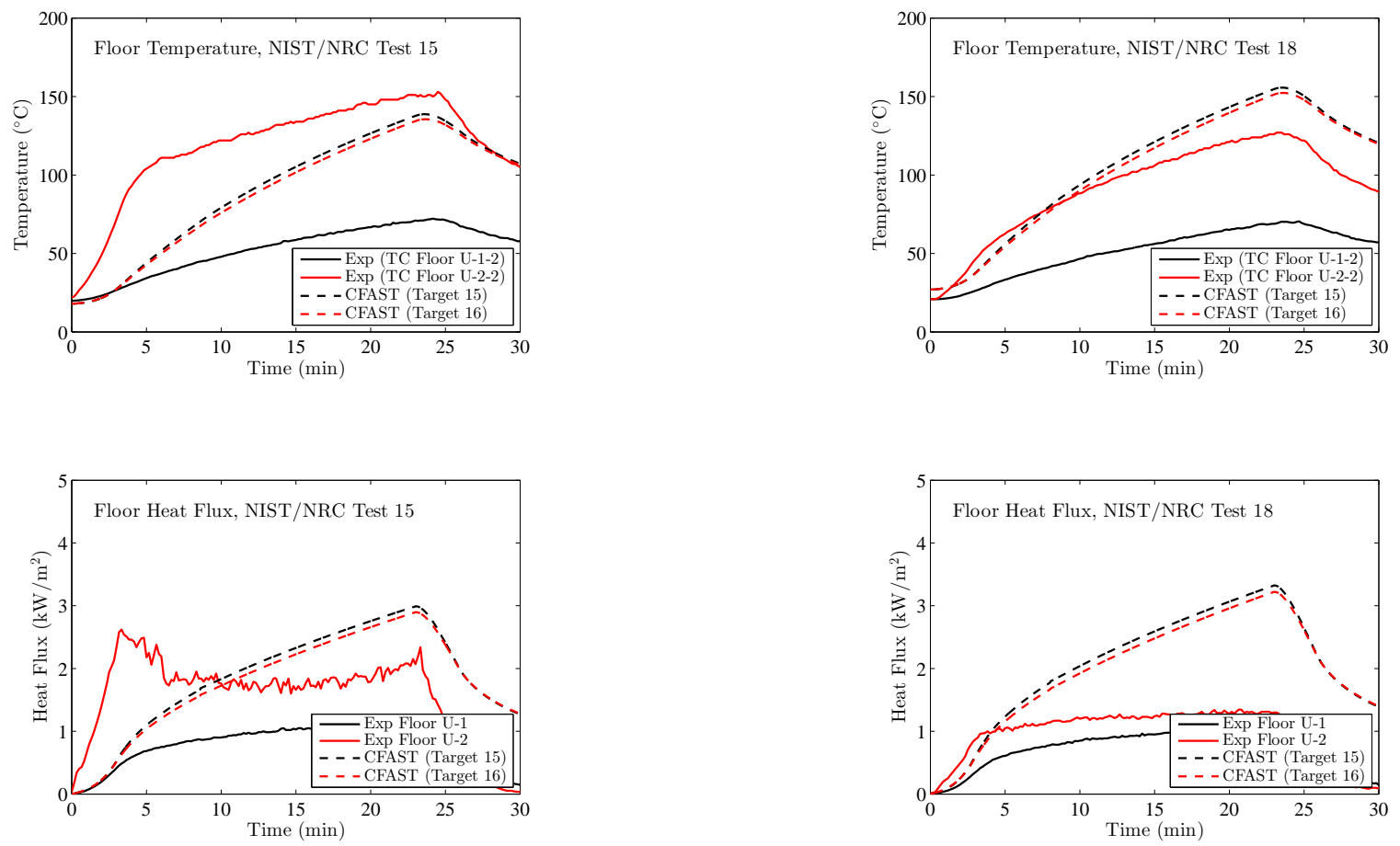

Figure B.73: NIST/NRC Series, Floor Temperature and Heat Flux, Replicate Tests 15 and 18. 


\section{B.4 FM/SNL Test Series}

The Factory Mutual and Sandia National Laboratories (FM/SNL) Test Series was a series of 25 fire tests conducted in 1985 for the NRC by Factory Mutual Research Corporation (FMRC), under the direction of Sandia National Laboratories (SNL). The primary purpose of these tests was to provide data with which to validate computer models for various types of NPP compartments. The experiments were conducted in an enclosure measuring $18 \mathrm{~m} \times 12 \mathrm{~m} \times 6 \mathrm{~m}$, constructed at the FMRC fire test facility in Rhode Island. The FM/SNL test series is described in detail, including the types and locations of measurement devices, as well as some results in References [64, 65]. 

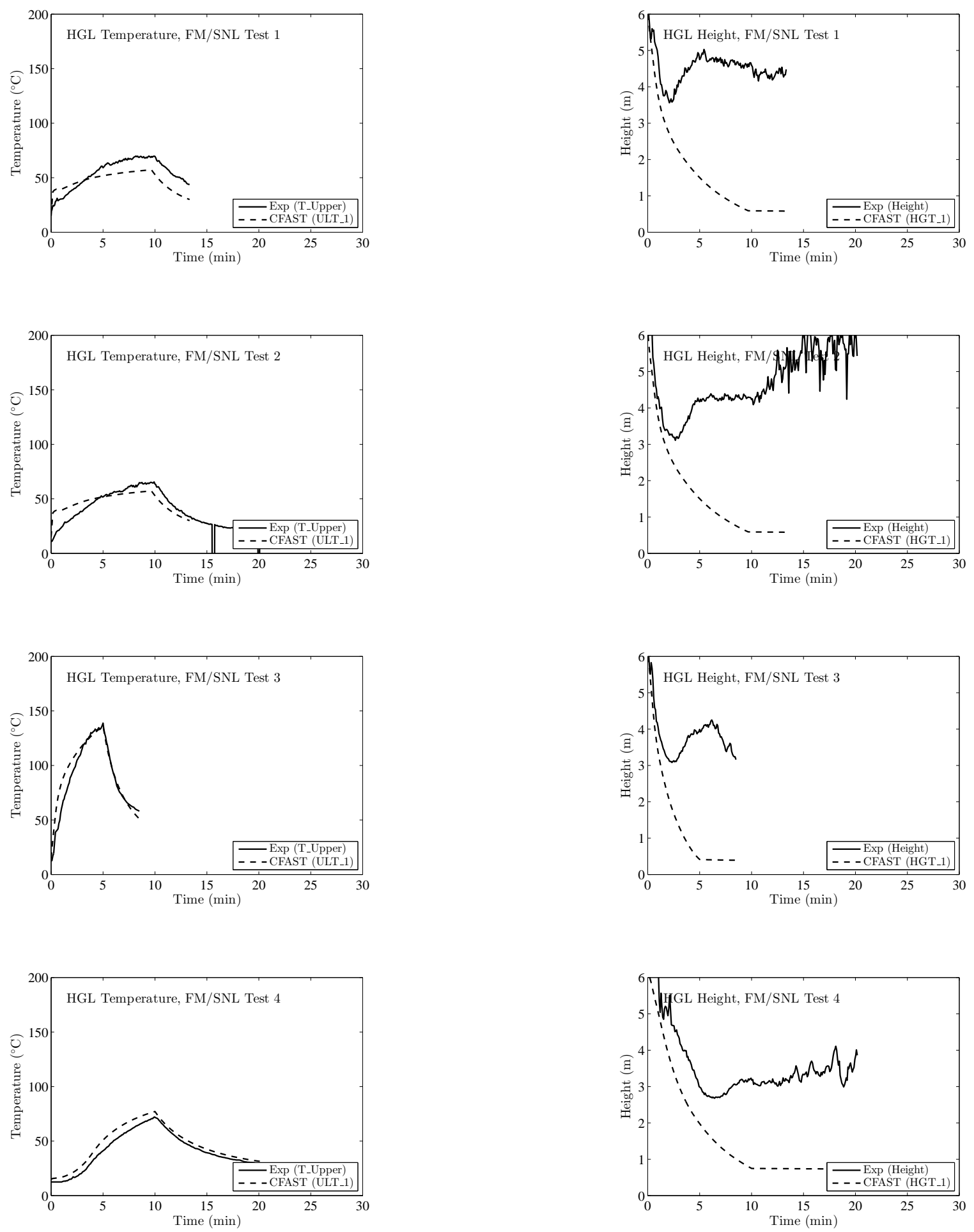

Figure B.74: Hot Gas Layer Temperature and Height for the FM/SNL Tests. 

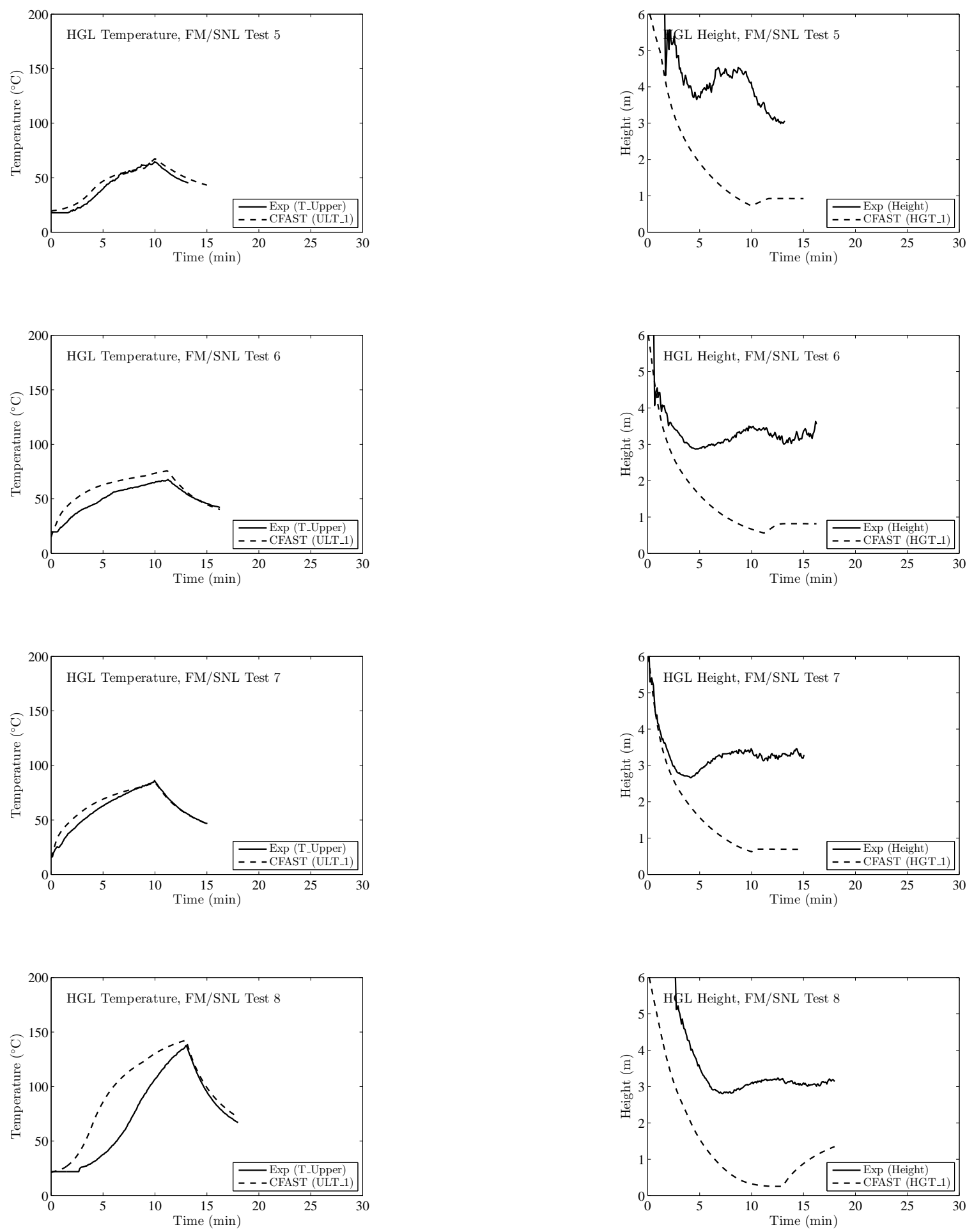

Figure B.75: Hot Gas Layer Temperature and Height for the FM/SNL Tests. 

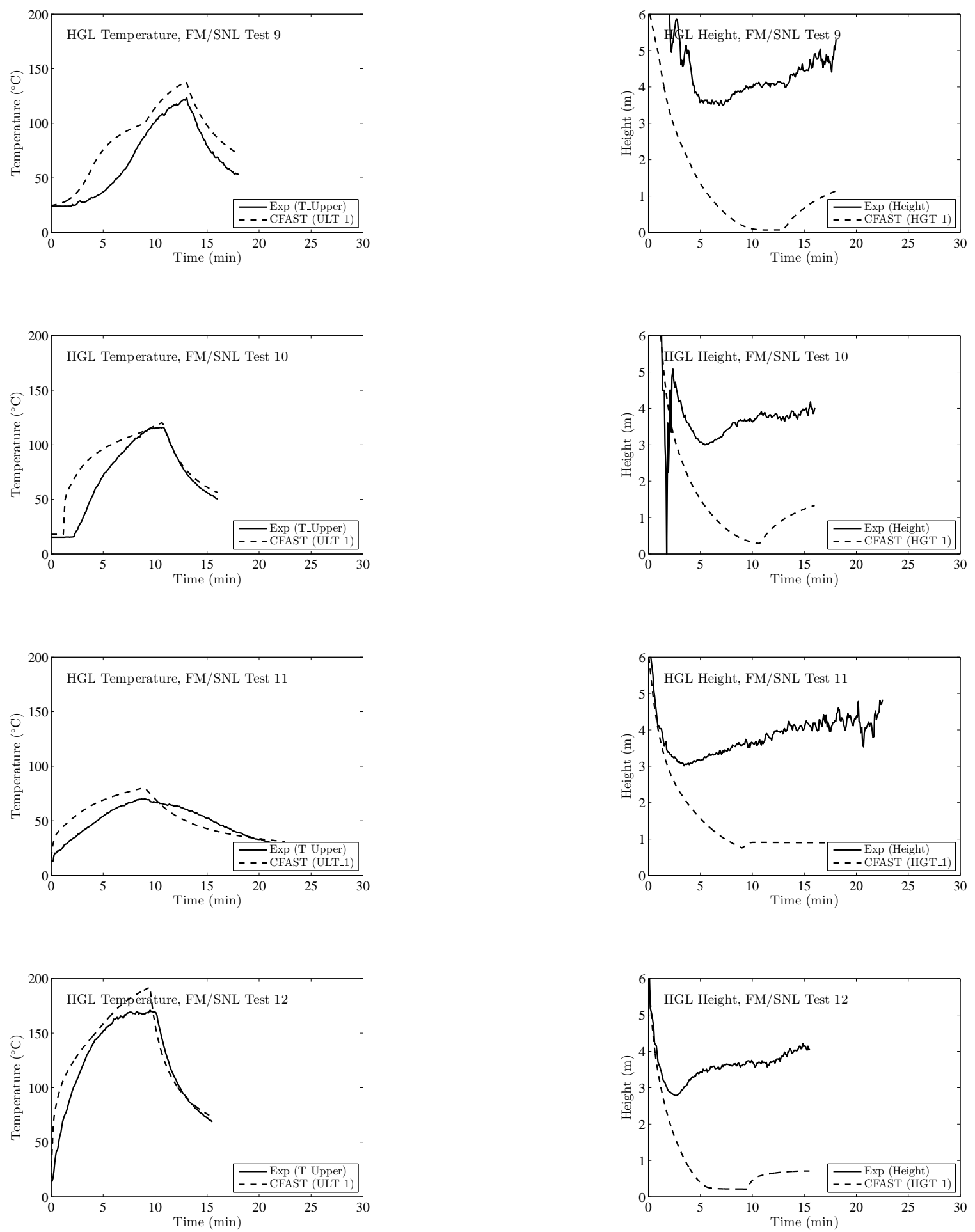

Figure B.76: Hot Gas Layer Temperature and Height for the FM/SNL Tests. 

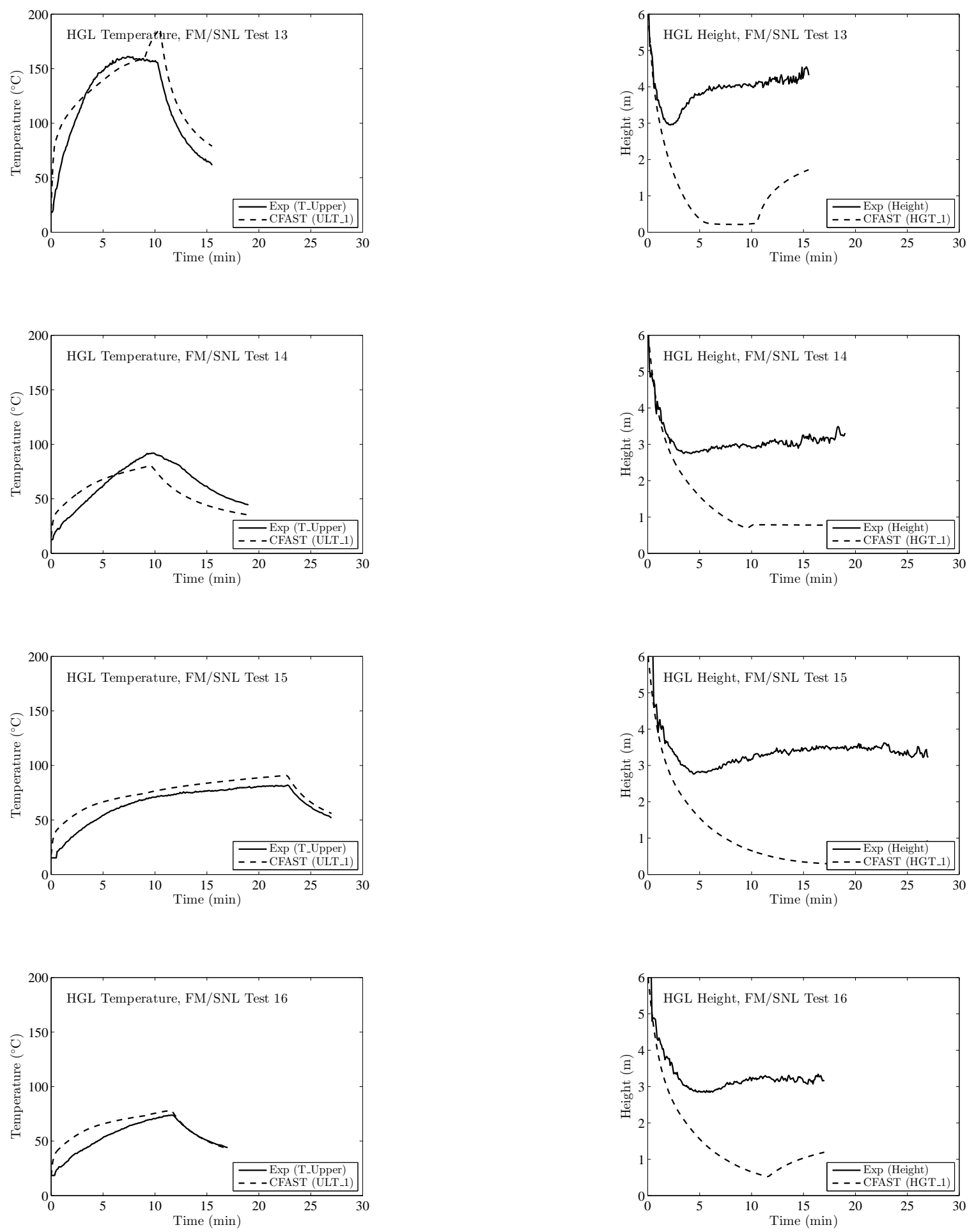

Figure B.77: Hot Gas Layer Temperature and Height for the FM/SNL Tests. 

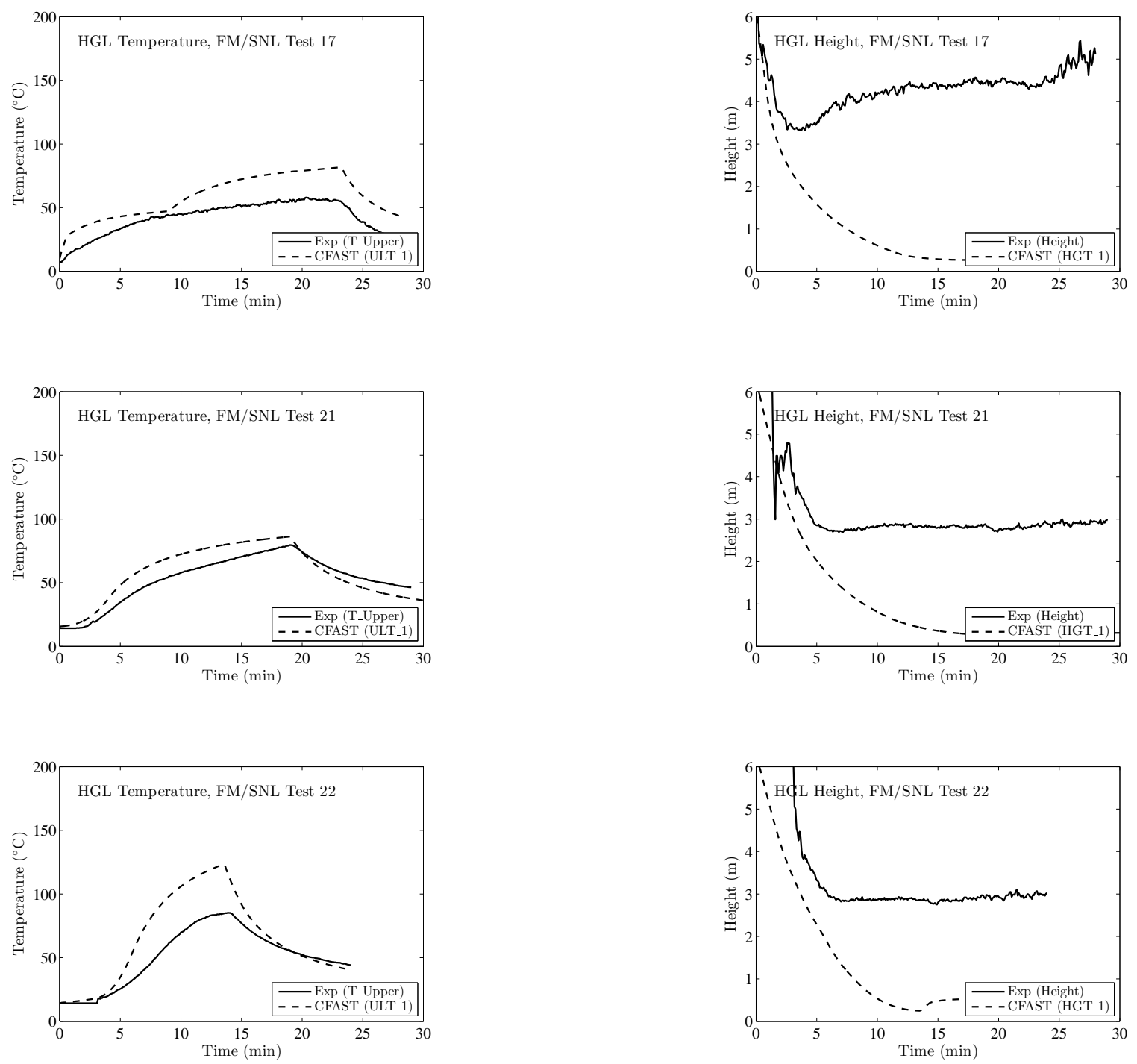

Figure B.78: Hot Gas Layer Temperature and Height for the FM/SNL Tests. 

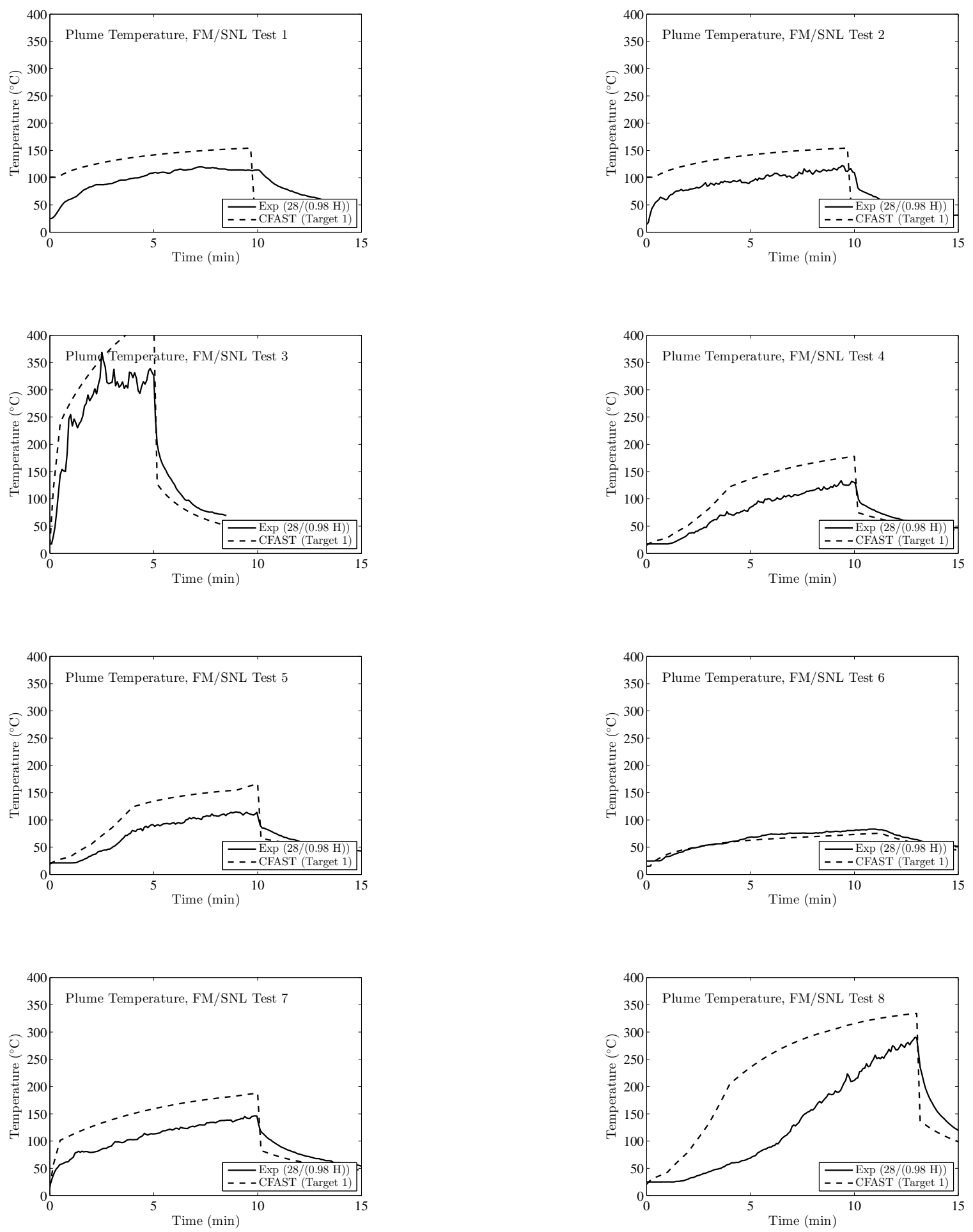

Figure B.79: Predicted Plume Centerline Temperature for the FM/SNL Tests. 

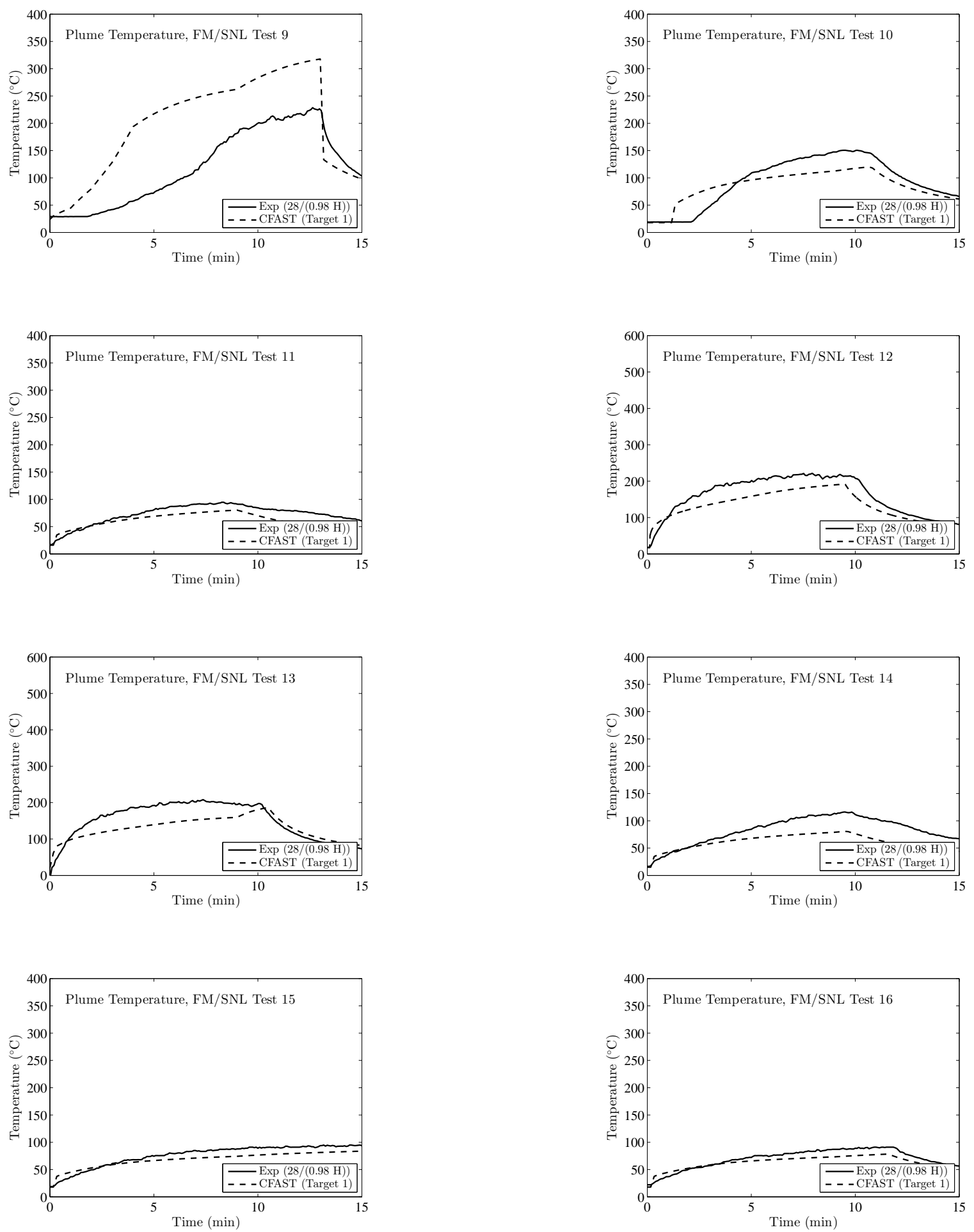

Figure B.80: Predicted Plume Centerline Temperature for the FM/SNL Tests. 

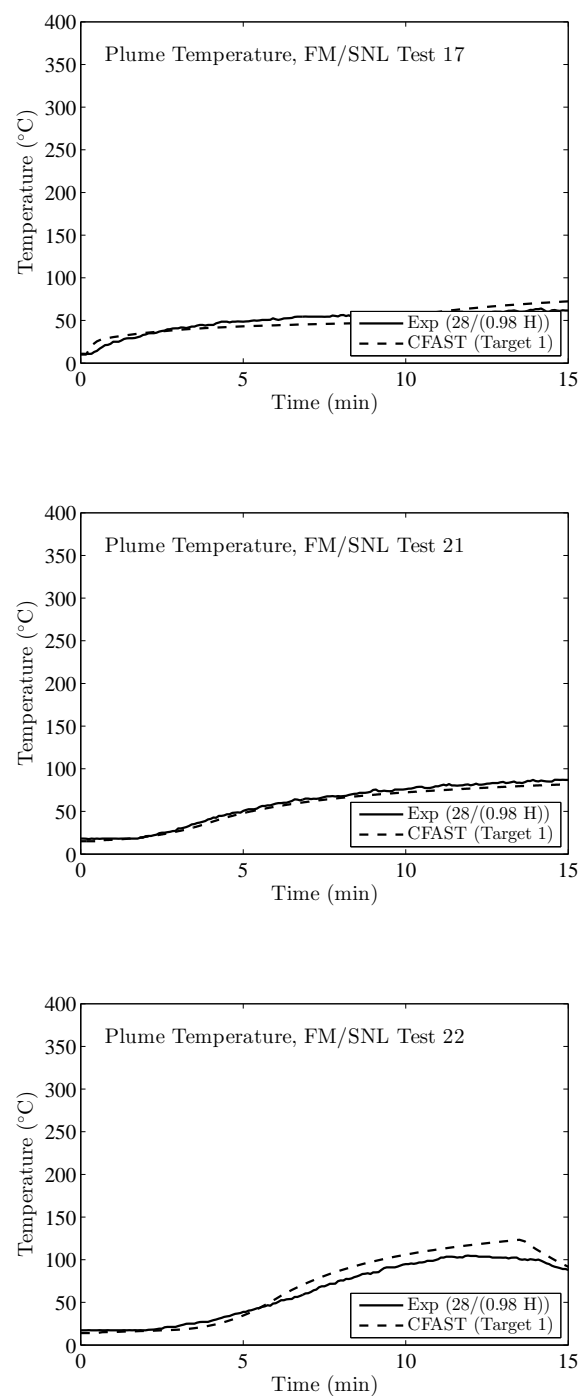

Figure B.81: Predicted Plume Centerline Temperature for the FM/SNL Tests. 

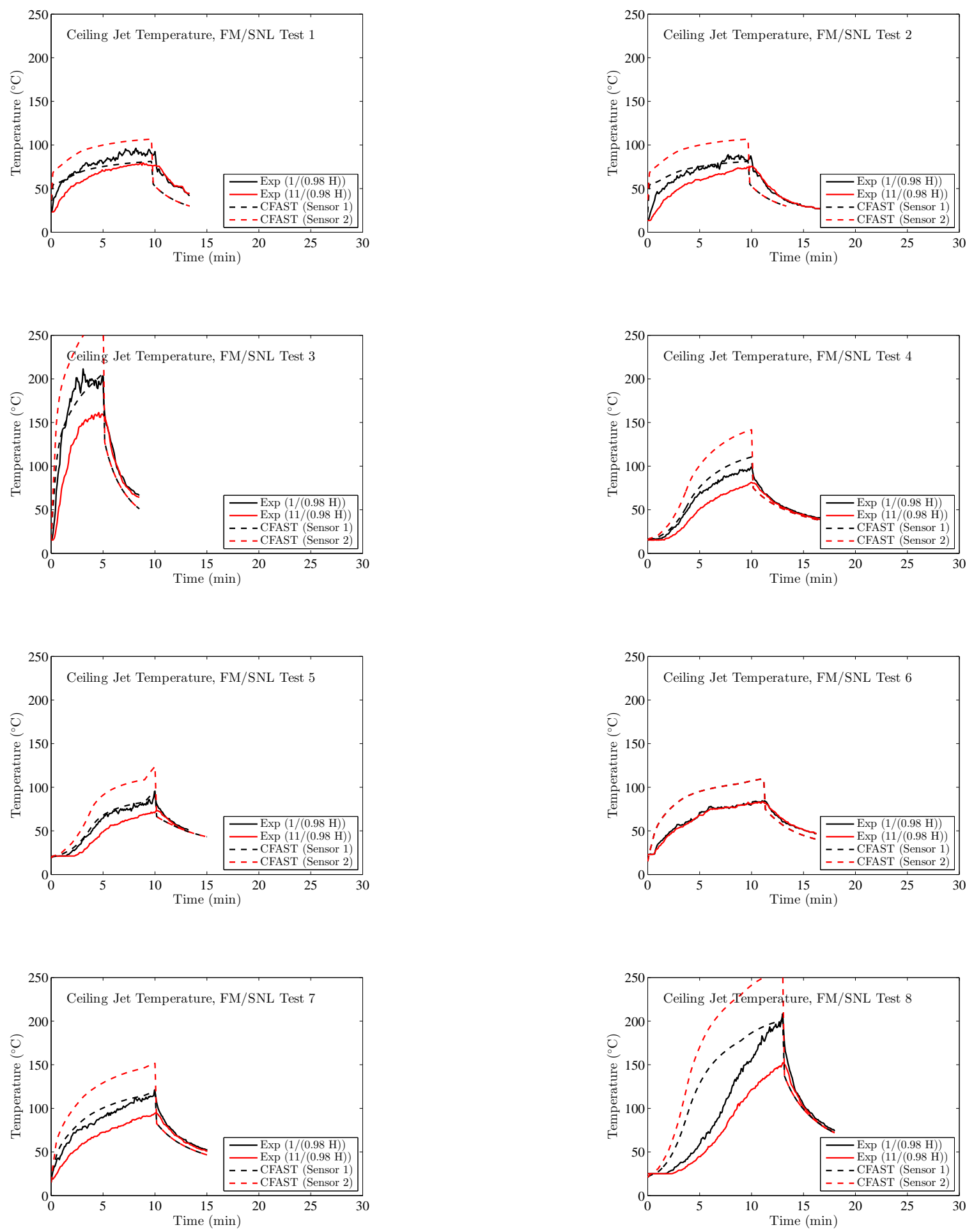

Figure B.82: Predicted Plume Centerline Temperature for the FM/SNL Tests. 

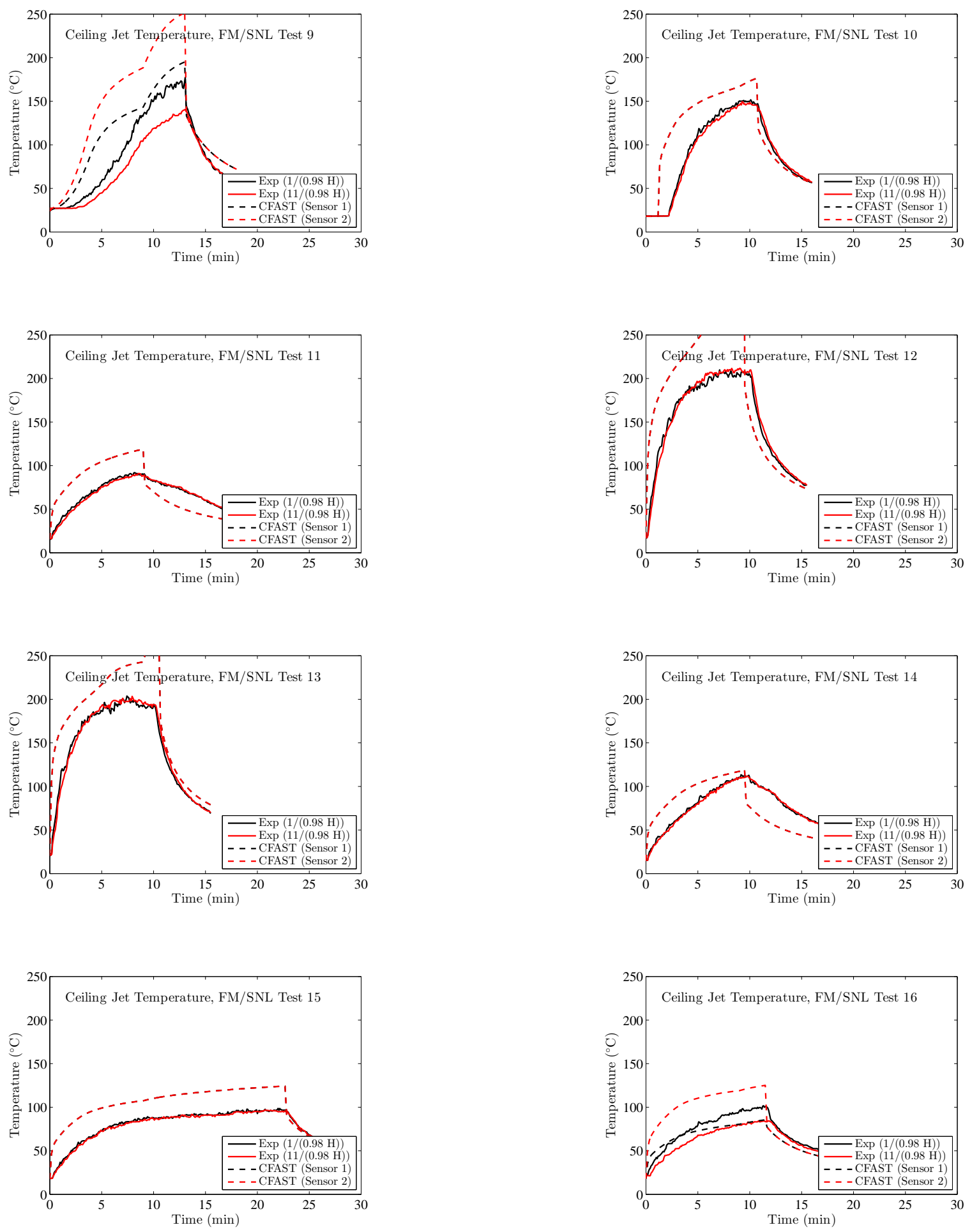

Figure B.83: Predicted Plume Centerline Temperature for the FM/SNL Tests. 

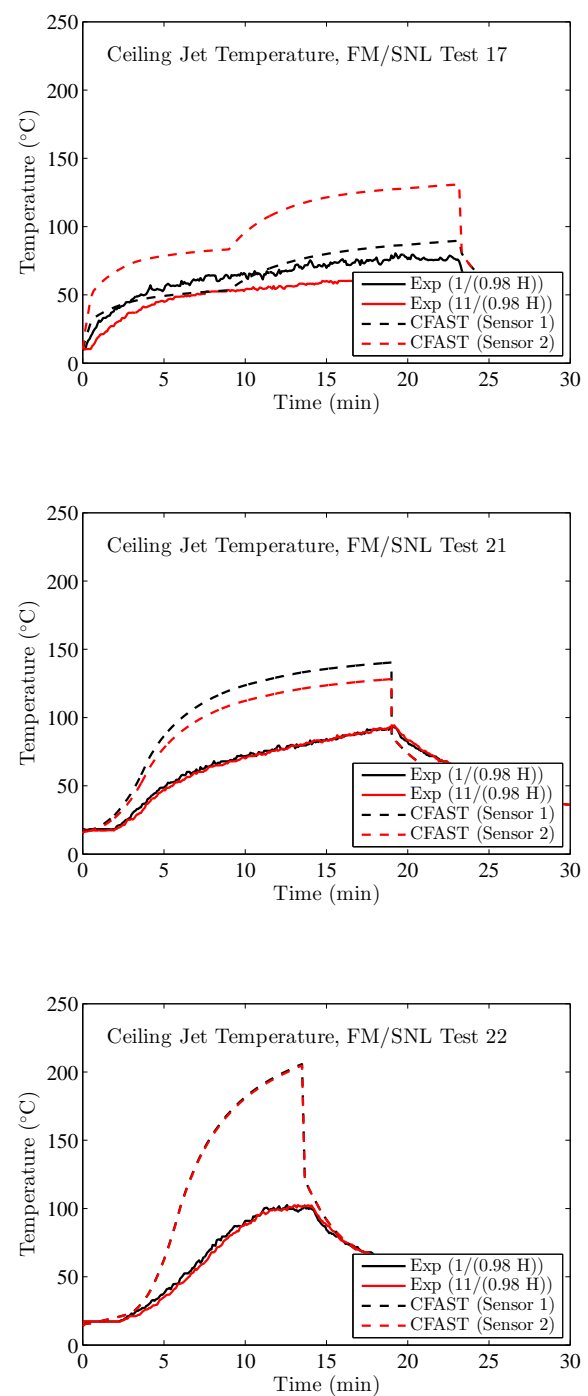

Figure B.84: Predicted Plume Centerline Temperature for the FM/SNL Tests. 


\section{B.5 iBMB Compartment Tests}

A series of small compartment kerosene pool fire experiments, conducted at the Institut fr Baustoffe, Massivbau und Brandschutz (iBMB) of Braunschweig University of Technology in Germany in 2004 [66]. The results from Test 1 were considered here. These experiments involved relatively large fires in a relatively small $(3.6 \mathrm{~m}$ x $3.6 \mathrm{~m}$ x $5.7 \mathrm{~m})$ concrete enclosure.

A second series of fire experiments in 2004, conducted under the International Collaborative Fire Model Project (ICFMP) involved realistically routed cable trays inside the same concrete enclosure at iBMB [67]. The compartment was configured slightly differently with a ceiling height of $5.6 \mathrm{~m}$. Six types of measurements conducted during the test series were used in the evaluation conducted here, including the HGL temperature and depth, oxygen gas concentration, the temperature of targets and compartment surfaces, and heat flux. 

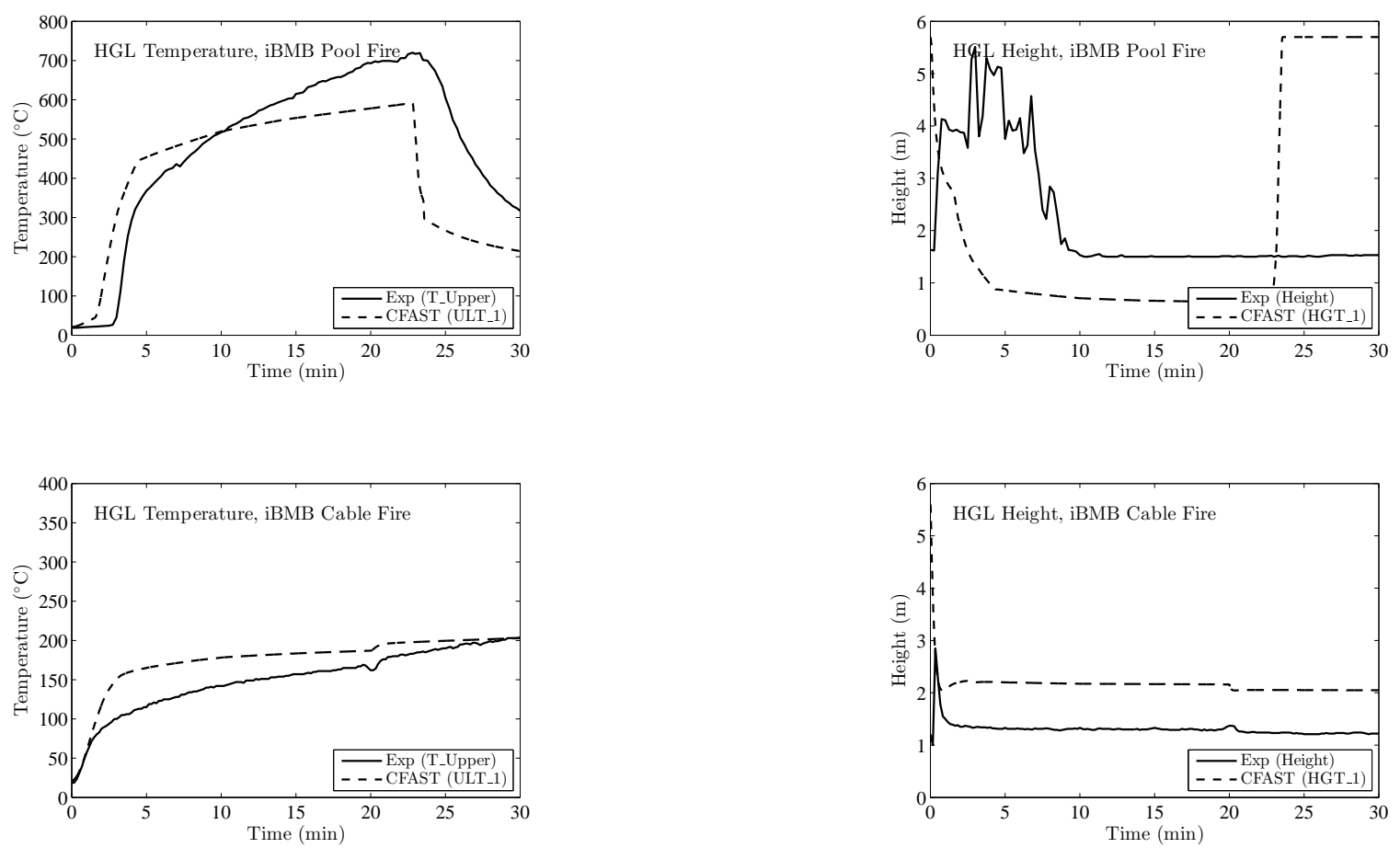

Figure B.85: Predicted HGL Temperature and Height for the iBMB Single Compartment Tests.
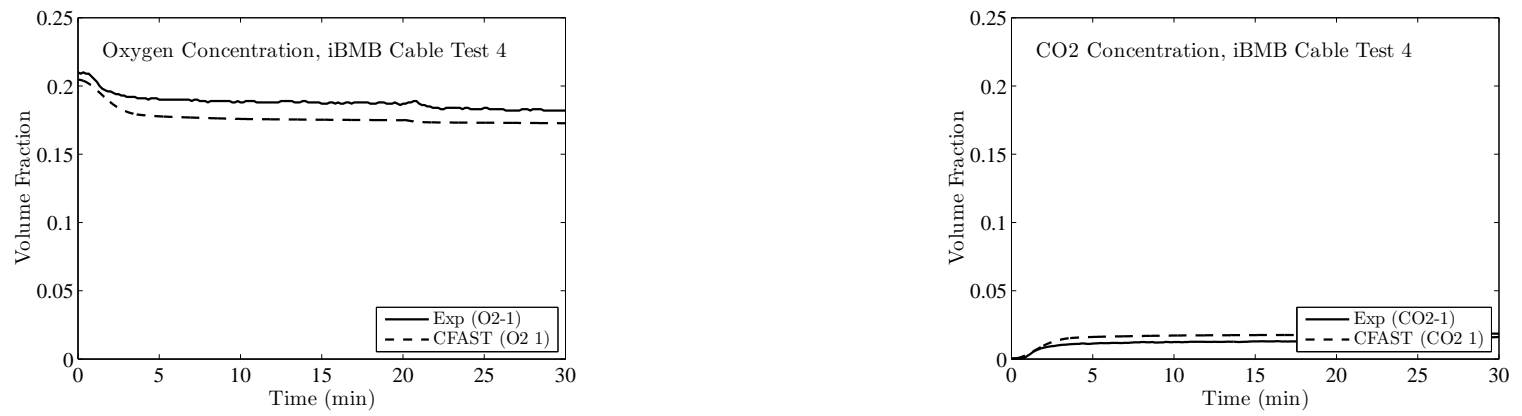

Figure B.86: Predicted Oxygen and Carbon Dioxide for the iBMB Cable Test 5. 


\section{B.6 NBS Multi-Compartment Test Series}

The National Bureau of Standards (NBS, former name of NIST) Multi-Compartment Test Series consisted of 45 fire tests representing 9 different sets of conditions were conducted in a three-room suite. The experiments were conducted in 1985 and are described in detail in reference [68]. The suite consisted of two relatively small rooms, connected via a relatively long corridor. Total volume of the structure was approximately $100 \mathrm{~m}^{2}$. The fire source, a gas burner, was located against the rear wall of one of the small compartments. Fire tests of $100 \mathrm{~kW}, 300 \mathrm{~kW}$ and $500 \mathrm{~kW}$ were conducted. For the current study, three $100 \mathrm{~kW}$ fire experiments have been used, including Test 100A from Set 1, Test 1000 from Set 2, and Test 100Z from Set 4. For the NBS Multi-room series, Tests 100A, $100 \mathrm{O}$ and 100Z were selected for study, because they were constructively used in a previous validation study [[69], and because these tests had the steadiest values of measured heat

release rate during the steady burning period. The selected data are also available in Reference [69]. 

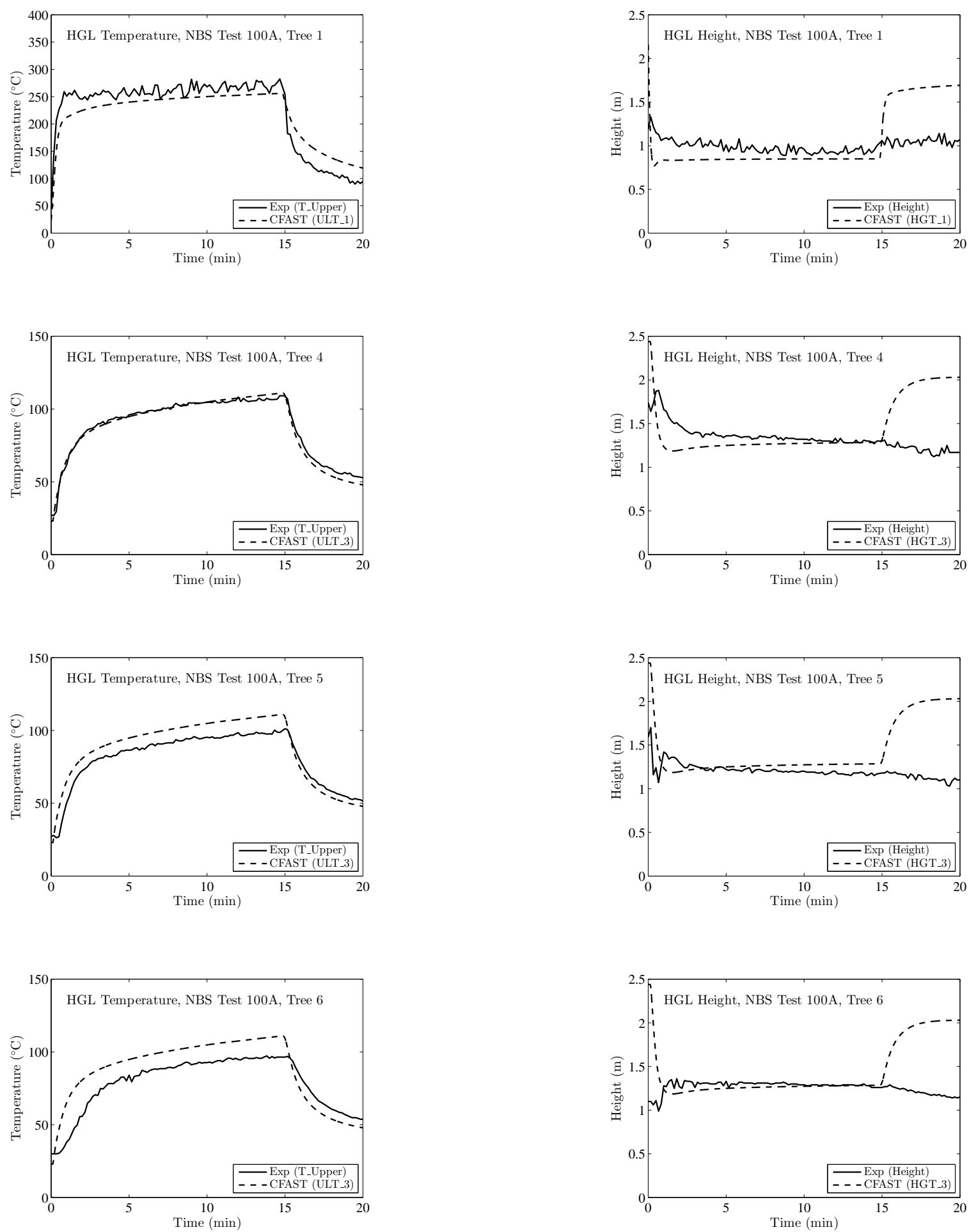

Figure B.87: Hot Gas Layer Temperature and Height for the NBS Multi-Room Test 100A. 

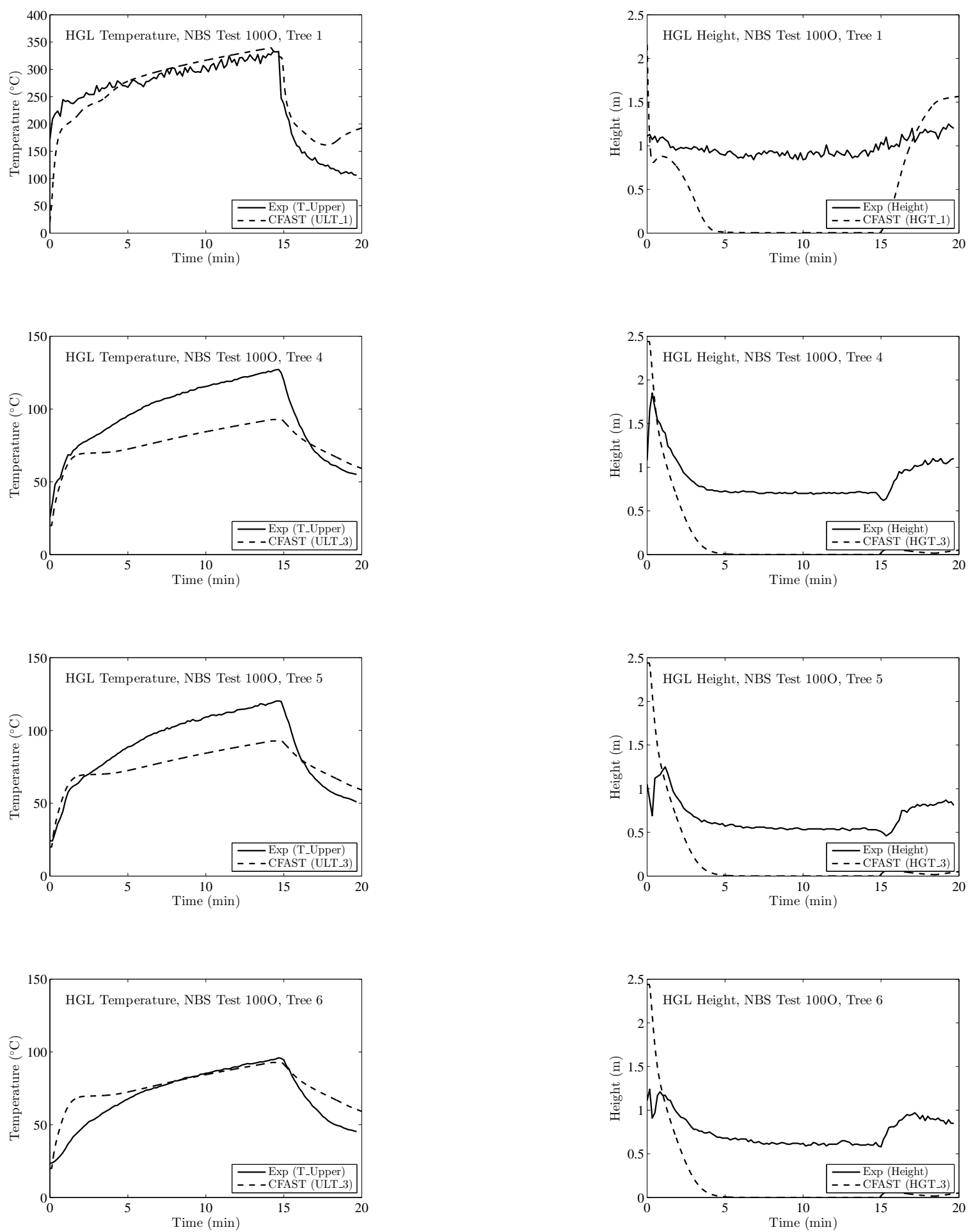

Figure B.88: Hot Gas Layer Temperature and Height for the NBS Multi-Room Test 1000. 

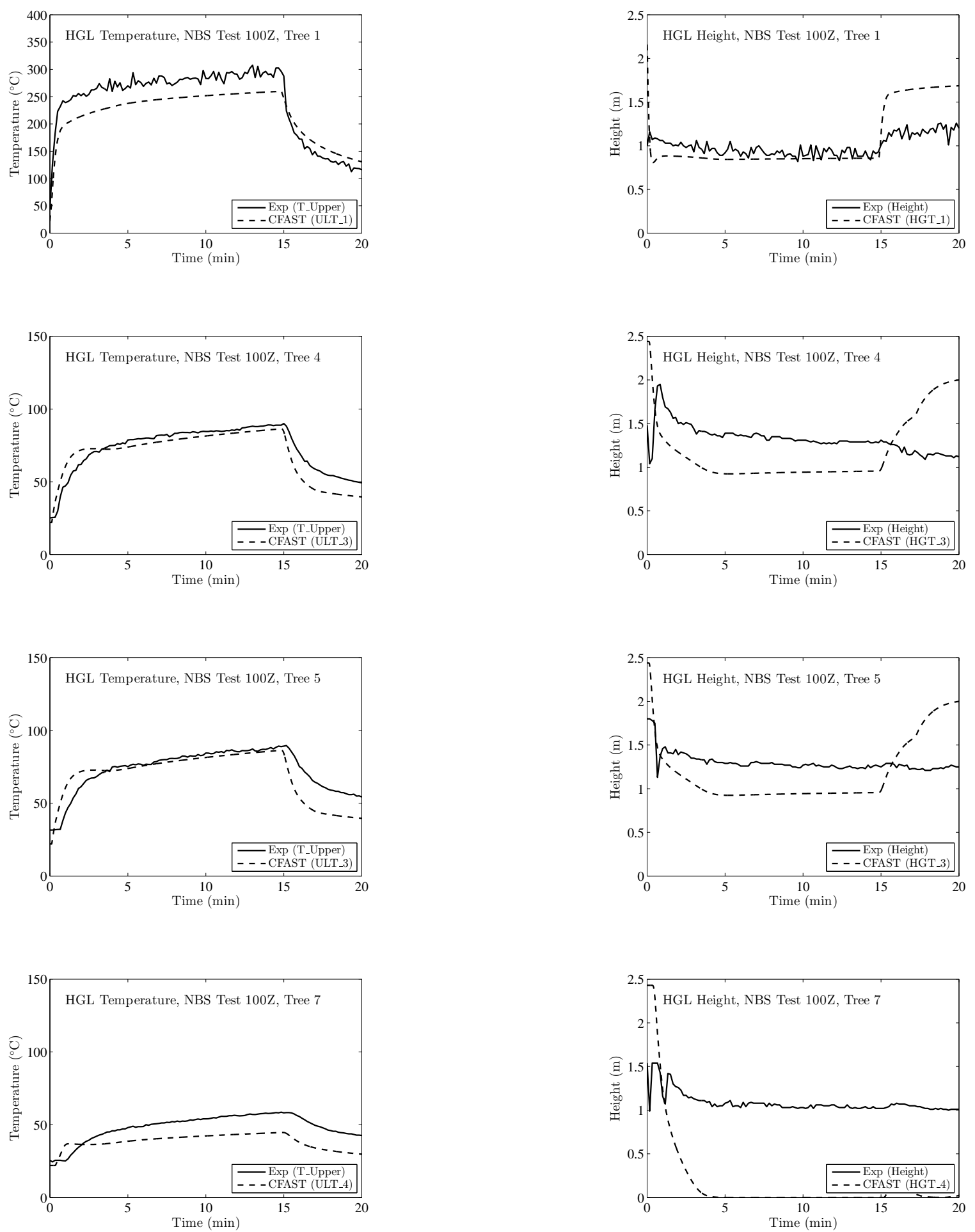

Figure B.89: Hot Gas Layer Temperature and Height for the NBS Multi-Room Test 100Z. 


\section{B.7 FM Four Room Including Corridor Test Series}

This data set describes a series of tests conducted in a multiple room configuration with more complex gas burner fires than the previous data set. This study [70] was included because, in many ways, it is similar to the smoke movement study performed at NBS [68], and permits comparisons between two different laboratories. In addition, it expands upon that data set by providing larger a time-varying gas burner fires in a room-corridor configuration. Fire size was about up to $1 \mathrm{MW}$ with a total volume of $200 \mathrm{~m}^{3}$. This study was performed to collect data allowing for variations in fire source, ventilation, and geometry in a multi-compartment structure, especially for situations with closed doors. 

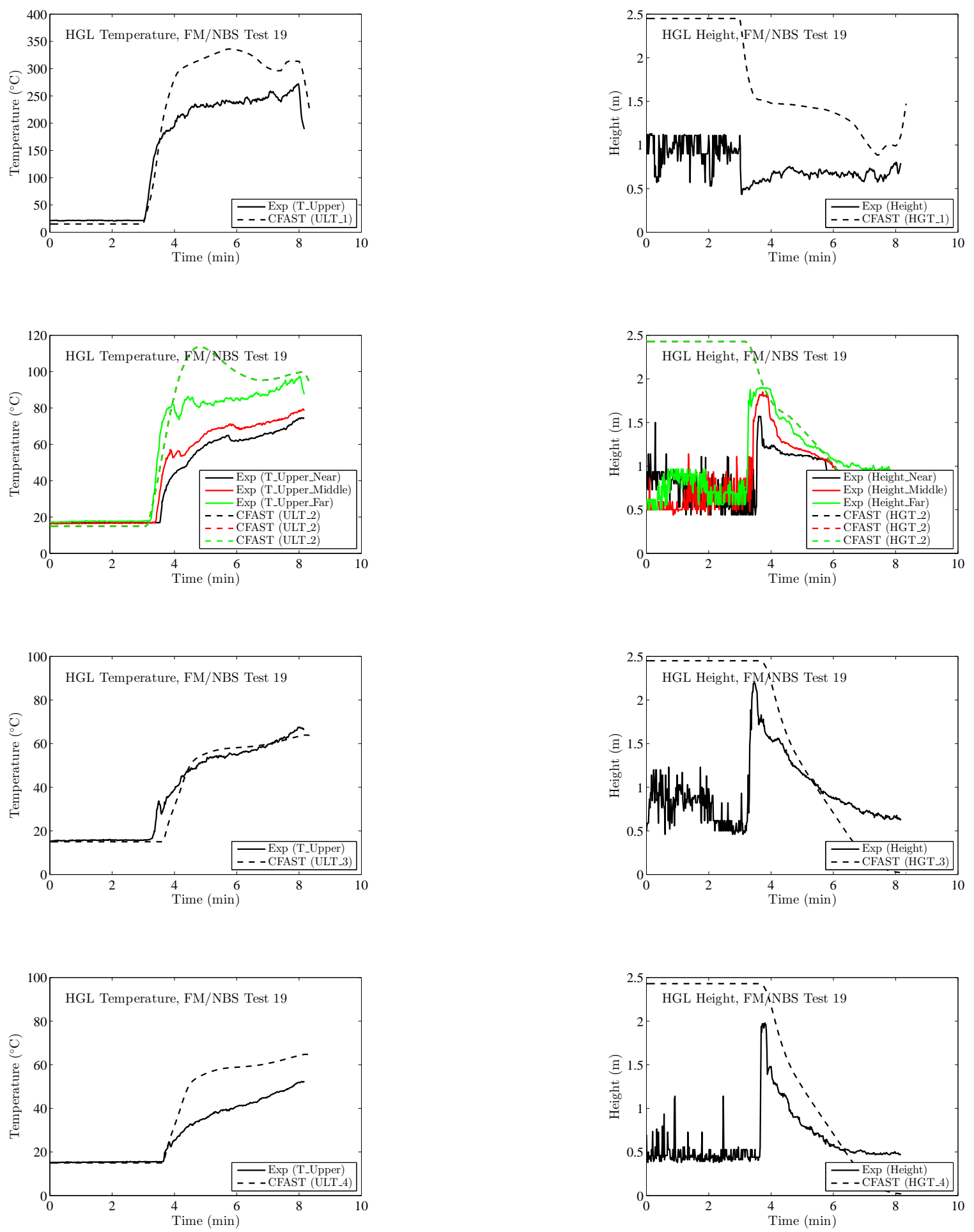

Figure B.90: Hot Gas Layer Temperature and Height for the FM/NBS Four Compartment Test 19. 

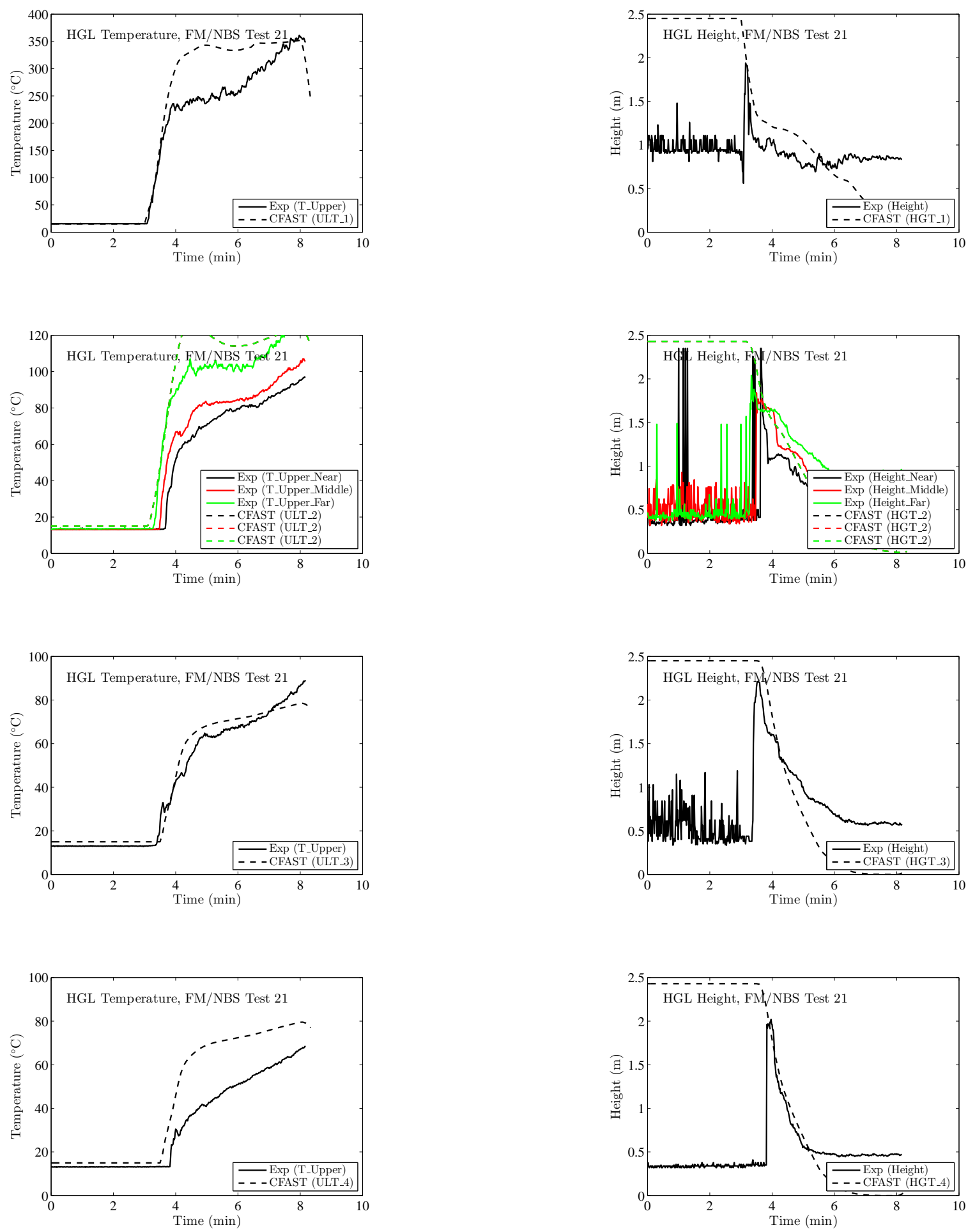

Figure B.91: Hot Gas Layer Temperature and Height for the FM/NBS Four Compartment Test 21. 

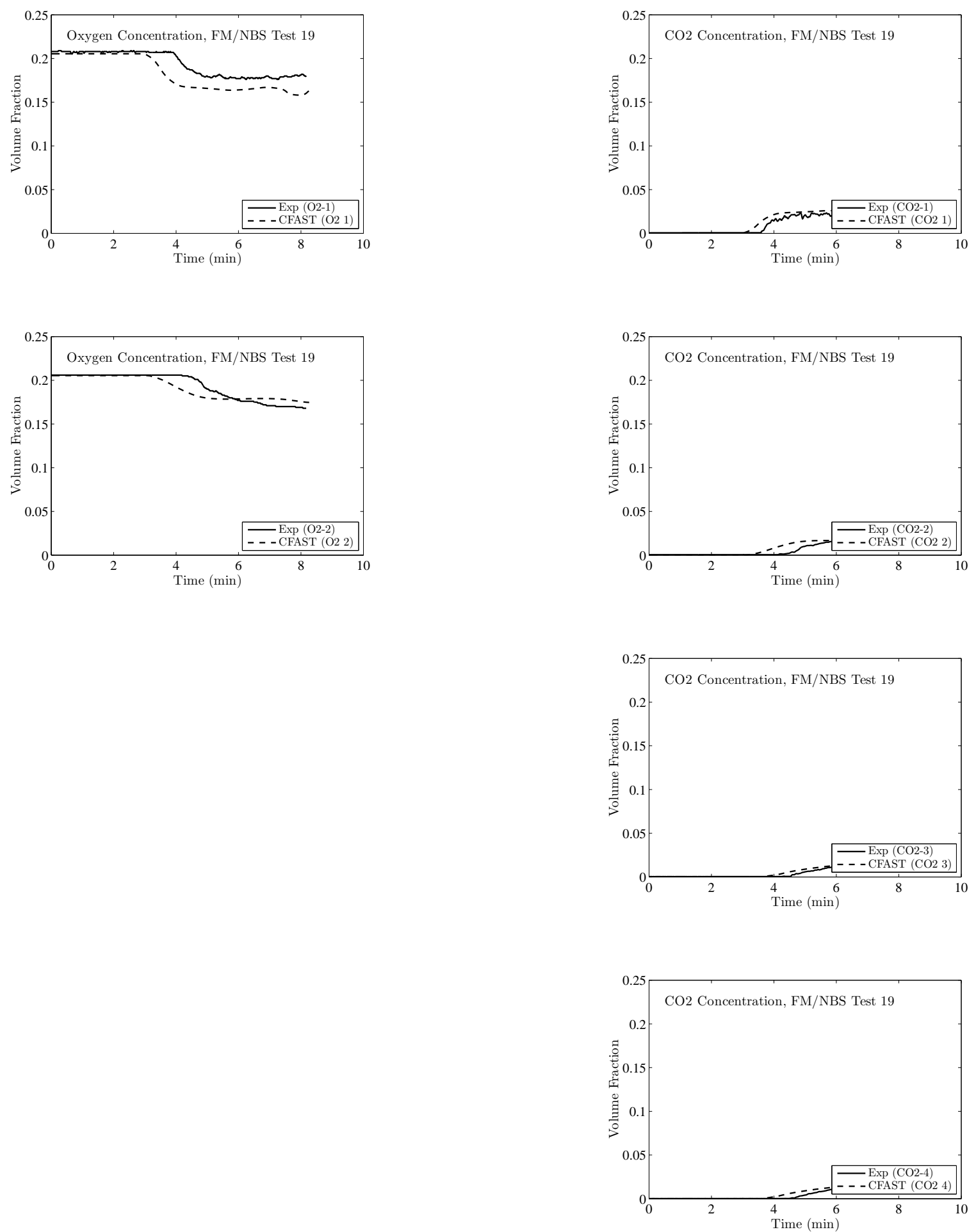

Figure B.92: Oxygen and Carbon Dioxide Concentration for the FM/NBS Test 19. 

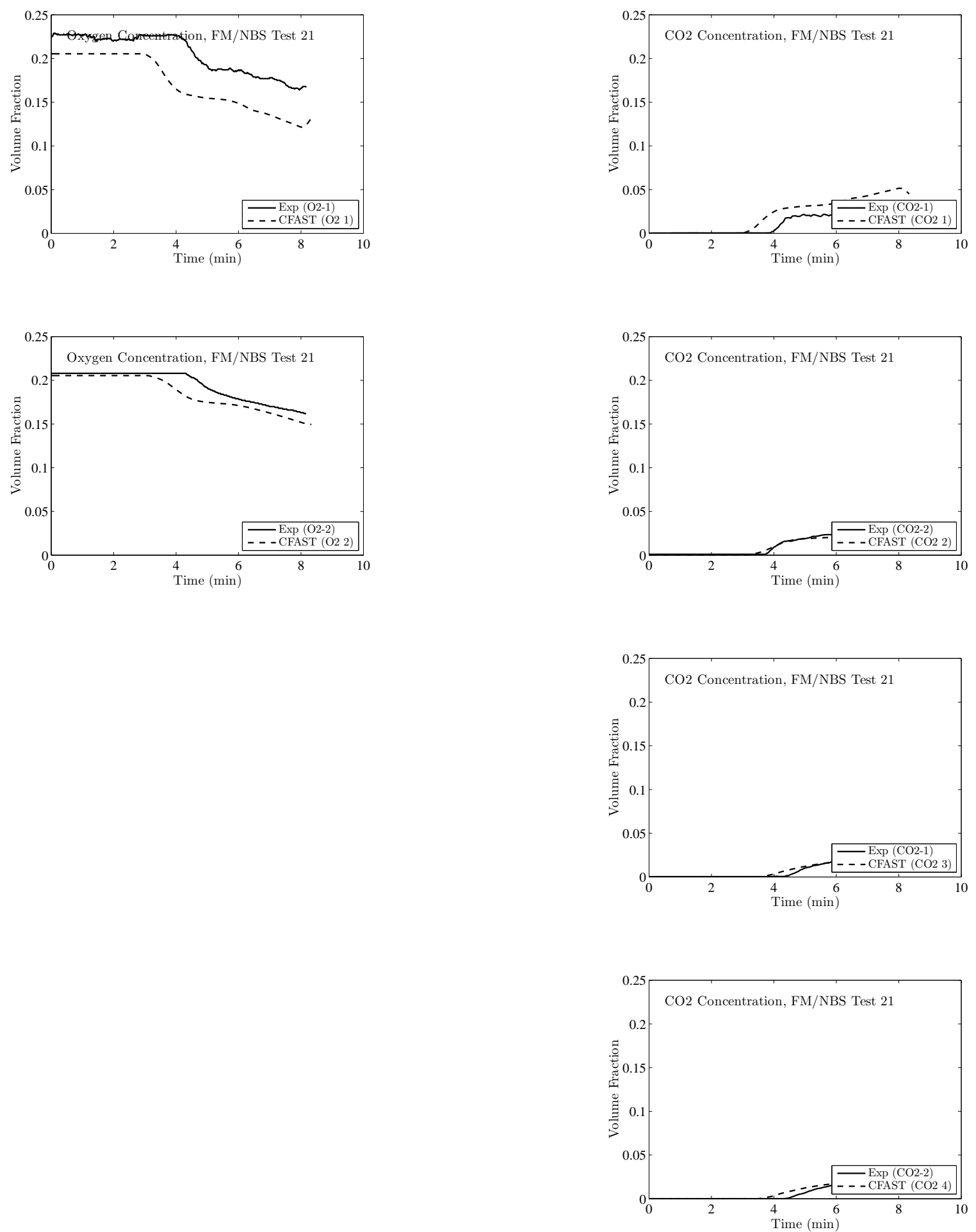

Figure B.93: Oxygen and Carbon Dioxide Concentration for the FM/NBS Test 21. 


\section{B.8 NIST Seven-story Hotel Tests}

By far the most complex test, this data set is part of a series of full-scale experiments conducted to evaluate zoned smoke control systems, with and without stairwell pressurization [71]. It was conducted in a seven story hotel with multiple rooms on each floor and a stairwell connecting all floors. This data set was chosen because it would challenge the scope of most current fire models. Measured temperatures and pressure differences between the rooms and floors of the building are extensive and consistent. Peak fire size was $3 \mathrm{MW}$ with a total building volume of $140000 \mathrm{~m}^{3}$. The hotel was a masonry structure consisting of two wings, one three stories and the other seven stories tall. The fires were set on the second floor of the seven-story wing. 

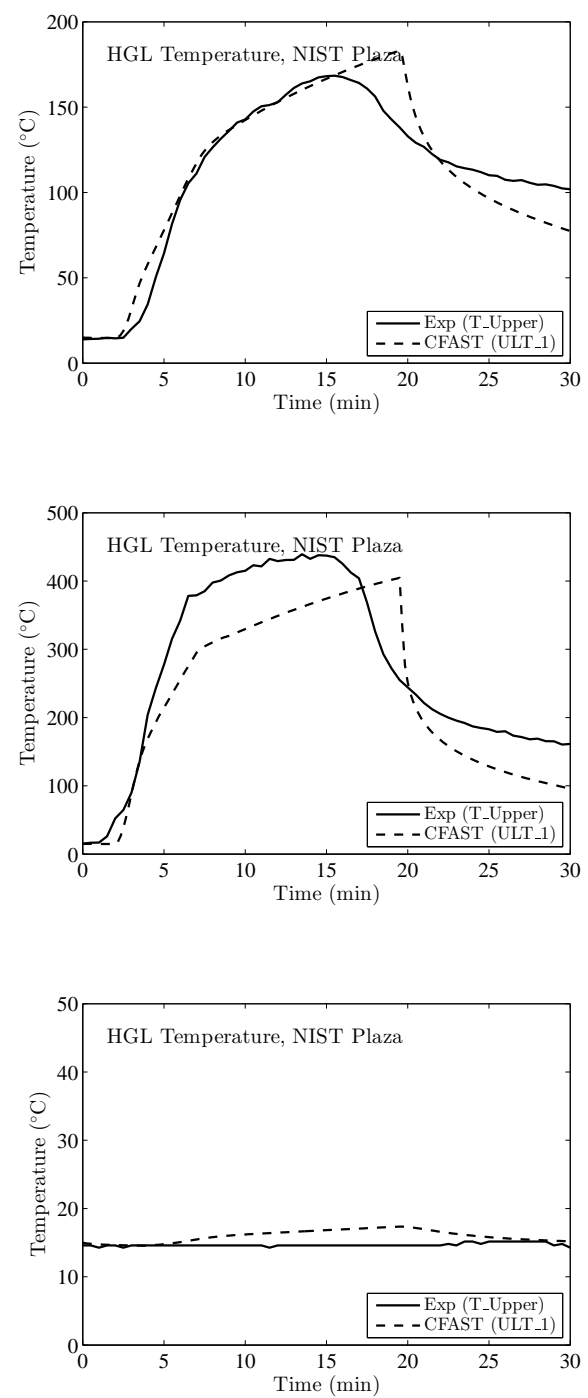

Figure B.94: Predicted HGL Temperature and Height for the Seven-Story Hotel Test 7. 

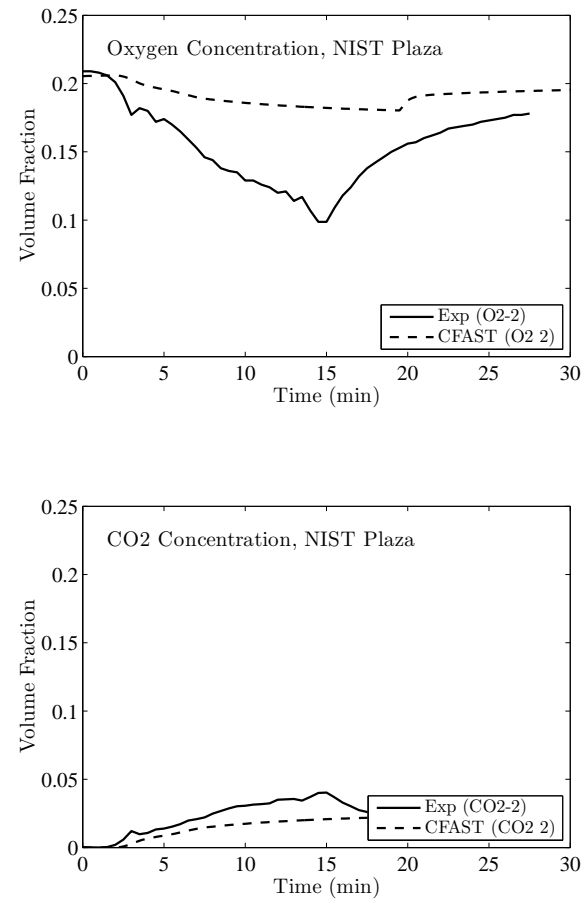

Figure B.95: Predicted Oxygen and Carbon Dioxide for the Seven-Story Hotel Test 7. 